Electronic Supporting Information

For

\title{
Trianionic Pincer Complexes of Niobium and Tantalum as Precatalysts for ROMP of Norbornene
}

Sudarsan VenkatRamani, Christopher D. Roland, James G. Zhang, Ion Ghiviriga, Khalil A.

\author{
Abboud, Adam S. Veige * \\ Department of Chemistry, Center for Catalysis, University of Florida \\ P.O. Box 117200, Gainesville, FL, 32611. \\ *Corresponding author: veige@,chem.ufl.edu,
}

Tel.: 352-392-9844; fax: 352-392-3255. 


\section{Table of Contents}

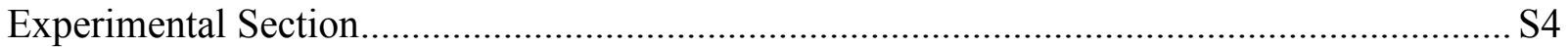

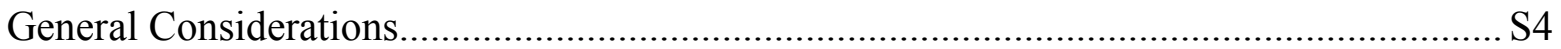

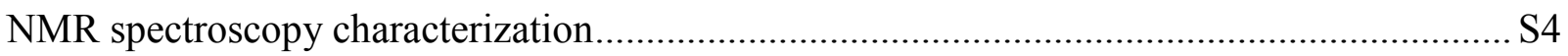

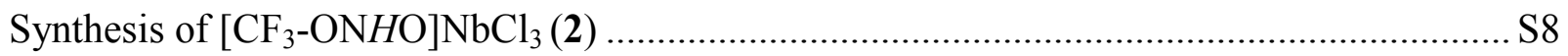

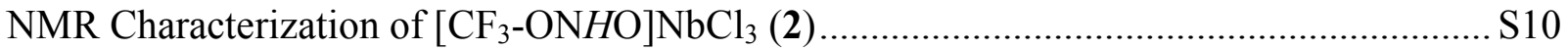

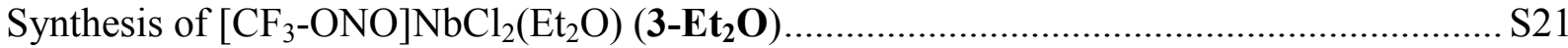

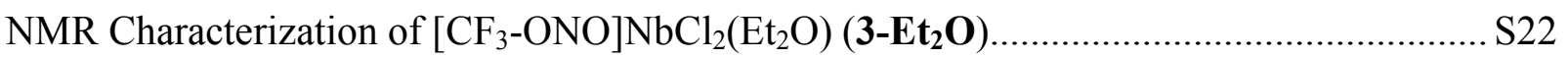

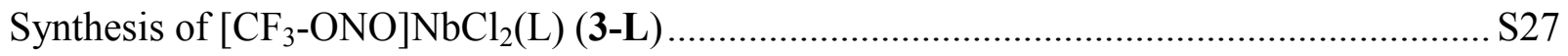

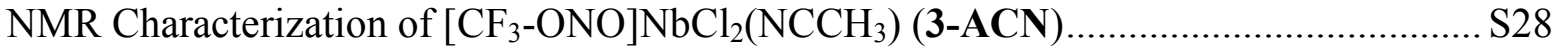

NMR Characterization of $\left[\mathrm{CF}_{3}-\mathrm{ONO}\right] \mathrm{NbCl}_{2}$ (py) (3-py) .............................................. S32

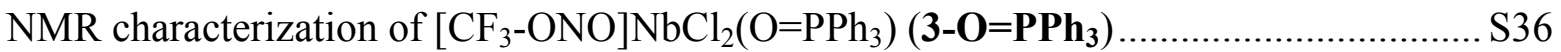

NMR characterization of $\left[\mathrm{CF}_{3}-\mathrm{ONO}\right] \mathrm{NbCl}_{2}$ (THF) (3-THF) .................................... S42

NMR Characterization of $\left[\mathrm{CF}_{3}-\mathrm{ONO}\right] \mathrm{NbCl}_{2}(\mathrm{DME})($ 3-DME) ....................................... S44

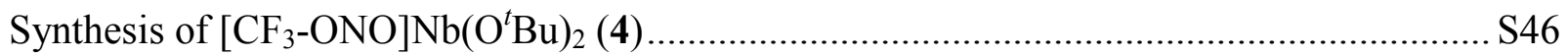

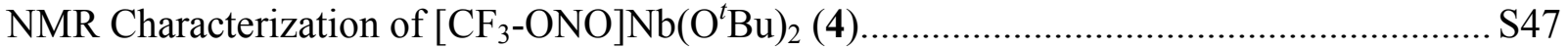

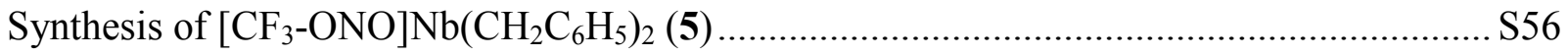

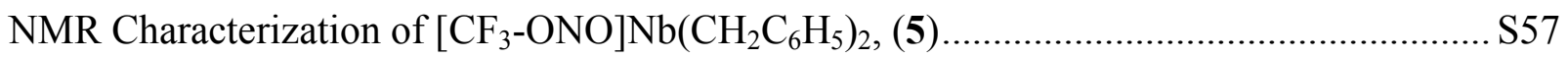

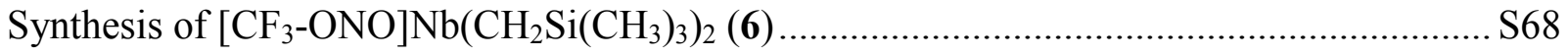


NMR Characterization of $\left[\mathrm{CF}_{3}-\mathrm{ONO}\right] \mathrm{Nb}\left(\mathrm{CH}_{2} \mathrm{Si}\left(\mathrm{CH}_{3}\right)_{3}\right)_{2}(\mathbf{6})$

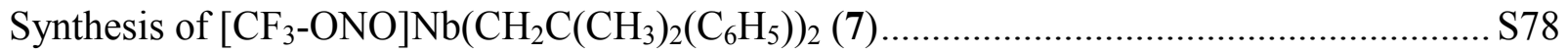

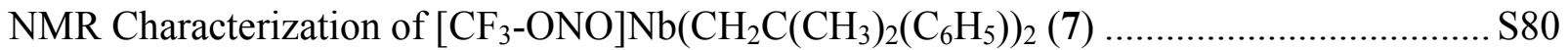

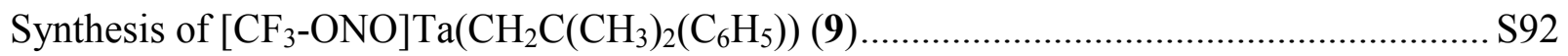

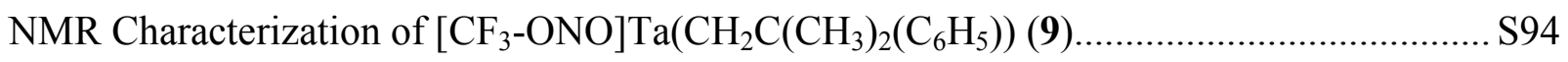

Polymerization of norbornene by $\left[\mathrm{CF}_{3}-\mathrm{ONO}\right] \mathrm{Nb}\left(\mathrm{CH}_{2} \mathrm{R}\right)_{2}$ (where $\mathrm{R}=\mathrm{C}_{6} \mathrm{H}_{5}(\mathbf{5}), \mathrm{Si}\left(\mathrm{CH}_{3}\right)_{3}(\mathbf{6})$,

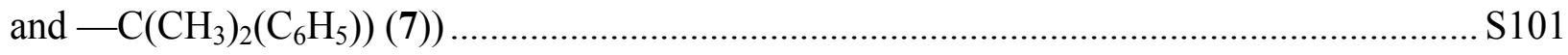

Polymerization of norbornene by $\left[\mathrm{CF}_{3}-\mathrm{ONO}\right] \mathrm{Nb}\left(\mathrm{CH}_{2} \mathrm{C}\left(\mathrm{CH}_{3}\right)_{2}\left(\mathrm{C}_{6} \mathrm{H}_{5}\right)\right)(7) \ldots \ldots \ldots \ldots \ldots \ldots . . . . \mathrm{S} 101$

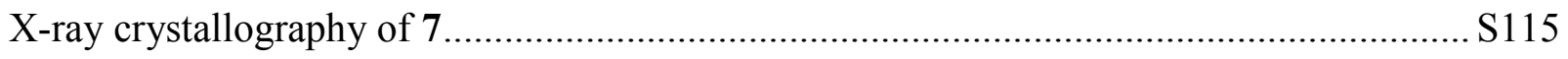

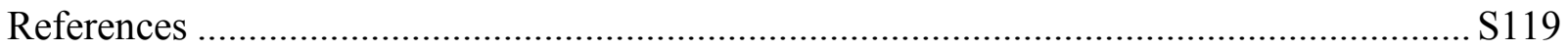




\section{Experimental Section}

\section{General Considerations}

Unless specified otherwise, all manipulations were performed under an inert atmosphere using standard Schlenk or glove box techniques. Glassware was oven-dried before use. Pentane, hexane, toluene, diethyl ether $\left(\mathrm{Et}_{2} \mathrm{O}\right)$, tetrahydrofuran $(\mathrm{THF})$, benzene $\left(\mathrm{C}_{6} \mathrm{H}_{6}\right)$ were dried using a GlassContours drying column and stored over $4 \AA$ molecular sieves. Benzene- $d_{6}$ (Cambridge Isotopes) was dried over sodium-benzophenone ketyl, distilled, and stored over $4 \AA$ molecular sieves. Toluene- $d_{8}$ (Cambridge Isotopes) was dried over $\mathrm{CaH}_{2}$, vacuum transferred, and stored over $4 \AA$ molecular sieves. Chlorofom- $d_{1}$ (Cambridge Isotopes) was dried over copper(II) sulfate/calcium chloride vacuum transferred, and stored over $4 \AA$ molecular sieves. Norbornene was refluxed over sodium, distilled, and stored under argon. Benzylmagnesium chloride (1 M in $\mathrm{Et}_{2} \mathrm{O}$, and $2 \mathrm{M}$ in $\mathrm{THF}$ ), trimethylsilylmethylmagnesium chloride (1 $\mathrm{M}$ in $\mathrm{Et}_{2} \mathrm{O}$ ), and 2-methyl-2phenylpropylmagnesium chloride $\left(0.5 \mathrm{M}_{\text {in }} \mathrm{Et}_{2} \mathrm{O}\right)$ were purchased from Sigma-Aldrich and used as received. Lithium tert-butoxide $(98+\%)$ was purchased from Strem Chemicals and used as received. $\left[\mathrm{CF}_{3}-\mathrm{ONO}\right] \mathrm{H}_{3}(\mathbf{1})^{1}$ and $\left[\mathrm{CF}_{3}-\mathrm{ONO}\right] \mathrm{TaCl}_{2}(\mathrm{THF})(\mathbf{8})^{2}$ were prepared according to reported procedures. All other reagents were purchased from commercial vendors and used without further purification. Elemental analyses were performed at Complete Analysis Laboratory Inc., Parsippany, New Jersey.

\section{NMR spectroscopy characterization}

NMR spectra were obtained on a Varian Mercury spectrometer operating at $300 \mathrm{MHz}$ for ${ }^{1} \mathrm{H}$, or a Varian Inova spectrometer operating at $500 \mathrm{MHz}$ for ${ }^{1} \mathrm{H}$. The chemical shifts are reported in $\delta$ (ppm), referenced to the lock signal on the TMS scale for ${ }^{1} \mathrm{H}$ and ${ }^{13} \mathrm{C} \mathrm{NMR} \mathrm{spectra},{ }^{3} \mathrm{CFCl}_{3}$ for 
${ }^{19} \mathrm{~F}$ NMR spectra, and neat $\mathrm{H}_{3} \mathrm{PO}_{4}$ scale for ${ }^{31} \mathrm{P}$ NMR spectra. Compounds 2-9 were characterized by ${ }^{1} \mathrm{H},{ }^{13} \mathrm{C}$, and ${ }^{19} \mathrm{~F}$ NMR spectroscopy. The chemical shifts are presented in Error! Reference source not found.. The assignments were primarily based on the cross-peaks observed in the ${ }^{1} \mathrm{H}-{ }^{13} \mathrm{C} g \mathrm{HMBC}$ spectra. The spectra were recorded at $25{ }^{\circ} \mathrm{C}$ unless noted otherwise. 


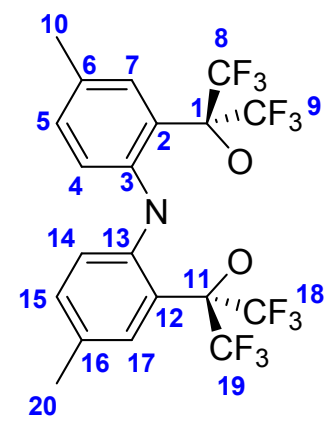

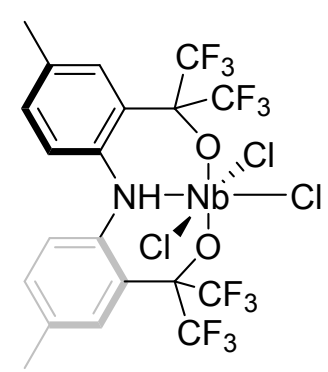

2

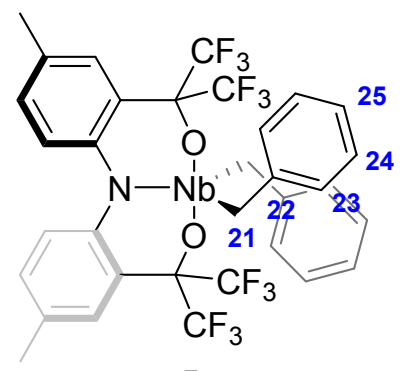

5

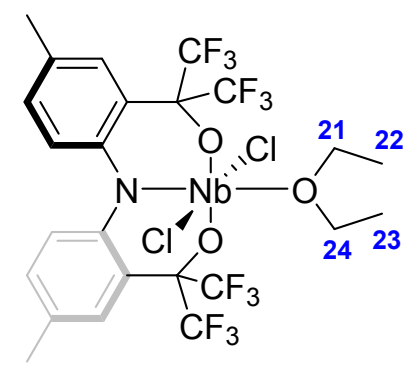

3

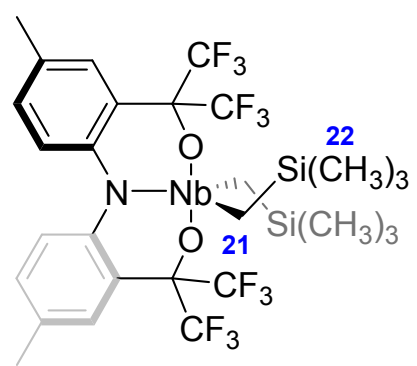

6

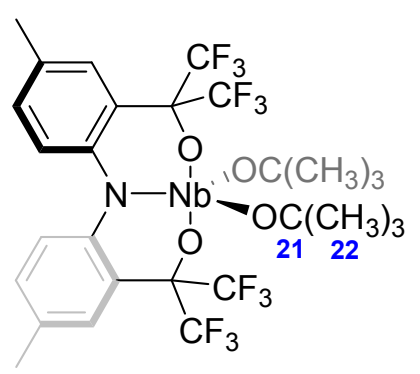

4

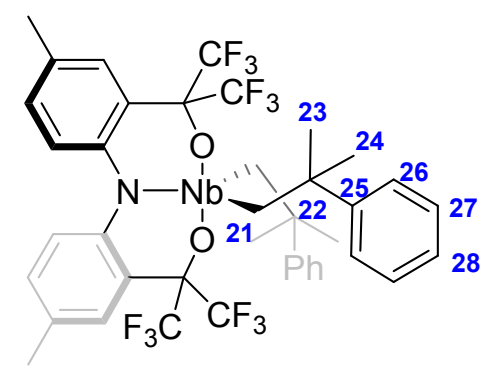

7

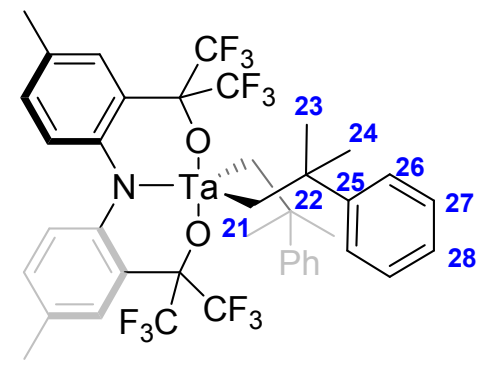

9

Figure S1. Labeling scheme for ${ }^{1} \mathrm{H},{ }^{13} \mathrm{C}$ and ${ }^{19} \mathrm{~F}$ NMR resonances of complexes 2 - 9 
Table S1. ${ }^{1} \mathrm{H},{ }^{13} \mathrm{C}$, and ${ }^{19} \mathrm{~F}$ chemical shifts in compounds 2 -9

\begin{tabular}{|c|c|c|c|c|c|c|c|c|}
\hline Compd. & 1 & $2^{a}$ & 3-Et $\mathrm{t}_{2} \mathrm{O}$ & 4 & 5 & 6 & 7 & 9 \\
\hline $\mathrm{H} 4$ & 6.69 & 6.27 & 6.40 & 6.77 & 6.61 & 6.82 & 6.81 & 6.76 \\
\hline H5 & 6.63 & 7.17 & 6.61 & 6.72 & 6.66 & 6.70 & 6.71 & 6.71 \\
\hline $\mathrm{H} 7$ & 7.47 & 7.50 & 7.62 & 7.63 & 7.44 & 7.57 & 7.56 & 7.54 \\
\hline H10 & 1.83 & 2.40 & 1.91 & 1.96 & 1.97 & 1.92 & 1.94 & 1.94 \\
\hline H14 & 6.69 & 7.02 & 6.40 & 6.77 & 6.61 & 6.82 & 6.81 & 6.76 \\
\hline H15 & 6.63 & 7.45 & 6.61 & 6.72 & 6.66 & 6.70 & 6.71 & 6.71 \\
\hline H17 & 7.47 & 7.74 & 7.62 & 7.63 & 7.44 & 7.57 & 7.56 & 7.54 \\
\hline $\mathrm{H} 20$ & 1.83 & 2.54 & 1.91 & 1.96 & 1.97 & 1.92 & 1.94 & 1.94 \\
\hline $\mathrm{H} 21$ & - & - & 3.90 & - & 2.52 & $2.21^{b}$ & $2.38^{c}$ & $1.91^{d}$ \\
\hline $\mathrm{H} 22$ & - & - & 1.06 & 1.18 & - & 0.05 & - & \\
\hline $\mathrm{H} 23$ & - & - & - & - & 6.92 & - & 1.20 & 1.24 \\
\hline $\mathrm{H} 24$ & - & - & - & - & 6.89 & - & 1.31 & 1.33 \\
\hline $\mathrm{H} 25$ & - & - & - & - & 6.87 & - & - & \\
\hline $\mathrm{H} 26$ & - & - & - & - & - & - & 7.24 & 7.22 \\
\hline $\mathrm{H} 27$ & - & - & - & - & - & - & 7.12 & 7.11 \\
\hline $\mathrm{H} 28$ & - & - & - & - & - & - & 7.00 & 6.99 \\
\hline $\mathrm{C} 1$ & 80.6 & 87.7 & 85.0 & 83.1 & 83.6 & 84.1 & 84.1 & 84.2 \\
\hline $\mathrm{C} 2$ & 120.9 & 123.4 & 124.1 & 124.7 & 123.3 & 124.6 & 124.9 & 124.5 \\
\hline $\mathrm{C} 3$ & 143.0 & 143.1 & 148.6 & 148.6 & 148.8 & 147.6 & 147.8 & 147.1 \\
\hline $\mathrm{C} 4$ & 126.1 & 121.7 & 123.0 & 125.4 & 125.6 & 126.7 & 126.5 & 126.6 \\
\hline $\mathrm{C} 5$ & 132.1 & 132.2 & 131.9 & 131.7 & 131.9 & 132.3 & 132.3 & 132.4 \\
\hline C6 & 134.4 & 137.1 & 136.4 & 131.0 & 131.1 & 131.5 & 131.6 & 131.9 \\
\hline $\mathrm{C} 7$ & 128.3 & 128.7 & 126.9 & 126.8 & 126.4 & 126.9 & 126.9 & 126.8 \\
\hline $\mathrm{C} 8$ & 123.2 & $\mathrm{~nm}$ & $\mathrm{~nm}$ & $\mathrm{~nm}$ & $\mathrm{~nm}$ & $\mathrm{~nm}$ & $\mathrm{~nm}$ & $\mathrm{~nm}$ \\
\hline C9 & 123.3 & $\mathrm{~nm}$ & $\mathrm{~nm}$ & $\mathrm{~nm}$ & $\mathrm{~nm}$ & $\mathrm{~nm}$ & $\mathrm{~nm}$ & $\mathrm{~nm}$ \\
\hline $\mathrm{C} 10$ & 20.2 & 21.1 & 20.5 & 20.2 & 20.1 & 20.2 & 20.2 & 20.1 \\
\hline $\mathrm{C} 11$ & 80.6 & 85.4 & 85.0 & 83.1 & 83.6 & 84.1 & 84.1 & 84.2 \\
\hline $\mathrm{C} 12$ & 120.9 & 127.2 & 124.1 & 124.7 & 123.3 & 124.6 & 124.9 & 124.5 \\
\hline $\mathrm{C} 13$ & 143.0 & 133.5 & 148.6 & 148.6 & 148.8 & 147.6 & 147.8 & 147.1 \\
\hline C14 & 126.1 & 130.1 & 123.0 & 125.4 & 125.6 & 126.7 & 126.5 & 126.6 \\
\hline $\mathrm{C} 15$ & 132.1 & 133.5 & 131.9 & 131.7 & 131.9 & 132.3 & 132.3 & 132.4 \\
\hline $\mathrm{C} 16$ & 134.4 & 141.0 & 136.4 & 131.0 & 131.1 & 131.5 & 131.6 & 131.9 \\
\hline $\mathrm{C} 17$ & 128.3 & 129.0 & 126.9 & 126.8 & 126.4 & 126.9 & 126.9 & 126.8 \\
\hline $\mathrm{C} 18$ & 123.2 & $\mathrm{~nm}$ & $\mathrm{~nm}$ & $\mathrm{~nm}$ & $\mathrm{~nm}$ & $\mathrm{~nm}$ & $\mathrm{~nm}$ & $\mathrm{~nm}$ \\
\hline C19 & 123.3 & $\mathrm{~nm}$ & $\mathrm{~nm}$ & $\mathrm{~nm}$ & $\mathrm{~nm}$ & $\mathrm{~nm}$ & $\mathrm{~nm}$ & $\mathrm{~nm}$ \\
\hline $\mathrm{C} 20$ & 20.2 & 21.5 & 20.5 & 20.2 & 20.1 & 20.2 & 20.2 & 20.1 \\
\hline $\mathrm{C} 21$ & - & - & 70.7 & 88.3 & 68.8 & 77.4 & 99.1 & 105.1 \\
\hline
\end{tabular}




\begin{tabular}{lllllllll} 
C22 & - & - & 13.0 & 30.0 & 132.4 & 0.7 & 41.9 & 42.1 \\
C23 & - & - & 13.0 & - & 132.7 & - & 32.0 & 33.1 \\
C24 & - & - & 70.7 & - & 129.5 & - & 31.8 & 32.9 \\
C25 & - & - & - & - & 127.6 & - & 150.0 & 151.1 \\
C26 & - & - & - & - & - & - & 125.6 & 125.4 \\
C27 & - & - & - & - & - & - & 128.4 & 128.3 \\
C28 & - & - & - & - & - & - & 126.0 & 125.9 \\
\hline F8 & -74.2 & -68.4 & -68.9 & -71.2 & -70.4 & -70.6 & -69.8 & -70.0 \\
F9 & -75.7 & -69.7 & -73.5 & -75.5 & -74.9 & -75.1 & -74.4 & -74.7 \\
F18 & -74.2 & -70.9 & -68.9 & -71.2 & -70.4 & -70.6 & -69.8 & -70.0 \\
F19 & -75.7 & -72.4 & -73.5 & -75.5 & -74.9 & -75.1 & -74.4 & -74.7 \\
\hline
\end{tabular}

$a$ - measured in $\mathrm{CDCl}_{3}$, [ONHO$] \mathrm{NbCl}_{3}$ resonates at $7.50 \mathrm{ppm}\left(\mathrm{q} ; \mathrm{J}_{H---F}=\sim 6.4 \mathrm{~Hz}\right.$ in $\mathrm{C}_{6} \mathrm{D}_{6}, 25$ $\left.{ }^{\circ} \mathrm{C}\right) ; b$ - diastereotopic peaks: $2.21 \mathrm{ppm}$ and $2.14 \mathrm{ppm} ; c$-diastereotopic peaks: $2.38 \mathrm{ppm}$ and $2.11 \mathrm{ppm} ; d$-diastereotopic peaks: $1.91 \mathrm{ppm}$ and $1.71 \mathrm{ppm}$

All spectra were obtained in $\mathrm{C}_{6} \mathrm{D}_{6}$ at $25^{\circ} \mathrm{C}$, unless noted otherwise.

\section{Synthesis of $\left[\mathrm{CF}_{3}-\mathrm{ONHO}\right] \mathrm{NbCl}_{3}(2)$}

In an argon-filled glove box, $\left[\mathrm{CF}_{3}-\mathrm{ONO}\right] \mathrm{H}_{3}(0.100 \mathrm{~g}, 0.189 \mathrm{mmol})$ was dissolved in $2 \mathrm{~mL}$ of benzene in a vial. In a second vial, $\mathrm{NbCl}_{5}(0.051 \mathrm{~g}, 0.189 \mathrm{mmol})$ was also dissolved in $2 \mathrm{~mL}$ of benzene. The $\left[\mathrm{CF}_{3}-\mathrm{ONO}\right] \mathrm{H}_{3}$ solution was added dropwise to the $\mathrm{NbCl}_{5}$ solution over the course of a few minutes, resulting in a dark brown reaction mixture. The solution was stirred for $4 \mathrm{~h}$. After $4 \mathrm{~h}$, the solution was filtered through a celite pad; all volatiles were then removed in vacuo affording a pale brown solid. (Yield $=0.126 \mathrm{~g}, 81.2 \%$ )

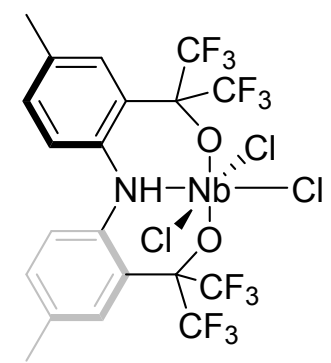


${ }^{1} \mathrm{H}$ NMR $\left(500 \mathrm{MHz}, \mathrm{CDCl}_{3}\right) \delta 7.74(\mathrm{~s}, 1 \mathrm{H}, \mathrm{Ar}-H), 7.55-7.47$ (m, 1H, Ar- $\left.H ; \mathrm{N}_{\text {pincer }}-H\right), 7.44$ (d, $\left.1 \mathrm{H},{ }^{3} J_{\mathrm{HH}}=8.2 \mathrm{~Hz}, \operatorname{Ar}-H\right), 7.16\left(\mathrm{~d}, 1 \mathrm{H},{ }^{3} J_{\mathrm{HH}}=8.3 \mathrm{~Hz}, \operatorname{Ar}-H\right), 7.01\left(\mathrm{~d}, 1 \mathrm{H},{ }^{3} J_{\mathrm{HH}}=8.4 \mathrm{~Hz}, \operatorname{Ar}-H\right)$, $6.26\left(\mathrm{~d}, 1 \mathrm{H},{ }^{3} J_{\mathrm{HH}}=8.6 \mathrm{~Hz}, \mathrm{Ar}-\mathrm{H}\right), 2.54\left(\mathrm{~s}, 3 \mathrm{H}, \mathrm{Ar}-\mathrm{CH}_{3}\right), 2.40\left(\mathrm{~s}, 3 \mathrm{H}, \mathrm{Ar}-\mathrm{CH}_{3}\right)$.

${ }^{13} \mathrm{C}\left\{{ }^{1} \mathrm{H}\right\}$ NMR (126 MHz, $\left.\mathrm{CDCl}_{3}\right) \delta 143.3(\mathrm{Ar}-\mathrm{C}), 141.1$ (Ar-C), $137.4(\mathrm{Ar}-\mathrm{C}), 133.6(\mathrm{Ar}-\mathrm{C})$, $132.3(\mathrm{Ar}-C), 130.2(\mathrm{Ar}-C), 129.2(\mathrm{Ar}-C), 128.9$ (Ar-C), 128.5 (Ar-C), $127.3(\mathrm{Ar}-C), 123.6(\mathrm{Ar}-$ C), $121.9(\mathrm{Ar}-\mathrm{C}), 87.9\left(\left(\mathrm{CF}_{3}\right)_{2} \mathrm{CO}-\right), 85.3\left(\left(\mathrm{CF}_{3}\right)_{2} \mathrm{CO}-\right), 21.6\left(\mathrm{CH}_{3}\right), 21.1\left(\mathrm{CH}_{3}\right) \mathrm{ppm}$.

${ }^{19} \mathrm{~F} \mathrm{NMR}\left(282 \mathrm{MHz}, \mathrm{CDCl}_{3}\right) \delta-68.4\left(\mathrm{q}, 3 \mathrm{~F},{ }^{4} J_{\mathrm{FF}}=10.5 \mathrm{~Hz}\right),-69.7\left(\mathrm{q}, 3 \mathrm{~F},{ }^{4} J_{\mathrm{FF}}=9.2 \mathrm{~Hz}\right),-70.9$ $\left(\mathrm{q}, 3 \mathrm{~F},{ }^{4} J_{\mathrm{FF}}=9.3 \mathrm{~Hz}\right),-72.4\left(\mathrm{dq}, 3 \mathrm{~F},{ }^{4} J_{\mathrm{FF}}=10.4, J_{\mathrm{H} \cdots \mathrm{F}}=5.6 \mathrm{~Hz}\right)$.

Anal. Calcd. for $\mathrm{C}_{20} \mathrm{H}_{13} \mathrm{Cl}_{3} \mathrm{~F}_{12} \mathrm{NNbO}_{2}(726.57 \mathrm{~g} / \mathrm{mol})$ : C, 33.06\%; H, $1.80 \%$; N, 1.93\%. Found: $\mathrm{C}, 32.79 \%, \mathrm{H}, 1.82 \%, \mathrm{~N}, 2.13 \%$ 


\section{NMR Characterization of $\left[\mathrm{CF}_{3}-\mathrm{ONHO}\right] \mathrm{NbCl}_{3}(2)$}

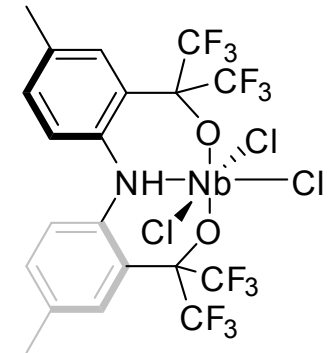

2

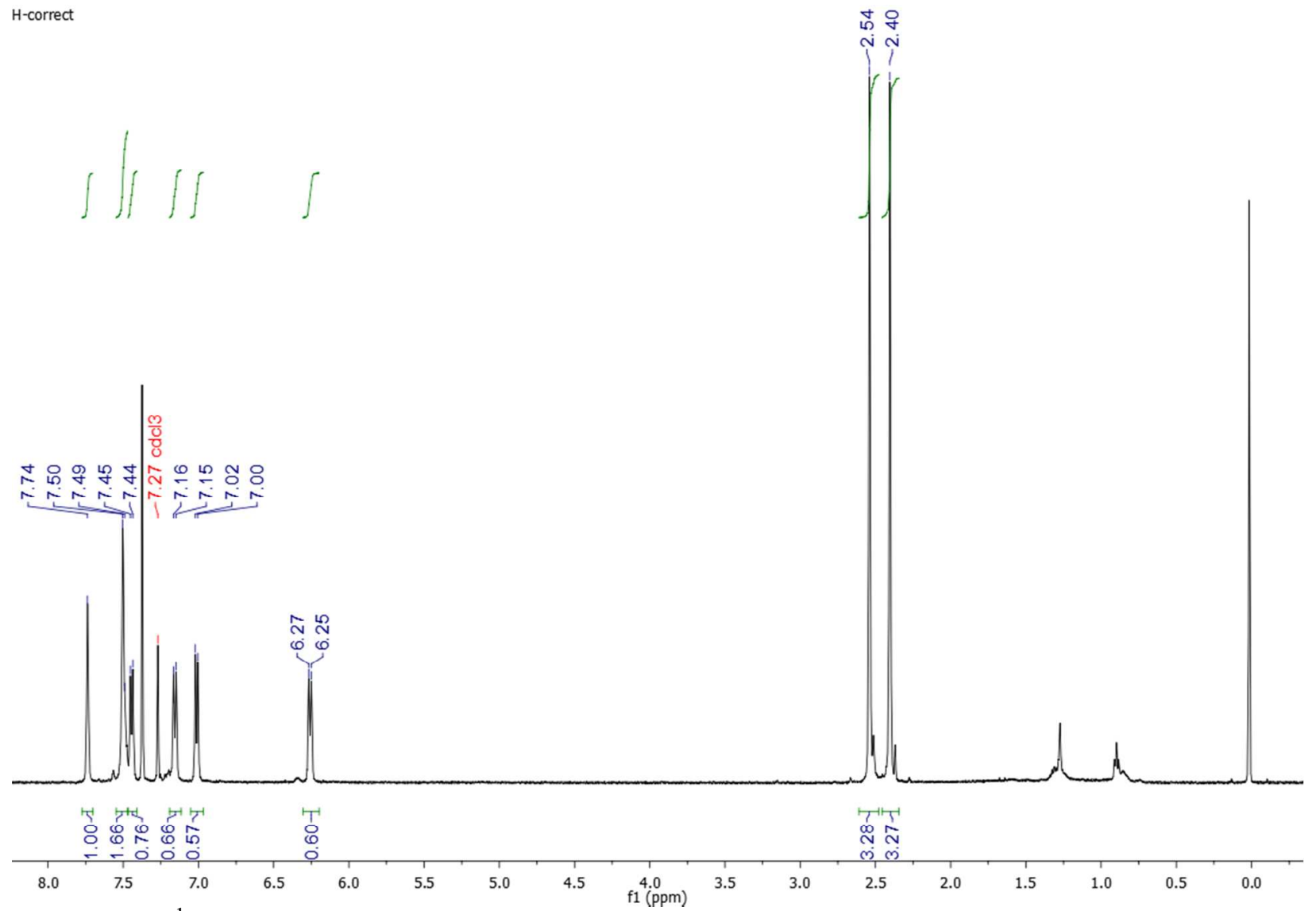

Figure S2. ${ }^{1} \mathrm{H} \mathrm{NMR}$ spectrum of $2\left(\mathrm{CDCl}_{3}, 500 \mathrm{MHz}, 25^{\circ} \mathrm{C}\right)$ 

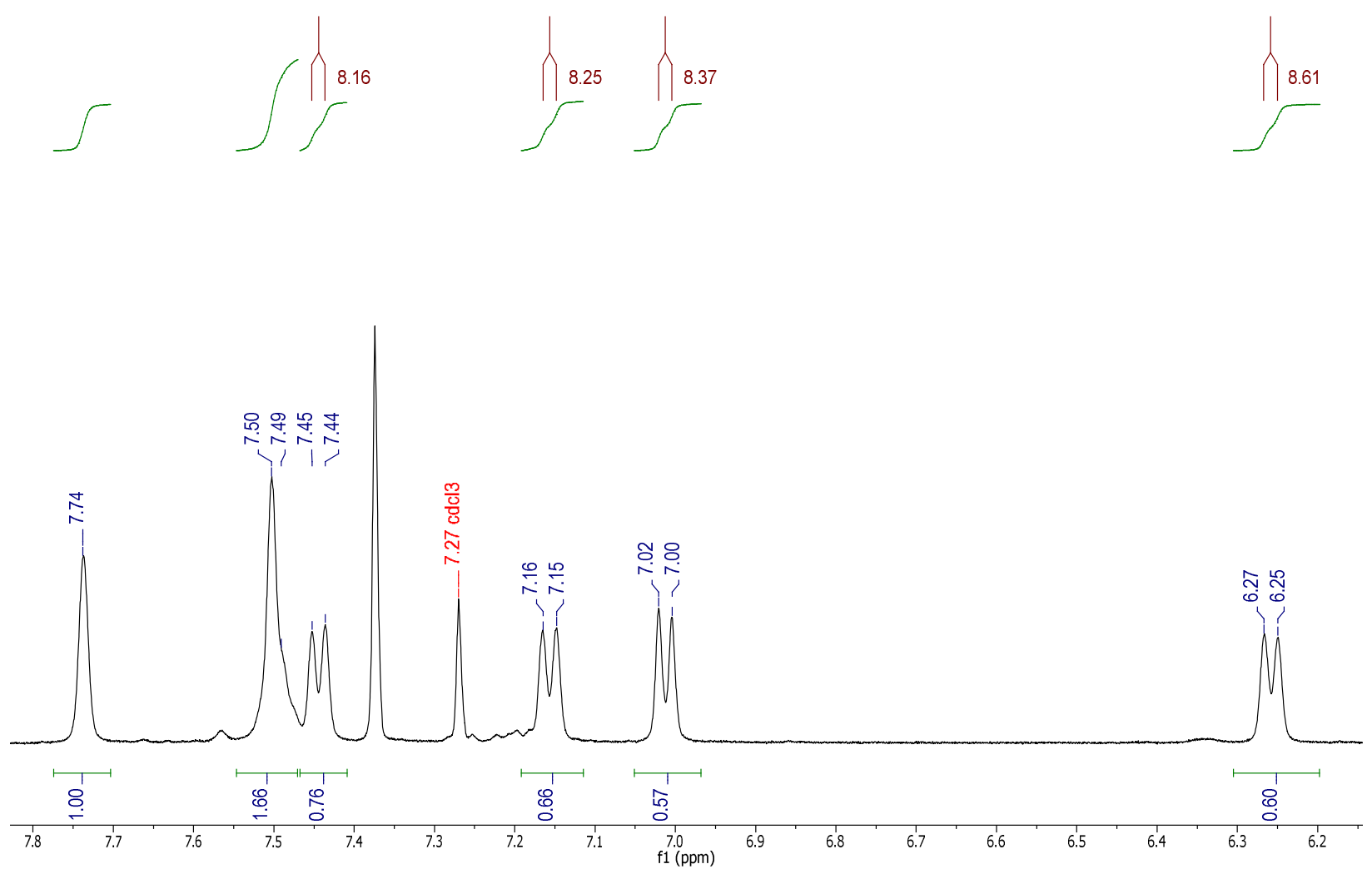

Figure S3. ${ }^{1} \mathrm{H}$ NMR spectrum of 2 - Expansion $\left(\mathrm{CDCl}_{3}, 500 \mathrm{MHz}, 25^{\circ} \mathrm{C}\right)$ 


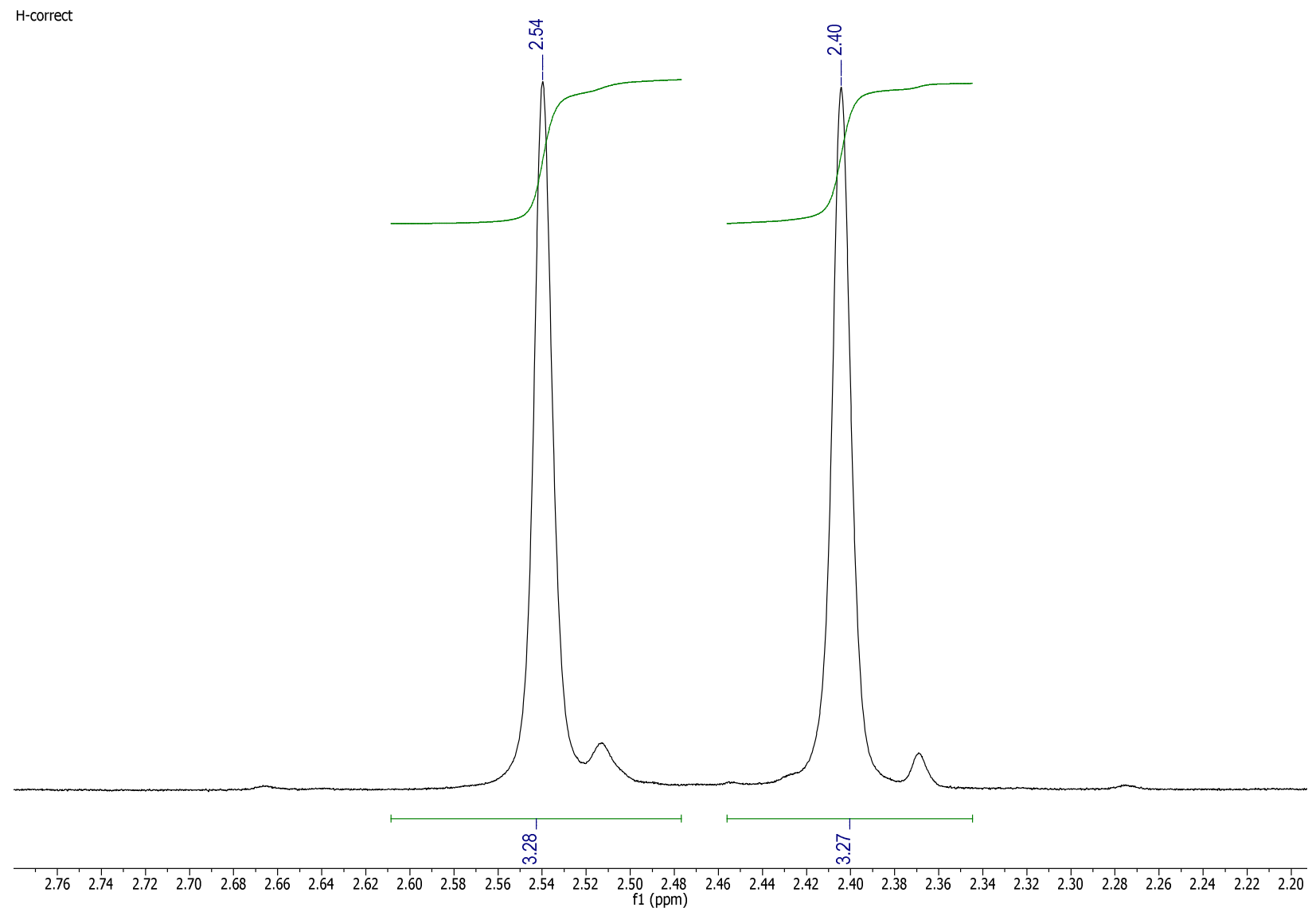

Figure S4. ${ }^{1} \mathrm{H}$ NMR spectrum of 2 - Expansion $\left(\mathrm{CDCl}_{3}, 500 \mathrm{MHz}, 25{ }^{\circ} \mathrm{C}\right)$ 


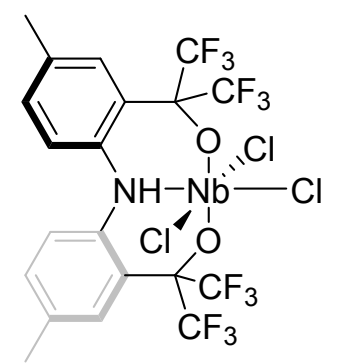

2

SUd_UNHUINBCI3-CDCI3-_LOUOUSCans_13C
new experiment

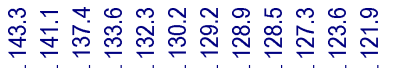

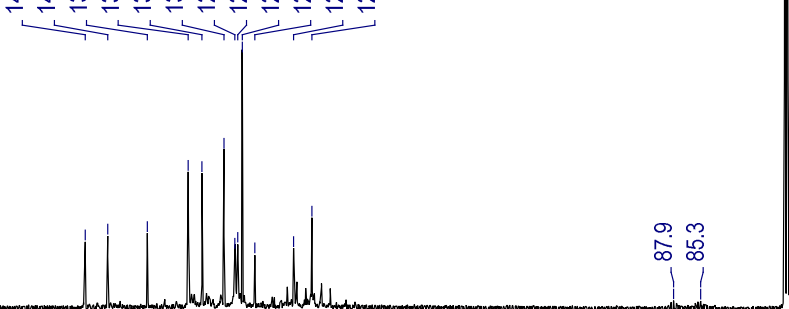

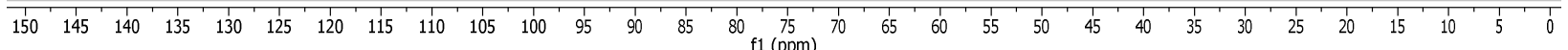

Figure S5. ${ }^{13} \mathrm{C}\left\{{ }^{1} \mathrm{H}\right\}$ NMR spectrum of $2\left(\mathrm{CDCl}_{3}, 126 \mathrm{MHz}, 25{ }^{\circ} \mathrm{C}\right)$ 


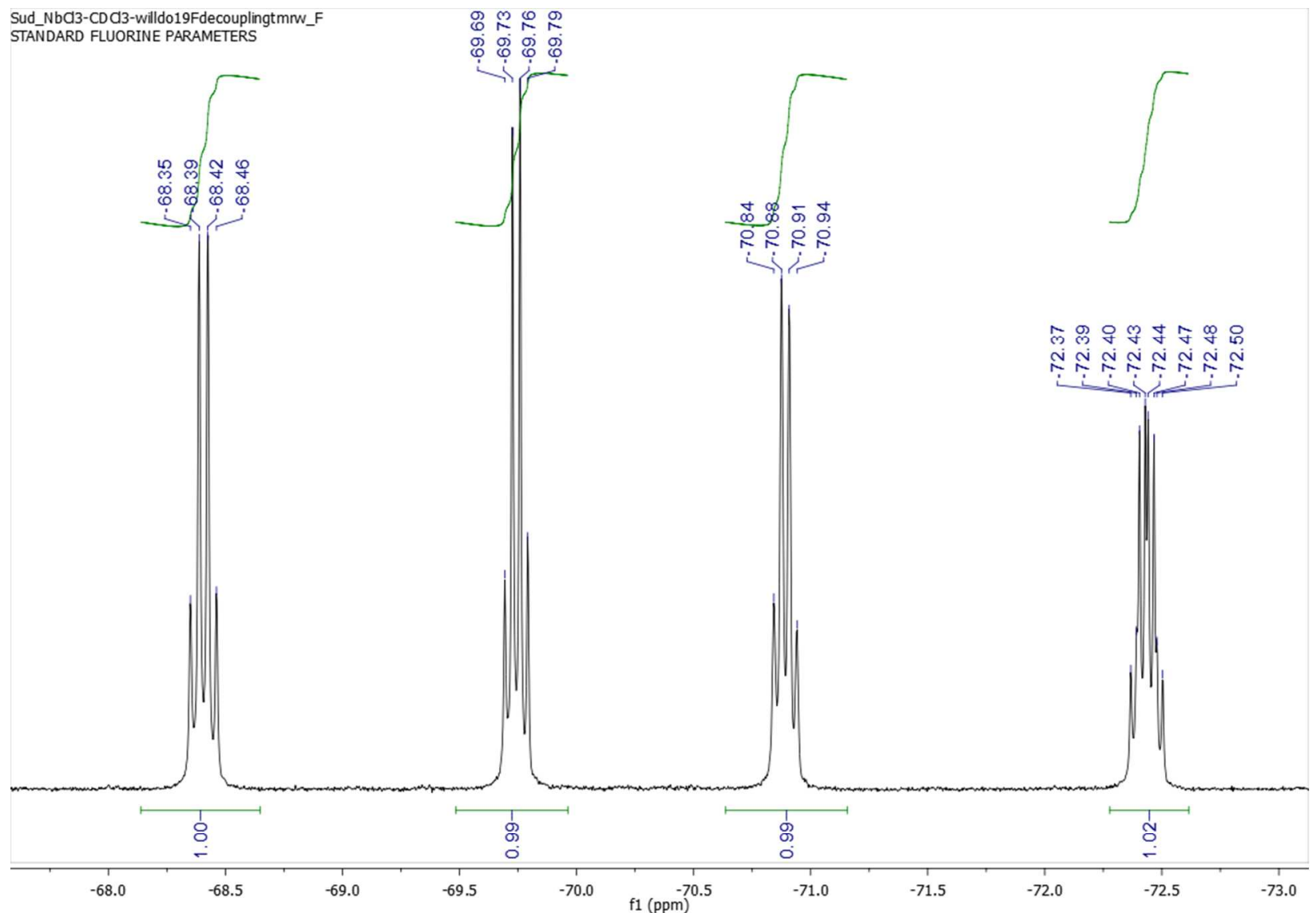

Figure S6. ${ }^{19} \mathrm{~F}$ NMR spectrum of $2\left(\mathrm{CDCl}_{3}, 282 \mathrm{MHz}, 25{ }^{\circ} \mathrm{C}\right)$
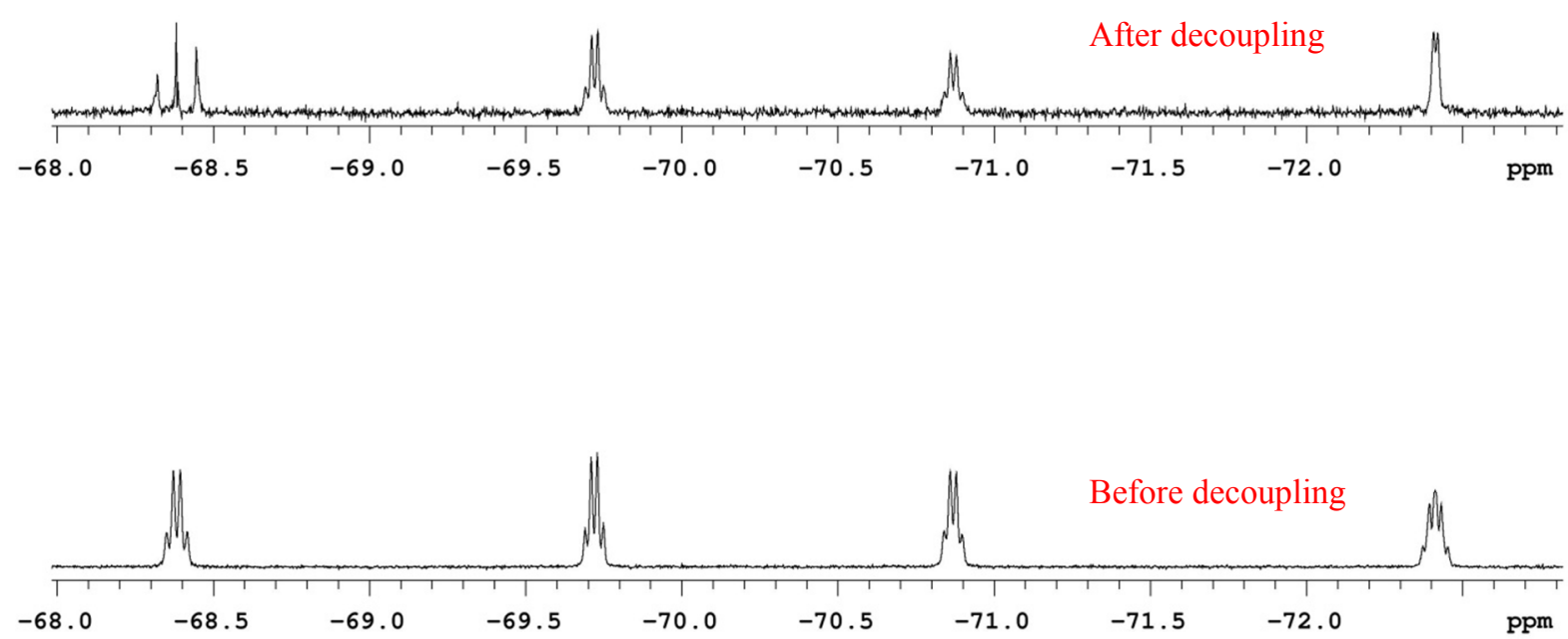

Figure S7. ${ }^{19} \mathrm{~F}$ NMR spectrum of $2\left(\mathrm{CDCl}_{3}, 470 \mathrm{MHz}, 25{ }^{\circ} \mathrm{C}\right)$ - Homonuclear Selective Decoupling at $-68.4 \mathrm{ppm}$ resolves doublet of quartets at -72.4 into a doublet $\left(J_{\mathrm{H} \cdots \mathrm{F}}=6.6 \mathrm{~Hz}\right)$ 


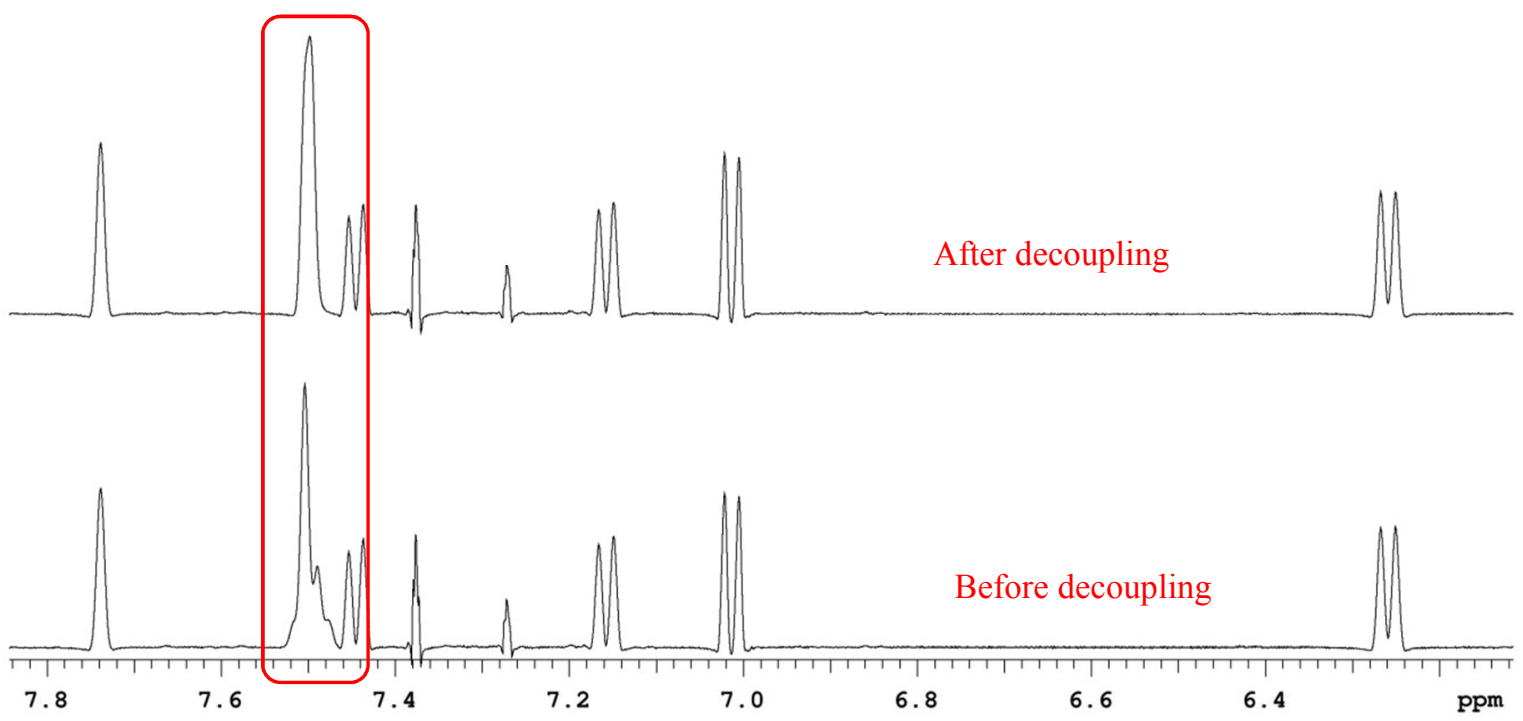

Figure S8. ${ }^{1} \mathrm{H}$ NMR spectrum of $\mathbf{2}$ - Expansion of the aromatic region $\left(\mathrm{CDCl}_{3}, 500 \mathrm{MHz}, 25\right.$ ${ }^{\circ} \mathrm{C}$ ) - Decoupling the $-\mathrm{CF}_{3}$ resonance at $-72.4 \mathrm{ppm}$ in the ${ }^{19} \mathrm{~F}$ spectrum resolves the $-\mathrm{N}_{\text {pincer }} H$ resonance at ca. $7.49 \mathrm{ppm}$ (quartet) into a singlet. The $-\mathrm{N}_{\text {pincer }} H$ resonance overlaps with an aromatic proton at $7.50 \mathrm{ppm}$, appearing as a broad "singlet" integrating to $2 \mathrm{H}$.

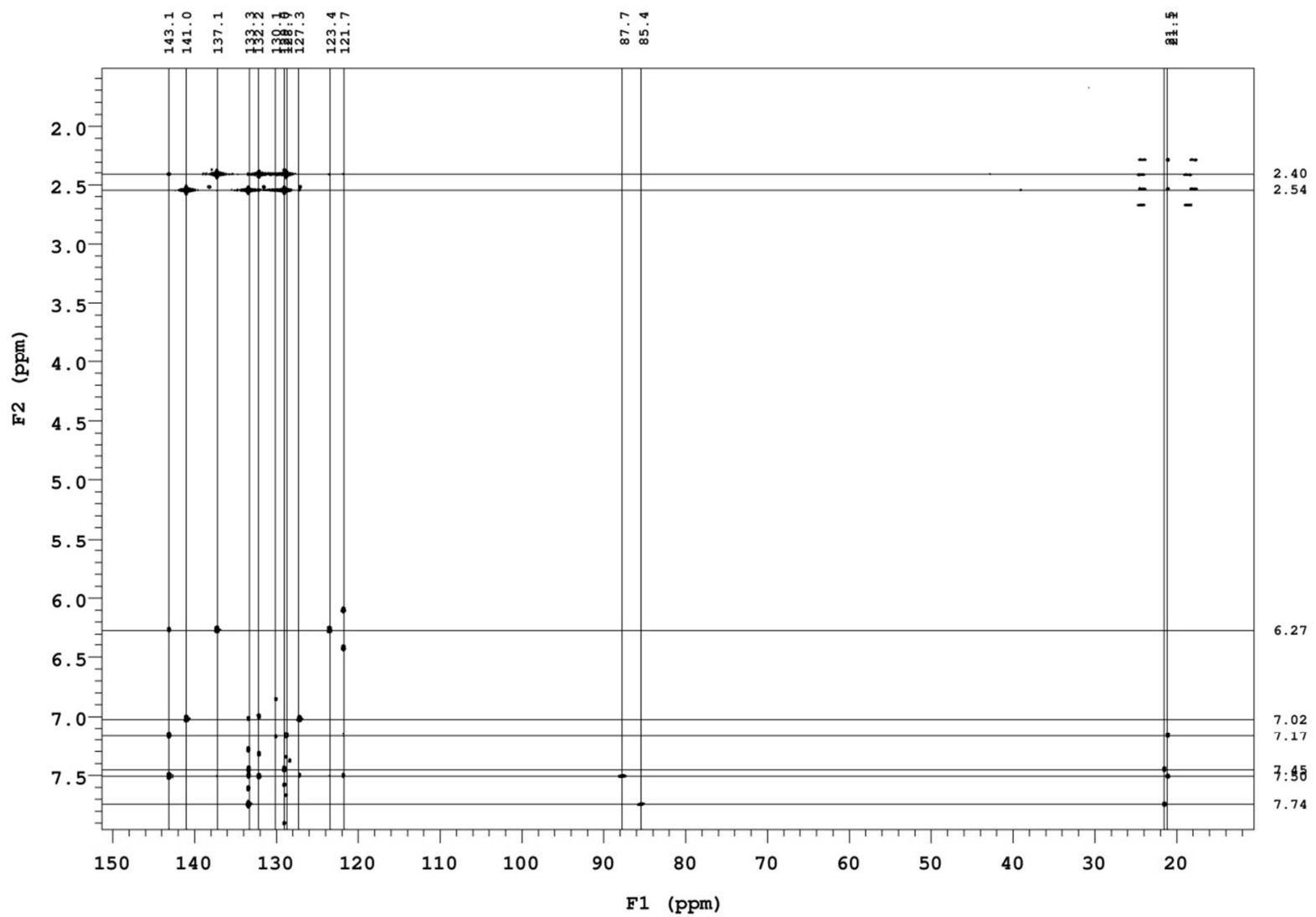

Figure S9. ${ }^{1} \mathrm{H}^{13} \mathrm{C} g \mathrm{HMBC}$ spectrum of $2\left(\mathrm{CDCl}_{3}, 500 \mathrm{MHz}, 25{ }^{\circ} \mathrm{C}\right)$ 


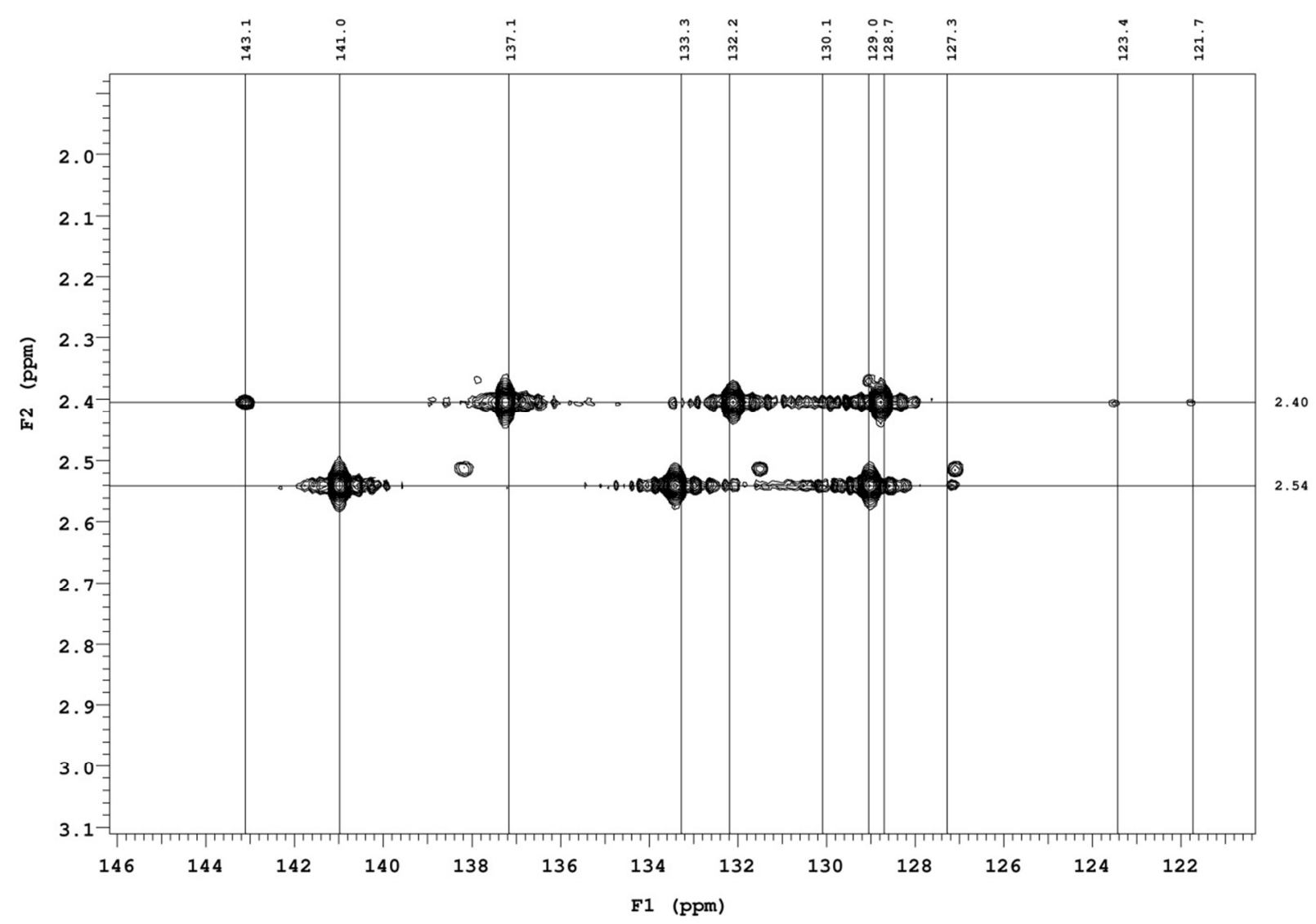

Figure S10. ${ }^{1} \mathrm{H}_{-}{ }^{13} \mathrm{C} \mathrm{gHMBC}$ spectrum of 2 - Expansion $\left(\mathrm{CDCl}_{3}, 500 \mathrm{MHz}, 25{ }^{\circ} \mathrm{C}\right)$ 


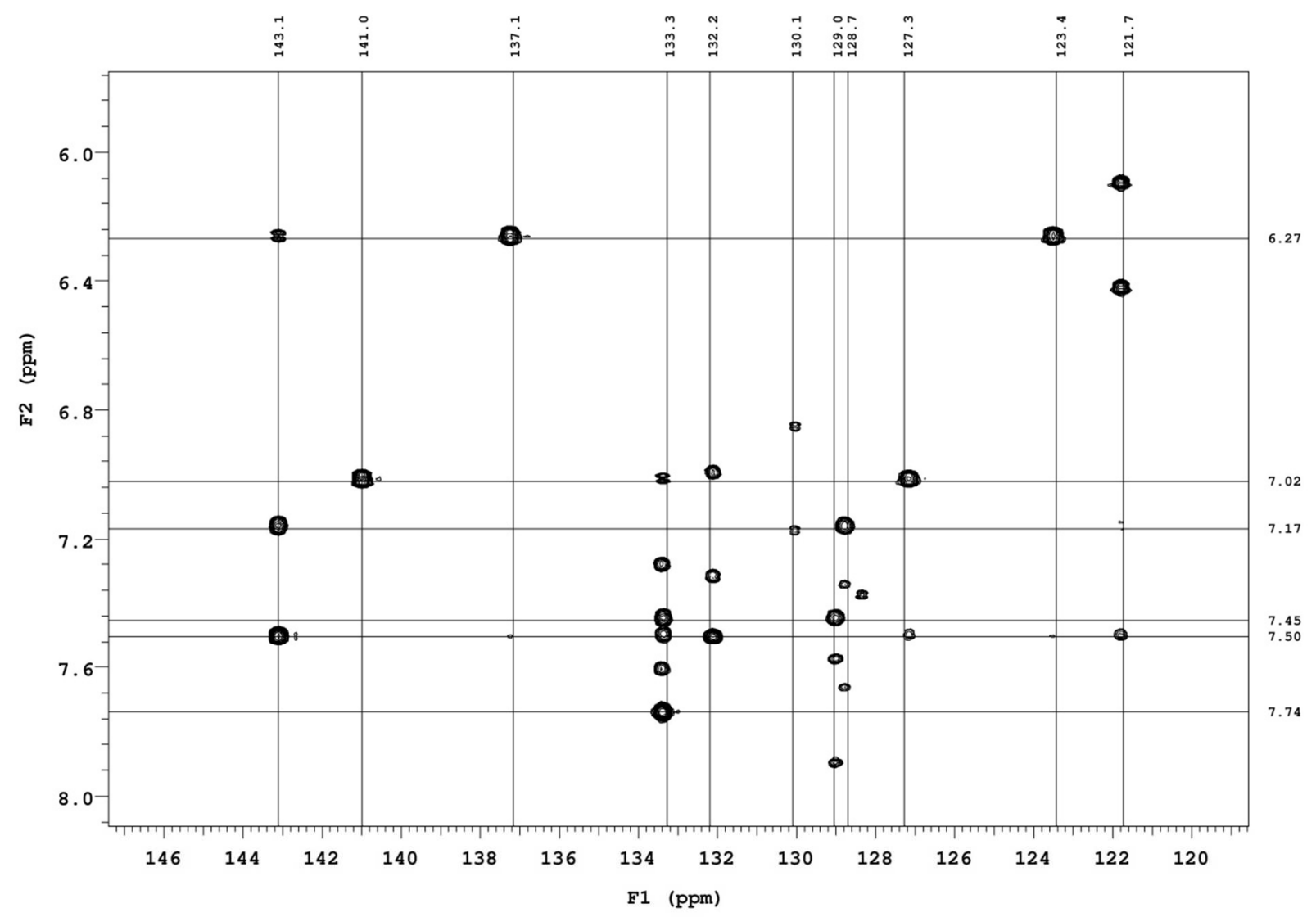

Figure S11. ${ }^{1} \mathrm{H}-{ }^{13} \mathrm{C} g \mathrm{HMBC}$ spectrum of 2 - Expansion $\left(\mathrm{CDCl}_{3}, 500 \mathrm{MHz}, 25{ }^{\circ} \mathrm{C}\right)$ 


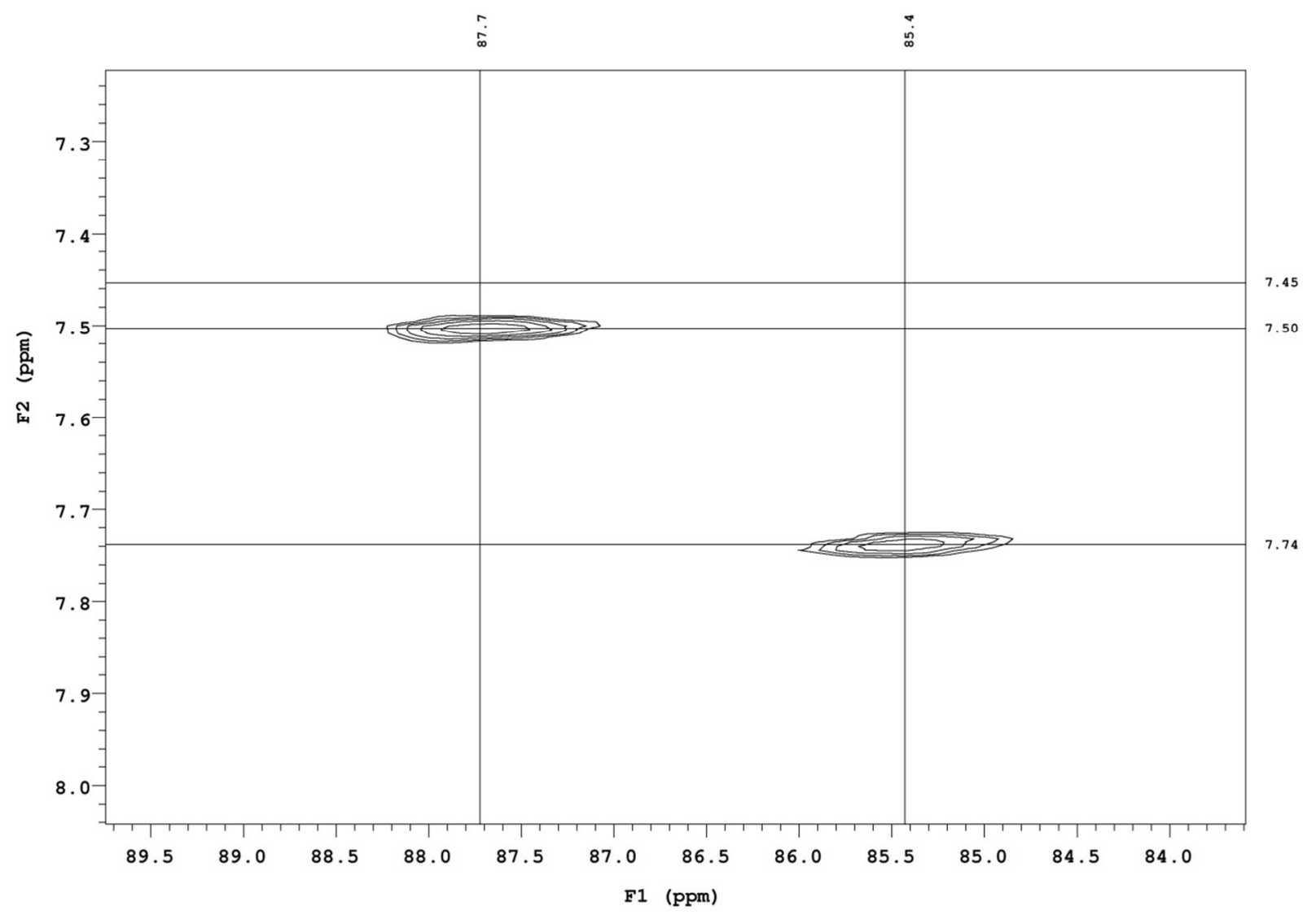

Figure S12. ${ }^{1} \mathrm{H}^{-13} \mathrm{C}$ gHMBC spectrum of $\mathbf{2}-$ Expansion $\left(\mathrm{CDCl}_{3}, 500 \mathrm{MHz}, 25{ }^{\circ} \mathrm{C}\right)$ 


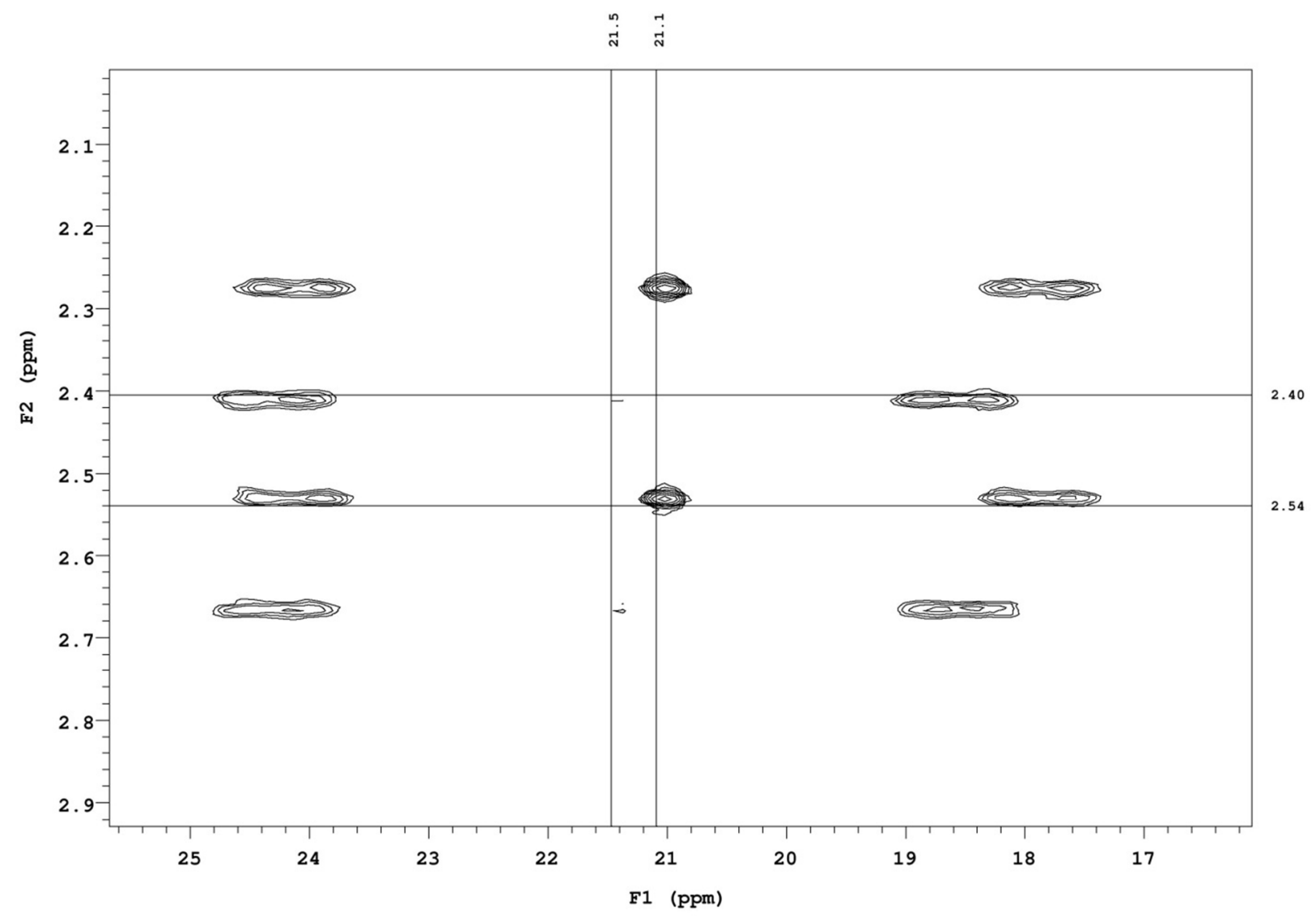

Figure S13. ${ }^{1} \mathrm{H}-{ }^{13} \mathrm{C} g \mathrm{HMBC}$ spectrum of 2 - Expansion $\left(\mathrm{CDCl}_{3}, 500 \mathrm{MHz}, 25{ }^{\circ} \mathrm{C}\right)$ 


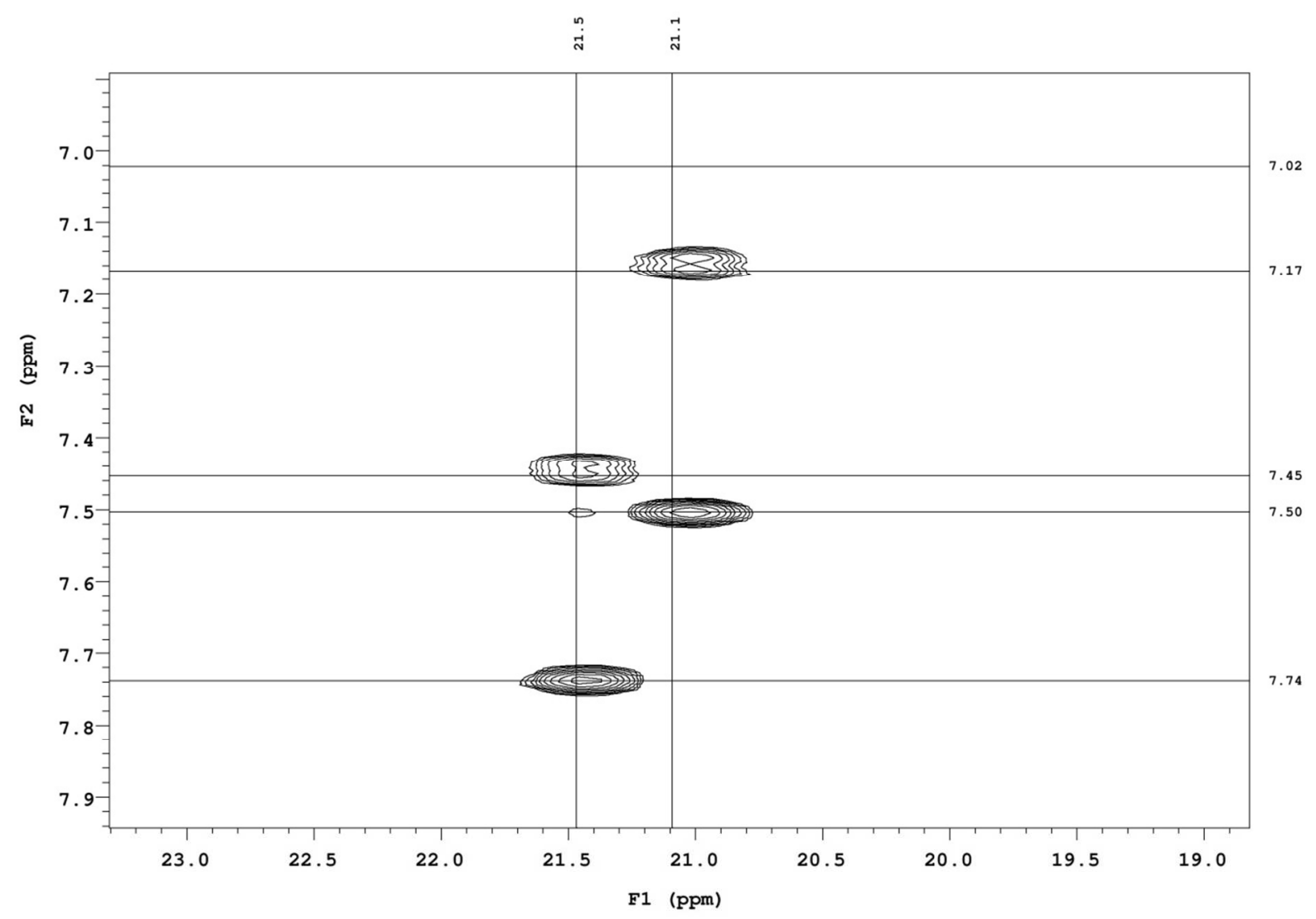

Figure S14. ${ }^{1} \mathrm{H}-{ }^{13} \mathrm{C} g \mathrm{HMBC}$ spectrum of 2 - Expansion $\left(\mathrm{CDCl}_{3}, 500 \mathrm{MHz}, 25{ }^{\circ} \mathrm{C}\right)$ 


\section{Synthesis of $\left[\mathrm{CF}_{3}-\mathrm{ONO}\right] \mathrm{NbCl}_{2}\left(\mathrm{Et}_{2} \mathrm{O}\right)\left(3-\mathrm{Et}_{2} \mathrm{O}\right)$}

The complex 3-Et $\mathbf{2} \mathbf{O}$ can be synthesized in two ways: with or without isolation of 2 . The procedure described herein involves conversion of $\mathbf{2}$ to $\mathbf{3}-\mathbf{E} \mathbf{t}_{\mathbf{2}} \mathbf{O}$.

In a glovebox, $2(0.128 \mathrm{~g}, 0.176 \mathrm{mmol})$ was dissolved in $2 \mathrm{~mL} \mathrm{C}_{6} \mathrm{H}_{6}$ and a pipet of diethyl ether $\left(\mathrm{Et}_{2} \mathrm{O}\right)$ was added. The initially brown colored solution in $\mathrm{C}_{6} \mathrm{H}_{6}$ changes to maroon over $6 \mathrm{~h}$.

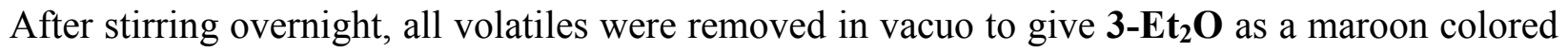
solid. (Yield $=0.117 \mathrm{~g}, 86.9 \%$ )

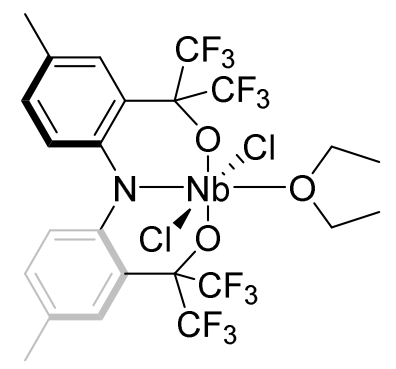

$3-\mathrm{Et}_{\mathbf{2}} \mathrm{O}$

${ }^{1} \mathrm{H}$ NMR $\left(500 \mathrm{MHz}, \mathrm{C}_{6} \mathrm{D}_{6}\right) \delta 7.62(\mathrm{~s}, 2 \mathrm{H}, \mathrm{Ar}-H), 6.62\left(\mathrm{~d}, 2 \mathrm{H},{ }^{3} J_{\mathrm{HH}}=8.5 \mathrm{~Hz}, \mathrm{Ar}-H\right), 6.40(\mathrm{~d}, 2 \mathrm{H}$, $\left.{ }^{3} J_{\mathrm{HH}}=8.5 \mathrm{~Hz}, \mathrm{Ar}-H\right), 3.90\left(\right.$ br q, 4H, $-\mathrm{OCH}_{2}-$ bound $\left.\mathrm{Et}_{2} \mathrm{O}\right), 1.91\left(\mathrm{~s}, 6 \mathrm{H}, \mathrm{Ar}-\mathrm{CH}_{3}\right), 1.06(\mathrm{t}, 6 \mathrm{H}$, ${ }^{3} J_{\mathrm{HH}}=7.1 \mathrm{~Hz},-\mathrm{OCH}_{2} \mathrm{CH}_{3}-$ bound $\left.\mathrm{Et}_{2} \mathrm{O}\right) \mathrm{ppm}$.

${ }^{13} \mathrm{C}\left\{{ }^{1} \mathrm{H}\right\}$ NMR $\left(126 \mathrm{MHz}, \mathrm{C}_{6} \mathrm{D}_{6}\right) \delta 148.6$ (Ar-C), 136.4 (Ar-C), 131.9 (Ar-C), 126.9 (Ar-C), 124.1 (Ar-C), $123.0(\mathrm{Ar}-\mathrm{C}), 85.0\left(-\mathrm{OC}\left(\mathrm{CF}_{3}\right)_{2}\right), 70.7\left(-\mathrm{OCH}_{2}-\right.$ bound $\left.\mathrm{Et}_{2} \mathrm{O}\right), 20.5\left(\mathrm{Ar}-\mathrm{CH}_{3}\right)$, $13.0\left(-\mathrm{OCH}_{2} \mathrm{CH}_{3}-\right.$ bound $\left.\mathrm{Et}_{2} \mathrm{O}\right)$ ppm.

${ }^{19} \mathrm{~F}$ NMR $\left(282 \mathrm{MHz}, \mathrm{C}_{6} \mathrm{D}_{6}\right) \delta-69.9\left(\mathrm{q}, 6 \mathrm{~F},{ }^{4} J_{\mathrm{FF}}=9.7 \mathrm{~Hz}\right),-73.5\left(\mathrm{q}, 6 \mathrm{~F},{ }^{4} J_{\mathrm{FF}}=9.7 \mathrm{~Hz}\right) \mathrm{ppm}$.

Anal. Calcd. for $\mathrm{C}_{24} \mathrm{H}_{22} \mathrm{Cl}_{2} \mathrm{~F}_{12} \mathrm{NNbO}_{3}(764.23 \mathrm{~g} / \mathrm{mol})$ : C, 37.72\%; H, 2.90\%; $\mathrm{N}, 1.83 \%$. Found: $\mathrm{C}, 36.97 \%, \mathrm{H}, 2.84 \%, \mathrm{~N}, 1.66 \%$ 


\section{NMR Characterization of $\left[\mathrm{CF}_{3}-\mathrm{ONO}\right] \mathrm{NbCl}_{2}\left(\mathrm{Et}_{2} \mathrm{O}\right)\left(3-\mathrm{Et}_{2} \mathrm{O}\right)$}

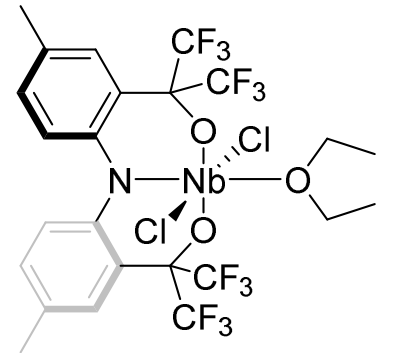

$3-\mathrm{Et}_{2} \mathrm{O}$

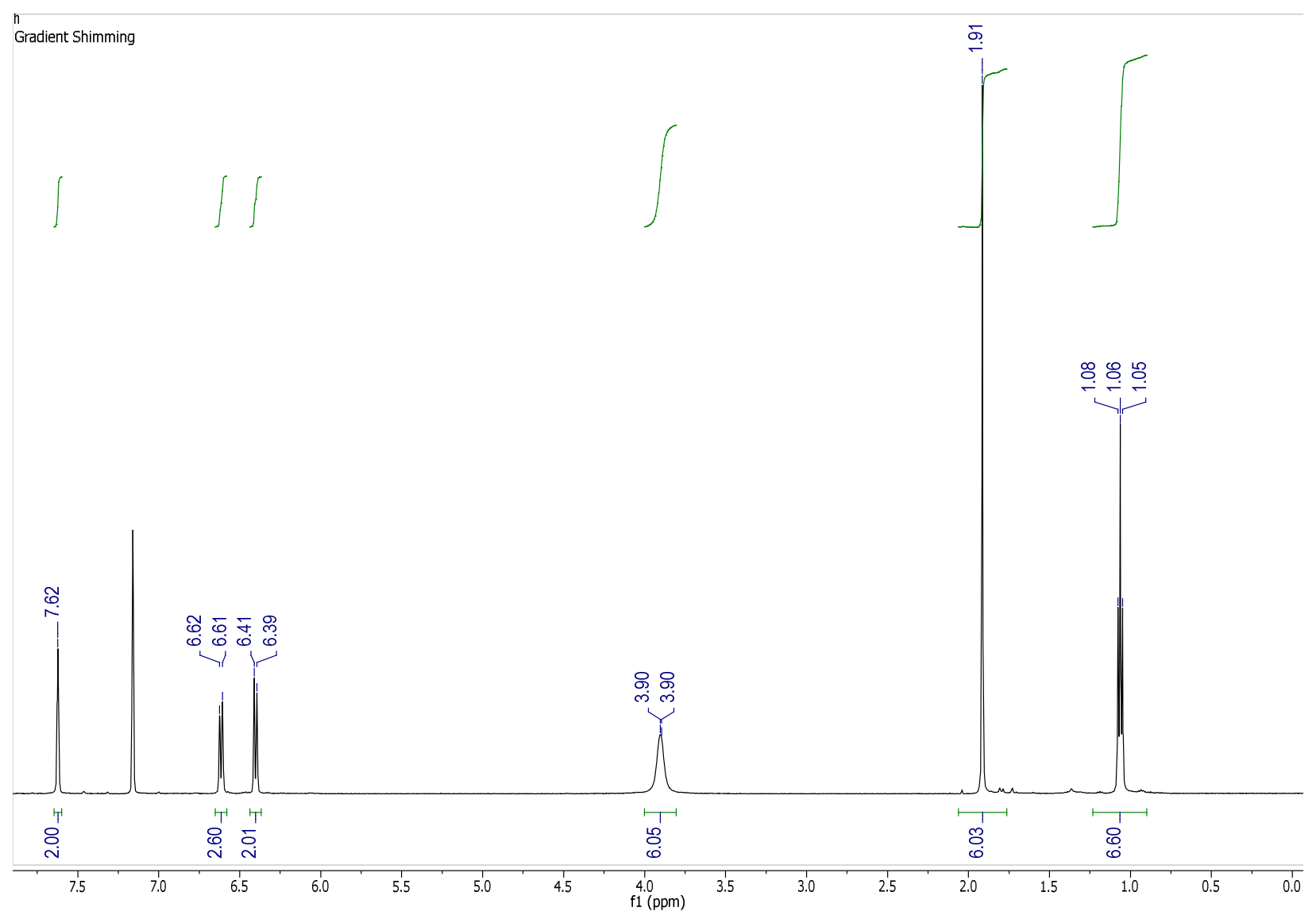

Figure S15. ${ }^{1} \mathrm{H}$ NMR spectrum of $\mathbf{3}-\mathbf{E t}_{2} \mathbf{O}\left(\mathrm{C}_{6} \mathrm{D}_{6}, 500 \mathrm{MHz}, 2{ }^{\circ} \mathrm{C}\right)$ 

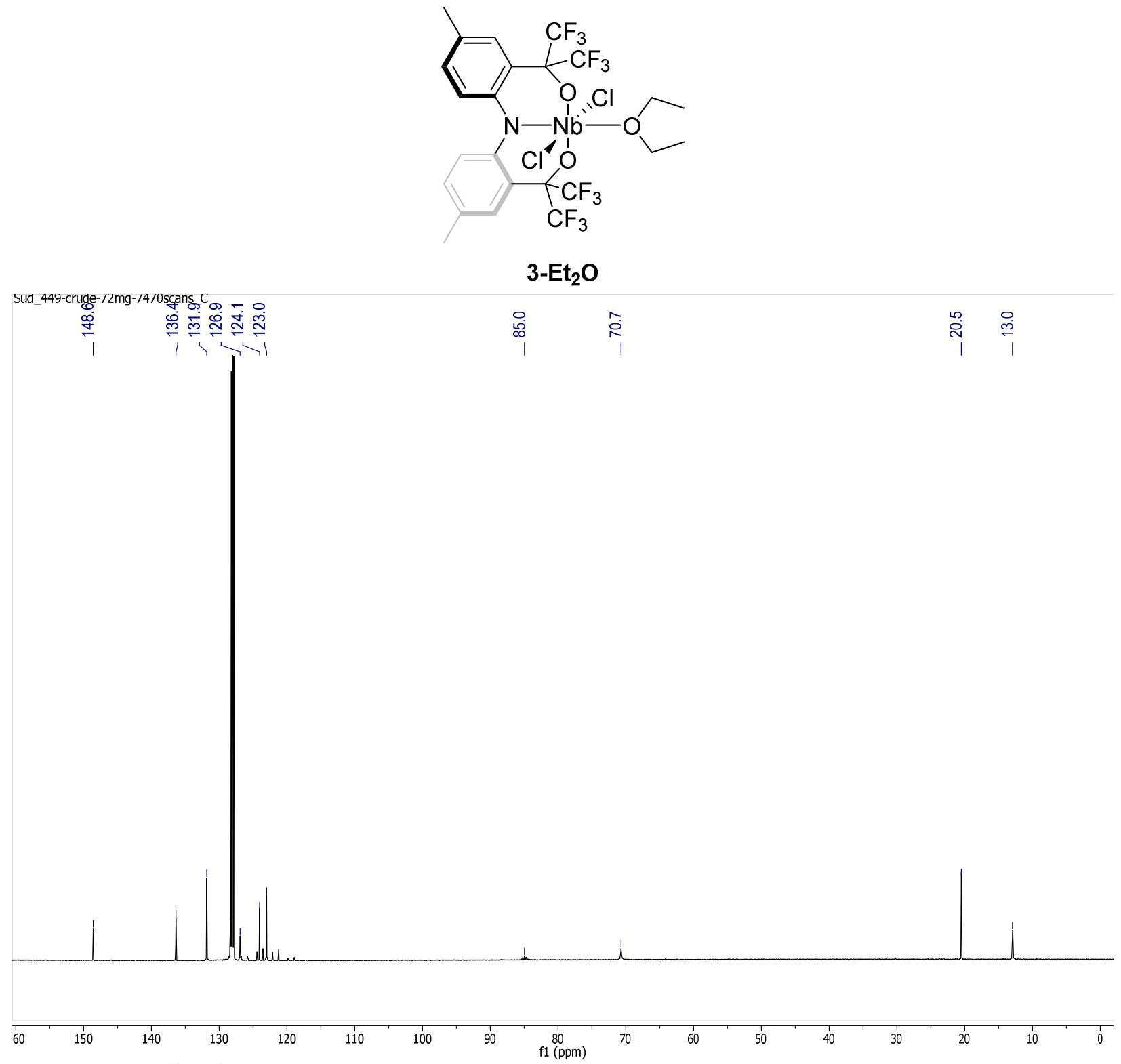

Figure S16. ${ }^{13} \mathrm{C}\left\{{ }^{1} \mathrm{H}\right\}$ NMR spectrum of 3-Et $\mathbf{t}_{2} \mathbf{O}\left(\mathrm{C}_{6} \mathrm{D}_{6}, 126 \mathrm{MHz}, 25{ }^{\circ} \mathrm{C}\right)$ 


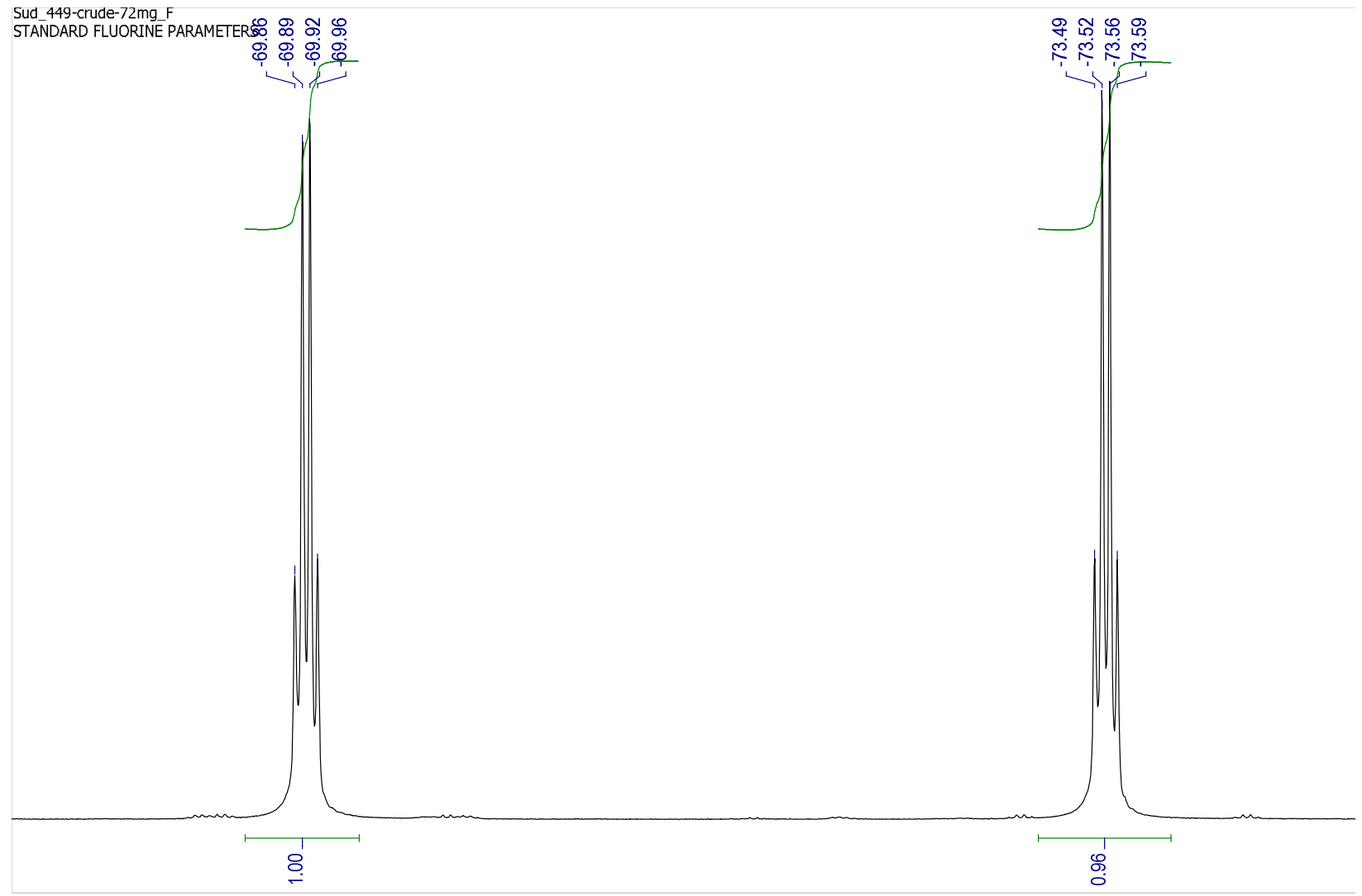

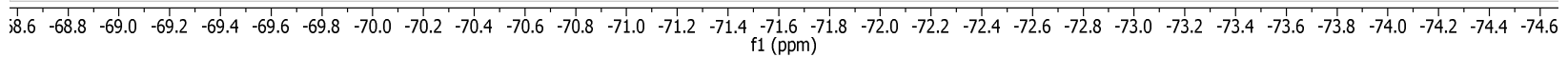
Figure S17. ${ }^{19} \mathrm{~F}$ NMR spectrum of 3-Et $\mathbf{2} \mathbf{O}\left(\mathrm{C}_{6} \mathrm{D}_{6}, 282 \mathrm{MHz}, 25{ }^{\circ} \mathrm{C}\right)$ 


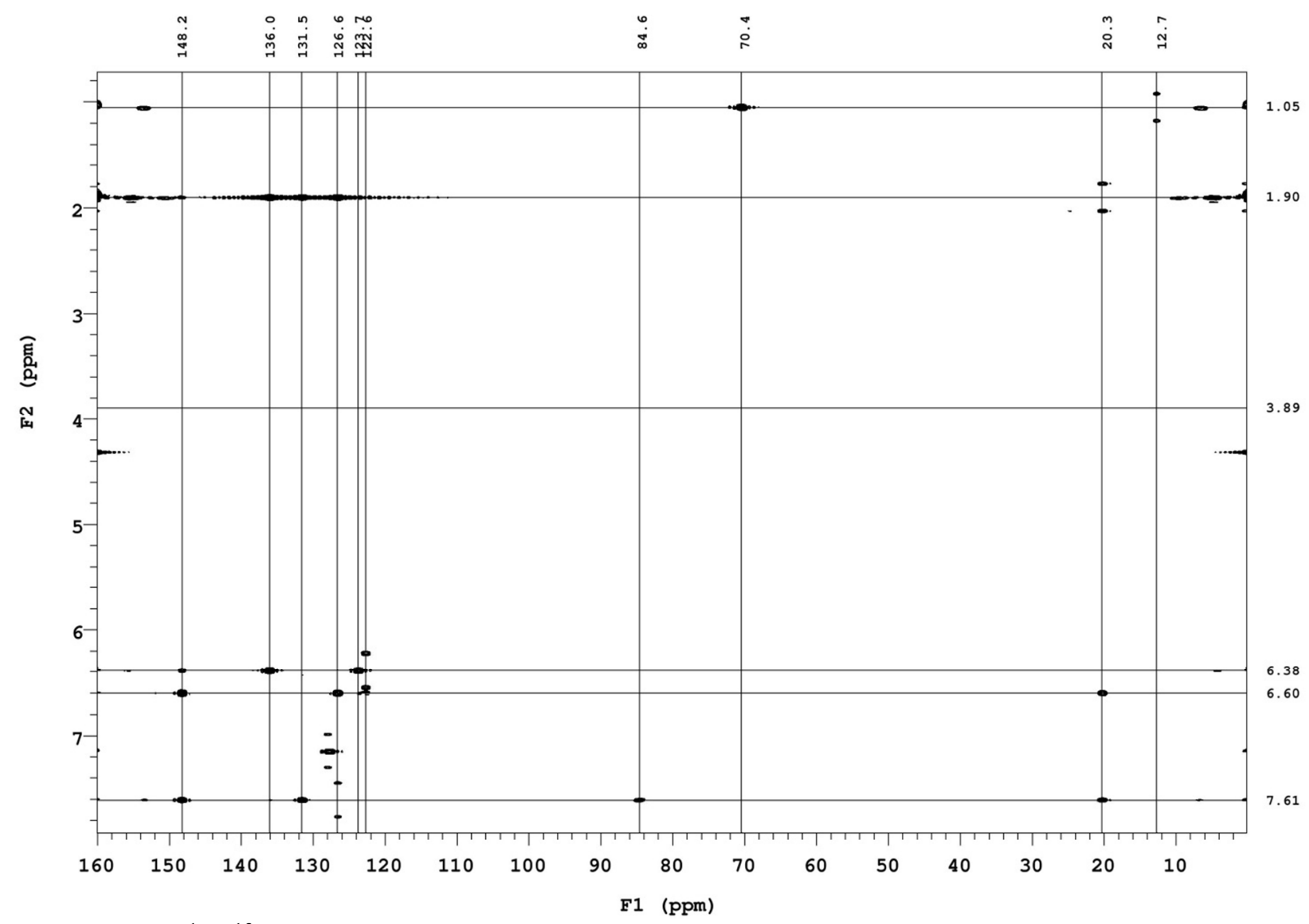

Figure S18. ${ }^{1} \mathrm{H}_{-}{ }^{13} \mathrm{C} g \mathrm{HMBC}$ spectrum of $\mathbf{3 - E t _ { 2 }} \mathbf{O}\left(\mathrm{C}_{6} \mathrm{D}_{6}, 500 \mathrm{MHz}, 25{ }^{\circ} \mathrm{C}\right)$ 


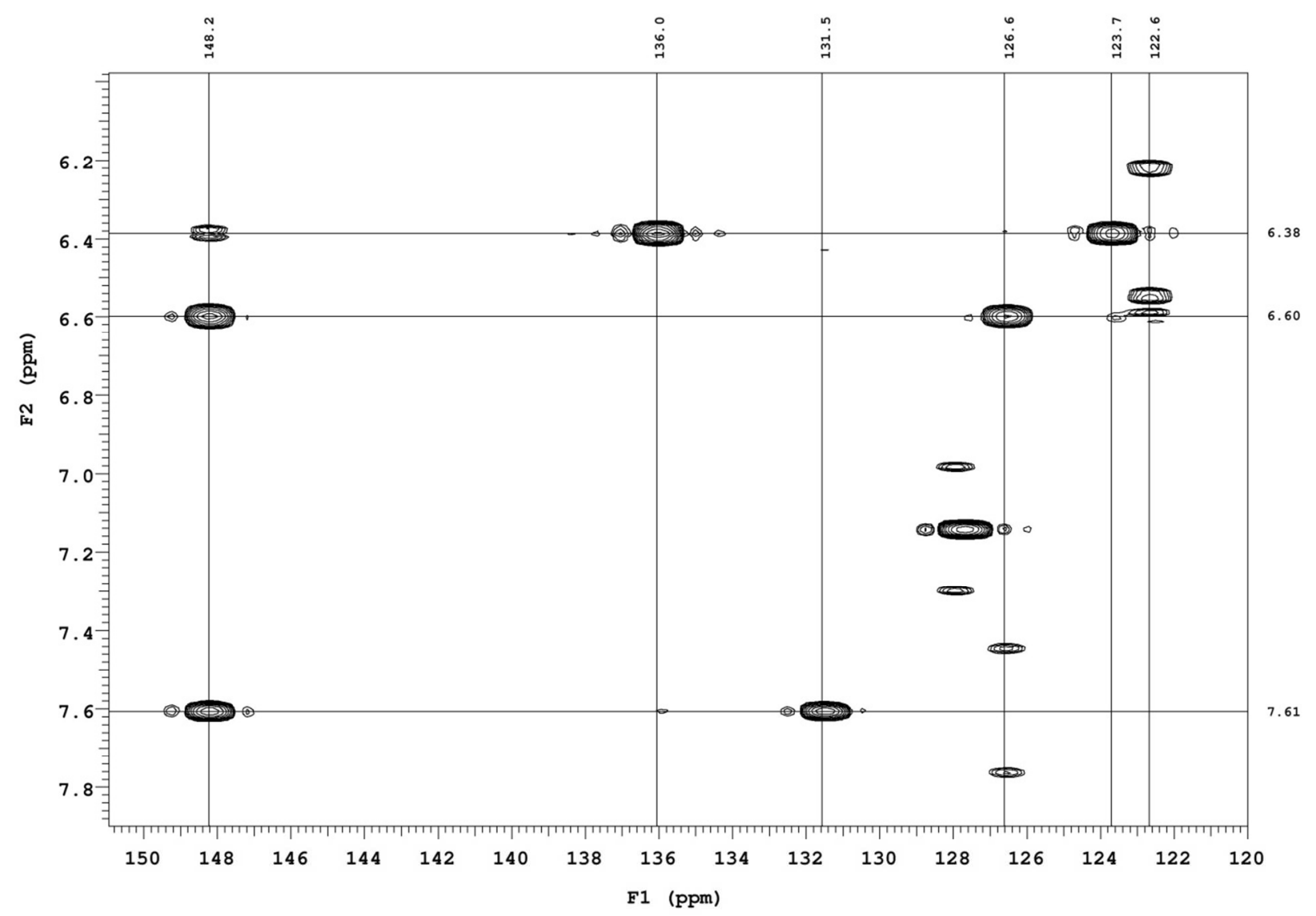

Figure S19. ${ }^{1} \mathrm{H}^{13} \mathrm{C} g \mathrm{HMBC}$ spectrum of $\mathbf{3 - E t _ { 2 }} \mathbf{O}-$ Expansion $\left(\mathrm{C}_{6} \mathrm{D}_{6}, 500 \mathrm{MHz}, 25{ }^{\circ} \mathrm{C}\right)$ 


\section{Synthesis of $\left[\mathrm{CF}_{3}-\mathrm{ONO}\right] \mathrm{NbCl}_{2}(\mathrm{~L})(3-\mathrm{L})$}

Complexes 3-L, variants of $\mathbf{3}-\mathbf{E t}_{\mathbf{2}} \mathbf{O}$, are obtained either through displacement of the bound molecule of diethyl ether in $\mathbf{3}-\mathbf{E t}_{\mathbf{2}} \mathbf{O}$ by an L-type ligand or the direct addition of an appropriate L-type ligand to 2.

Method 1: To $0.015 \mathrm{~g}$ of $\mathbf{3}-\mathbf{E t}_{\mathbf{2}} \mathbf{O}$ dissolved in $0.7 \mathrm{~mL} \mathrm{C}_{6} \mathrm{D}_{6}$, excess of an L-type ligand (ACN, THF, DME, py, and $\mathrm{O}=\mathrm{PPh}_{3}$ ) was added. The solution was thoroughly mixed with a Pasteur pipette and transferred to an NMR tube for characterization.

Method 2: To $0.020 \mathrm{~g}$ of 2 dissolved in $\mathrm{C}_{6} \mathrm{H}_{6}$, a four-fold excess of an L-type ligand (ACN, py, $\mathrm{O}=\mathrm{PPh}_{3}$ ) was added. Stirring overnight leads to a color change from brown to maroon / purple. The solution was filtered through a celite pad and all volatiles were removed in vacuo to afford a maroon / purple solid. The solid residue was dissolved in $\mathrm{C}_{6} \mathrm{D}_{6}$ for comparison to Method 1 . The yield for the reactions was not quantified. 


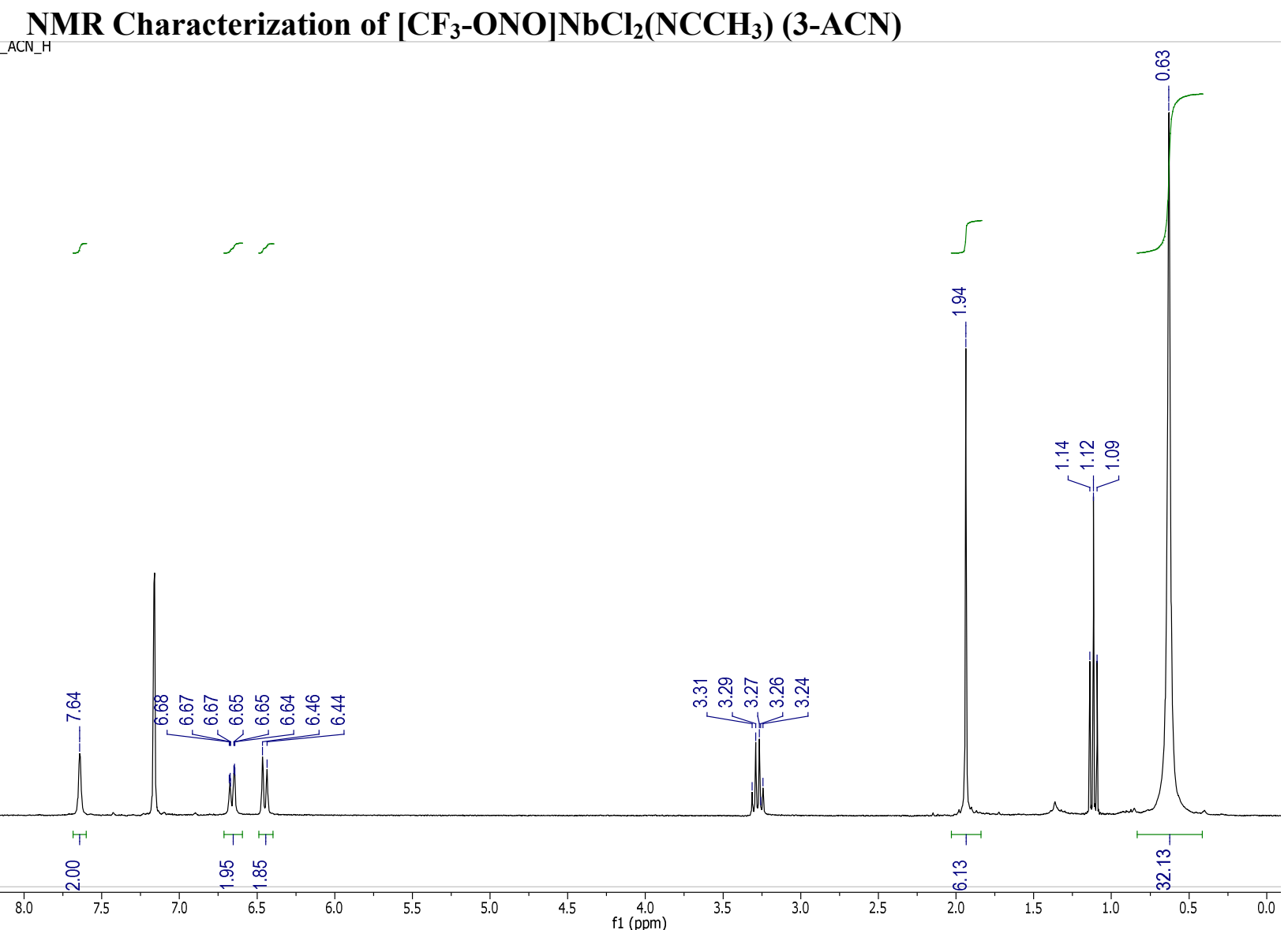

Figure S20. ${ }^{1} \mathrm{H}$ NMR spectrum of 3-ACN $\left(\mathrm{C}_{6} \mathrm{D}_{6}, 300 \mathrm{MHz}\right)$ - Method 1 (Presence of free $\mathrm{Et}_{2} \mathrm{O}$ and excess acetonitrile) 


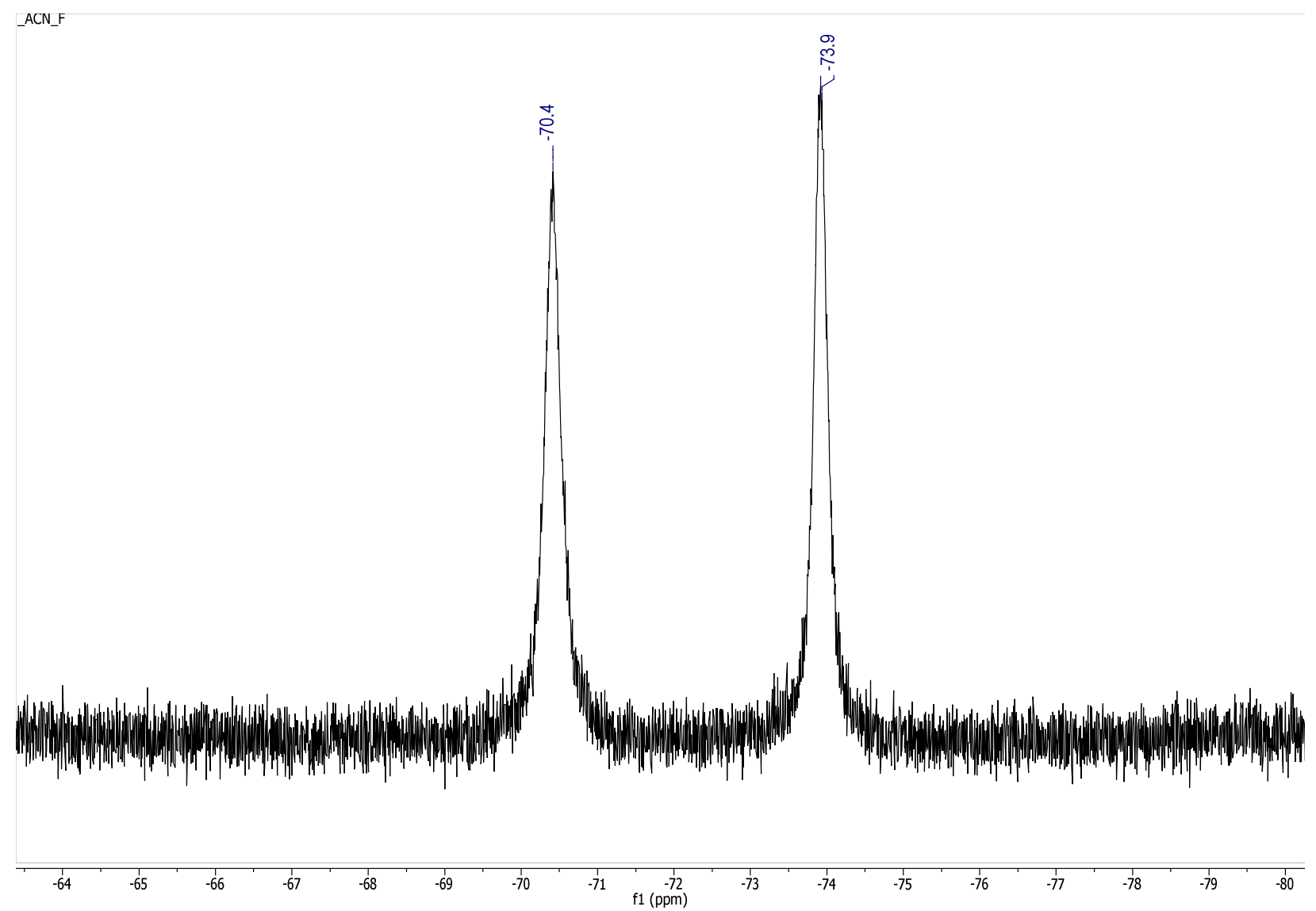

Figure S21. ${ }^{19} \mathrm{~F}$ NMR spectrum of 3-ACN $\left(\mathrm{C}_{6} \mathrm{D}_{6}, 282 \mathrm{MHz}\right)-$ Method 1 


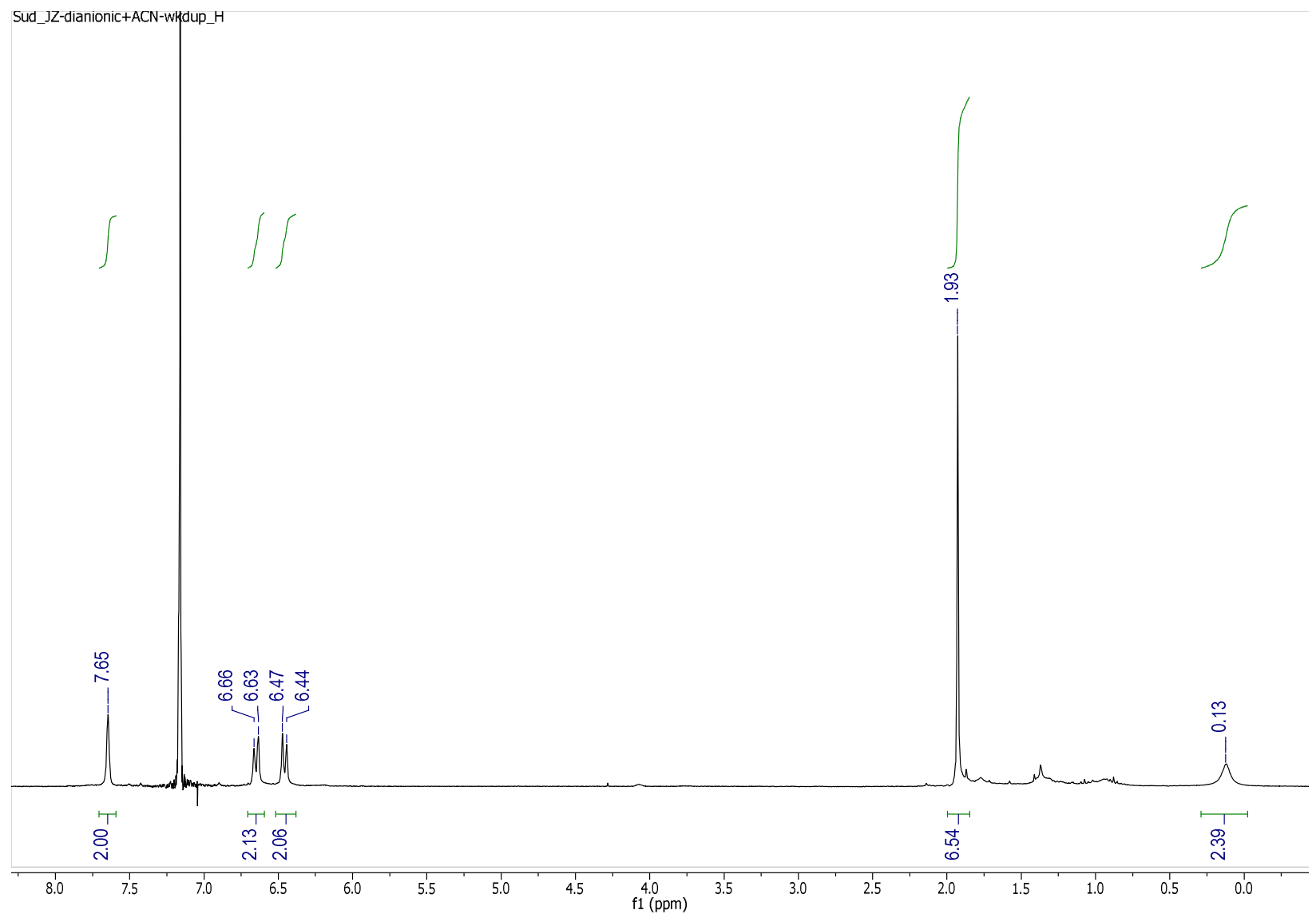

Figure S22. ${ }^{1} \mathrm{H}$ NMR spectrum of 3-ACN $\left(\mathrm{C}_{6} \mathrm{D}_{6}, 300 \mathrm{MHz}\right)-$ Method 2 


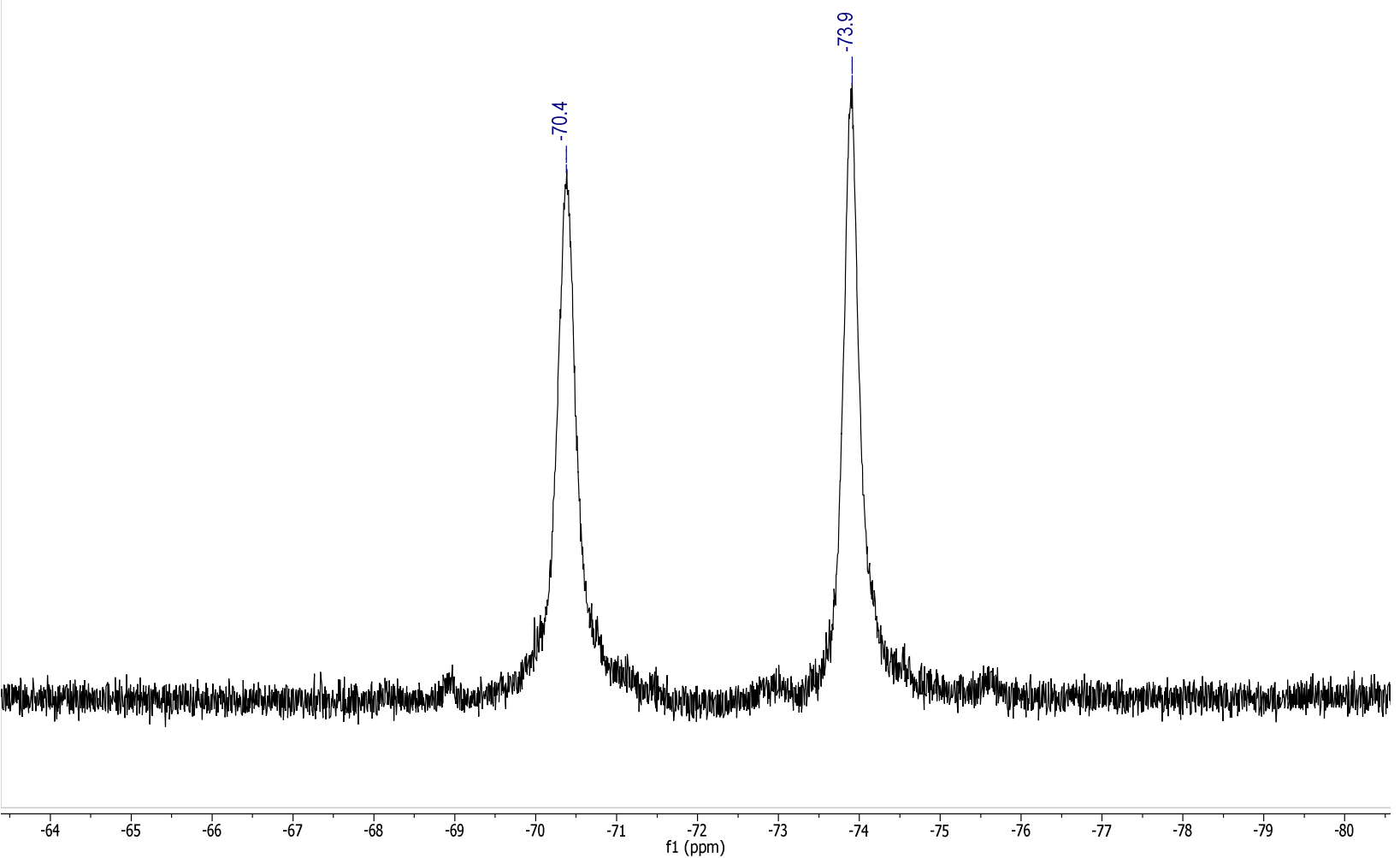

Figure S23. ${ }^{19}$ F NMR spectrum of 3-ACN $\left(\mathrm{C}_{6} \mathrm{D}_{6}, 282 \mathrm{MHz}\right)-$ Method 2 
NMR Characterization of $\left[\mathrm{CF}_{3}-\mathrm{ONO}\right] \mathrm{NbCl}_{2}$ (py) (3-py)

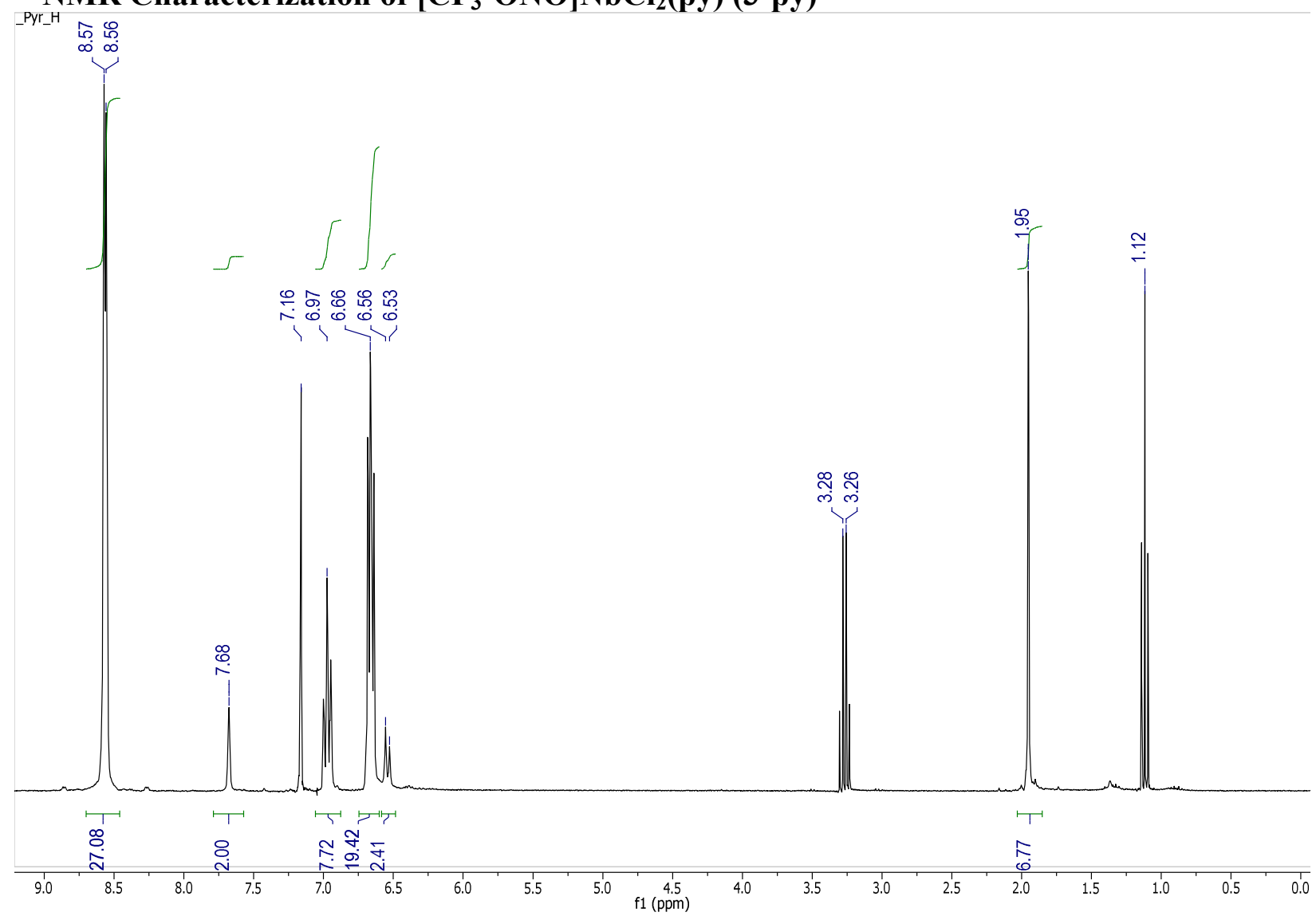

Figure S24. ${ }^{1} \mathrm{H}$ NMR spectrum of 3-py $\left(\mathrm{C}_{6} \mathrm{D}_{6}, 300 \mathrm{MHz}\right.$ ) - Method 1 (Presence of free $\mathrm{Et}_{2} \mathrm{O}$ and excess pyridine) 


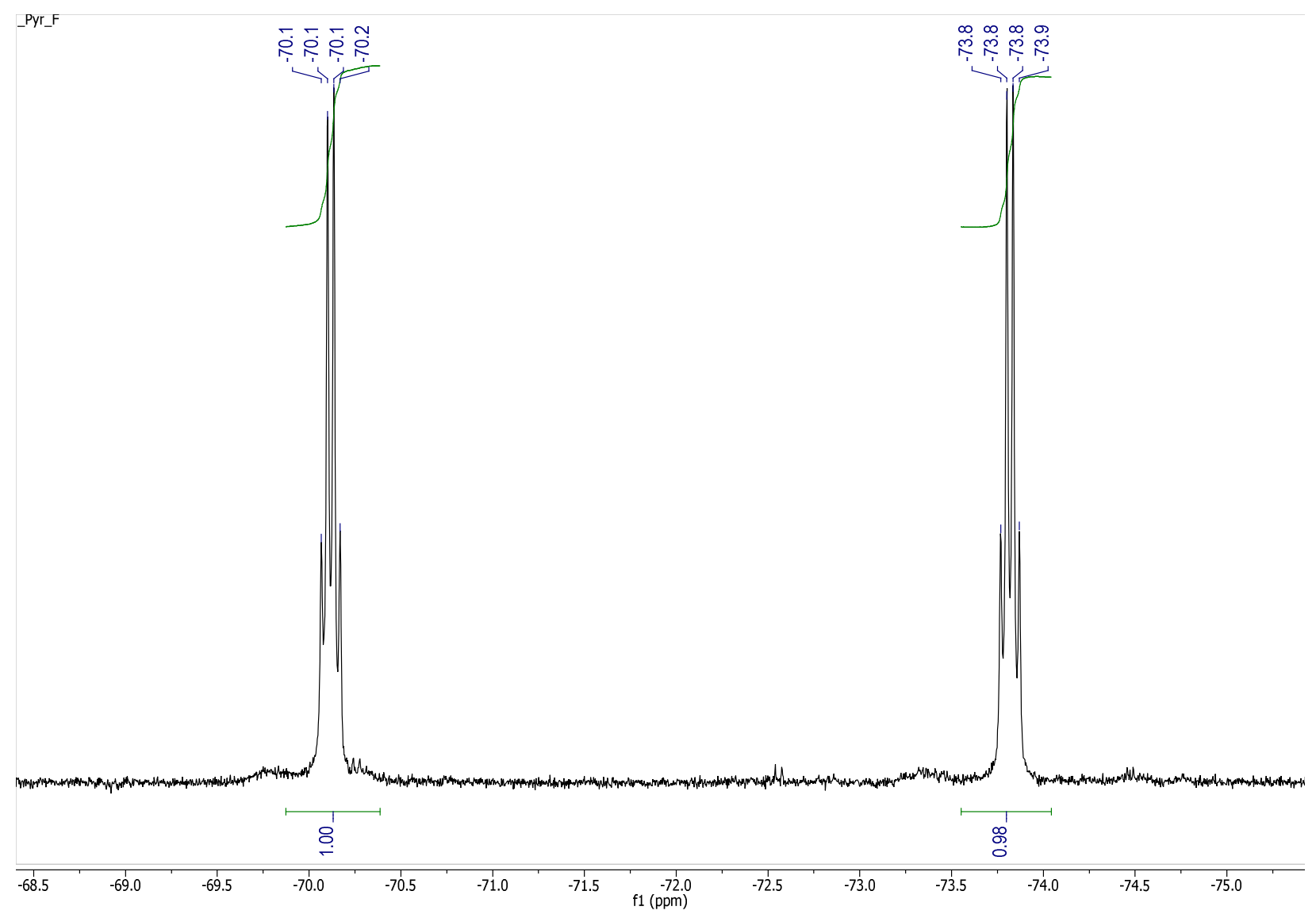

Figure S25. ${ }^{19} \mathrm{~F}$ NMR spectrum of 3-py $\left(\mathrm{C}_{6} \mathrm{D}_{6}, 282 \mathrm{MHz}\right)-$ Method 1 


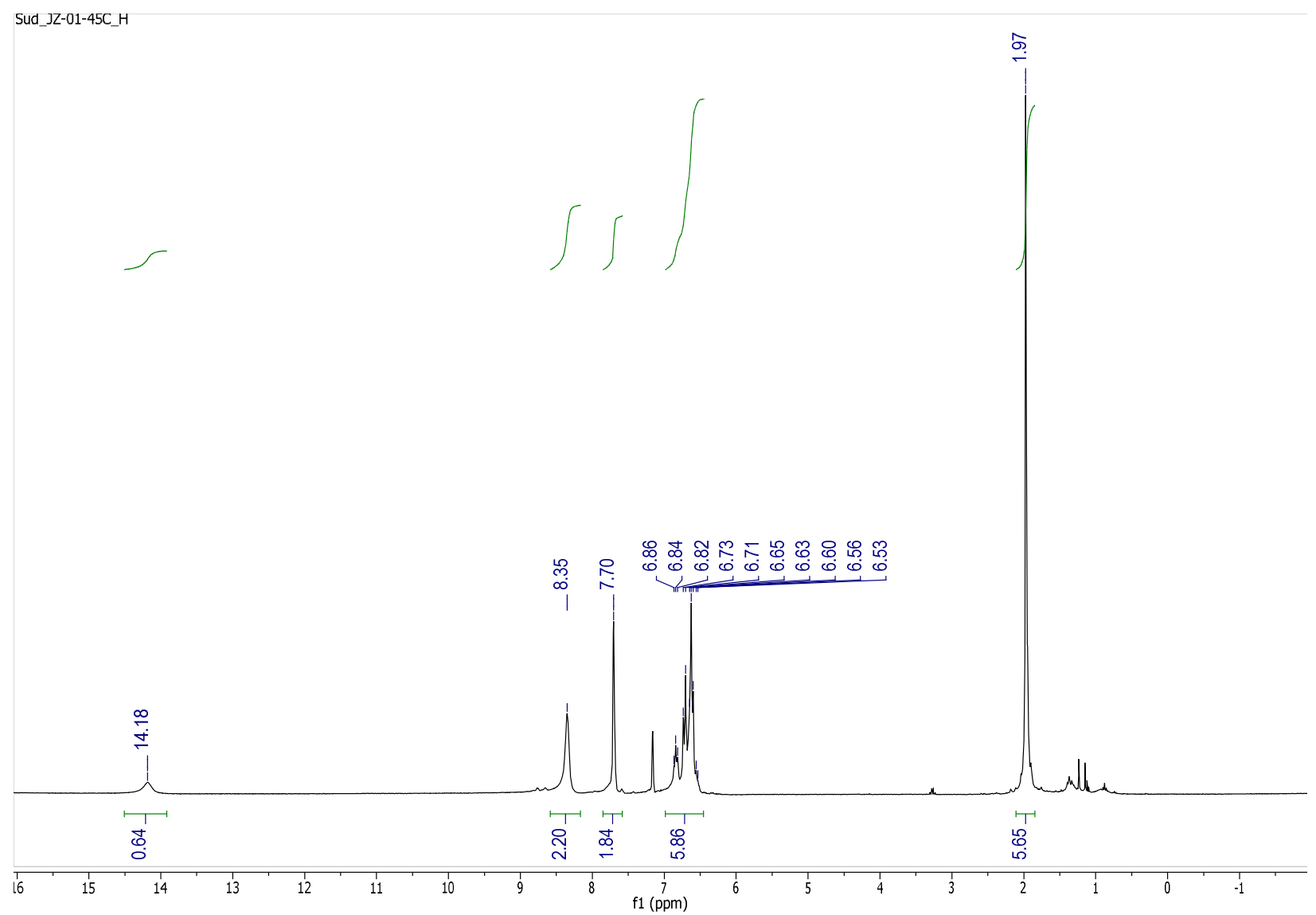

Figure S26. ${ }^{1} \mathrm{H}$ NMR spectrum of 3-py $\left(\mathrm{C}_{6} \mathrm{D}_{6}, 300 \mathrm{MHz}\right)-$ Method 2 


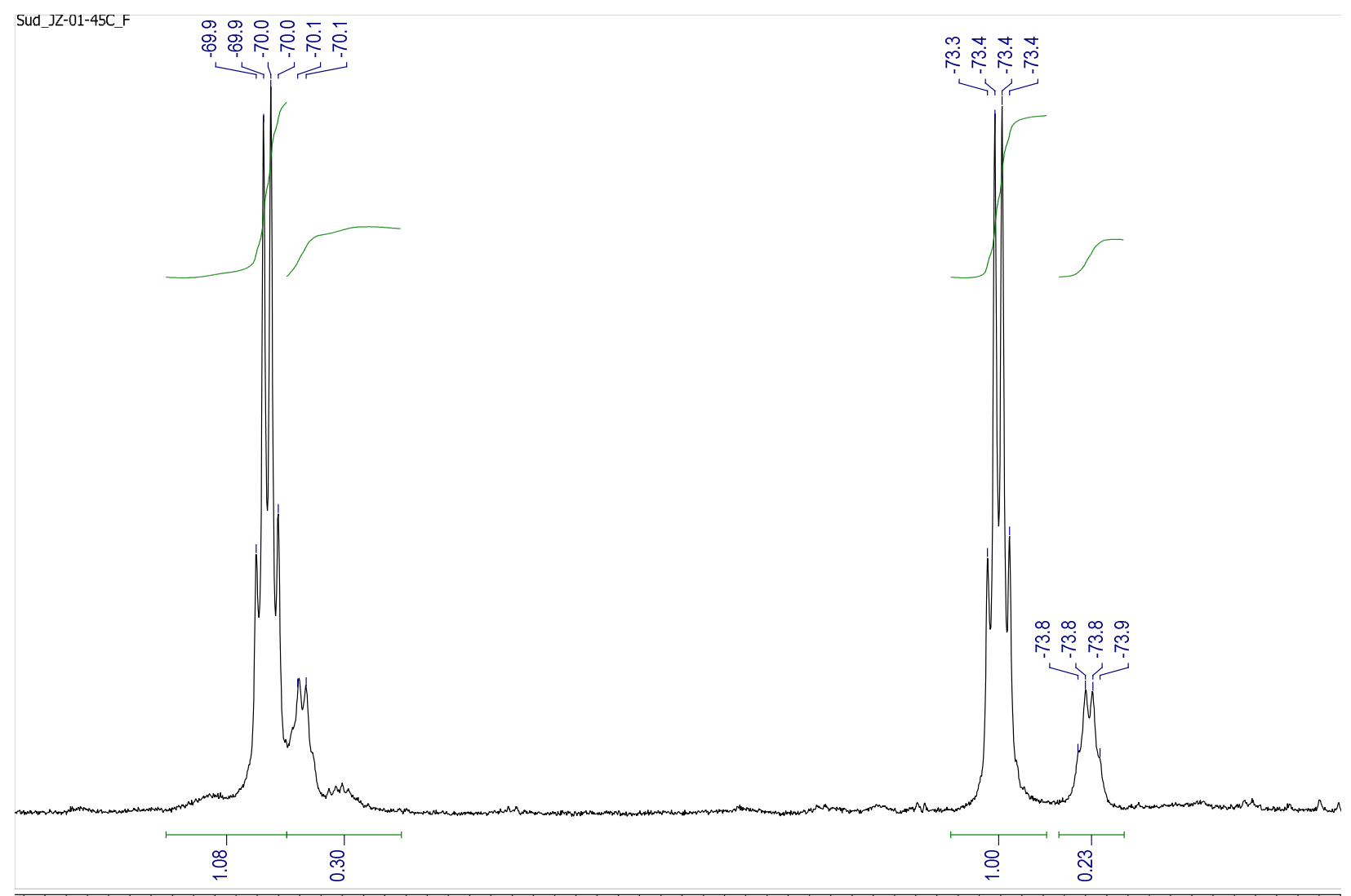

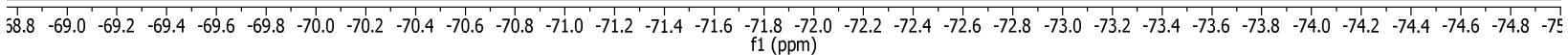

Figure S27. ${ }^{19}$ F NMR spectrum of 3-py $\left(\mathrm{C}_{6} \mathrm{D}_{6}, 282 \mathrm{MHz}\right)$ - Method 2

Adding pyridine to $\left[\mathrm{CF}_{3}-\mathrm{ONHO}\right] \mathrm{NbCl}_{3}$ generates the complex salt $\left\{\left[\mathrm{CF}_{3}-\mathrm{ONO}\right] \mathrm{NbCl}_{3}\right\}\{\mathrm{py} \bullet \mathrm{H}\}$ (major) in addition to the desired $\left[\mathrm{CF}_{3}-\mathrm{ONO}\right] \mathrm{NbCl}_{2}$ (py) (minor). Salt formation occurs for any L-type ligand that can also serve as an external base. 
$\mathrm{NMR}$ characterization of $\left[\mathrm{CF}_{3}-\mathrm{ONO}\right] \mathrm{NbCl}_{2}\left(\mathrm{O}=\mathrm{PPh}_{3}\right)\left(3-\mathrm{O}=\mathrm{PPh}_{3}\right)$

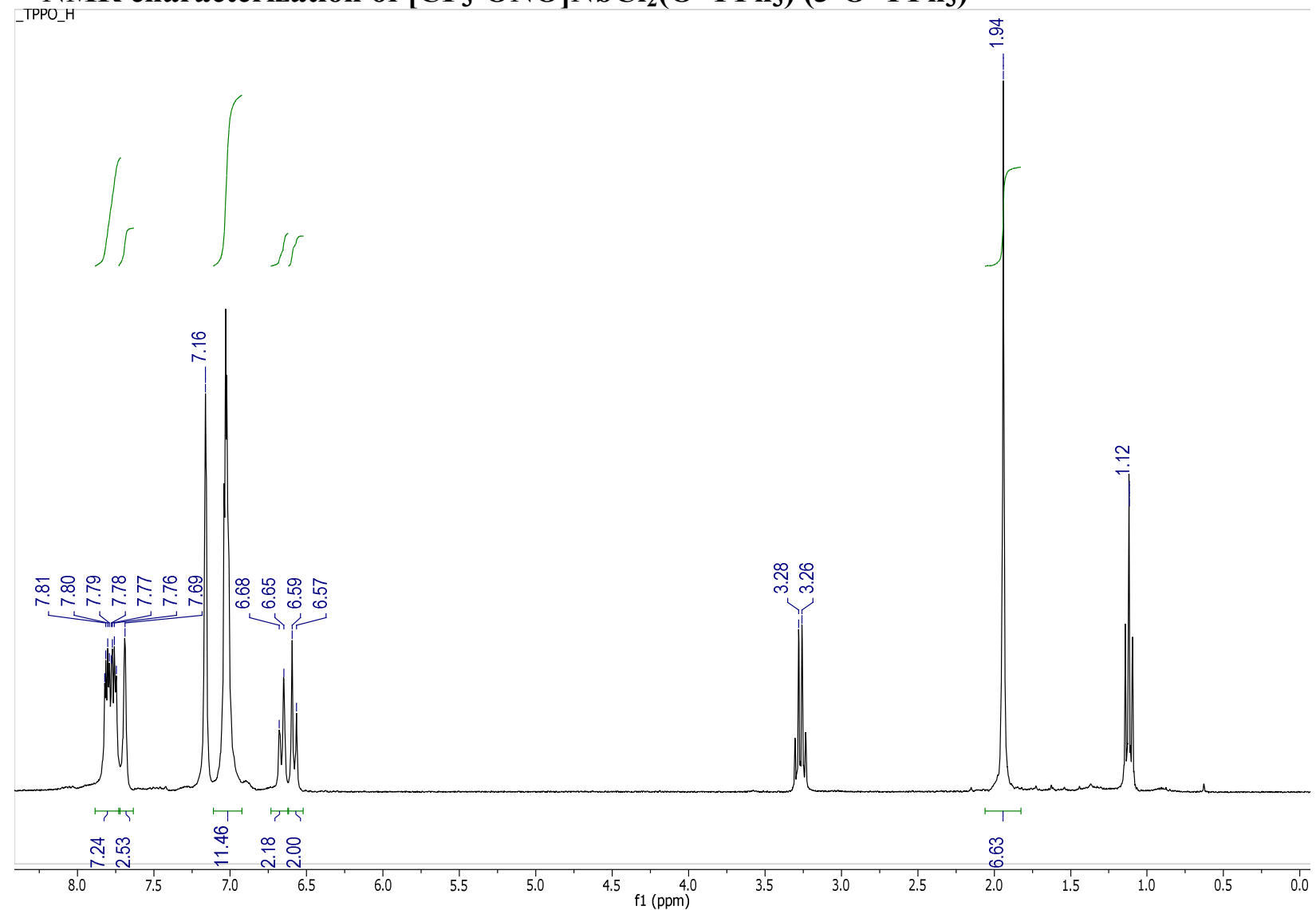

Figure S28. ${ }^{1} \mathrm{H}$ NMR spectrum of $\mathbf{3 - O}=\mathbf{P P h}_{\mathbf{3}}\left(\mathrm{C}_{6} \mathrm{D}_{6}, 300 \mathrm{MHz}\right.$ ) - Method 1 (Presence of free $\mathrm{Et}_{2} \mathrm{O}$ and excess $\mathrm{O}=\mathrm{PPh}_{3}$ ) 


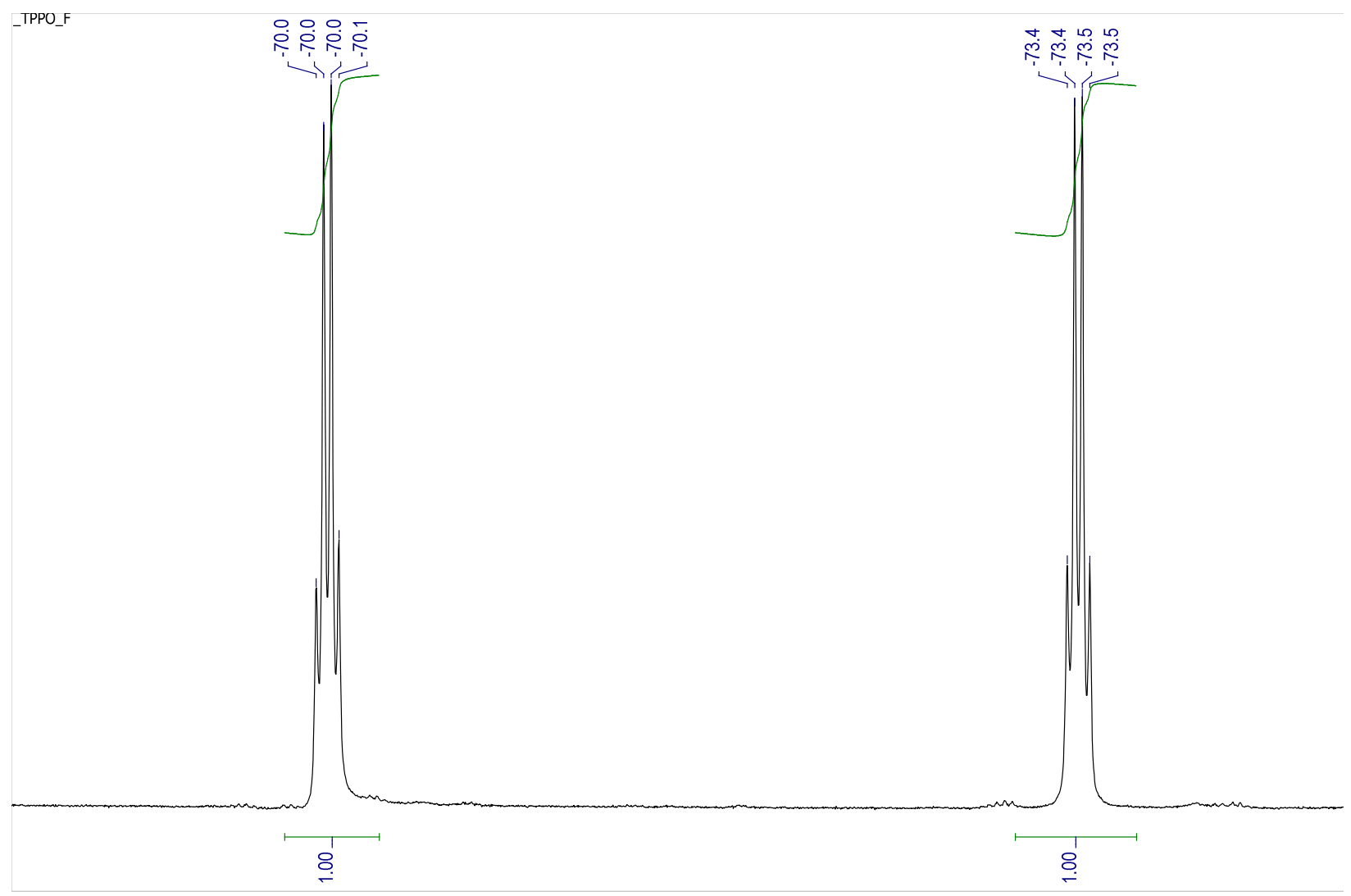

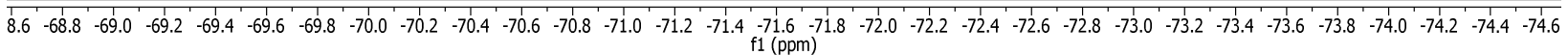
Figure S29. ${ }^{19} \mathrm{~F}$ NMR spectrum of 3-O=PPh $\left(\mathrm{C}_{6} \mathrm{D}_{6}, 282 \mathrm{MHz}\right)-$ Method 1 


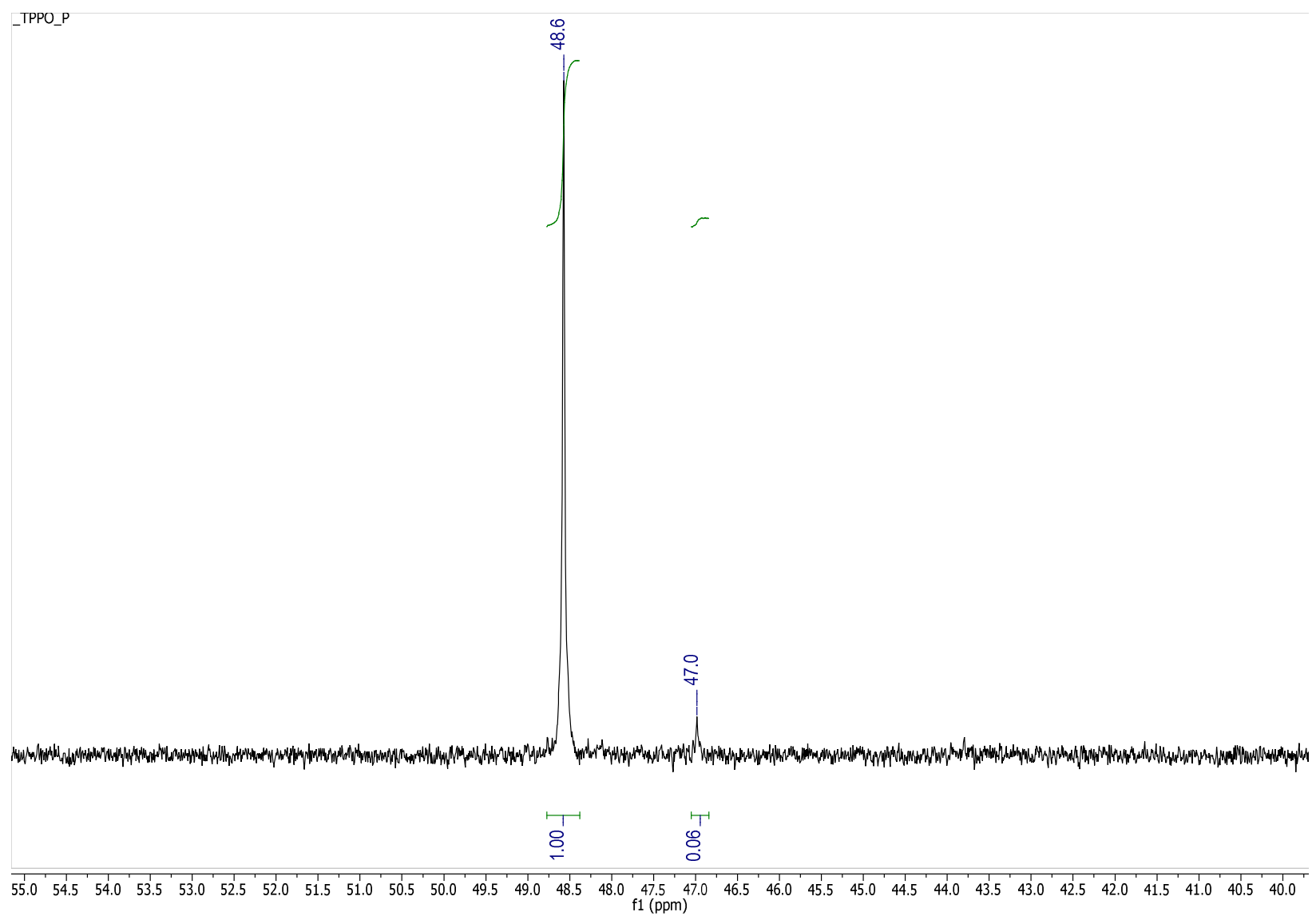

Figure S30. ${ }^{31} \mathrm{P}\left\{{ }^{1} \mathrm{H}\right\}$ NMR spectrum of 3-O=PPh $\left(\mathrm{C}_{6} \mathrm{D}_{6}, 121 \mathrm{MHz}\right)$ - Method 1 


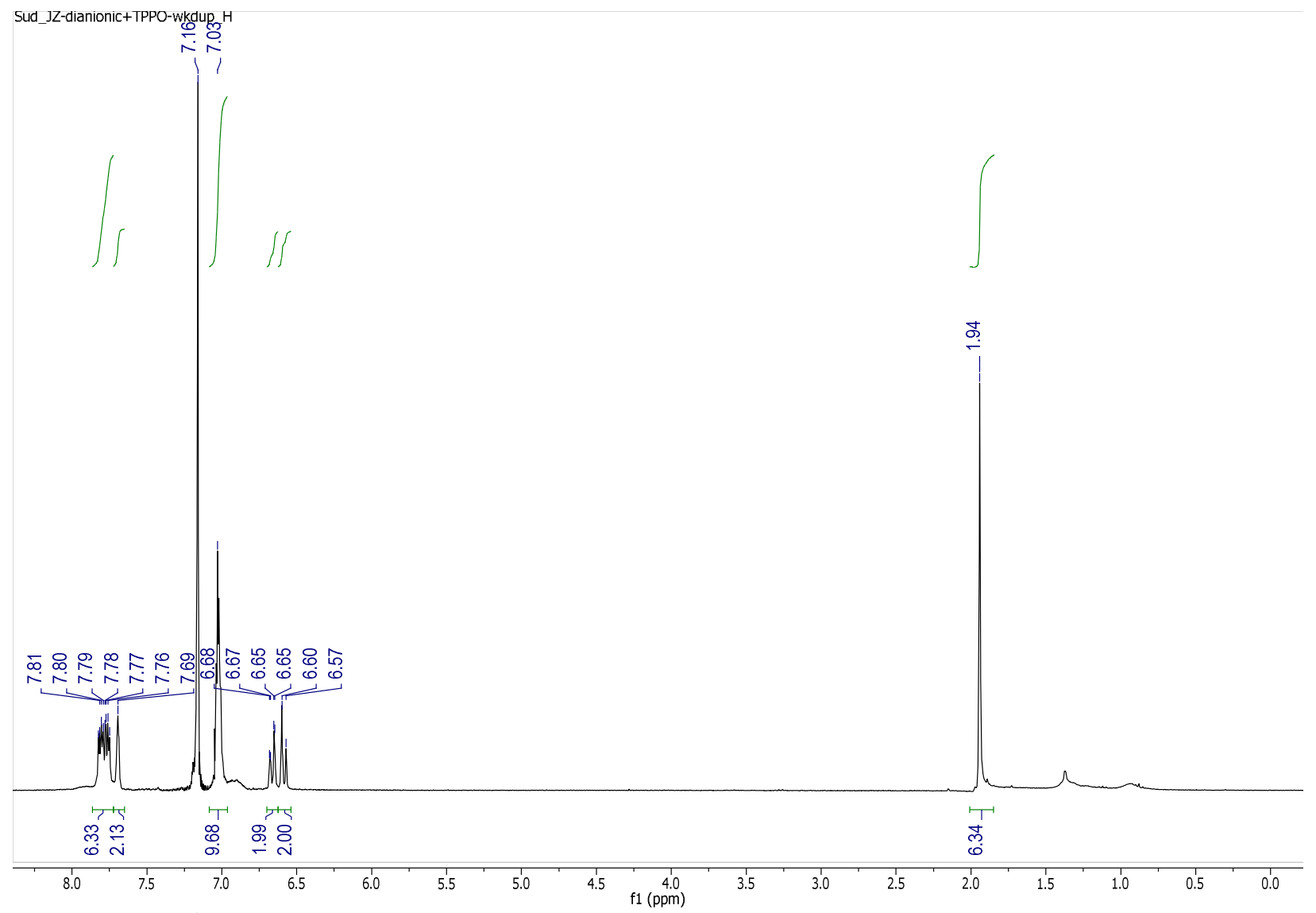

Figure S31. ${ }^{1} \mathrm{H}$ NMR spectrum of $\mathbf{3 - O}=\mathbf{P P h}_{3}\left(\mathrm{C}_{6} \mathrm{D}_{6}, 300 \mathrm{MHz}\right)-$ Method 2 


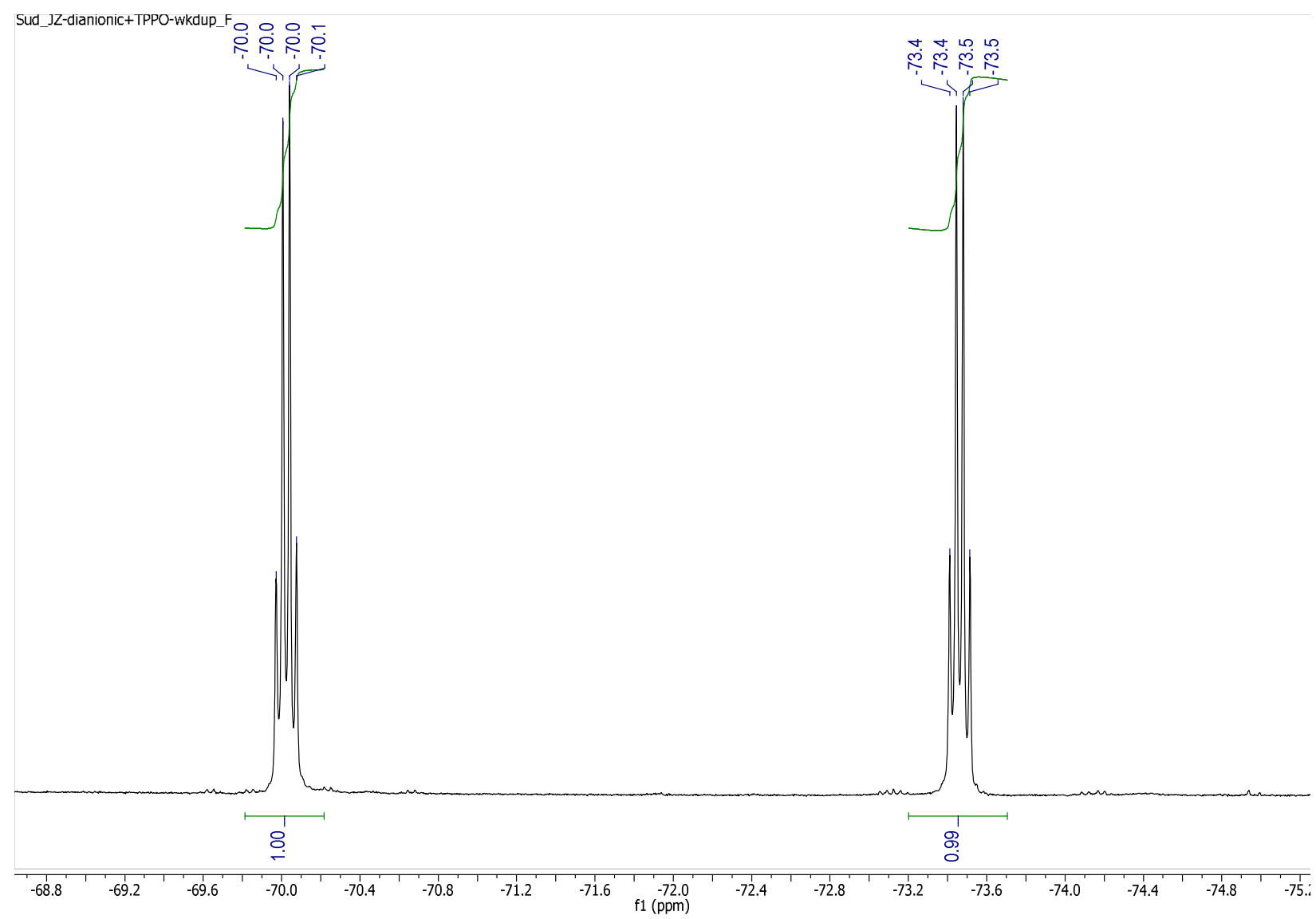

Figure S32. ${ }^{19} \mathrm{~F}$ NMR spectrum of $\mathbf{3 - O}=\mathbf{P P h}_{3}\left(\mathrm{C}_{6} \mathrm{D}_{6}, 282 \mathrm{MHz}\right)-$ Method 2 


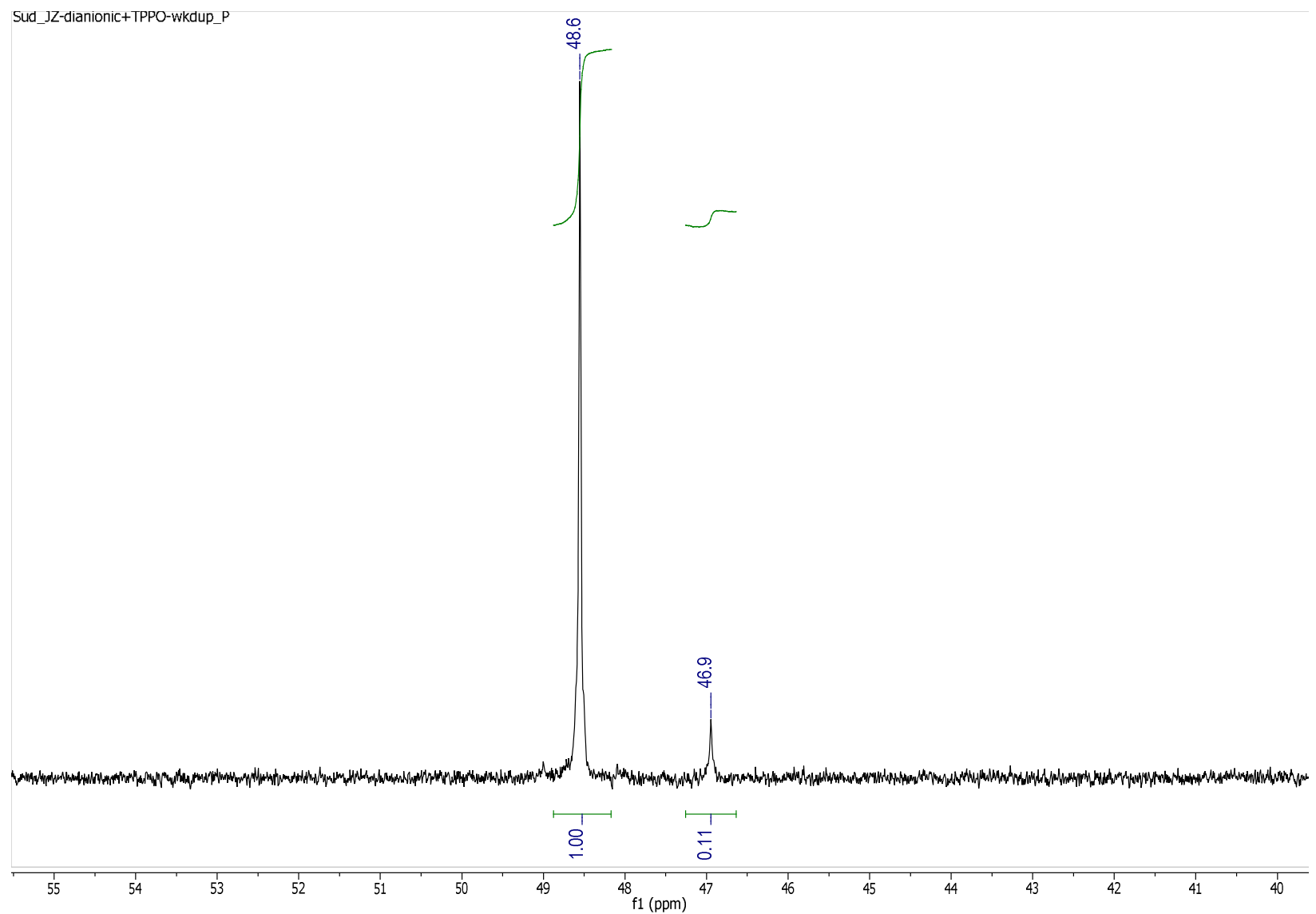

Figure S33. ${ }^{31} \mathrm{P}\left\{{ }^{1} \mathrm{H}\right\} \mathrm{NMR}$ spectrum of $\mathbf{3 - O}=\mathbf{P P h}_{3}\left(\mathrm{C}_{6} \mathrm{D}_{6}, 121 \mathrm{MHz}\right)-$ Method 2 


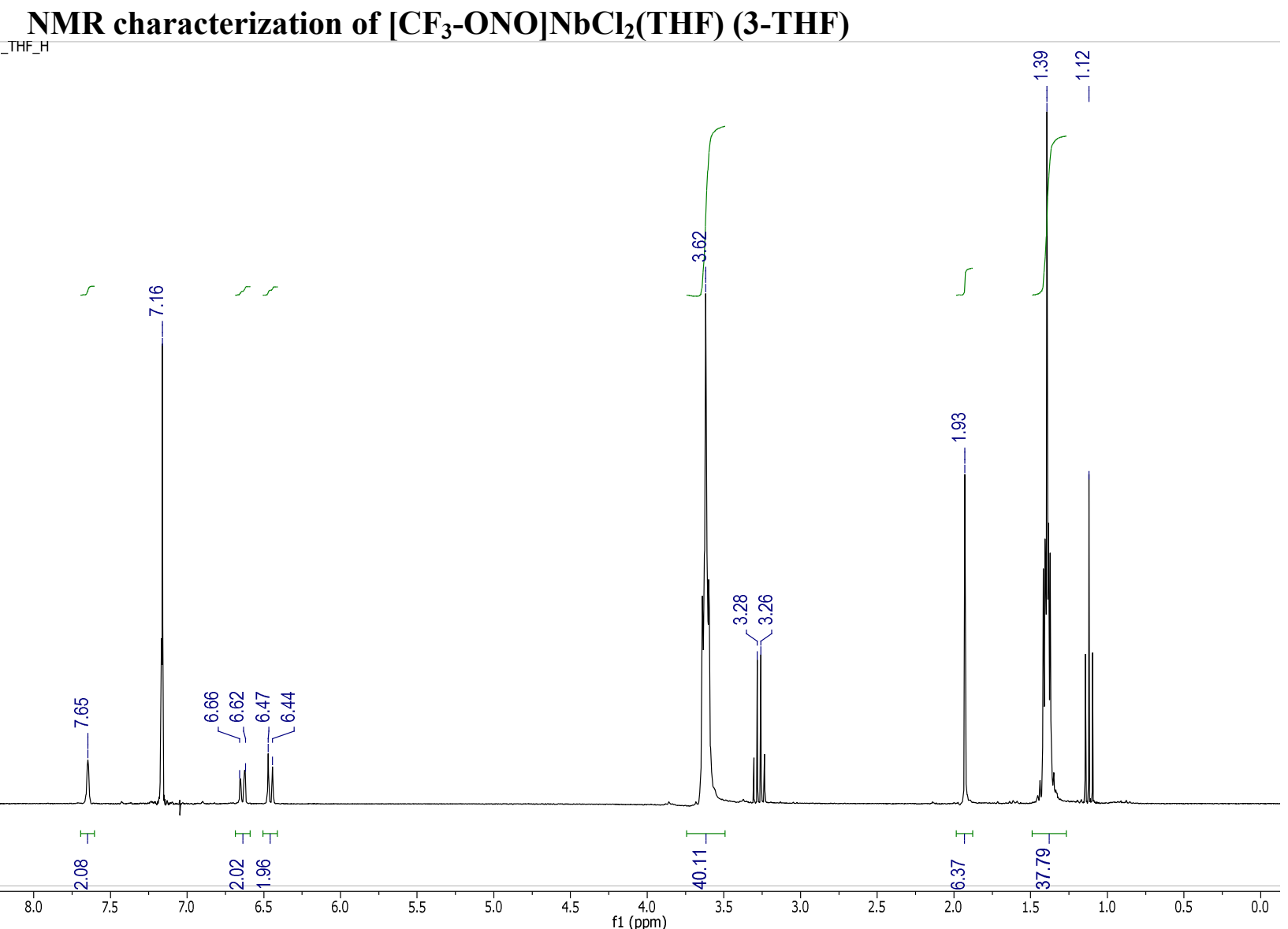

Figure S34. ${ }^{1} \mathrm{H}$ NMR spectrum of 3-THF $\left(\mathrm{C}_{6} \mathrm{D}_{6}, 300 \mathrm{MHz}\right)$ - Method 1 (Presence of free Et2O and excess THF) 


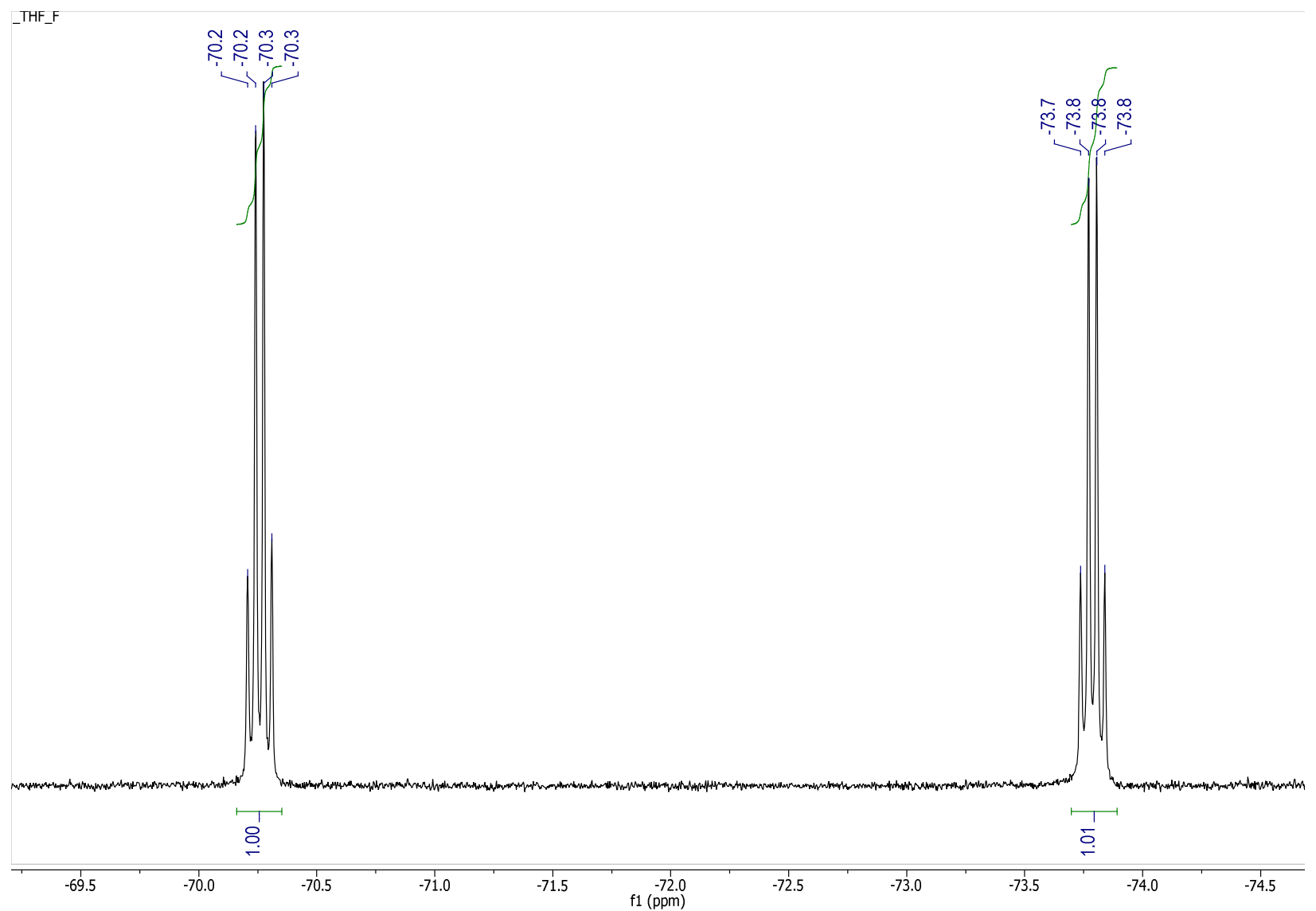

Figure S35. ${ }^{19} \mathrm{~F}$ NMR spectrum of 3-THF $\left(\mathrm{C}_{6} \mathrm{D}_{6}, 282 \mathrm{MHz}\right)-$ Method 1 


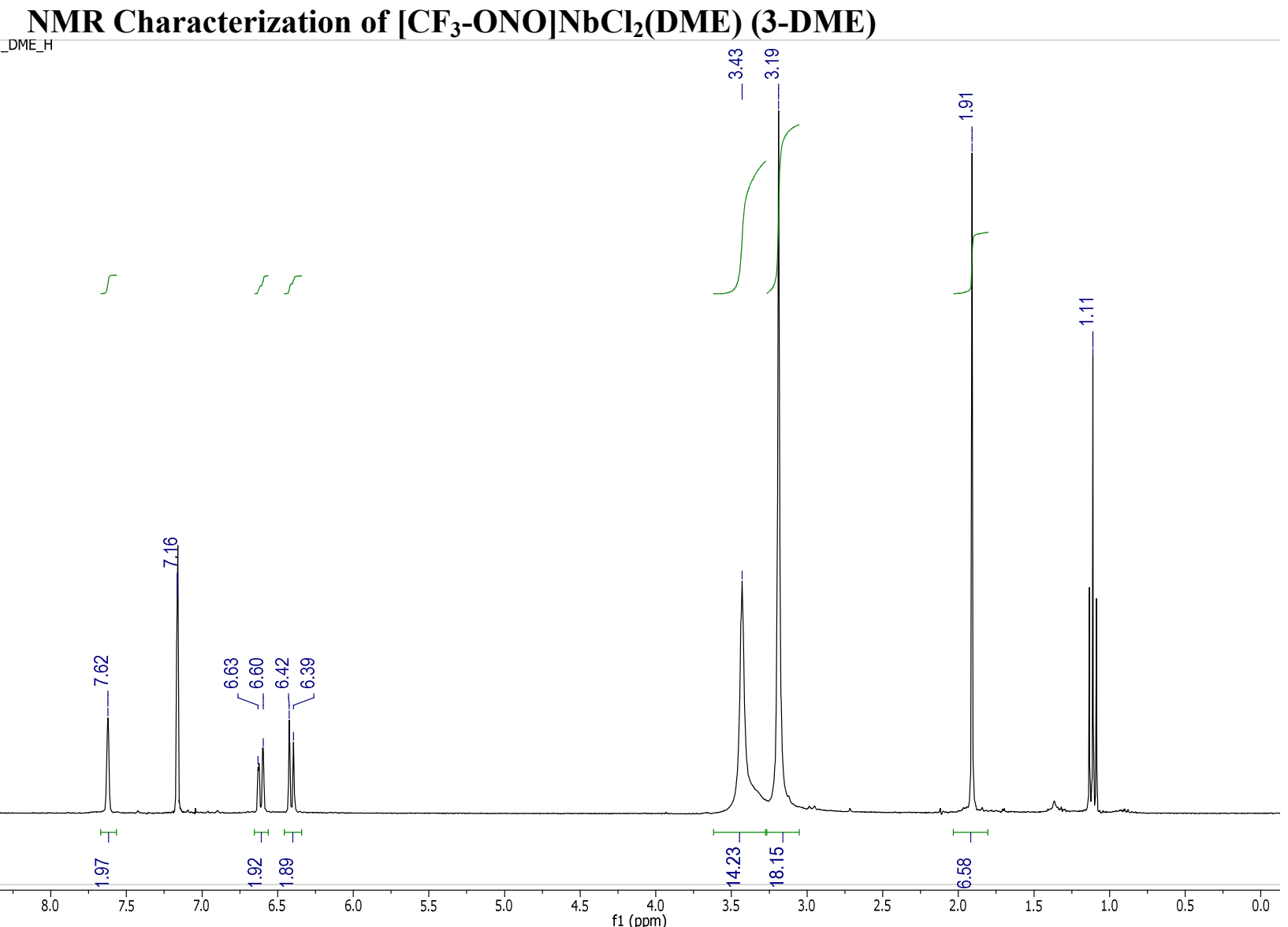

Figure S36. ${ }^{1} \mathrm{H}$ NMR spectrum of 3-DME $\left(\mathrm{C}_{6} \mathrm{D}_{6}, 300 \mathrm{MHz}\right)$ - Method 1 (Presence of free $\mathrm{Et}_{2} \mathrm{O}$ and excess DME) 


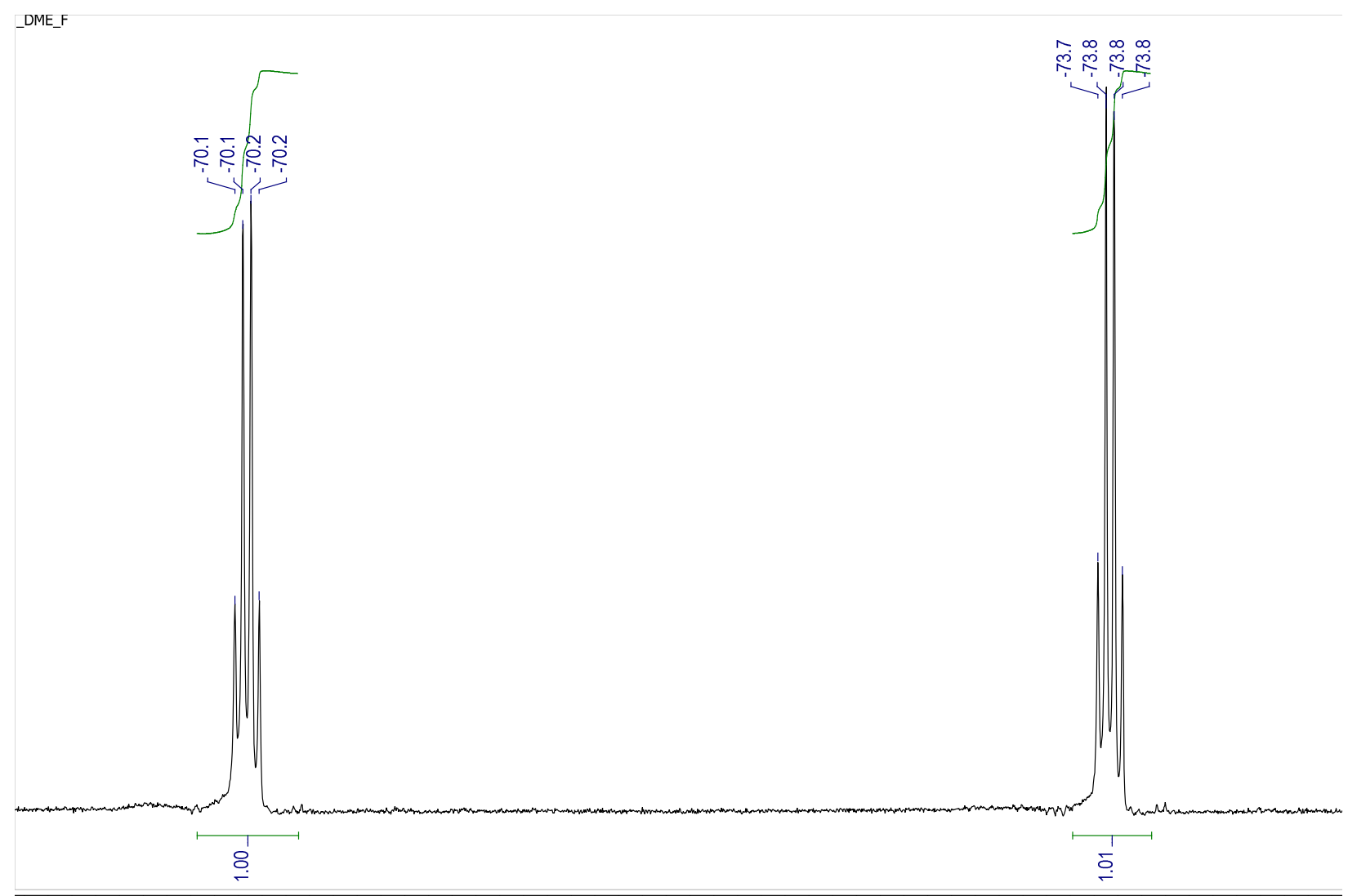

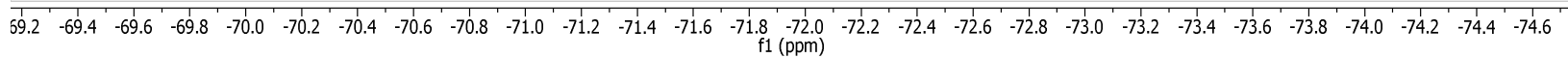
Figure S37. ${ }^{19} \mathrm{~F}$ NMR spectrum of 3-DME $\left(\mathrm{C}_{6} \mathrm{D}_{6}, 282 \mathrm{MHz}\right)-$ Method 1 


\section{Synthesis of $\left[\mathrm{CF}_{3}-\mathrm{ONO}\right] \mathrm{Nb}\left(\mathrm{O}^{t} \mathrm{Bu}\right)_{2}(4)$}

To $0.213 \mathrm{~g}$ of 3-OEt $\mathbf{2}(0.279 \mathrm{mmol})$ dissolved in $3 \mathrm{~mL}$ pentane chilled to $-35^{\circ} \mathrm{C}$, solid $\mathrm{LiO}{ }^{t} \mathrm{Bu}$ ( $0.052 \mathrm{~g}, 2.1$ equiv) was added in portions with constant stirring. The reaction mixture changes color from maroon to yellow-brown over $10 \mathrm{~min}$. The reaction was stirred for $4 \mathrm{~h}$ and then filtered through a pad of Celite. All volatiles were removed in vacuo to give $\mathbf{4}$ as a yellow powder. The product was recrystallized from pentane. (Yield: $0.120 \mathrm{~g}, 56.2 \%$ )

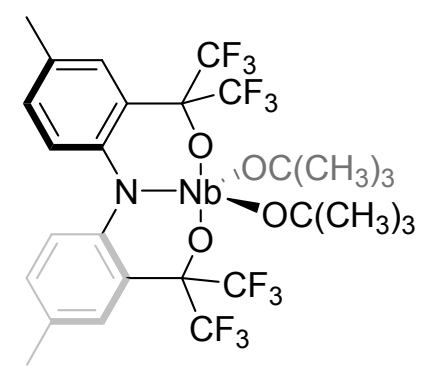

\section{4}

${ }^{1} \mathrm{H}$ NMR $\left(500 \mathrm{MHz}, \mathrm{C}_{6} \mathrm{D}_{6}\right) \delta 7.63(\mathrm{~s}, 2 \mathrm{H}, \mathrm{Ar}-H), 6.77\left(\mathrm{~d}, 2 \mathrm{H},{ }^{3} J_{\mathrm{HH}}=8.5 \mathrm{~Hz}, \mathrm{Ar}-H\right), 6.72(\mathrm{dd}, 2 \mathrm{H}$, $\left.{ }^{3} J_{\mathrm{HH}}=8.5,{ }^{4} J_{\mathrm{HH}}=1.9 \mathrm{~Hz}, \mathrm{Ar}-\mathrm{H}\right), 1.96\left(\mathrm{~s}, 6 \mathrm{H}, \mathrm{Ar}-\mathrm{C} H_{3}\right), 1.18\left(\mathrm{~s}, 18 \mathrm{H},-\mathrm{OC}\left(\mathrm{CH}_{3}\right)_{3}\right) \mathrm{ppm}$.

${ }^{13} \mathrm{C}$ determined by ${ }^{1} \mathrm{H}-{ }^{13} \mathrm{C}$ gHMBC experiment: (500 MHz, $\left.\mathrm{C}_{6} \mathrm{D}_{6}\right) \delta 148.6(\mathrm{Ar}-\mathrm{C}), 131.7$ (Ar-C), $131.0(\mathrm{Ar}-\mathrm{C}), 126.8(\mathrm{Ar}-\mathrm{C}), 125.4(\mathrm{Ar}-\mathrm{C}), 124.7(\mathrm{Ar}-\mathrm{C}), 88.3\left(-\mathrm{OC}\left(\mathrm{CH}_{3}\right)_{3}\right), 83.1\left(-\mathrm{OC}\left(\mathrm{CF}_{3}\right)_{2}\right)$, $30.0\left(-\mathrm{OC}\left(\mathrm{CH}_{3}\right)_{3}\right), 20.2\left(\mathrm{Ar}-\mathrm{CH}_{3}\right) \mathrm{ppm}$.

${ }^{19} \mathrm{~F} \mathrm{NMR}\left(282 \mathrm{MHz}, \mathrm{C}_{6} \mathrm{D}_{6}\right) \delta-71.2\left(\mathrm{q}, 6 \mathrm{~F},{ }^{4} J_{\mathrm{FF}}=9.8 \mathrm{~Hz}\right),-75.5\left(\mathrm{q}, 6 \mathrm{~F},{ }^{4} J_{\mathrm{FF}}=9.7 \mathrm{~Hz}\right) \mathrm{ppm}$.

Anal. Calcd. for $\mathrm{C}_{28} \mathrm{H}_{30} \mathrm{~F}_{12} \mathrm{NNbO}_{4}(765.44 \mathrm{~g} / \mathrm{mol}): \mathrm{C}, 43.94 \%$; H, 3.95\%; N, 1.83. Found: $\mathrm{C}$, $44.79 \% ; \mathrm{H}, 4.21 \%$; N, 2.05\%. 
NMR Characterization of $\left[\mathrm{CF}_{3}-\mathrm{ONO}\right] \mathrm{Nb}\left(\mathrm{O}^{t} \mathrm{Bu}\right)_{2}(4)$

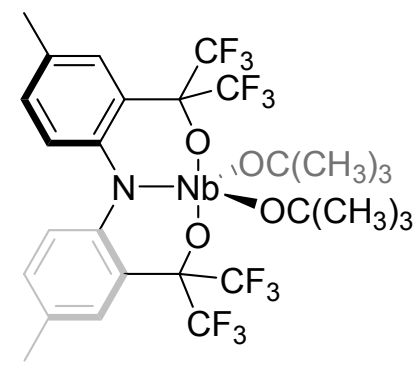

4

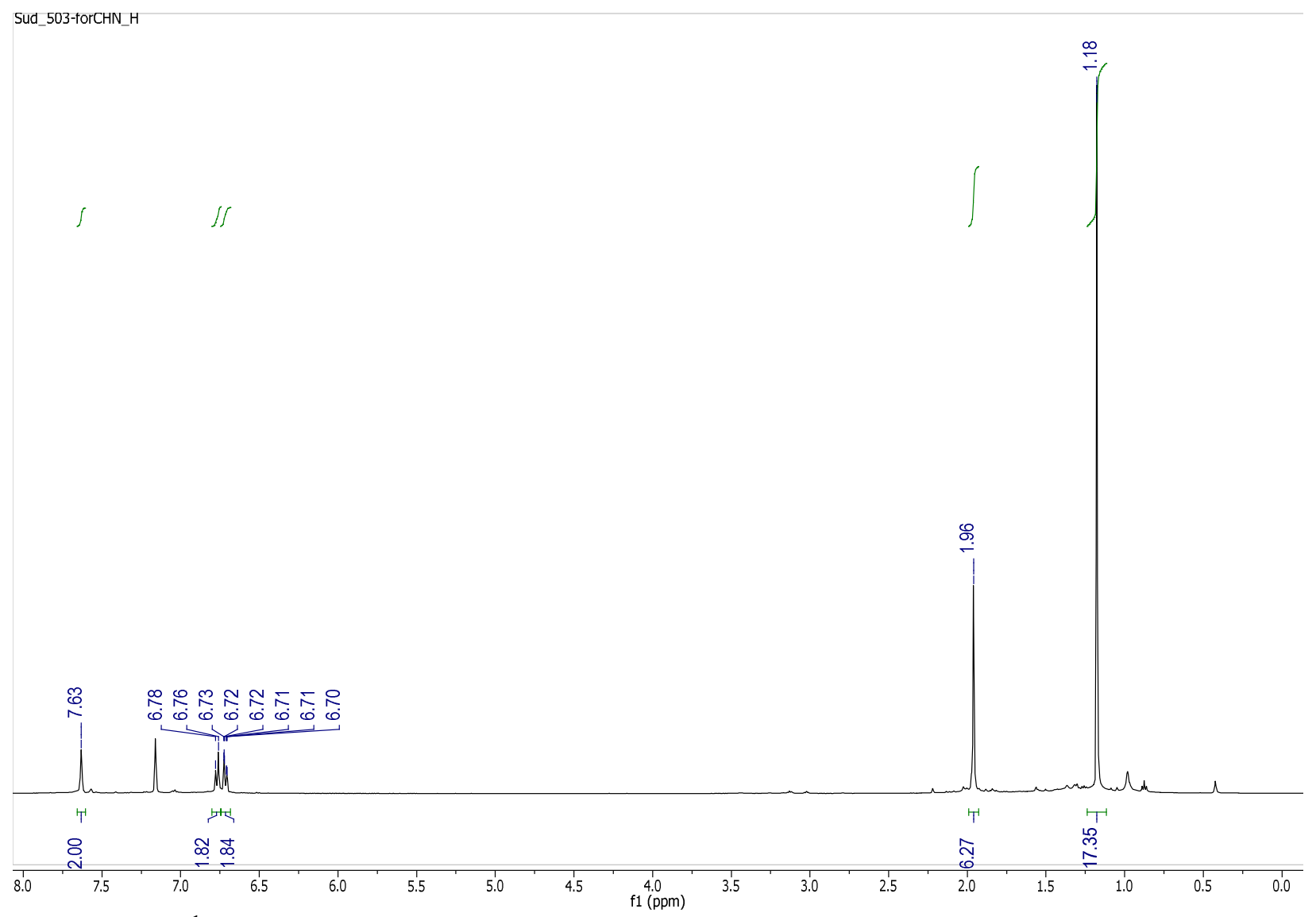

Figure S38. ${ }^{1} \mathrm{H}$ NMR spectrum of $4\left(\mathrm{C}_{6} \mathrm{D}_{6}, 500 \mathrm{MHz}\right)$ 


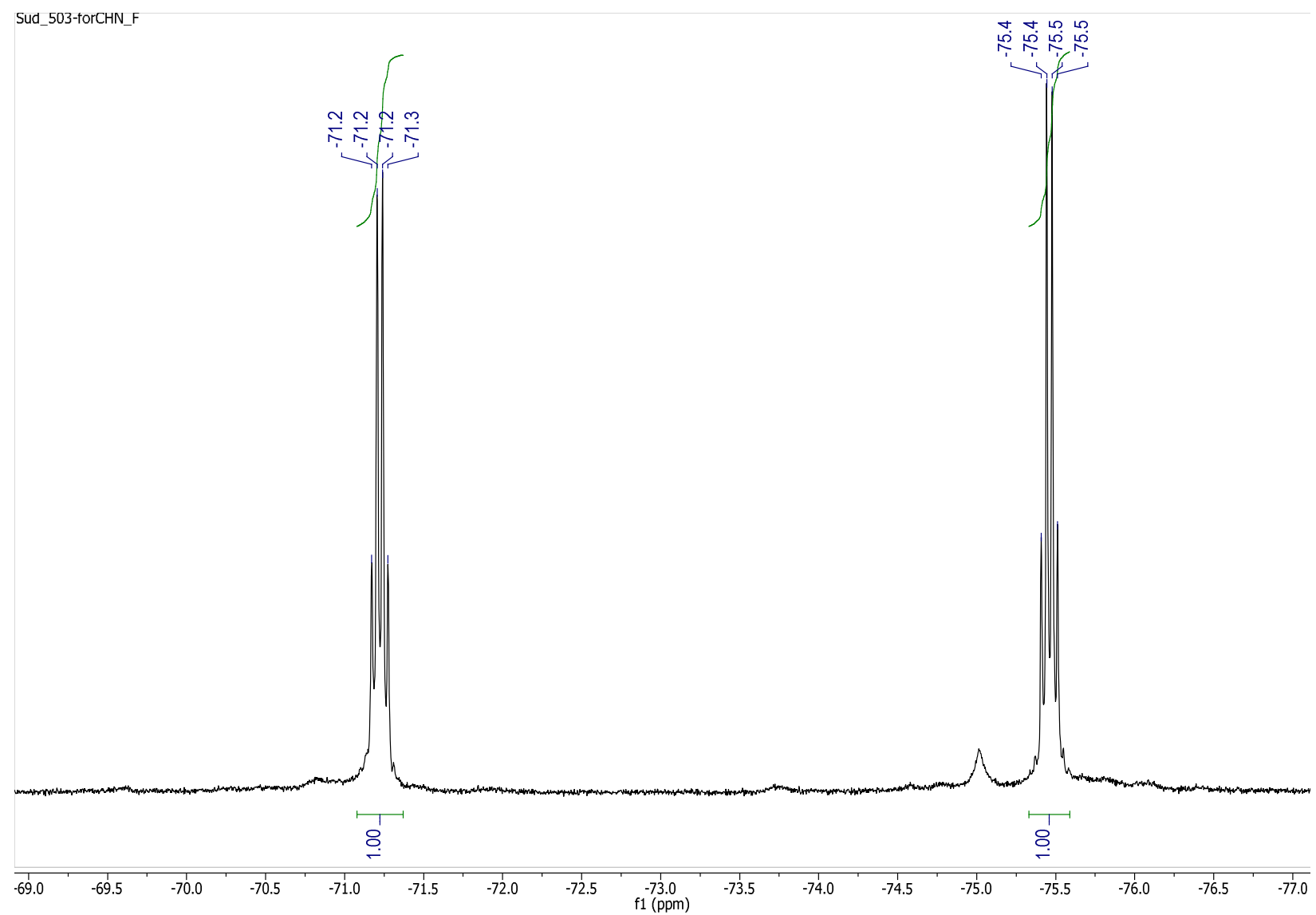

Figure S39. ${ }^{19} \mathrm{~F}$ NMR spectrum of $4\left(\mathrm{C}_{6} \mathrm{D}_{6}, 282 \mathrm{MHz}\right)$ 


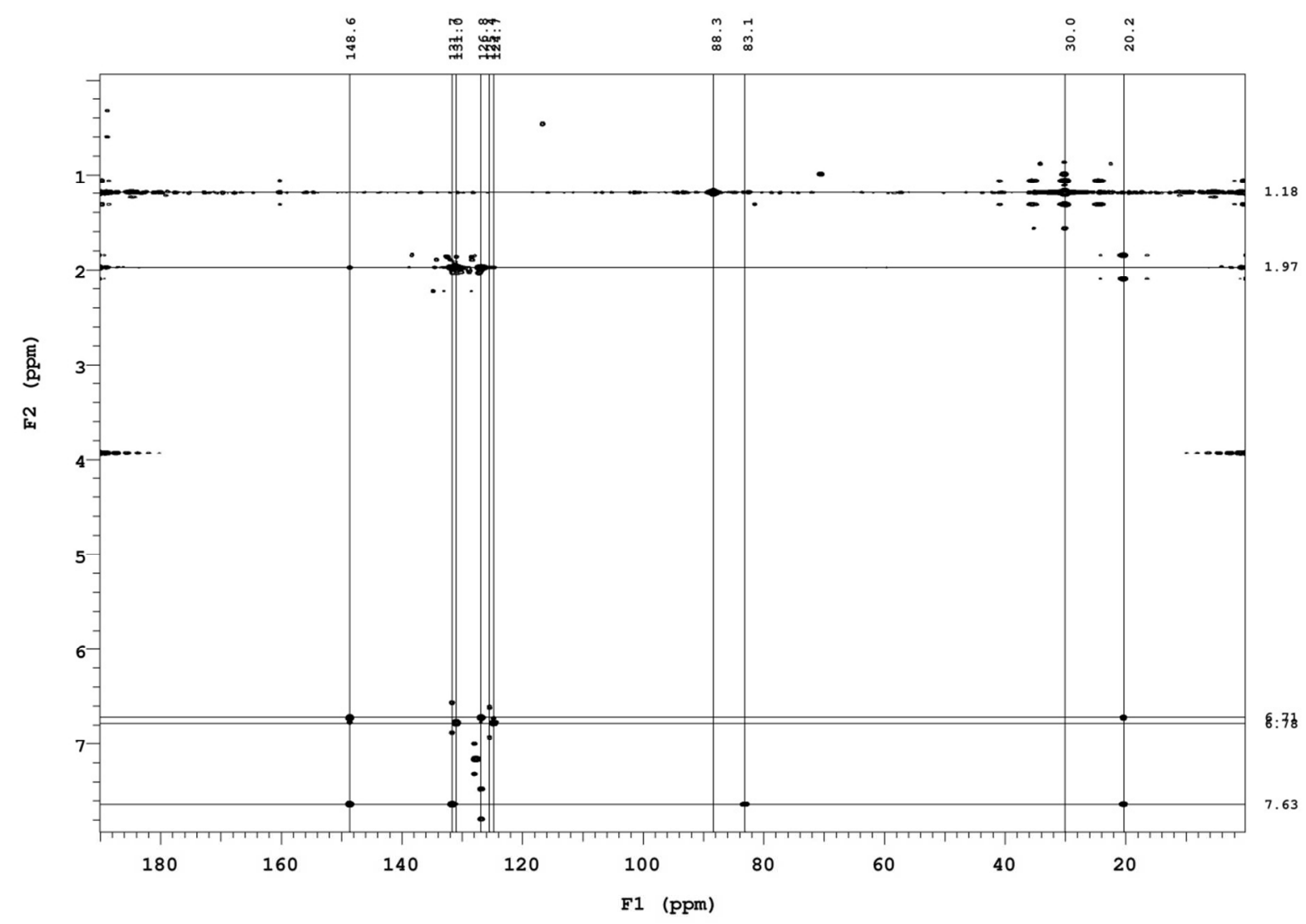

Figure S40. ${ }^{1} \mathrm{H}_{-}{ }^{13} \mathrm{C} g \mathrm{HMBC}$ of $4\left(\mathrm{C}_{6} \mathrm{D}_{6}, 500 \mathrm{MHz}\right)$ 


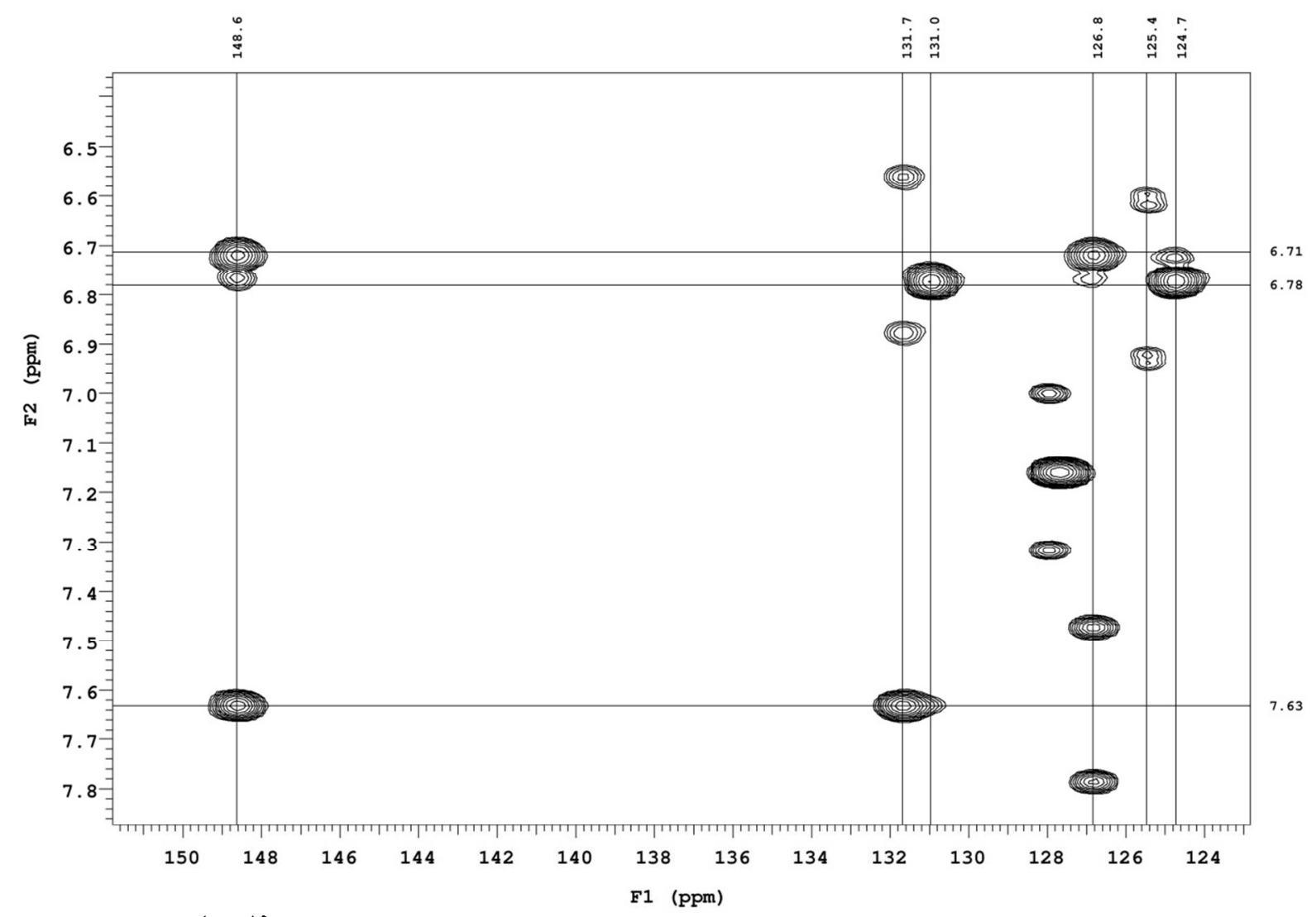

Figure S41. ${ }^{1} \mathrm{H}_{-}{ }^{13} \mathrm{C} g \mathrm{HMBC}$ of $4-$ Expansion $\left(\mathrm{C}_{6} \mathrm{D}_{6}, 500 \mathrm{MHz}\right)$ 


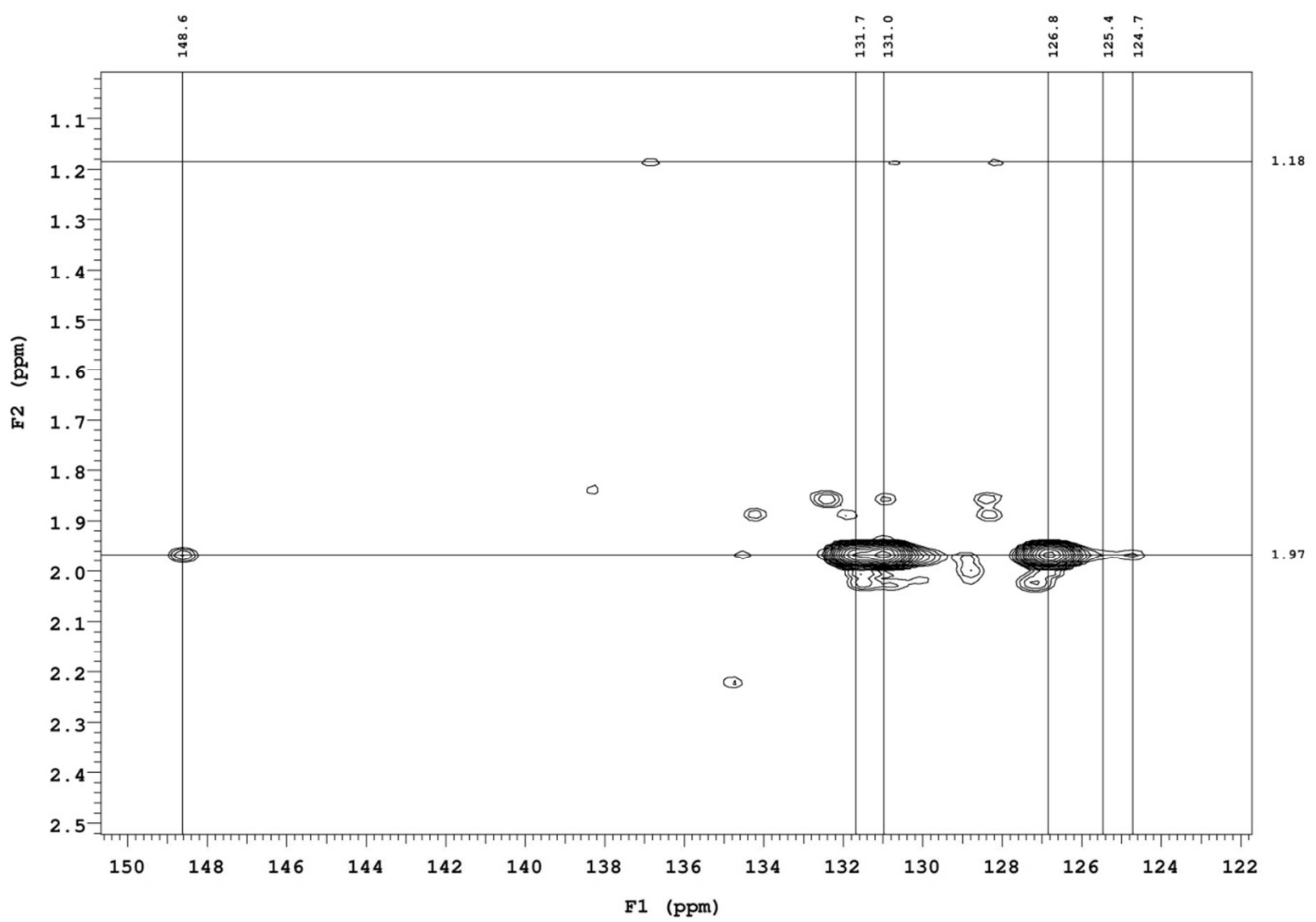

Figure S42. ${ }^{1} \mathrm{H}_{-}{ }^{13} \mathrm{C} g \mathrm{HMBC}$ of $4-$ Expansion $\left(\mathrm{C}_{6} \mathrm{D}_{6}, 500 \mathrm{MHz}\right)$ 


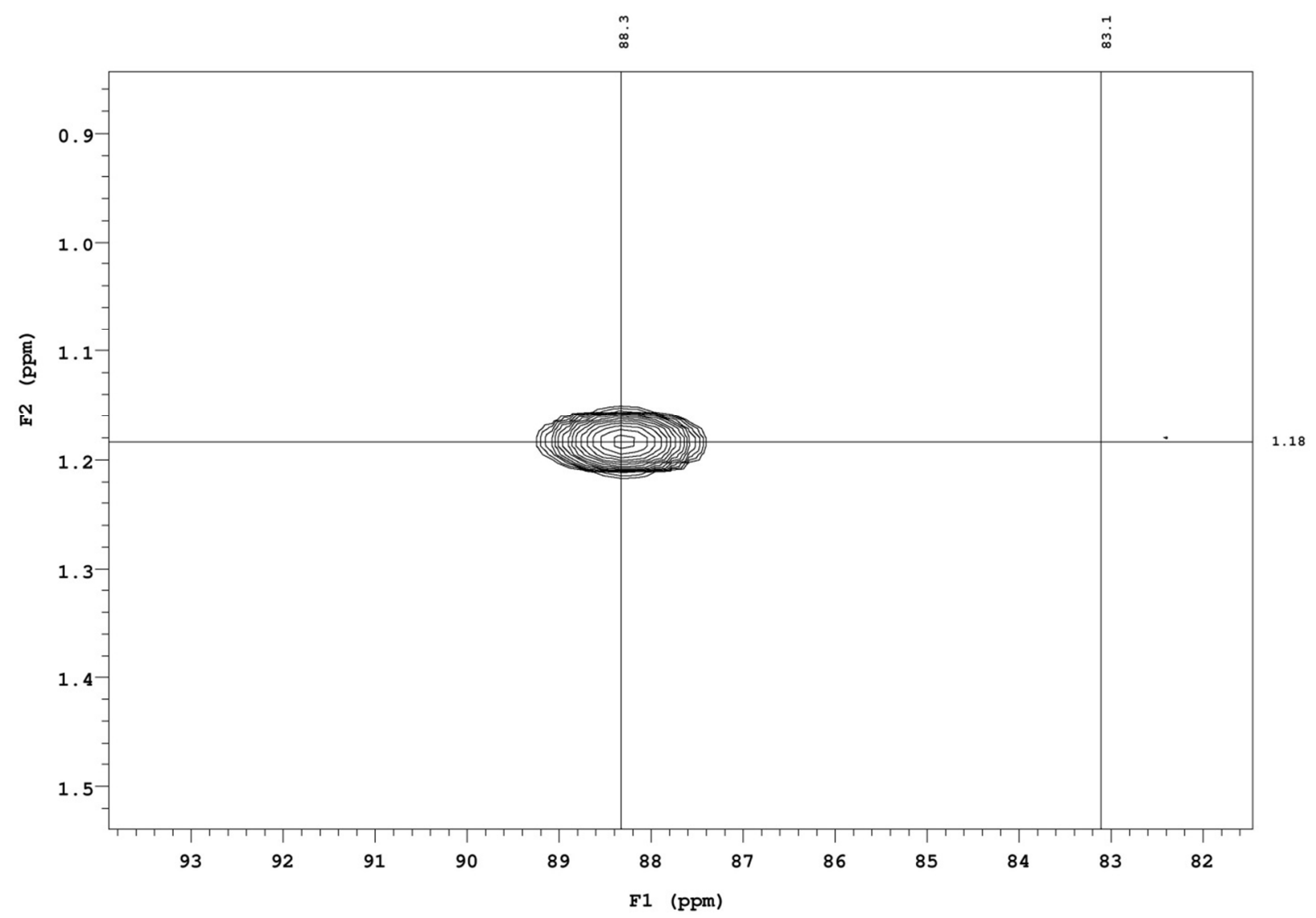

Figure S43. ${ }^{1} \mathrm{H}_{-}{ }^{13} \mathrm{C} g \mathrm{HMBC}$ of $4-$ Expansion $\left(\mathrm{C}_{6} \mathrm{D}_{6}, 500 \mathrm{MHz}\right)$ 


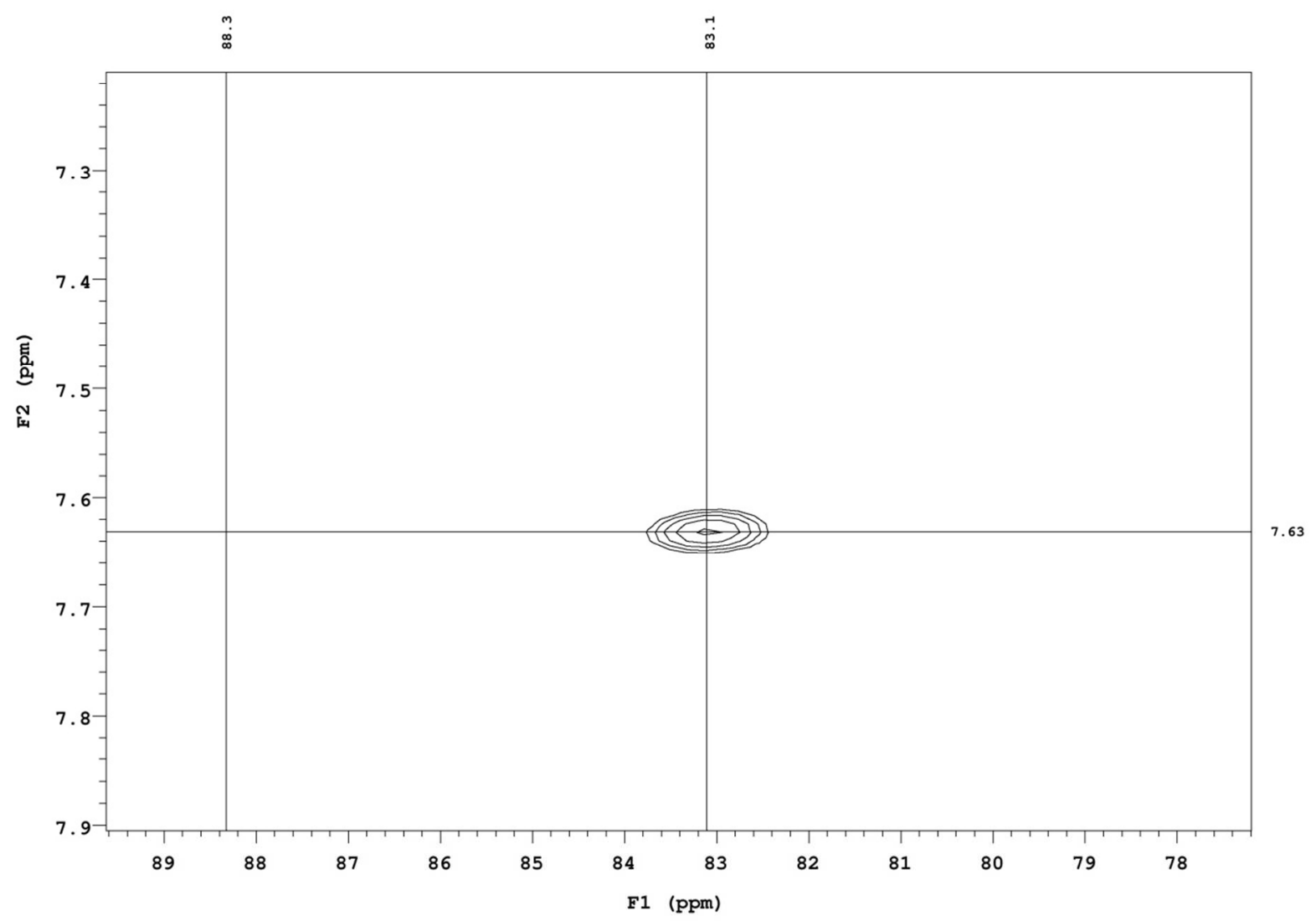

Figure S44. ${ }^{1} \mathrm{H}-{ }^{13} \mathrm{C} g \mathrm{HMBC}$ of 4 - Expansion $\left(\mathrm{C}_{6} \mathrm{D}_{6}, 500 \mathrm{MHz}\right)$ 


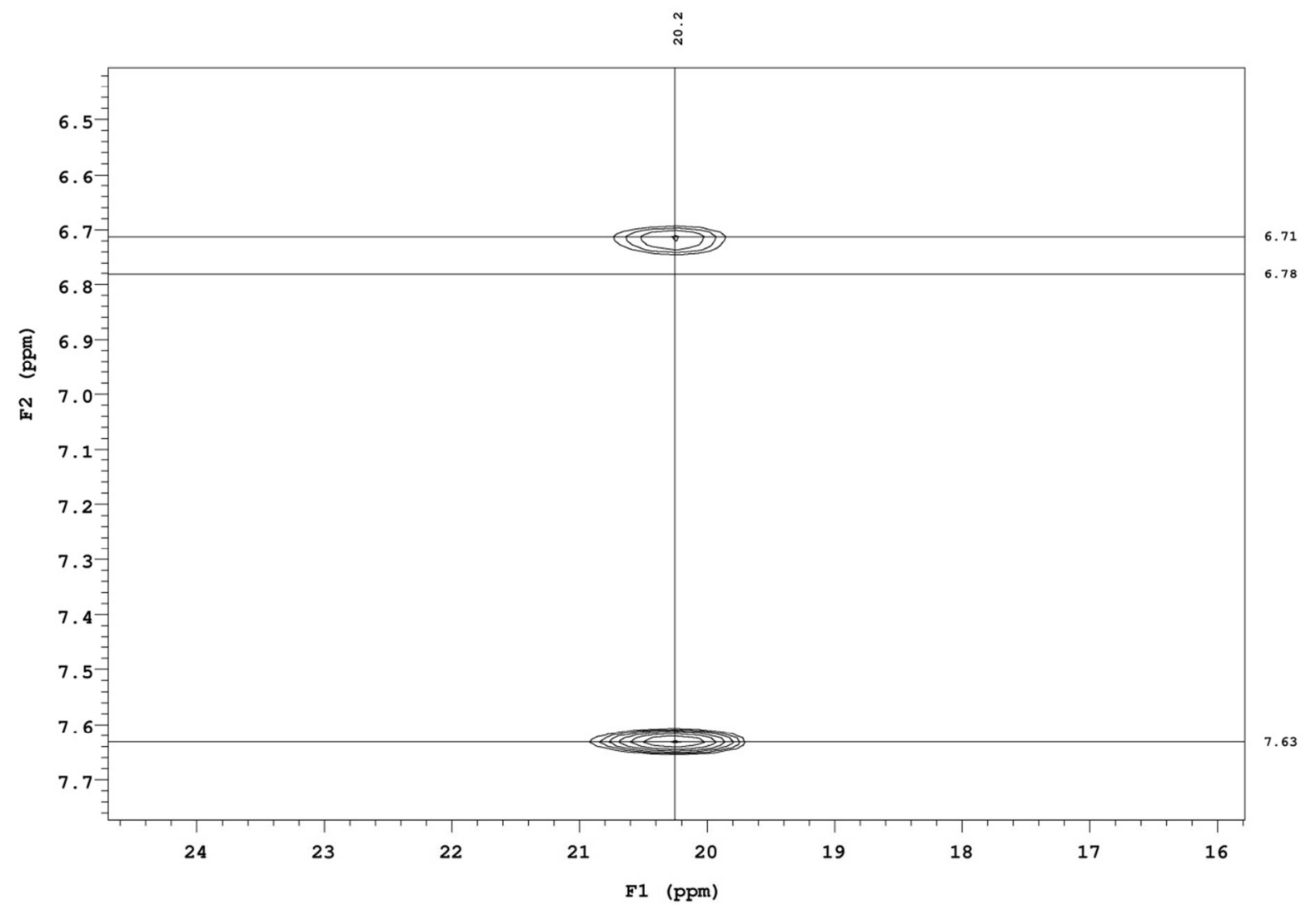

Figure S45. ${ }^{1} \mathrm{H}-{ }^{13} \mathrm{C} g \mathrm{HMBC}$ of $4-$ Expansion $\left(\mathrm{C}_{6} \mathrm{D}_{6}, 500 \mathrm{MHz}\right)$ 


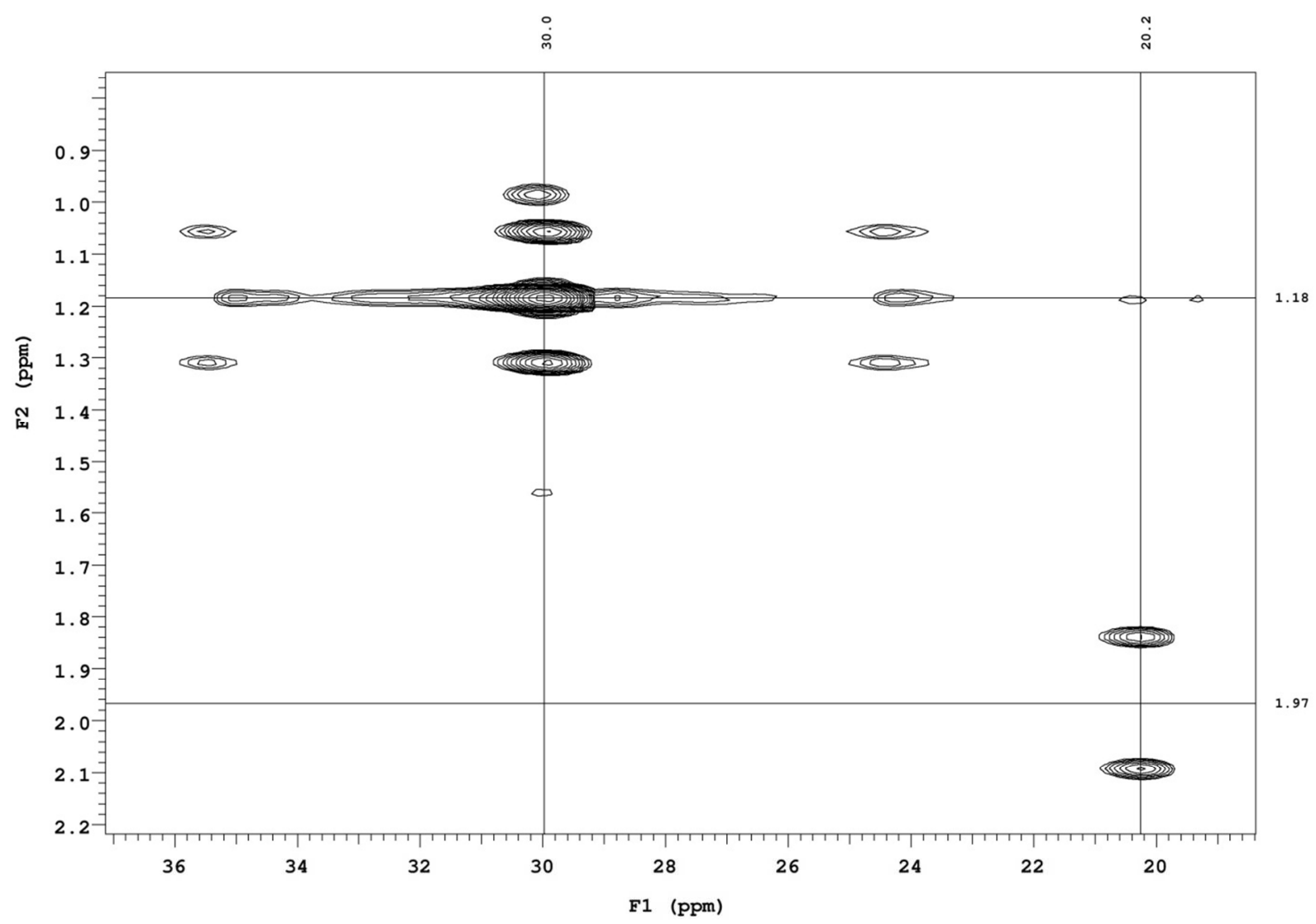

Figure S46. ${ }^{1} \mathrm{H}-{ }^{13} \mathrm{C} g \mathrm{HMBC}$ of $4-$ Expansion $\left(\mathrm{C}_{6} \mathrm{D}_{6}, 500 \mathrm{MHz}\right)$ 


\section{Synthesis of $\left[\mathrm{CF}_{3}-\mathrm{ONO}\right] \mathrm{Nb}\left(\mathrm{CH}_{2} \mathrm{C}_{6} \mathrm{H}_{5}\right)_{2}(5)$}

To $0.084 \mathrm{~g}(0.101 \mathrm{mmol})$ of 3-OEt $\mathbf{2}_{2}$ in $2 \mathrm{~mL} \mathrm{Et}_{2} \mathrm{O}$ chilled to $-35^{\circ} \mathrm{C}, 220 \mu \mathrm{L}$ benzylmagnesium chloride solution ( $0.220 \mathrm{mmol}, 2$ equiv) was added in drops. An instant color change from maroon to red was observed. The reaction mixture was stirred for $4 \mathrm{~h}$ after which all volatiles were removed in vacuo, and the tacky residue was repeatedly extracted with pentane until colorless. The extracts were filtered through a pad of Celite, and all volatiles evaporated to give 5 as a deep red powder. (Yield: $0.065 \mathrm{~g}, 80.3 \%$ )

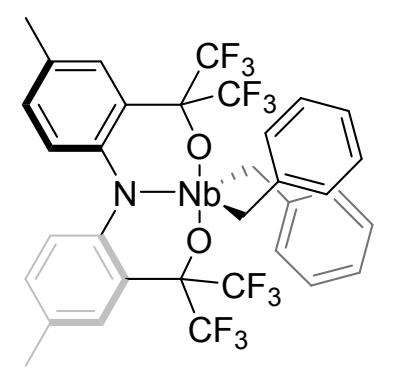

5

${ }^{1} \mathrm{H}$ NMR (500 MHz, $\left.\mathrm{C}_{6} \mathrm{D}_{6}\right) \delta 7.44$ (s, 2H, Ar- $H$ ), $6.96-6.83$ (m, 10H, Ar- $H$ - benzyl fragment), $6.66\left(\mathrm{dd}, 2 \mathrm{H},{ }^{3} J_{\mathrm{HH}}=8.5,{ }^{4} J_{\mathrm{HH}}=2.1 \mathrm{~Hz}\right), 6.61\left(\mathrm{~d}, 2 \mathrm{H},{ }^{3} J_{\mathrm{HH}}=8.5 \mathrm{~Hz}, \mathrm{Ar}-H\right), 2.52\left(\mathrm{~s}, 4 \mathrm{H},-\mathrm{CH}_{2}-\right.$ benzyl), 1.97 (s, 6H, $\left.\mathrm{Ar}-\mathrm{CH}_{3}\right)$ ppm.

${ }^{13} \mathrm{C}\left\{{ }^{1} \mathrm{H}\right\}$ NMR (126 MHz, $\left.\mathrm{C}_{6} \mathrm{D}_{6}\right) \delta 148.8(\operatorname{Ar}-C), 132.8(\operatorname{Ar}-C), 132.4(\operatorname{Ar}-C), 131.9$ (Ar-C), $131.1(\mathrm{Ar}-C), 129.5(\mathrm{Ar}-C), 127.6(\mathrm{Ar}-C), 126.4(\mathrm{Ar}-C), 125.6(\mathrm{Ar}-C), 123.3(\mathrm{Ar}-C), 83.6$ $\left(-\mathrm{OC}\left(\mathrm{CF}_{3}\right)_{2}\right), 68.9\left(-\mathrm{CH}_{2} \mathrm{C}_{6} \mathrm{H}_{5}\right), 20.1\left(\mathrm{Ar}-\mathrm{CH}_{3}\right) \mathrm{ppm}$.

${ }^{19} \mathrm{~F} \mathrm{NMR}\left(282 \mathrm{MHz}, \mathrm{C}_{6} \mathrm{D}_{6}\right) \delta-70.4\left(\mathrm{q}, 6 \mathrm{~F}, 4 J_{\mathrm{FF}}=9.9 \mathrm{~Hz}\right),-74.9\left(\mathrm{q}, 6 \mathrm{~F}, 4 J_{\mathrm{FF}}=9.9 \mathrm{~Hz}\right) \mathrm{ppm}$. Anal. Calcd. for $\mathrm{C}_{34} \mathrm{H}_{26} \mathrm{~F}_{12} \mathrm{NNbO}_{2}(801.47 \mathrm{~g} / \mathrm{mol})$ : C, $50.95 \% ; \mathrm{H}, 3.27 \%$; N, 1.75\%. Found: $\mathrm{C}$, $50.75 \%, \mathrm{H}, 3.10 \%, \mathrm{~N}, 1.85 \%$ 
NMR Characterization of $\left[\mathrm{CF}_{3}-\mathrm{ONO}\right] \mathrm{Nb}\left(\mathrm{CH}_{2} \mathrm{C}_{6} \mathrm{H}_{5}\right)_{2},(5)$
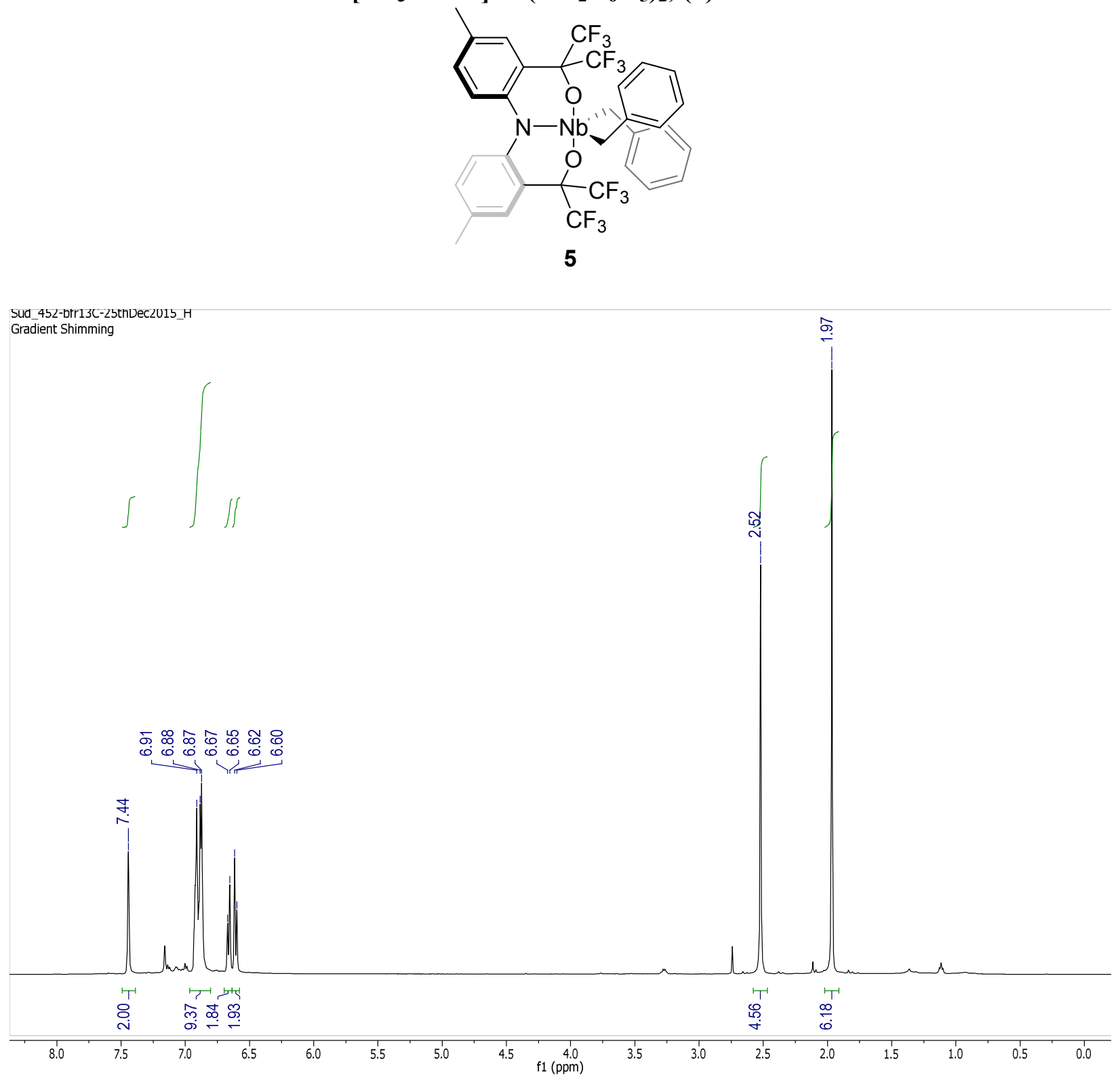

Figure S47. ${ }^{1} \mathrm{H}$ NMR spectrum of $5\left(\mathrm{C}_{6} \mathrm{D}_{6}, 500 \mathrm{MHz}\right)$ 


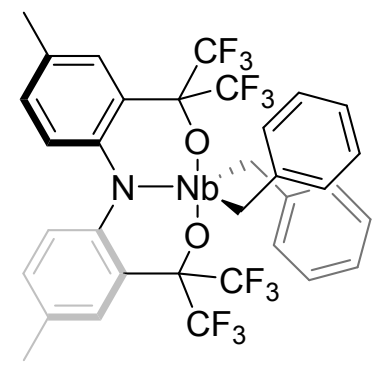

sua_4b_-10000scans-26tnueczu1b_13C

5

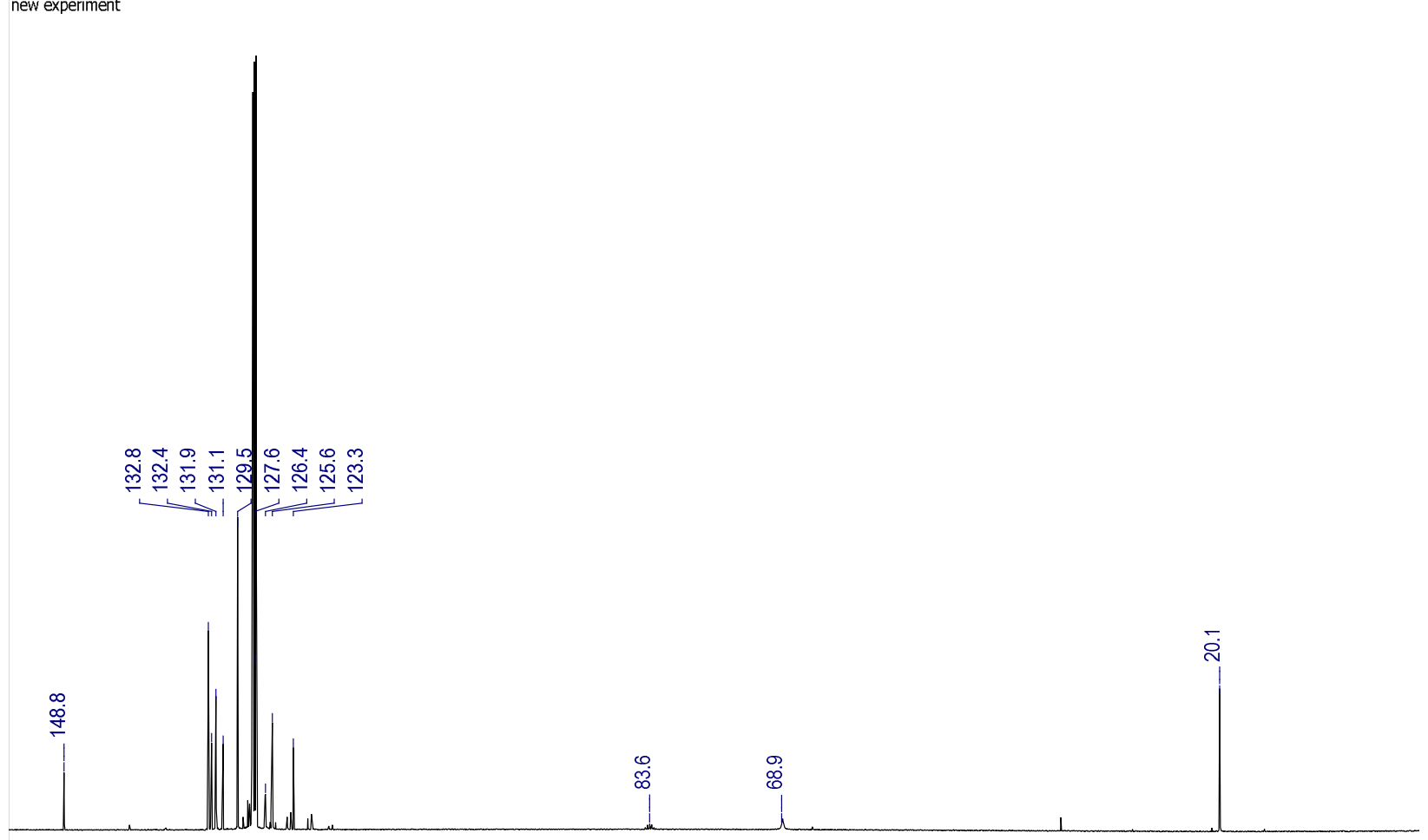

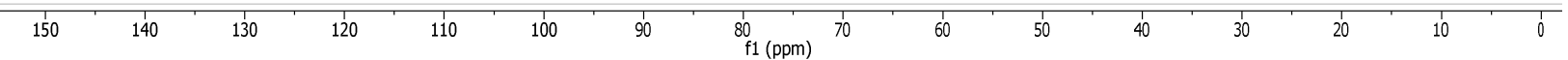

Figure S48. ${ }^{13} \mathrm{C}\left\{{ }^{1} \mathrm{H}\right\} \mathrm{NMR}$ spectrum of $5\left(\mathrm{C}_{6} \mathrm{D}_{6}, 126 \mathrm{MHz}\right)$ 


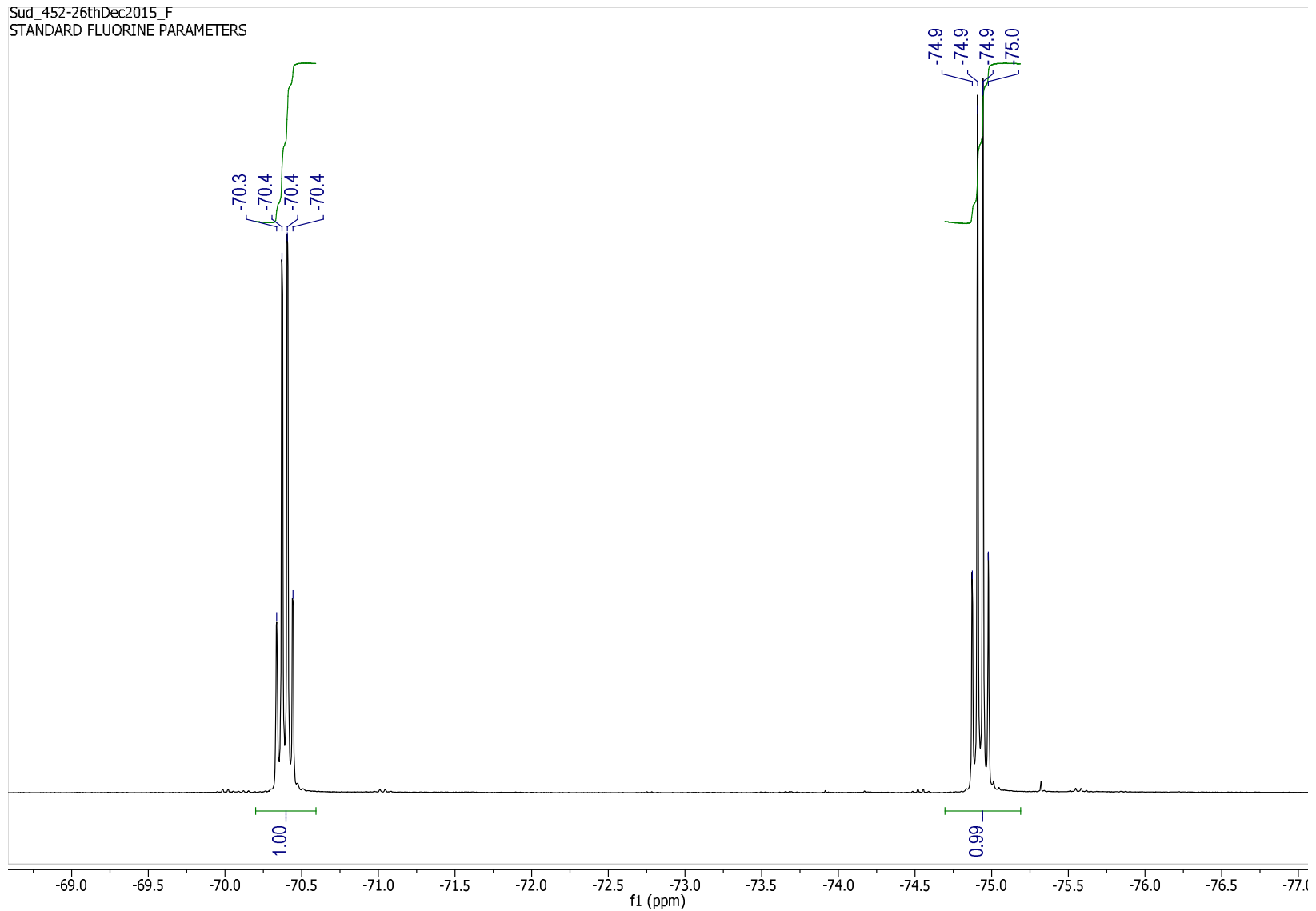

Figure S49. ${ }^{19} \mathrm{~F}$ NMR spectrum of $5\left(\mathrm{C}_{6} \mathrm{D}_{6}, 282 \mathrm{MHz}\right)$ 


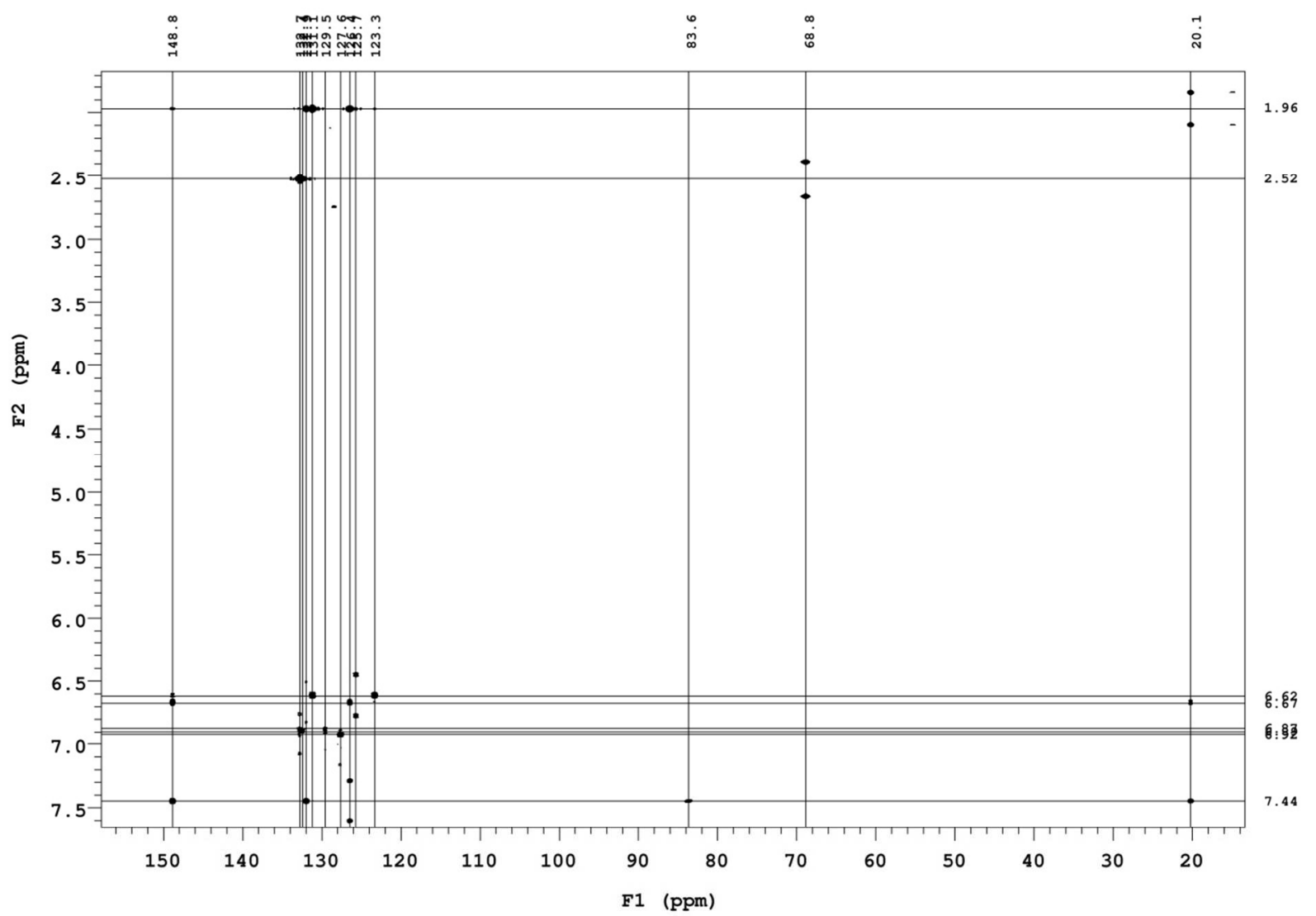

Figure S50. ${ }^{1} \mathrm{H}-{ }^{13} \mathrm{C} g \mathrm{HMBC}$ spectrum of $5\left(\mathrm{C}_{6} \mathrm{D}_{6}, 500 \mathrm{MHz}\right)$ 


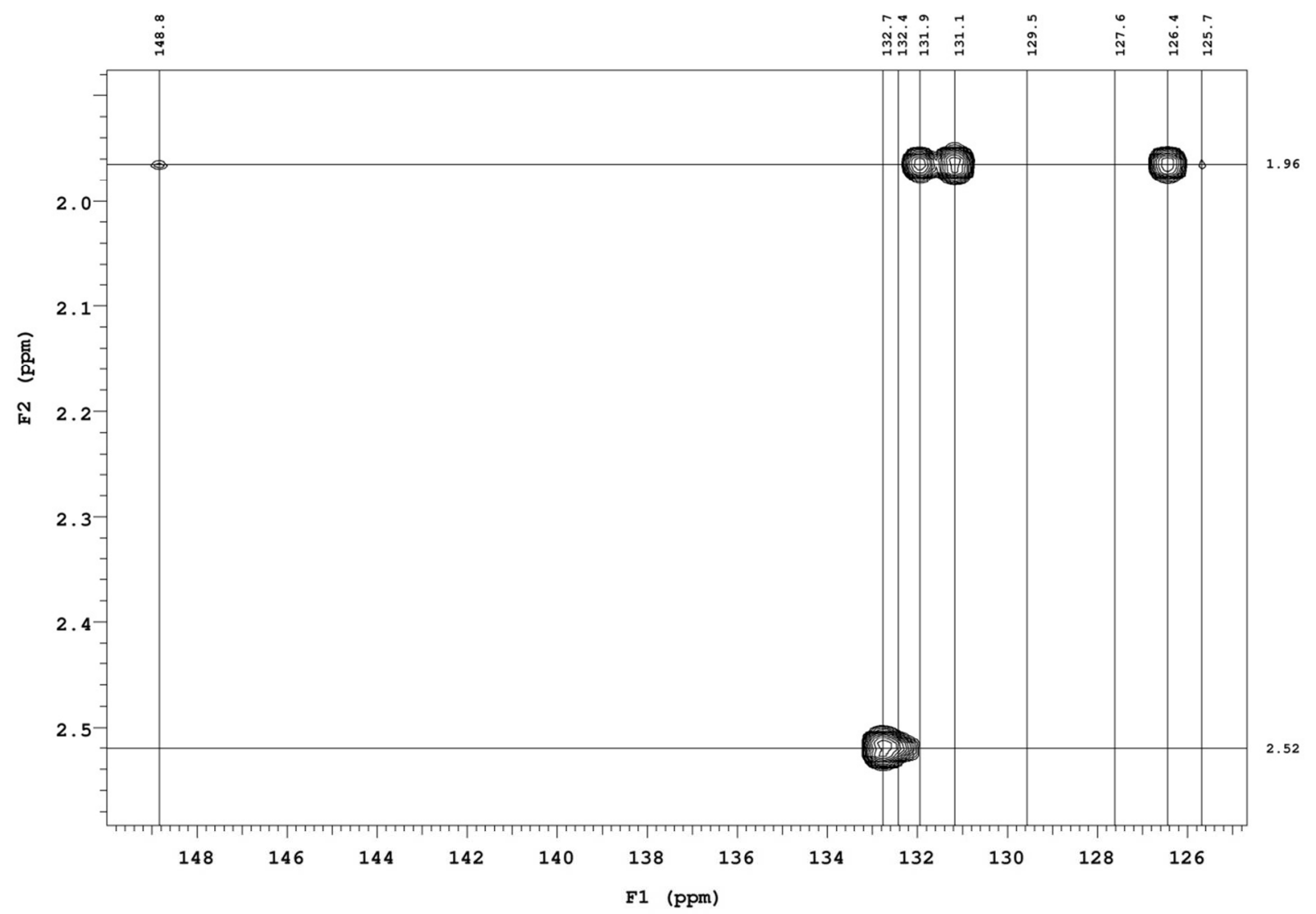

Figure S51. ${ }^{1} \mathrm{H}-{ }^{13} \mathrm{C} g \mathrm{HMBC}$ spectrum of $5-$ Expansion $\left(\mathrm{C}_{6} \mathrm{D}_{6}, 500 \mathrm{MHz}\right)$ 


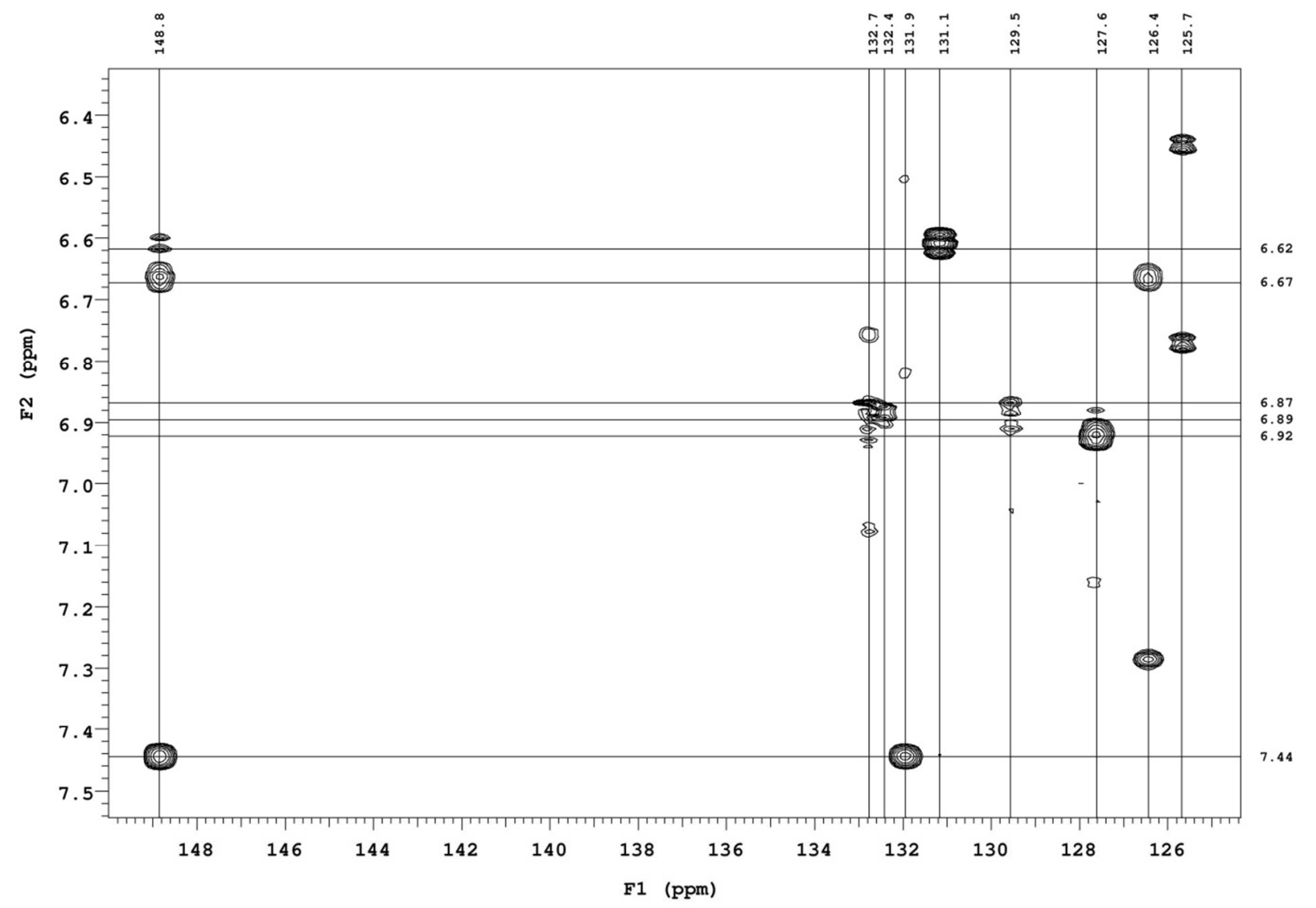

Figure S52. ${ }^{1} \mathrm{H}-{ }^{13} \mathrm{C} g \mathrm{HMBC}$ spectrum of 5 - Expansion $\left(\mathrm{C}_{6} \mathrm{D}_{6}, 500 \mathrm{MHz}\right)$ 


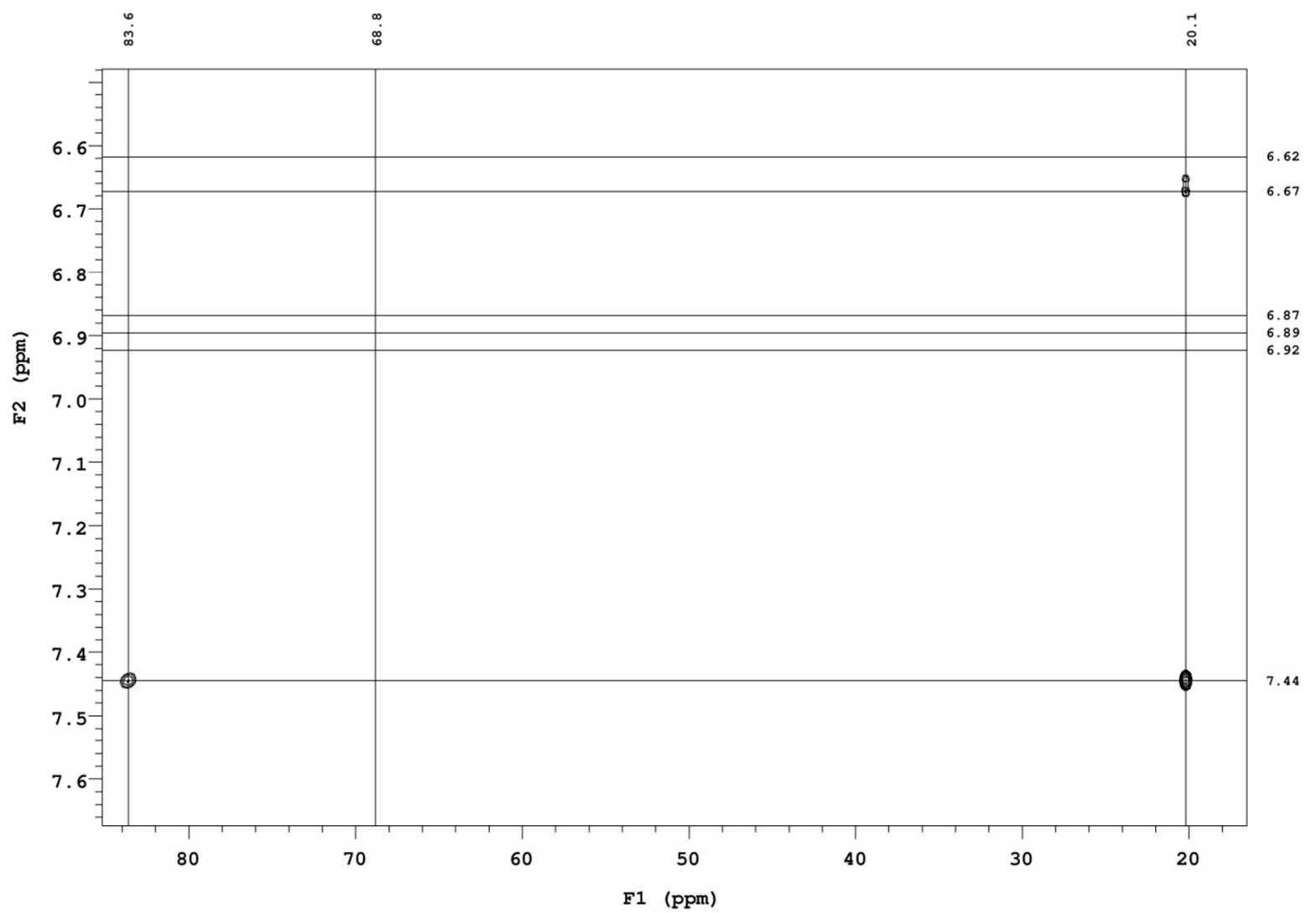

Figure S53. ${ }^{1} \mathrm{H}-{ }^{13} \mathrm{C} g \mathrm{HMBC}$ spectrum of $5-$ Expansion $\left(\mathrm{C}_{6} \mathrm{D}_{6}, 500 \mathrm{MHz}\right)$ 


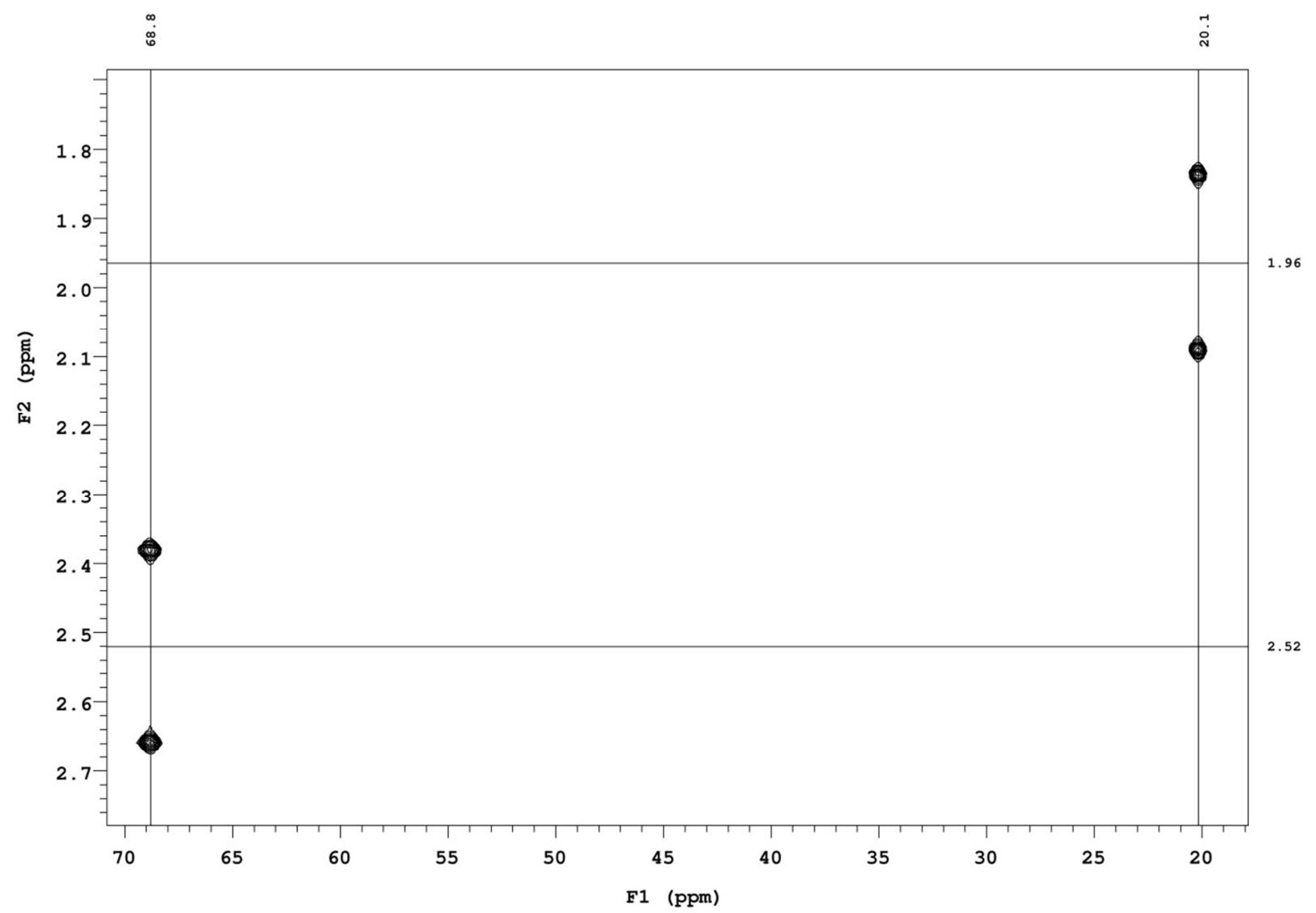

Figure S54. ${ }^{1} \mathrm{H}-{ }^{13} \mathrm{C} g \mathrm{HMBC}$ spectrum of 5 - Expansion $\left(\mathrm{C}_{6} \mathrm{D}_{6}, 500 \mathrm{MHz}\right)$ 

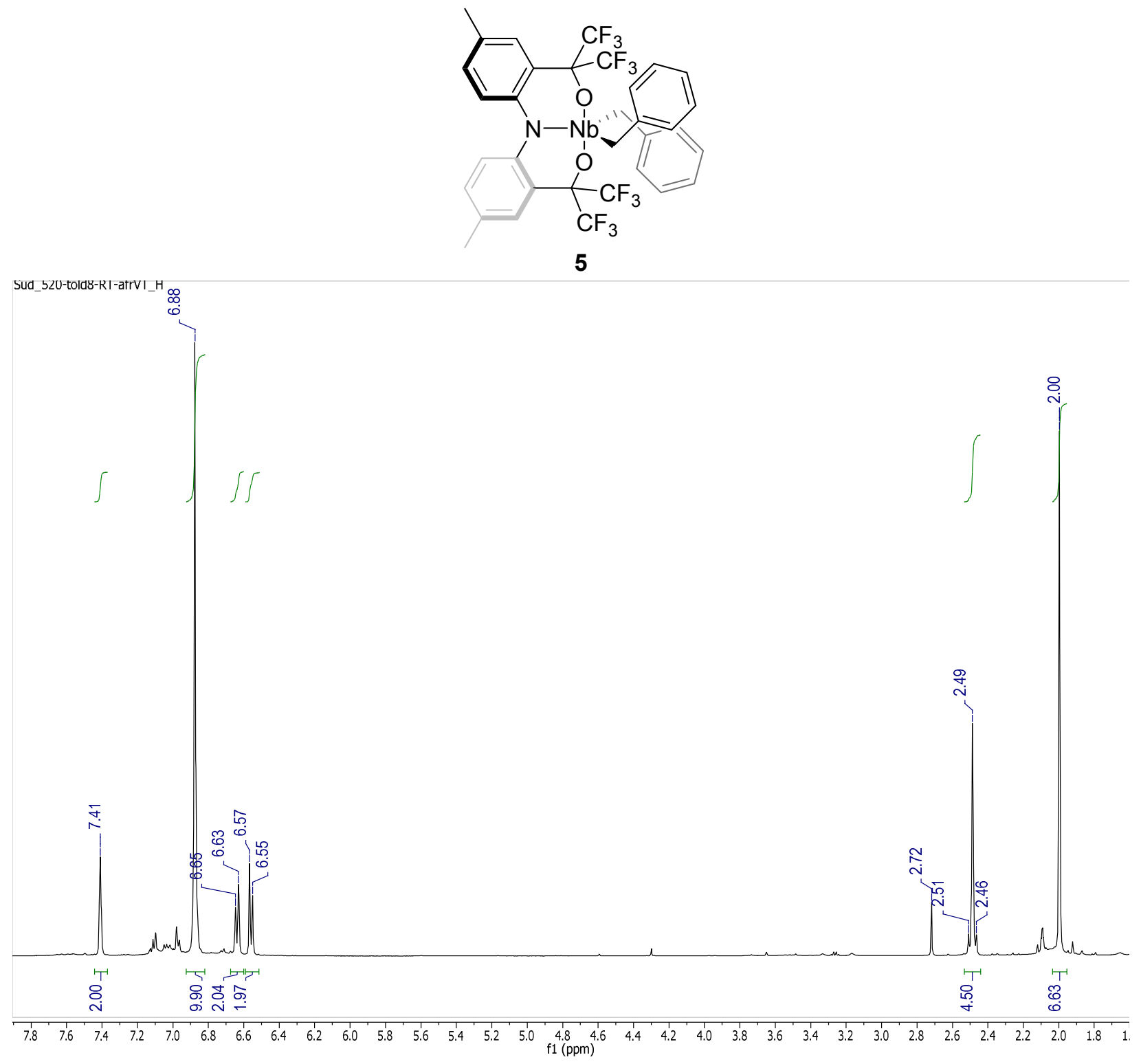

Figure S55. ${ }^{1} \mathrm{H}$ NMR spectrum of 5 (toluene- $d_{8}, 500 \mathrm{MHz}$ ) 


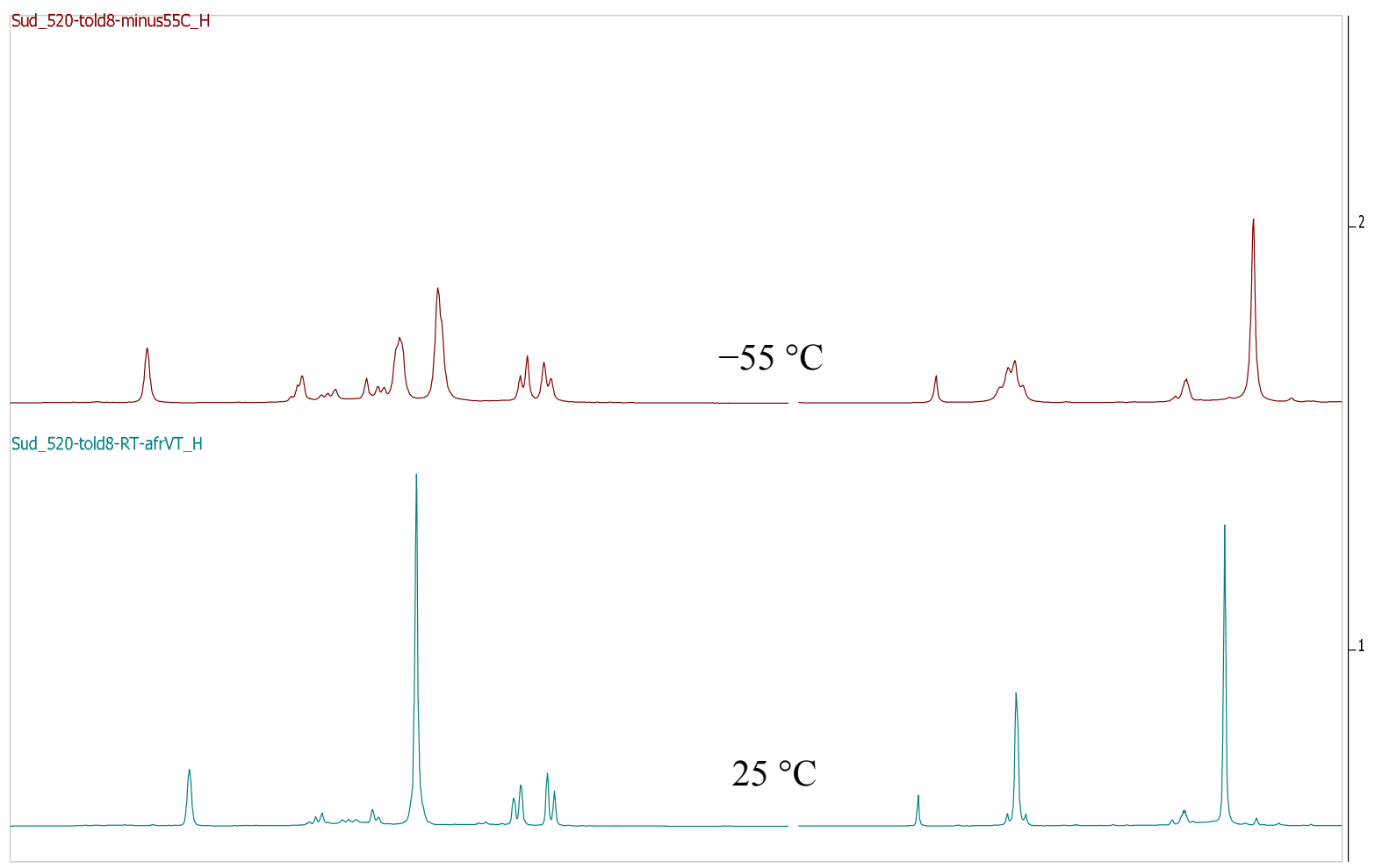

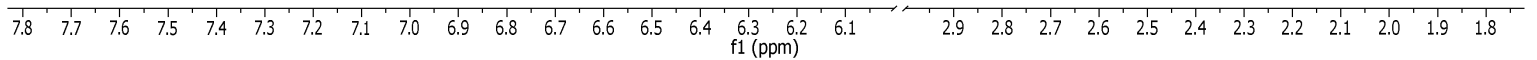

Figure S56. Variable temperature ${ }^{1} \mathrm{H}$ NMR spectra of 5 (toluene- $d_{8}, 500 \mathrm{MHz}$ ) - abridged (only $-55^{\circ} \mathrm{C}$ and $+25^{\circ} \mathrm{C}$ shown) 


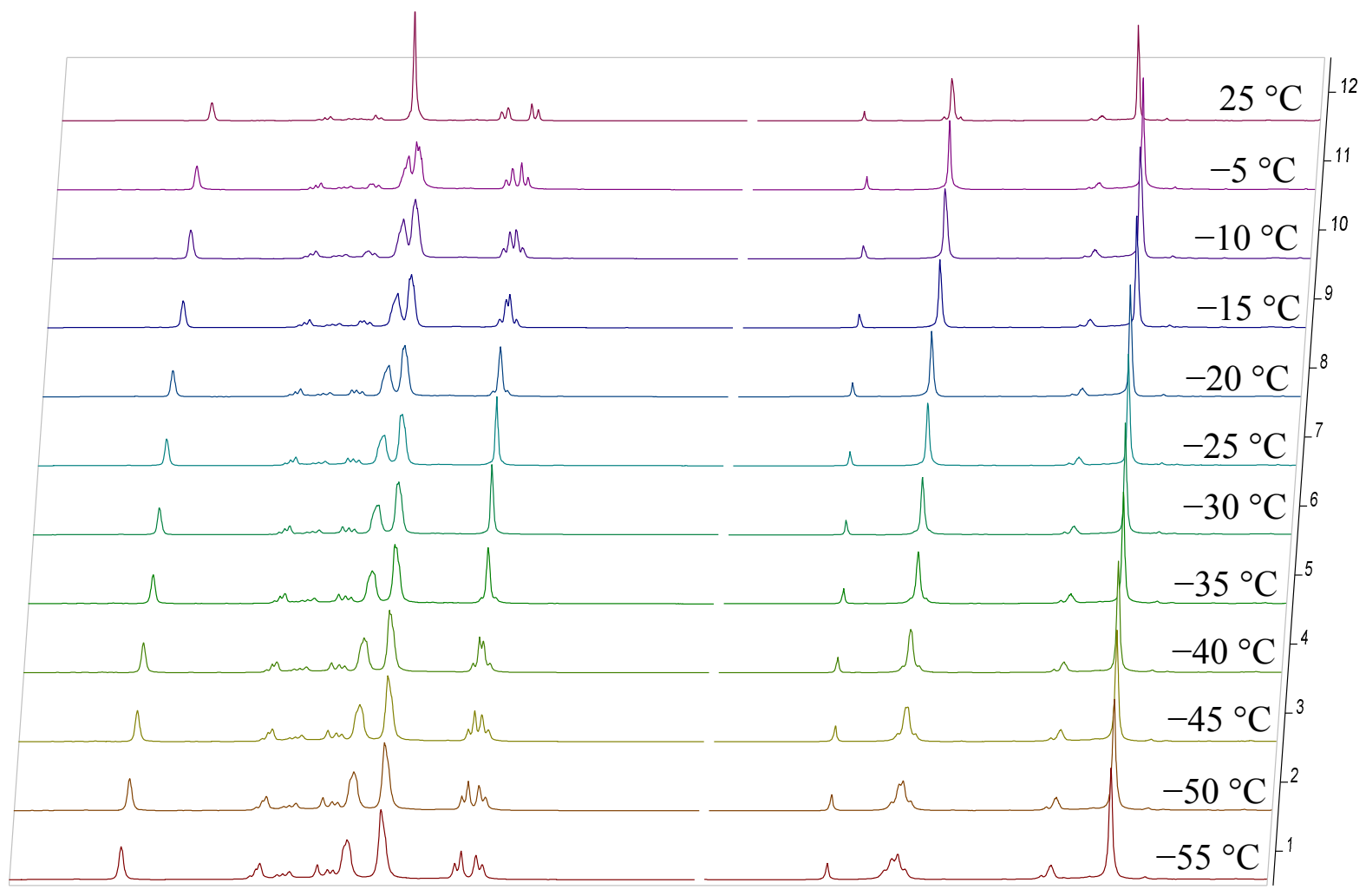

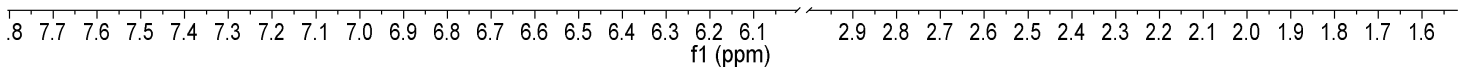

Figure S57. Variable temperature ${ }^{1} \mathrm{H}$ NMR spectra of 5 (toluene- $d_{8}, 500 \mathrm{MHz}$ ) - Full

Temperature range: $-55^{\circ} \mathrm{C}$ to $-5{ }^{\circ} \mathrm{C}, 25^{\circ} \mathrm{C}$ 


\section{Synthesis of $\left[\mathrm{CF}_{3}-\mathrm{ONO}\right] \mathrm{Nb}\left(\mathrm{CH}_{2} \mathrm{Si}\left(\mathrm{CH}_{3}\right)_{3}\right)_{2}(6)$}

In a vial, 3-OEt $\mathbf{2}_{2}(0.120 \mathrm{~g}, 0.157 \mathrm{mmol})$ was dissolved in $2 \mathrm{~mL}$ pentane and chilled to $-35{ }^{\circ} \mathrm{C}$. $\mathrm{TMSCH}_{2} \mathrm{MgCl}$ (0.314 mmol, 2 equiv.) was added in drops to the chilled solution of 3a. An immediate color change from maroon to orange was observed, with the formation of copious amounts of a precipitate. The reaction mixture was stirred for $3.5 \mathrm{~h}$ and then filtered. All volatiles were removed in vacuo to afford deep orange oil. Addition of a small amount of acetonitrile followed by evaporation to dryness gives 6 as an orange solid. (Yield: $0.103 \mathrm{~g}$, $82.6 \%)$

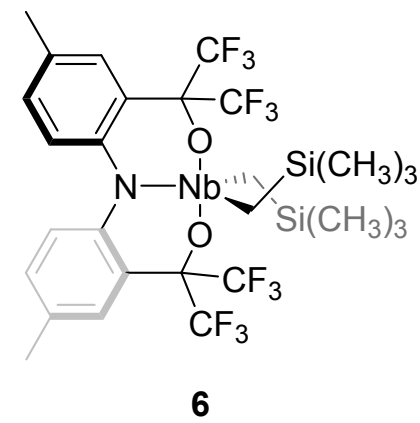

${ }^{1} \mathrm{H}$ NMR $\left(300 \mathrm{MHz}, \mathrm{C}_{6} \mathrm{D}_{6}\right) \delta 7.57(\mathrm{~s}, 2 \mathrm{H}, \mathrm{Ar}-H), 6.82\left(\mathrm{~d}, 2 \mathrm{H},{ }^{3} J_{\mathrm{HH}}=8.5 \mathrm{~Hz}, \mathrm{Ar}-H\right), 6.70(\mathrm{dd}, 2 \mathrm{H}$, $\left.{ }^{3} J_{\mathrm{HH}}=8.5,{ }^{4} J_{\mathrm{HH}}=2.0 \mathrm{~Hz}, \mathrm{Ar}-H\right), 2.21\left(\mathrm{~d}, 2 \mathrm{H},{ }^{2} J_{\mathrm{HH}}=11.3 \mathrm{~Hz},-\mathrm{CH}_{2} \mathrm{SiMe}_{3}\right), 2.14\left(\mathrm{~d}, 2 \mathrm{H},{ }^{2} J_{\mathrm{HH}}=\right.$ $\left.11.0 \mathrm{~Hz},-\mathrm{CH}_{2} \mathrm{SiMe}_{3}\right), 1.92$ (s, 6H, $\left.\mathrm{Ar}-\mathrm{CH}_{3}\right), 0.05\left(\mathrm{~s}, 18 \mathrm{H},-\mathrm{Si}\left(\mathrm{CH}_{3}\right)_{3}\right) \mathrm{ppm}$.

${ }^{13} \mathrm{C}$ determined by ${ }^{1} \mathrm{H}_{-}{ }^{13} \mathrm{C}$ gHMBC experiment: (500 MHz, $\left.\mathrm{C}_{6} \mathrm{D}_{6}\right) \delta 147.6(\mathrm{Ar}-\mathrm{C}), 132.3(\mathrm{Ar}-\mathrm{C})$, 131.5 (Ar-C), $126.9(\mathrm{Ar}-C), 126.7(\mathrm{Ar}-\mathrm{C}), 124.6(\mathrm{Ar}-\mathrm{C}), 84.1\left(-\mathrm{OC}\left(\mathrm{CF}_{3}\right)_{2}\right), 77.4\left(-\mathrm{CH}_{2} \mathrm{SiMe}_{3}\right)$, $20.2\left(\mathrm{Ar}-\mathrm{CH}_{3}\right), 0.7\left(-\mathrm{Si}\left(\mathrm{CH}_{3}\right)_{3}\right) \mathrm{ppm}$.

${ }^{19} \mathrm{~F}$ NMR $\left(282 \mathrm{MHz}, \mathrm{C}_{6} \mathrm{D}_{6}\right) \delta-70.6\left(\mathrm{q},{ }^{4} J_{\mathrm{FF}}=9.5 \mathrm{~Hz}\right),-75.1\left(\mathrm{q},{ }^{4} J_{\mathrm{FF}}=9.5 \mathrm{~Hz}\right) \mathrm{ppm}$.

Anal. Calcd. for $\mathrm{C}_{28} \mathrm{H}_{34} \mathrm{~F}_{12} \mathrm{NNbO}_{2} \mathrm{Si}_{2}(793.64 \mathrm{~g} / \mathrm{mol})$ : C, 42.38\%; H, 4.32\%; N, 1.76\%. Found: C, $42.28 \%, \mathrm{H}, 4.10 \%, \mathrm{~N}, 1.63 \%$ 
NMR Characterization of $\left[\mathrm{CF}_{3}-\mathrm{ONO}\right] \mathrm{Nb}\left(\mathrm{CH}_{2} \mathrm{Si}\left(\mathrm{CH}_{3}\right)_{3}\right)_{2}(6)$

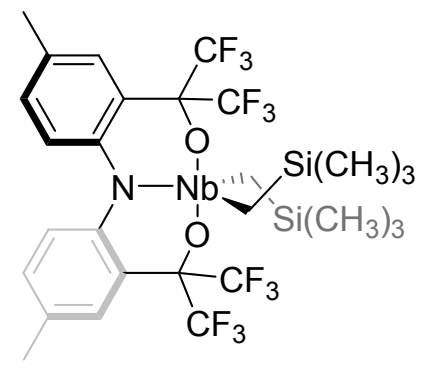

6

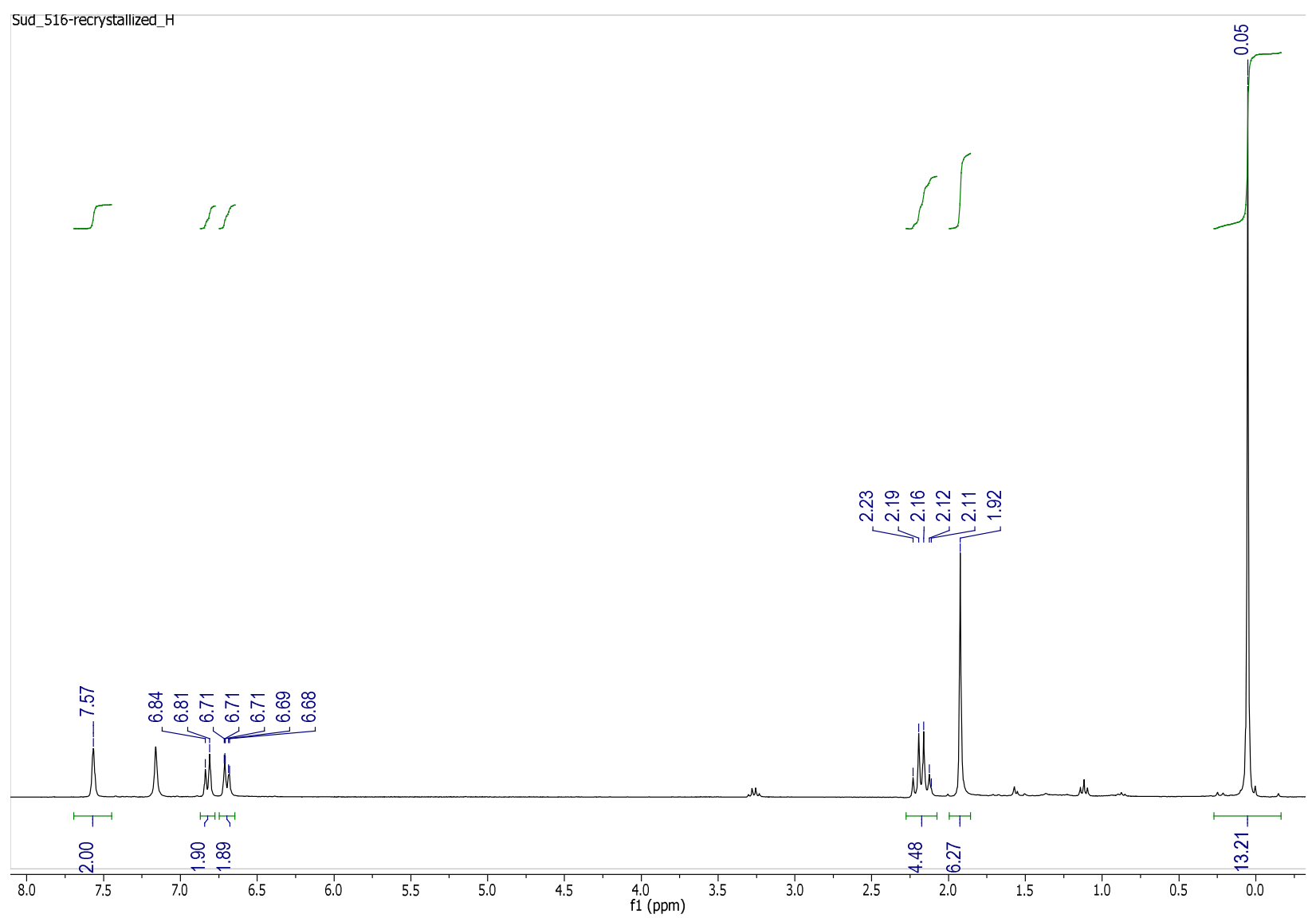

Figure S58. ${ }^{1} \mathrm{H}$ NMR spectrum of $6\left(300 \mathrm{MHz}, \mathrm{C}_{6} \mathrm{D}_{6}\right)$ 


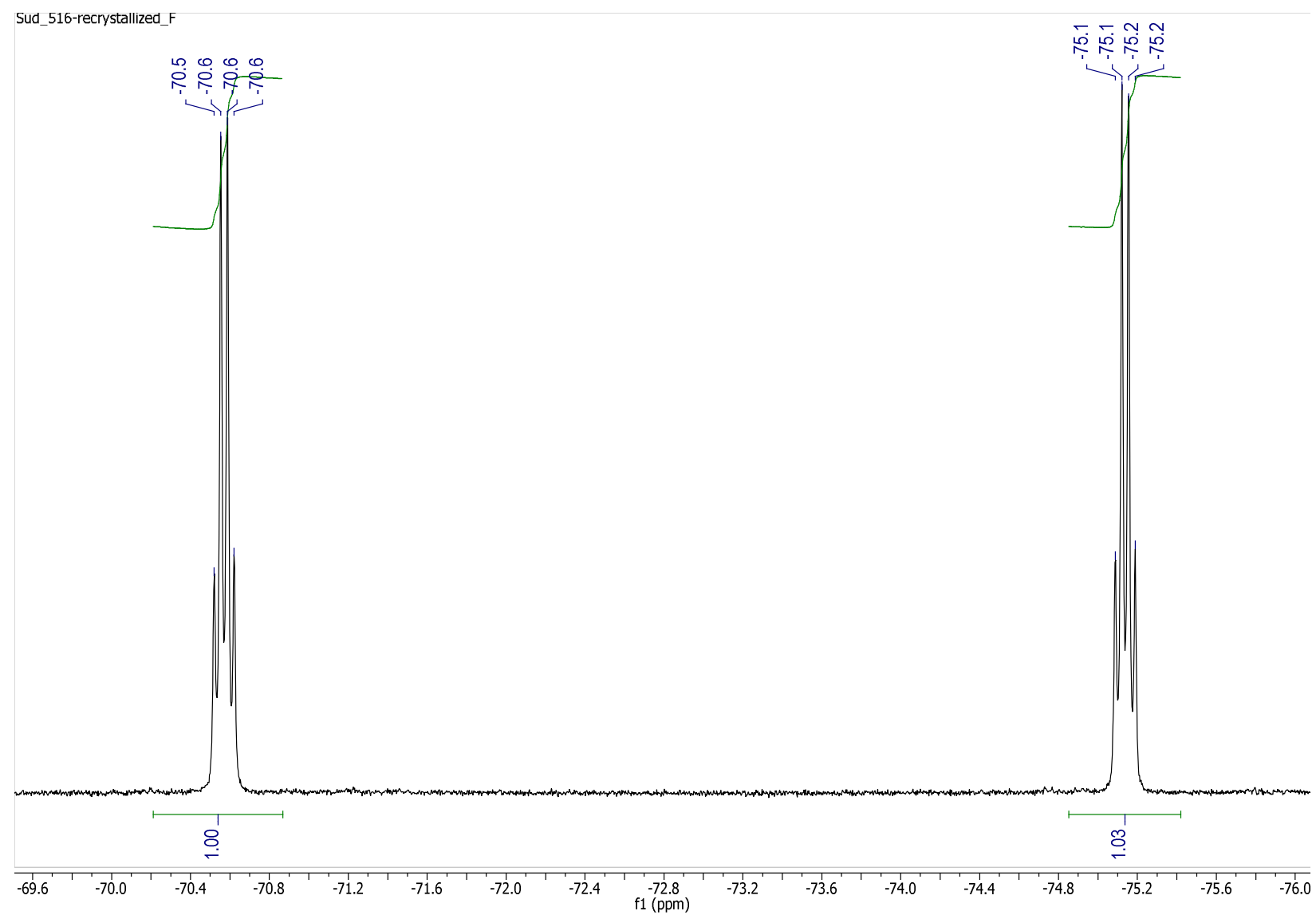

Figure S59. ${ }^{19} \mathrm{~F}$ NMR spectrum of $6\left(\mathrm{C}_{6} \mathrm{D}_{6}, 282 \mathrm{MHz}\right)$ 


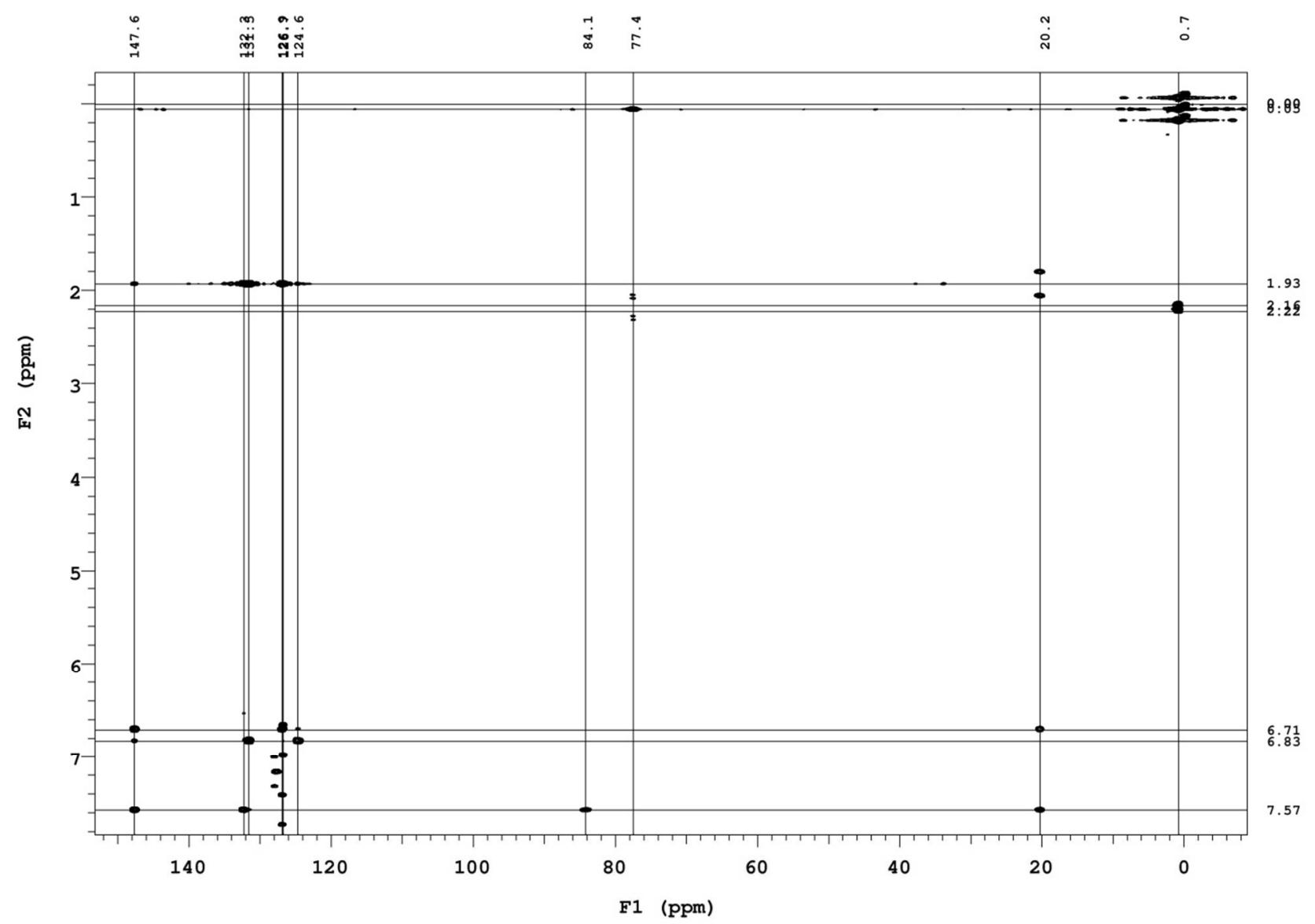

Figure S60. ${ }^{1} \mathrm{H}^{13}{ }^{13} \mathrm{gHMBC}$ spectrum of $6\left(\mathrm{C}_{6} \mathrm{D}_{6}, 500 \mathrm{MHz}\right)$ 


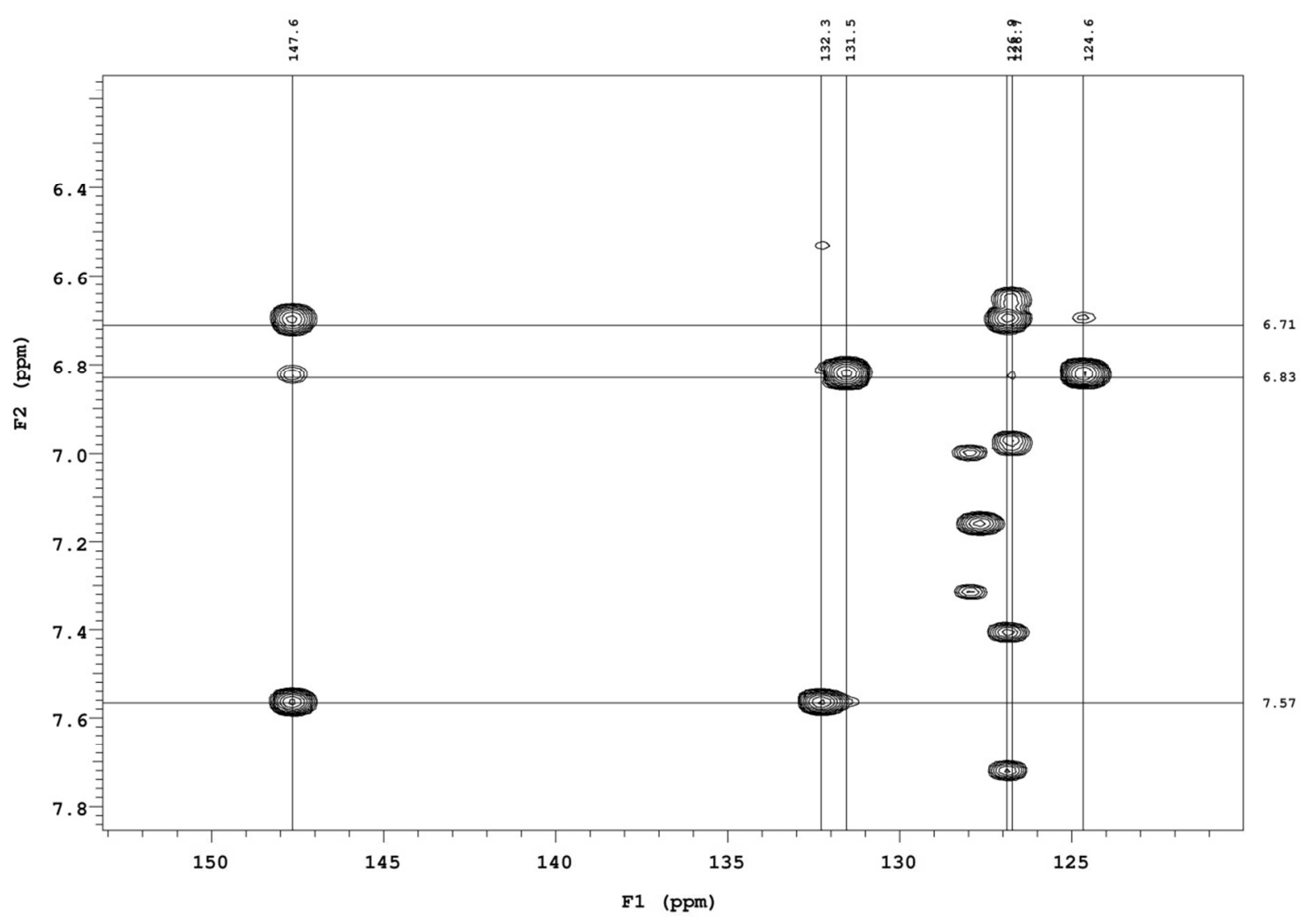

Figure S61. ${ }^{1} \mathrm{H}-{ }^{13} \mathrm{C} g \mathrm{HMBC}$ spectrum of $6-$ Expansion $\left(\mathrm{C}_{6} \mathrm{D}_{6}, 500 \mathrm{MHz}\right)$ 


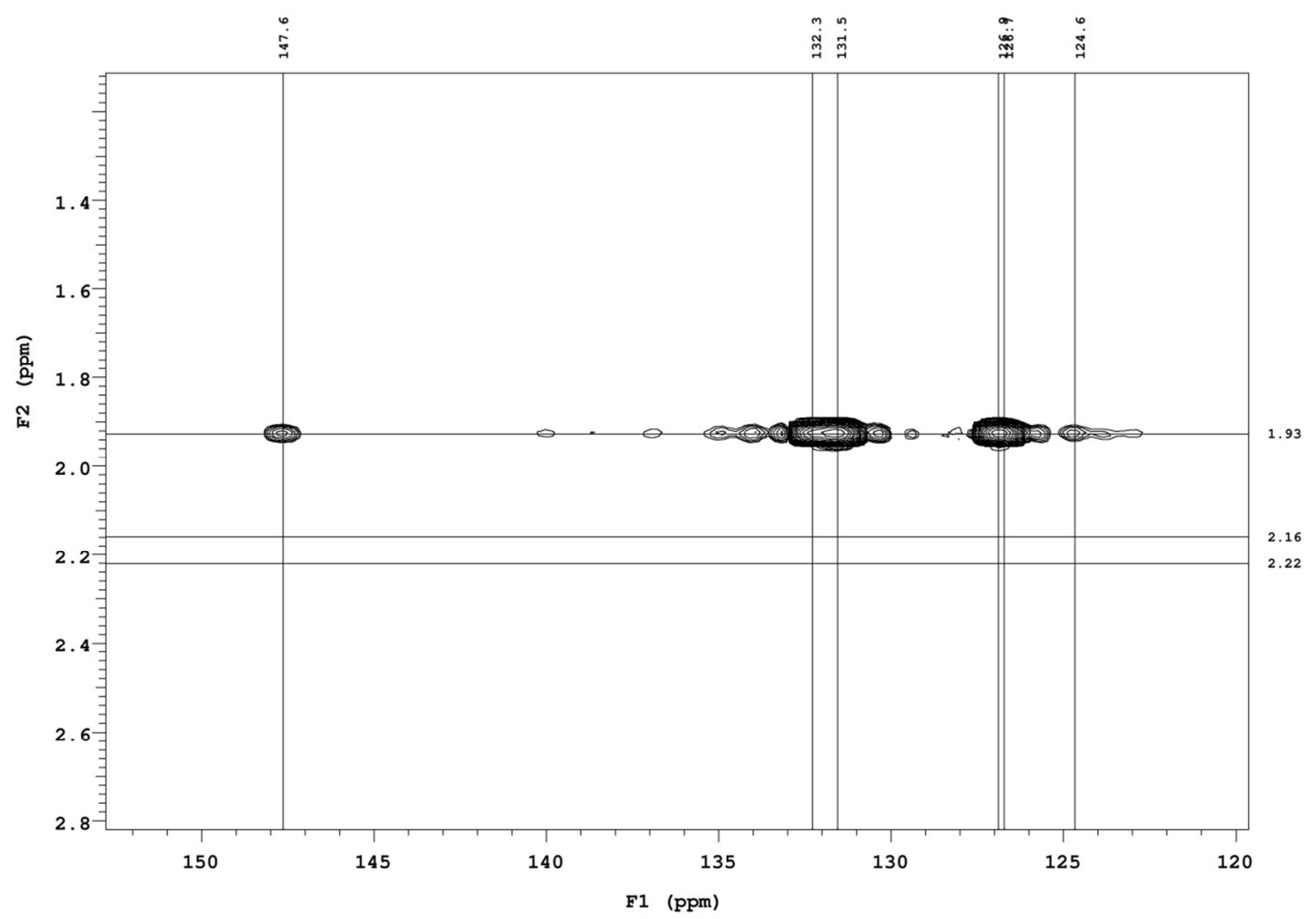

Figure S62. ${ }^{1} \mathrm{H}_{-}{ }^{13} \mathrm{C} g \mathrm{HMBC}$ spectrum of $6-$ Expansion $\left(\mathrm{C}_{6} \mathrm{D}_{6}, 500 \mathrm{MHz}\right)$ 


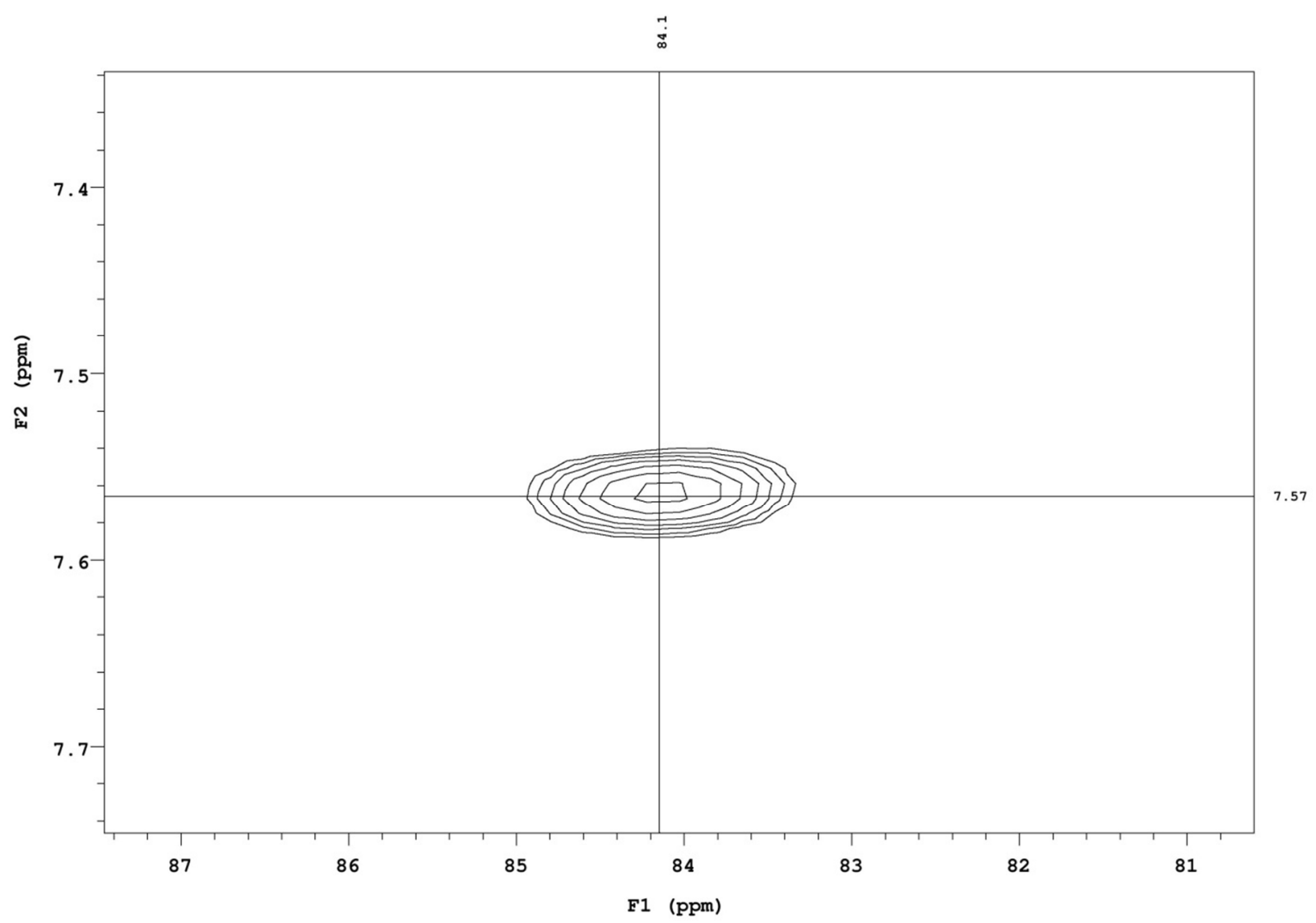

Figure S63. ${ }^{1} \mathrm{H}-{ }^{13} \mathrm{C} g \mathrm{HMBC}$ spectrum of $6-$ Expansion $\left(\mathrm{C}_{6} \mathrm{D}_{6}, 500 \mathrm{MHz}\right)$ 


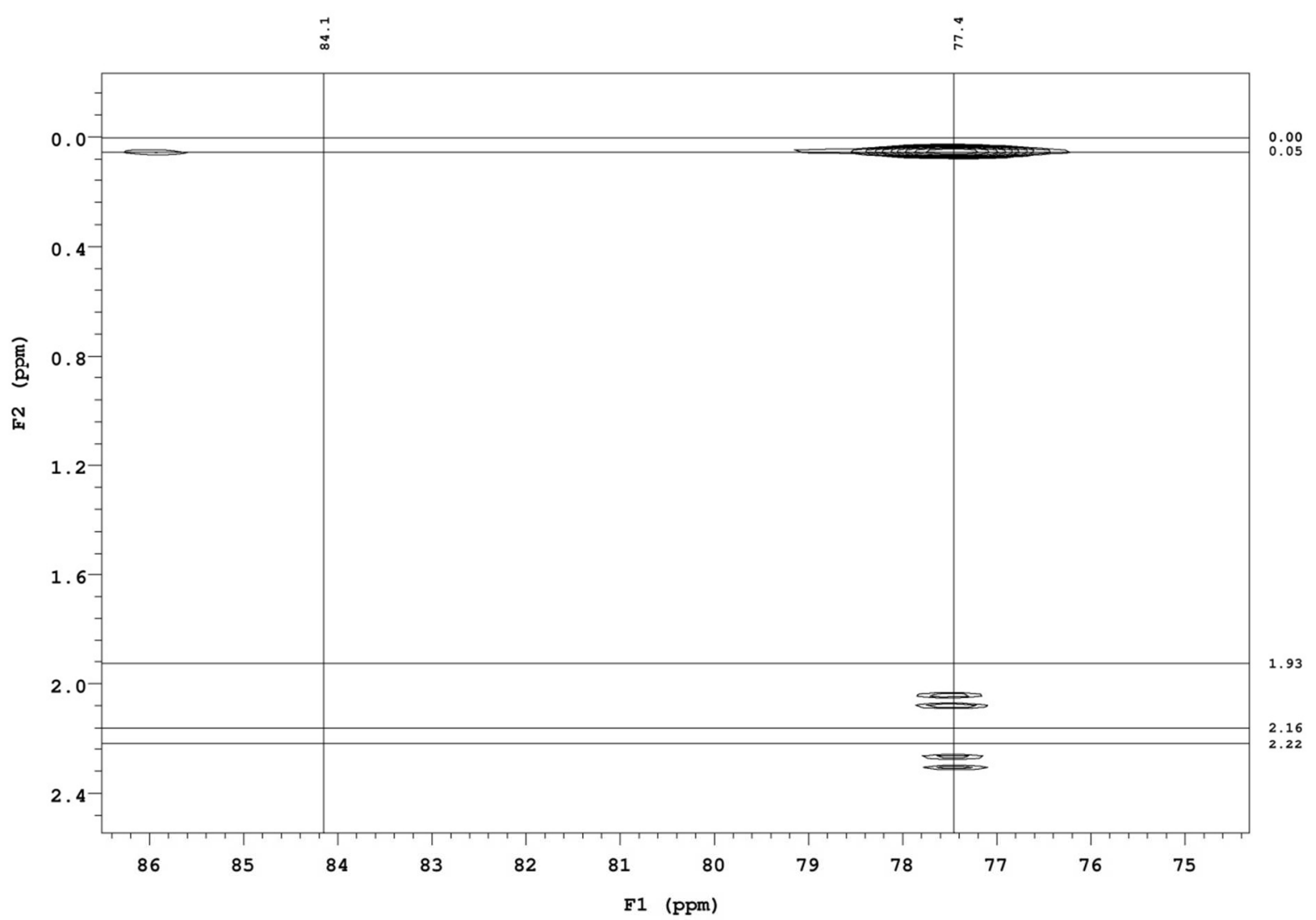

Figure S64. ${ }^{1} \mathrm{H}-{ }^{13} \mathrm{C} g \mathrm{HMBC}$ spectrum of $6-$ Expansion $\left(\mathrm{C}_{6} \mathrm{D}_{6}, 500 \mathrm{MHz}\right)$ 


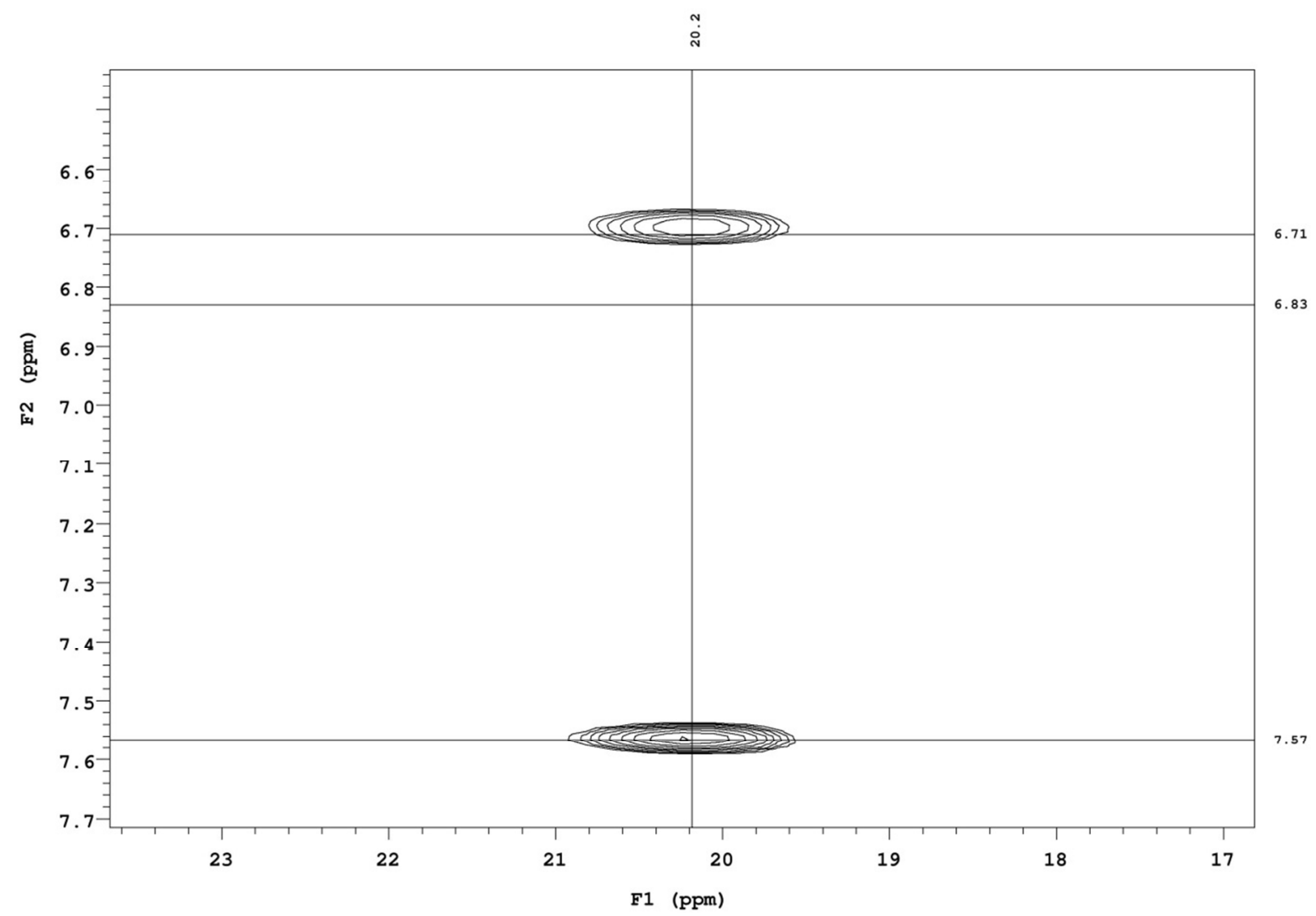

Figure S65. ${ }^{1} \mathrm{H}-{ }^{13} \mathrm{C} g \mathrm{HMBC}$ spectrum of $6-$ Expansion $\left(\mathrm{C}_{6} \mathrm{D}_{6}, 500 \mathrm{MHz}\right)$ 


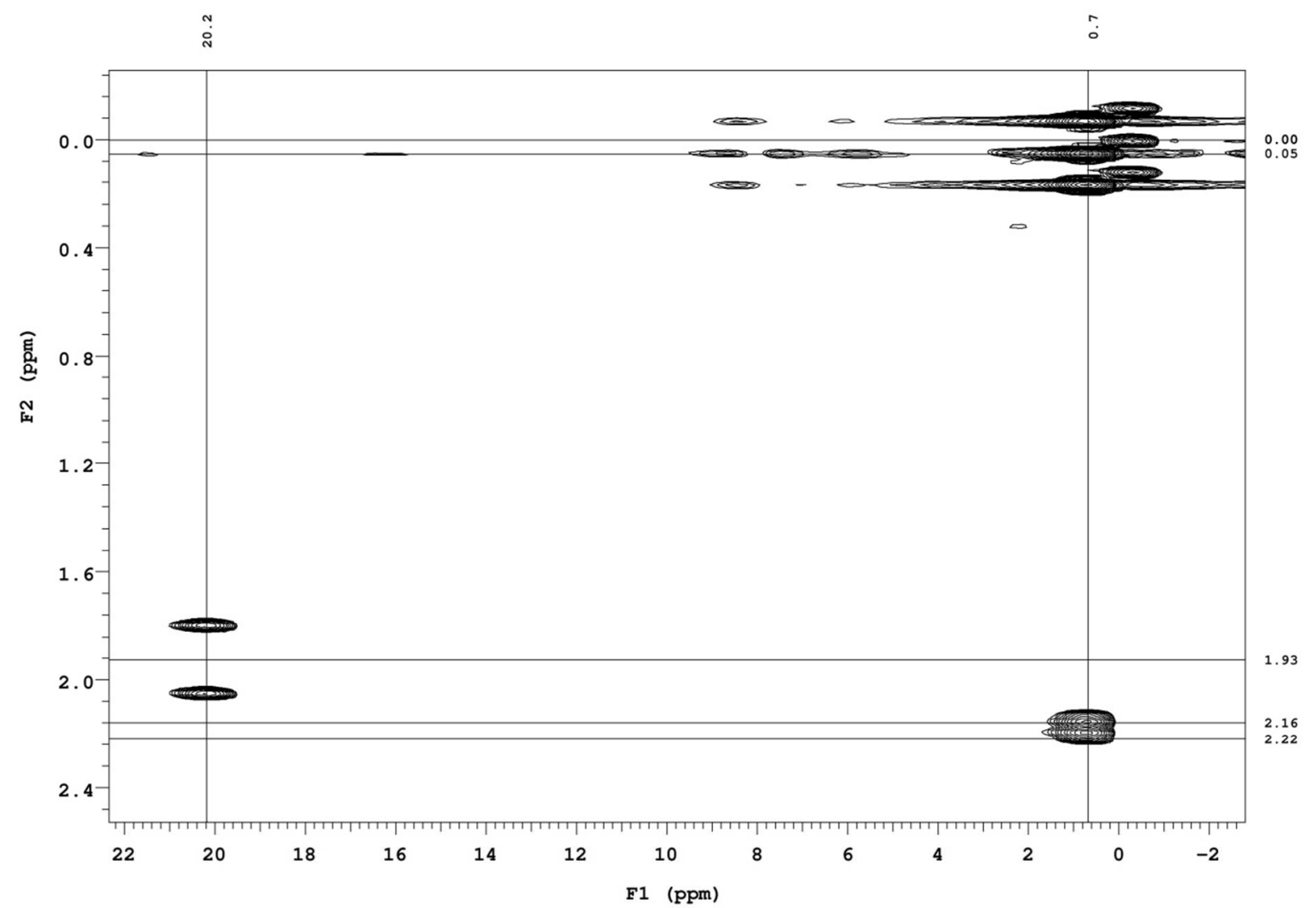

Figure S66. ${ }^{1} \mathrm{H}-{ }^{13} \mathrm{C} g \mathrm{HMBC}$ spectrum of $6-$ Expansion $\left(\mathrm{C}_{6} \mathrm{D}_{6}, 500 \mathrm{MHz}\right)$ 


\section{Synthesis of $\left[\mathrm{CF}_{3}-\mathrm{ONO}\right] \mathrm{Nb}\left(\mathrm{CH}_{2} \mathrm{C}\left(\mathrm{CH}_{3}\right)_{2}\left(\mathrm{C}_{6} \mathrm{H}_{5}\right)\right)_{2}(7)$}

In a vial, 3-OEt $2(0.146 \mathrm{~g}, 0.191 \mathrm{mmol})$ was dissolved in $2 \mathrm{~mL}$ pentane and chilled to $-35{ }^{\circ} \mathrm{C}$ for ca. 30 min. 2-Methyl-2-phenylpropylmagnesium chloride solution $(0.382 \mu \mathrm{L}, 2$ equiv) was added to the cold solution of $\mathbf{3}-\mathbf{E t}_{2} \mathbf{O}$ in drops using a micropipette. The solution immediately turned color to orange with copious amounts of a precipitate. With passage of time, the orange color intensified and persisted. The reaction mixture was stirred for $4 \mathrm{~h}$ and then filtered through a pad of Celite. All volatiles were removed in vacuo to give a tacky residue. Approximately 1 $\mathrm{mL}$ of acetonitrile was added to the tacky residue and quickly evaporated to give $\mathbf{7}$ as an orange powder. The product was recrystallized from a pentane-diethyl ether mixture. (Yield $=0.140 \mathrm{~g}$, $82.7 \%)$

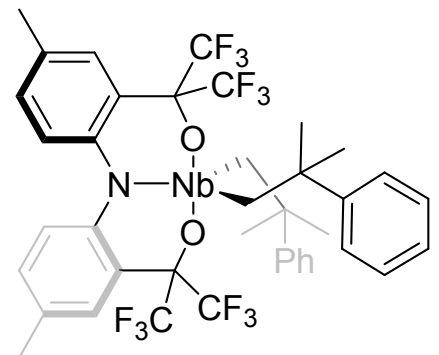

7

${ }^{1} \mathrm{H}$ NMR $\left(500 \mathrm{MHz}, \mathrm{C}_{6} \mathrm{D}_{6}\right) \delta 7.56(\mathrm{~s}, 2 \mathrm{H}, \mathrm{Ar}-H), 7.26-7.20(\mathrm{~m}, 4 \mathrm{H}, \mathrm{Ar}-H), 7.15-7.09(\mathrm{~m}, 4 \mathrm{H}$, Ar- $H), 7.02-6.97(\mathrm{~m}, 2 \mathrm{H}, \mathrm{Ar}-H), 6.81\left(\mathrm{~d}, 2 \mathrm{H},{ }^{3} J_{\mathrm{HH}}=8.5 \mathrm{~Hz}, \mathrm{Ar}-H\right), 6.71\left(\mathrm{dd}, 2 \mathrm{H},{ }^{3} J_{\mathrm{HH}}=8.5\right.$, $\left.{ }^{4} J_{\mathrm{HH}}=1.0 \mathrm{~Hz}, \mathrm{Ar}-H\right), 2.38\left(\mathrm{~d}, 2 \mathrm{H},{ }^{2} J_{\mathrm{HH}}=11.6 \mathrm{~Hz},-\mathrm{CH}_{2} \mathrm{C}\left(\mathrm{CH}_{3}\right)_{2}\left(\mathrm{C}_{6} \mathrm{H}_{5}\right)\right), 2.11\left(\mathrm{~d}, 2 \mathrm{H},{ }^{2} J_{\mathrm{HH}}=\right.$ $\left.11.6 \mathrm{~Hz},-\mathrm{CH}_{2} \mathrm{C}\left(\mathrm{CH}_{3}\right)_{2}\left(\mathrm{C}_{6} \mathrm{H}_{5}\right)\right), 1.94\left(\mathrm{~s}, 6 \mathrm{H}, \mathrm{Ar}-\mathrm{CH}_{3}\right), 1.31\left(\mathrm{~s}, 6 \mathrm{H},-\mathrm{CH}_{2} \mathrm{C}\left(\mathrm{CH}_{3}\right)_{2}\left(\mathrm{C}_{6} \mathrm{H}_{5}\right)\right), 1.20$ (s, $\left.6 \mathrm{H},-\mathrm{CH}_{2} \mathrm{C}\left(\mathrm{CH}_{3}\right)_{2}\left(\mathrm{C}_{6} \mathrm{H}_{5}\right)\right)$ ppm.

${ }^{13} \mathrm{C}\left\{{ }^{1} \mathrm{H}\right\}$ NMR (126 MHz, $\left.\mathrm{C}_{6} \mathrm{D}_{6}\right) \delta 150.0(\mathrm{Ar}-\mathrm{C}), 147.8(\mathrm{Ar}-\mathrm{C}), 132.3(\mathrm{Ar}-\mathrm{C}), 131.6(\mathrm{Ar}-\mathrm{C})$, $128.4(\mathrm{Ar}-C), 126.9(\mathrm{Ar}-C), 126.9(\mathrm{Ar}-C), 126.5(\mathrm{Ar}-C), 126.0(\mathrm{Ar}-C), 125.5(\mathrm{Ar}-C), 124.9(\mathrm{Ar}-$ C), $\quad 99.1 \quad\left(-\mathrm{CH}_{2} \mathrm{C}\left(\mathrm{CH}_{3}\right)_{2}\left(\mathrm{C}_{6} \mathrm{H}_{5}\right)\right), \quad 84.1 \quad(\mathrm{Ar}-\mathrm{C}), \quad 41.9 \quad\left(-\mathrm{CH}_{2} \mathrm{C}\left(\mathrm{CH}_{3}\right)_{2}\left(\mathrm{C}_{6} \mathrm{H}_{5}\right)\right), \quad 32.0$ $\left(-\mathrm{CH}_{2} \mathrm{C}\left(\mathrm{CH}_{3}\right)_{2}\left(\mathrm{C}_{6} \mathrm{H}_{5}\right)\right), 31.8\left(-\mathrm{CH}_{2} \mathrm{C}\left(\mathrm{CH}_{3}\right)_{2}\left(\mathrm{C}_{6} \mathrm{H}_{5}\right)\right), 20.2\left(\mathrm{Ar}-\mathrm{CH}_{3}\right) \mathrm{ppm}$. 
${ }^{19} \mathrm{~F} \mathrm{NMR}\left(282 \mathrm{MHz}, \mathrm{C}_{6} \mathrm{D}_{6}\right) \delta-69.8\left(\mathrm{q}, 6 \mathrm{~F},{ }^{4} J_{\mathrm{FF}}=9.8 \mathrm{~Hz}\right),-74.4\left(\mathrm{q}, 6 \mathrm{~F},{ }^{4} J_{\mathrm{FF}}=9.9 \mathrm{~Hz}\right) \mathrm{ppm}$.

Anal. Calcd. for $\mathrm{C}_{40} \mathrm{H}_{38} \mathrm{~F}_{12} \mathrm{NO}_{2} \mathrm{Nb}$ : C, 54.25\%; H, 4.33\%; N, 1.58\%. Found: C, 53.77\%; H, $4.29 \% ; \mathrm{N}, 1.61 \%$. 
NMR Characterization of $\left[\mathrm{CF}_{3}-\mathrm{ONO}\right] \mathrm{Nb}\left(\mathrm{CH}_{2} \mathrm{C}\left(\mathrm{CH}_{3}\right)_{2}\left(\mathrm{C}_{6} \mathrm{H}_{5}\right)\right)_{2}(7)$

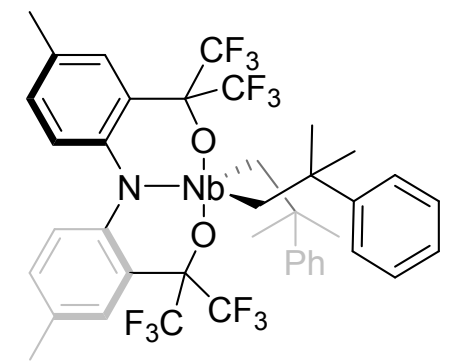

7

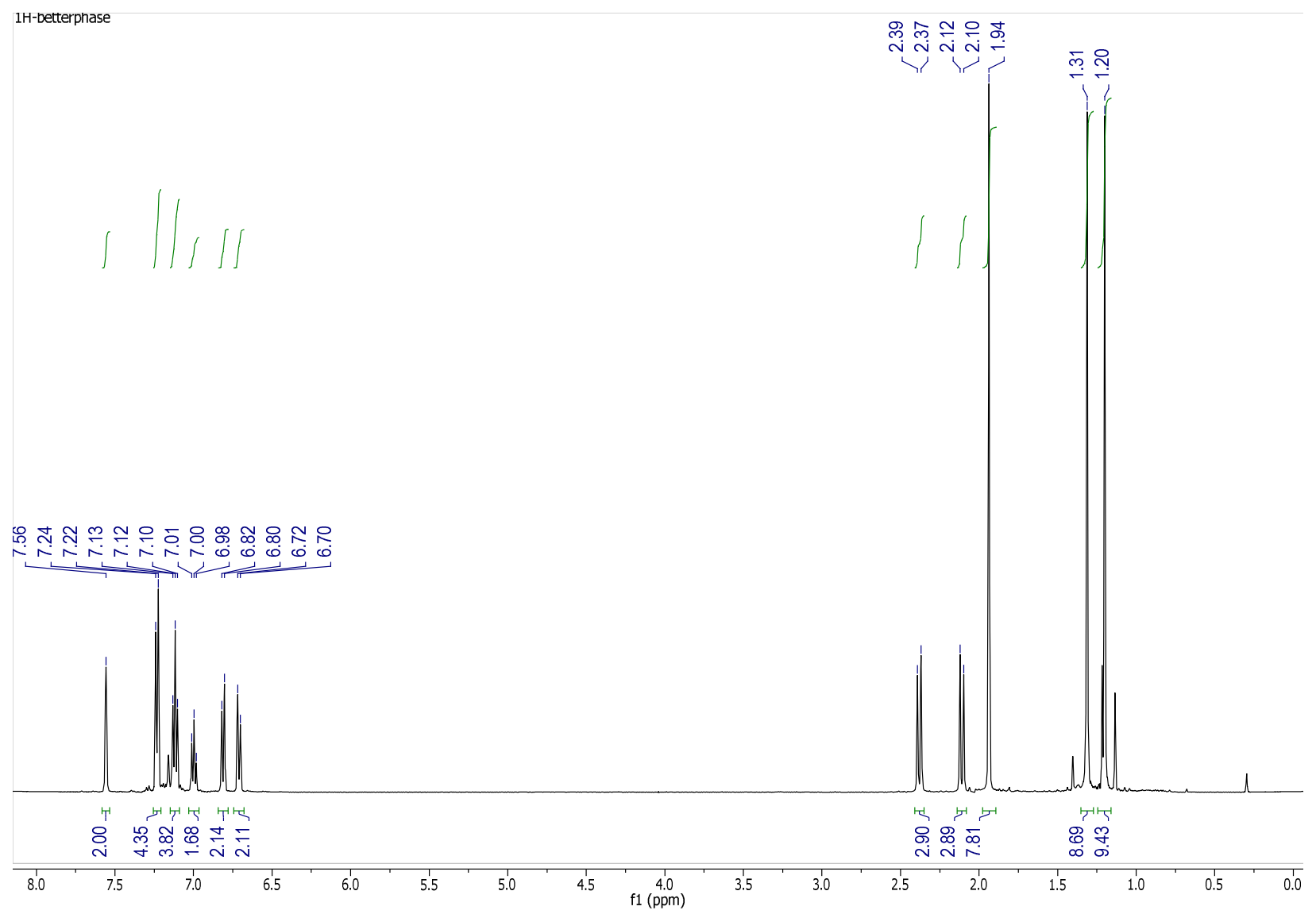

Figure S67. ${ }^{1} \mathrm{H}$ NMR spectrum of $7\left(\mathrm{C}_{6} \mathrm{D}_{6}, 500 \mathrm{MHz}\right)$ 


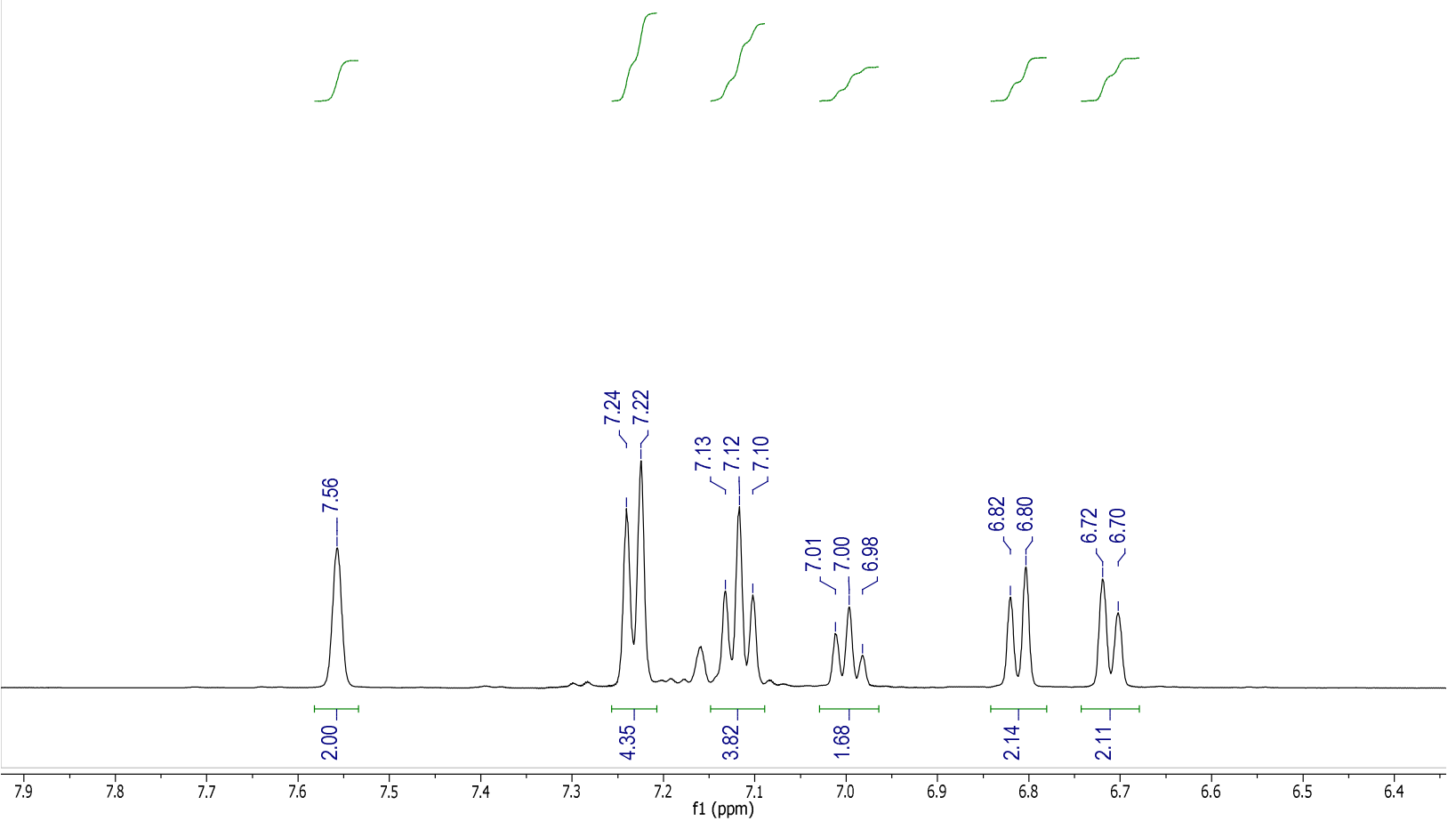

Figure S68. ${ }^{1} \mathrm{H}$ NMR spectrum of 7 - Expansion $\left(\mathrm{C}_{6} \mathrm{D}_{6}, 500 \mathrm{MHz}\right)$ 


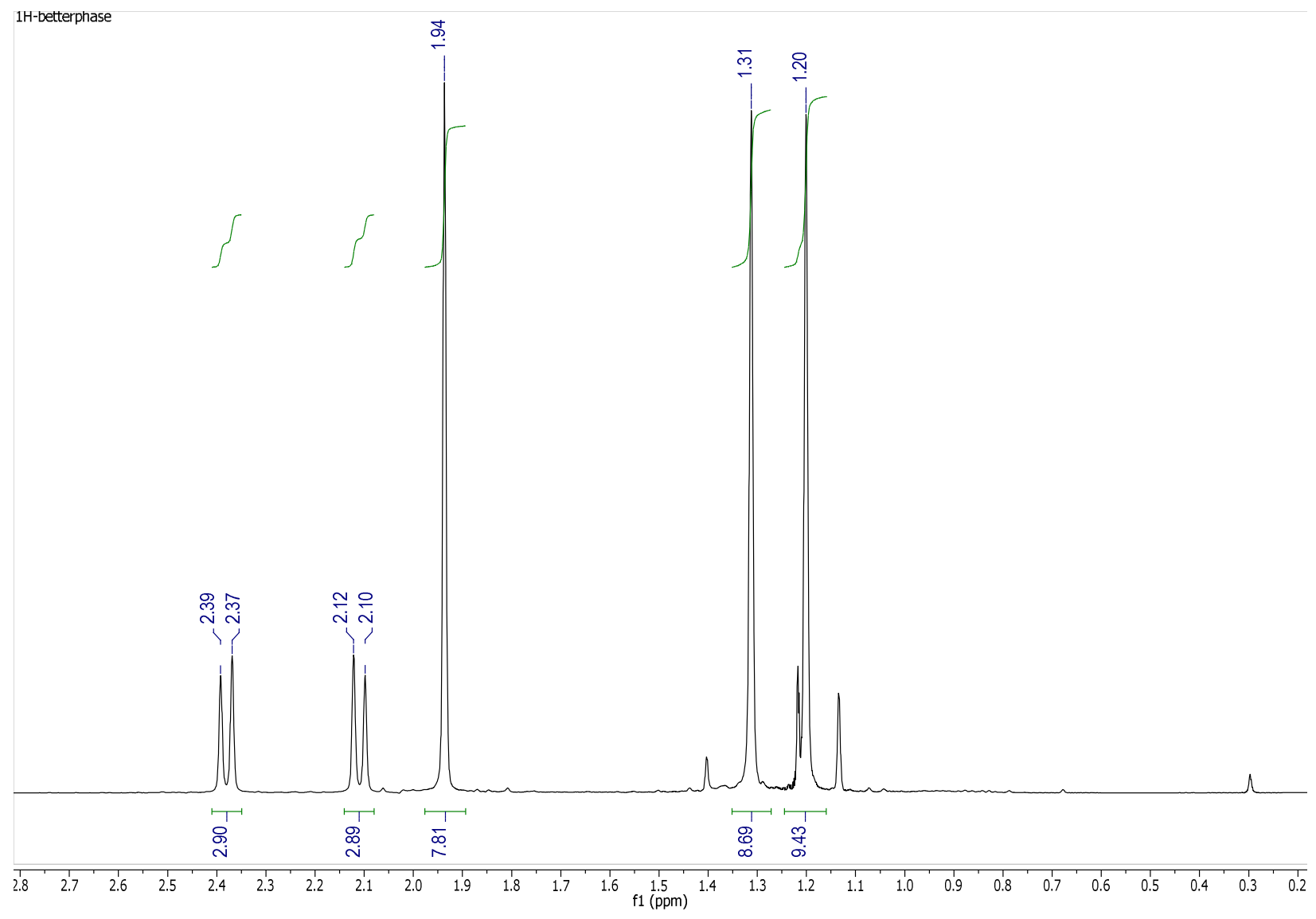

Figure S69. ${ }^{1} \mathrm{H}$ NMR spectrum of 7 - Expansion $\left(\mathrm{C}_{6} \mathrm{D}_{6}, 500 \mathrm{MHz}\right)$ 


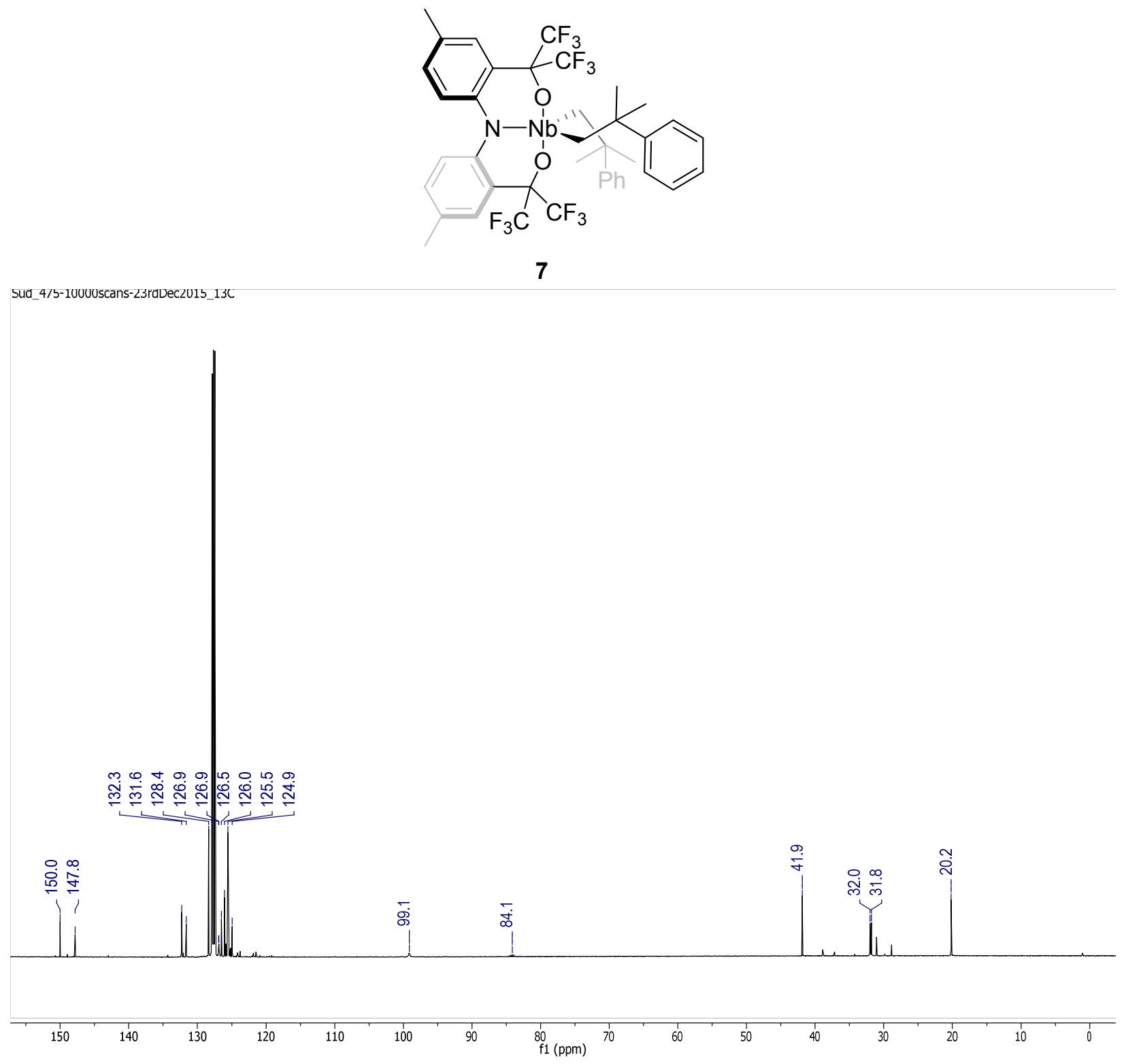

Figure S70. ${ }^{13} \mathrm{C}\left\{{ }^{1} \mathrm{H}\right\}$ NMR spectrum of $7\left(\mathrm{C}_{6} \mathrm{D}_{6}, 126 \mathrm{MHz}\right)$

S83 


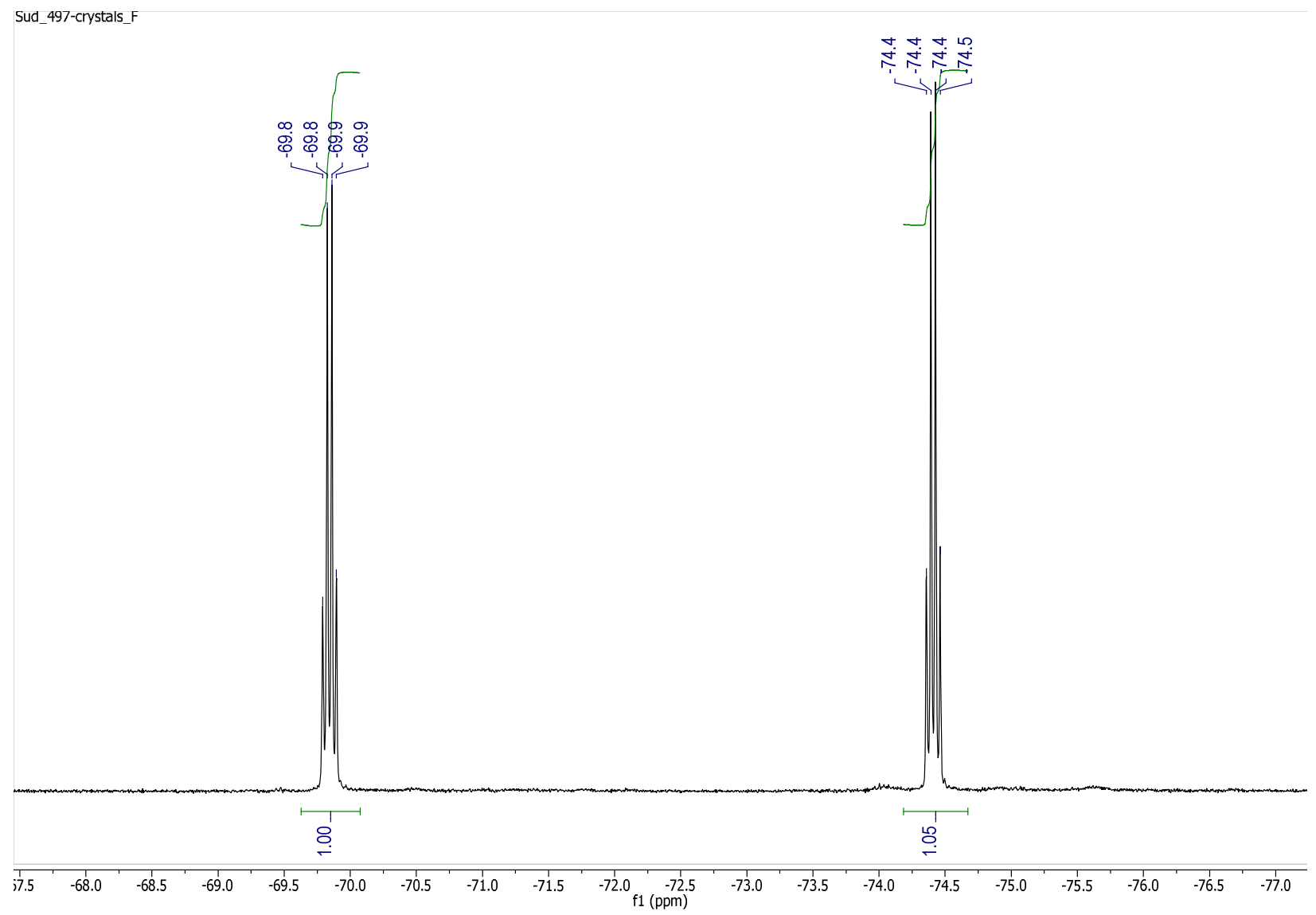

Figure S71. ${ }^{19} \mathrm{~F}$ NMR spectrum of $7\left(\mathrm{C}_{6} \mathrm{D}_{6}, 282 \mathrm{MHz}\right)$ 


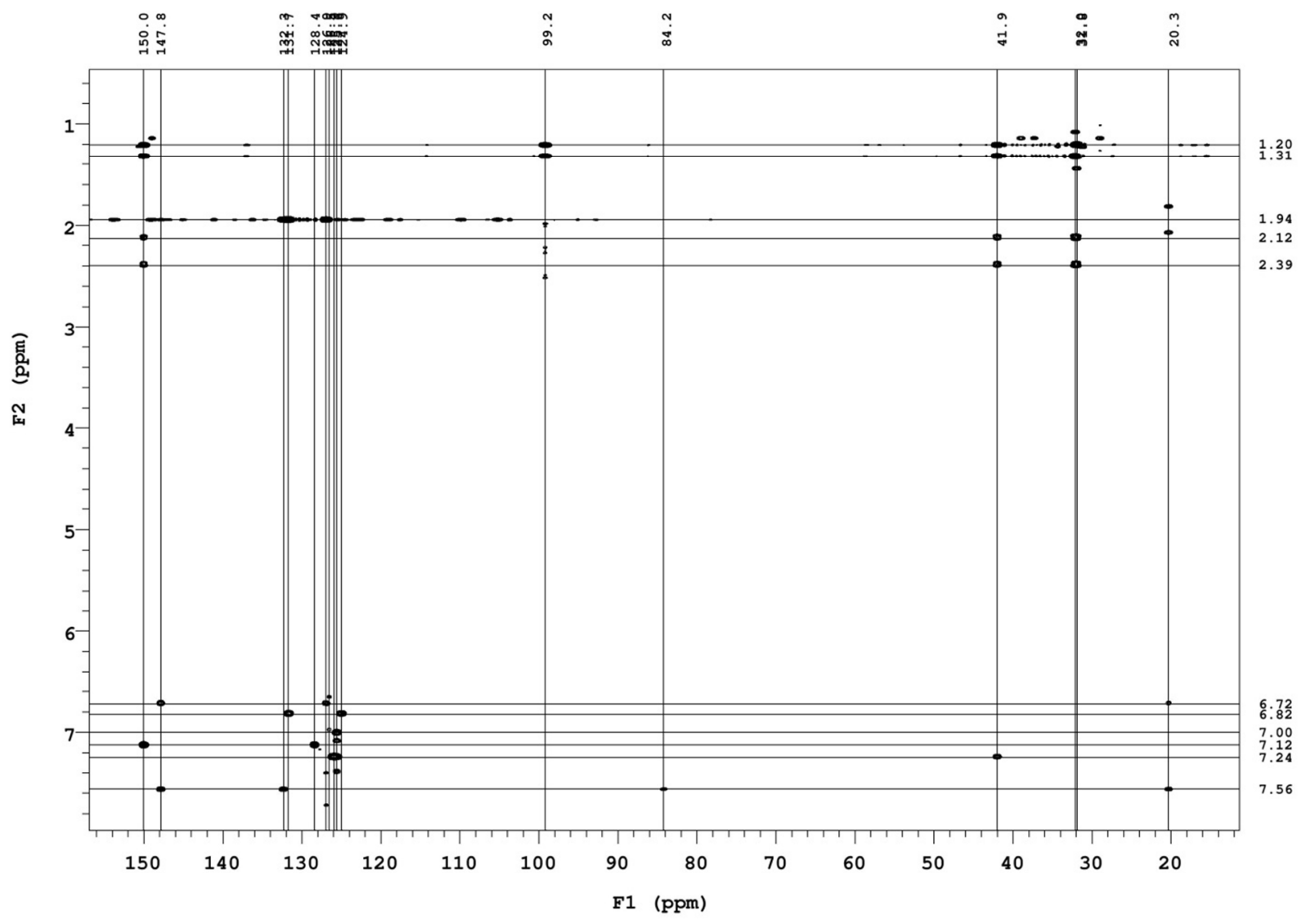

Figure S72. ${ }^{1} \mathrm{H}-{ }^{13} \mathrm{C}$ gHMBC spectrum of 7 (500 MHz, $\left.\mathrm{C}_{6} \mathrm{D}_{6}\right)$ 


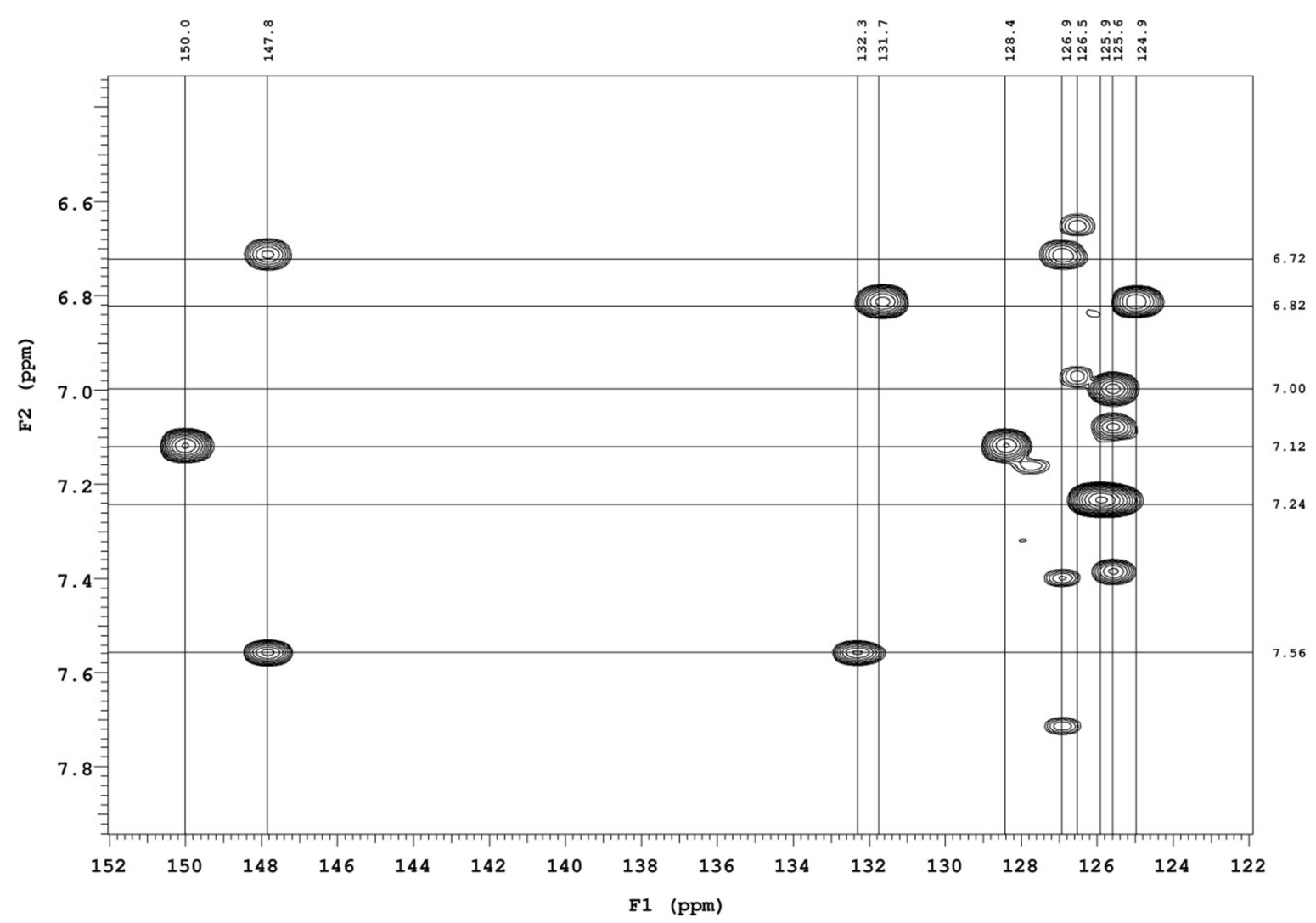

Figure S73. ${ }^{1} \mathrm{H}-{ }^{13} \mathrm{C}$ gHMBC spectrum of 7 - Expansion $\left(500 \mathrm{MHz}, \mathrm{C}_{6} \mathrm{D}_{6}\right)$ 


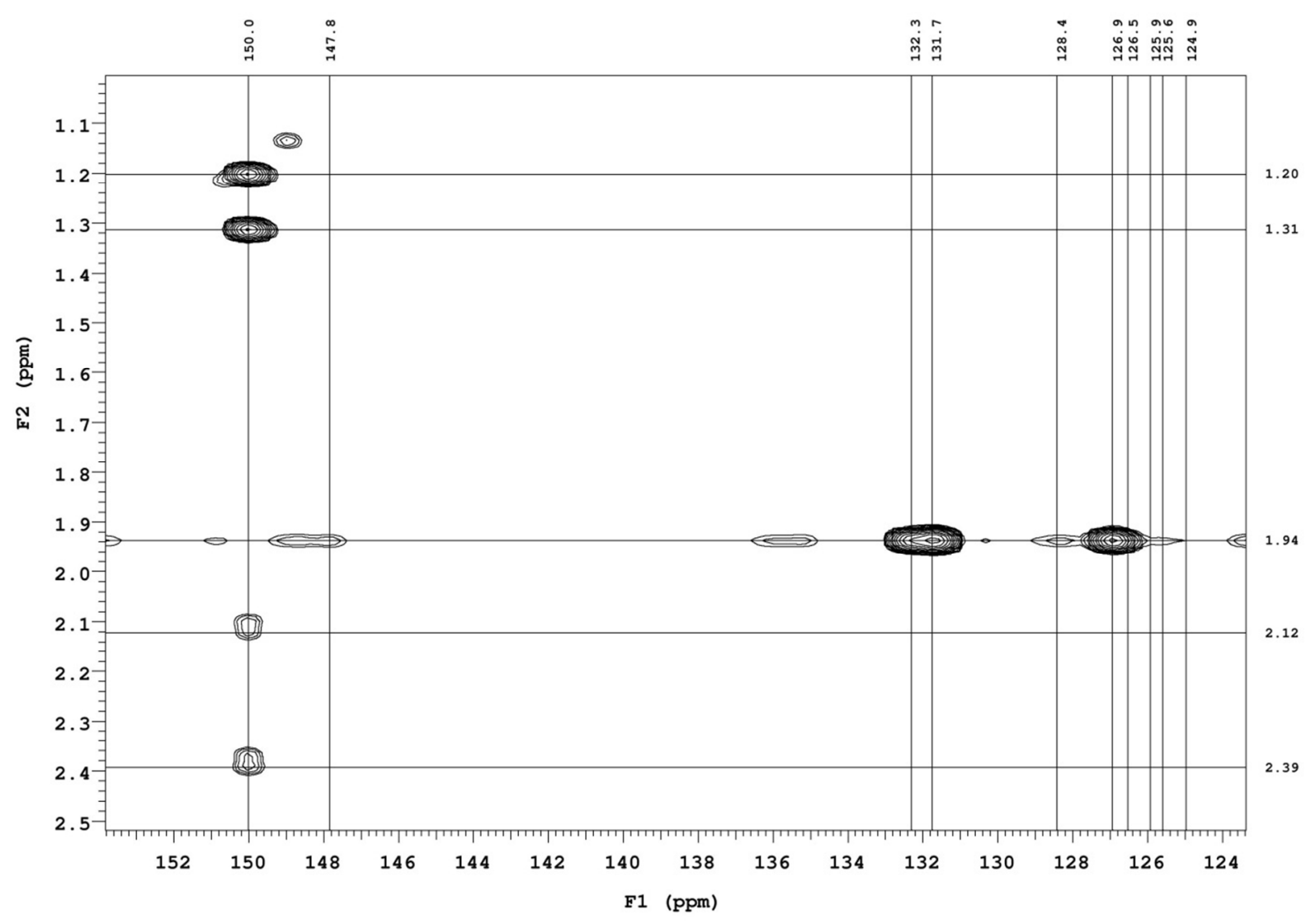

Figure S74. ${ }^{1} \mathrm{H}-{ }^{13} \mathrm{C}$ gHMBC spectrum of 7 - Expansion $\left(500 \mathrm{MHz}, \mathrm{C}_{6} \mathrm{D}_{6}\right)$ 


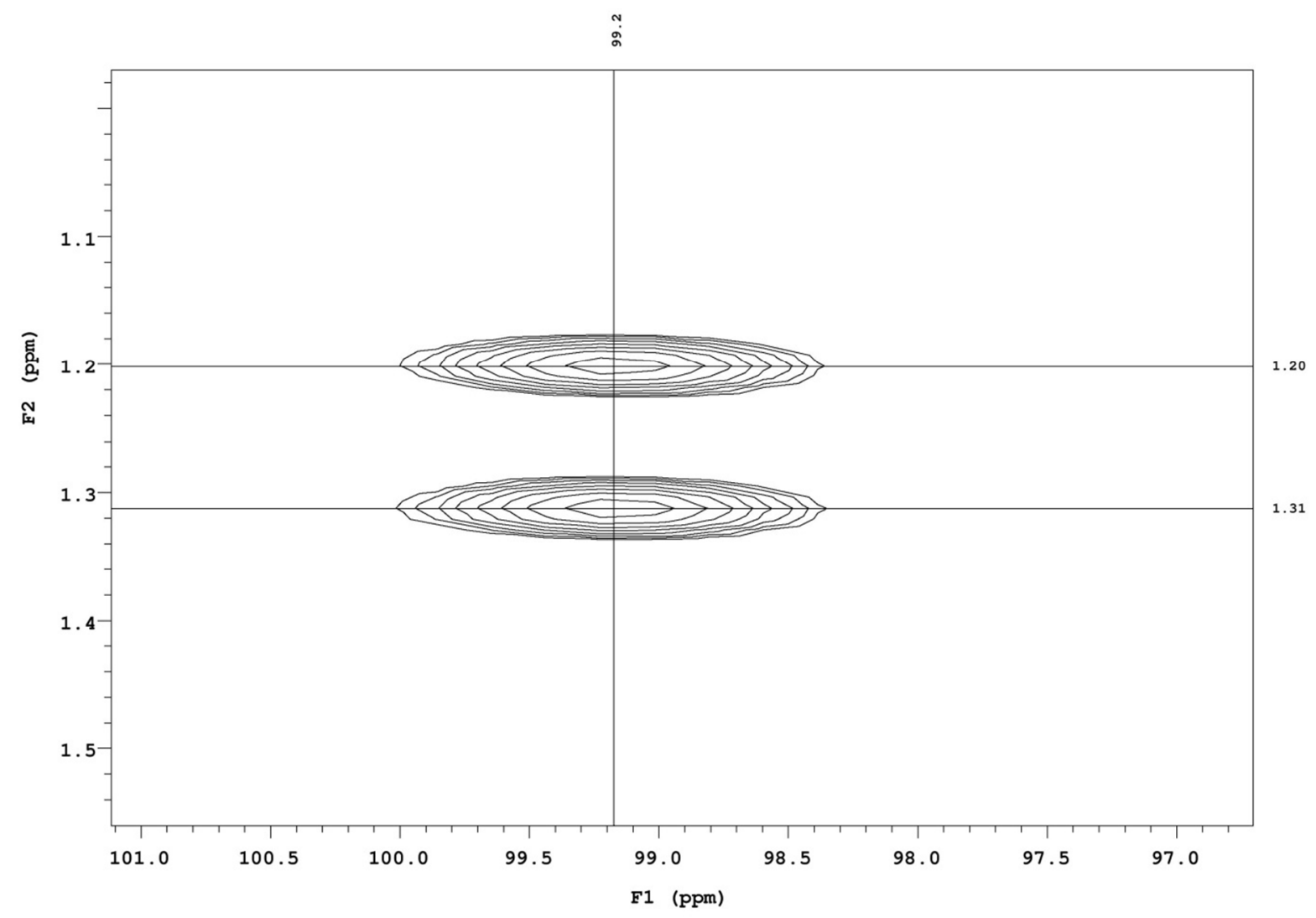

Figure S75. ${ }^{1} \mathrm{H}-{ }^{13} \mathrm{C}$ gHMBC spectrum of 7 - Expansion $\left(500 \mathrm{MHz}, \mathrm{C}_{6} \mathrm{D}_{6}\right)$ 


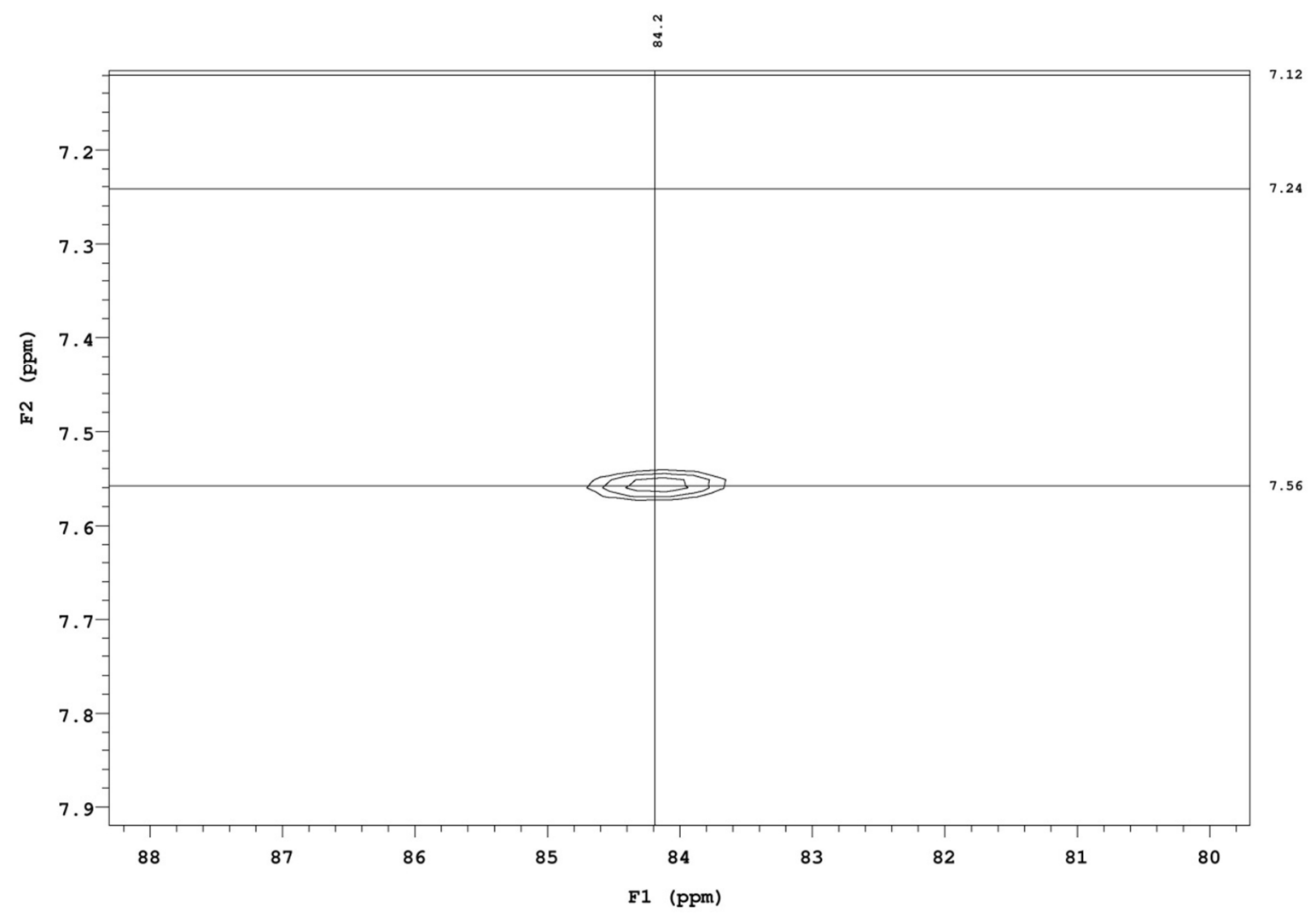

Figure S76. ${ }^{1} \mathrm{H}-{ }^{13} \mathrm{C}$ gHMBC spectrum of 7 - Expansion $\left(500 \mathrm{MHz}, \mathrm{C}_{6} \mathrm{D}_{6}\right)$ 


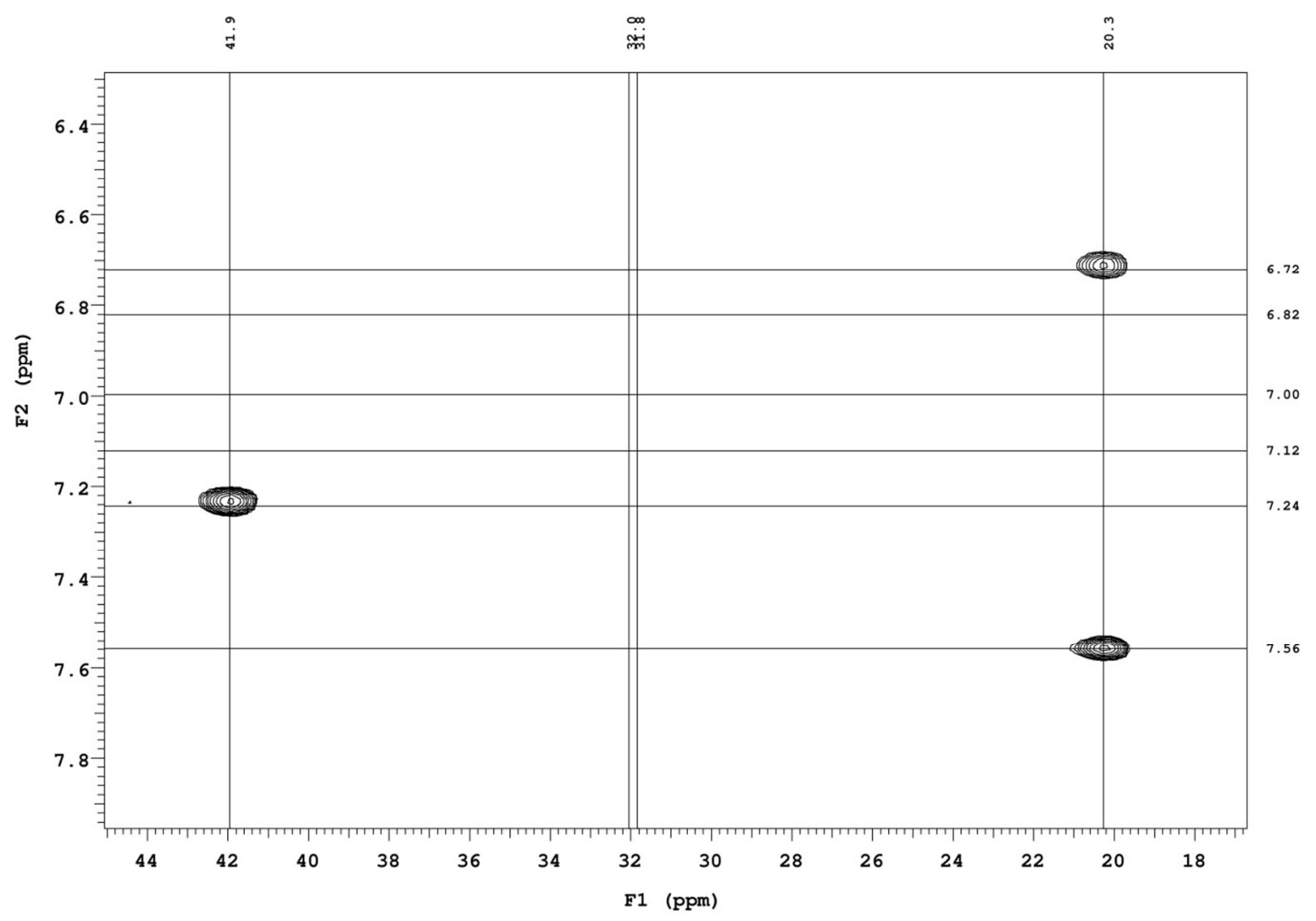

Figure S77. ${ }^{1} \mathrm{H}-{ }^{13} \mathrm{C}$ gHMBC spectrum of 7 - Expansion $\left(500 \mathrm{MHz}, \mathrm{C}_{6} \mathrm{D}_{6}\right)$ 


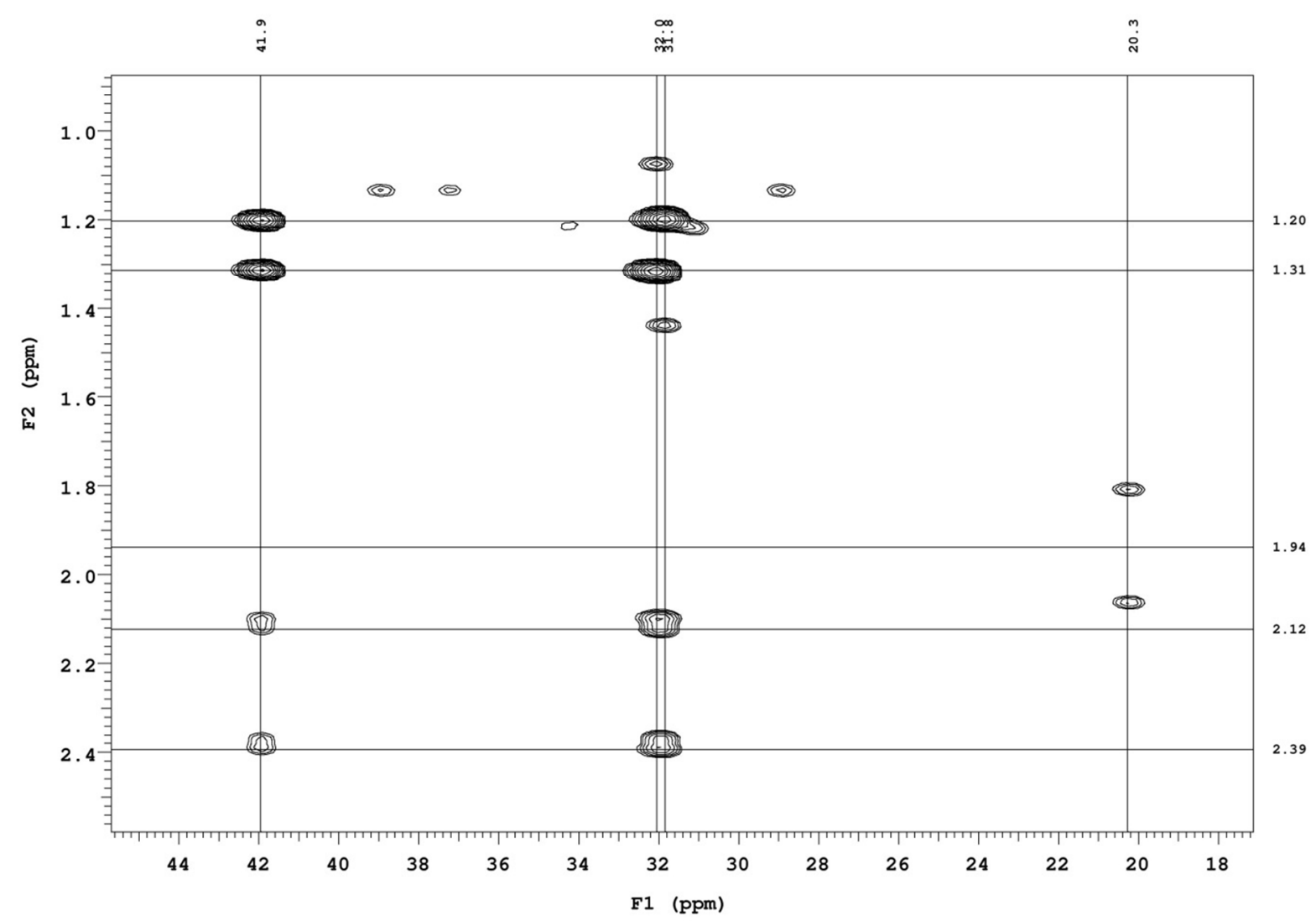

Figure S78. ${ }^{1} \mathrm{H}-{ }^{13} \mathrm{C}$ gHMBC spectrum of 7 - Expansion $\left(500 \mathrm{MHz}, \mathrm{C}_{6} \mathrm{D}_{6}\right)$ 


\section{Synthesis of $\left[\mathrm{CF}_{3}-\mathrm{ONO}\right] \mathrm{Ta}\left(\mathrm{CH}_{2} \mathrm{C}\left(\mathrm{CH}_{3}\right)_{2}\left(\mathrm{C}_{6} \mathrm{H}_{5}\right)\right)(9)$}

In a vial, complex $8(0.221 \mathrm{~g}, 0.260 \mathrm{mmol})$ was dissolved in $2 \mathrm{~mL}$ of pentane and chilled to -35 ${ }^{\circ} \mathrm{C}$. To the cold solution of $\mathbf{8}, 2$-methyl-2-phenylpropylmagnesium chloride solution (1.04 mL, $0.520 \mathrm{mmol}, 2$ equiv) was added in drops using a micropipette. The reaction mixture turned orange in approx. $5 \mathrm{~min}$ with copious amounts of a precipitate. After $4 \mathrm{~h}$ of stirring, the reaction mixture was filtered to remove the salt by-product and then all volatiles were removed provide a yellow oil. $3 \mathrm{~mL}$ of acetonitrile was added to the yellow oil and allowed to sit for a few minutes, after which time it was removed in vacuo to give $\mathbf{9}$ as a fine yellow powder. The product was recrystallized from pentane. (Yield: $0.175 \mathrm{~g}, 69.1 \%$ )

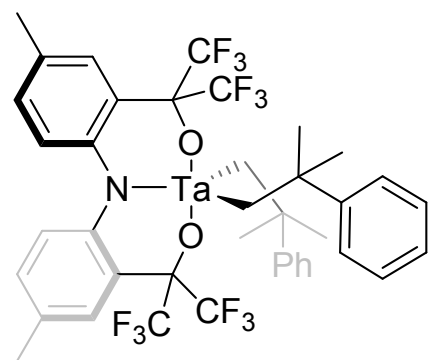

9

${ }^{1} \mathrm{H}$ NMR (500 MHz, $\left.\mathrm{C}_{6} \mathrm{D}_{6}\right) \delta 7.54(\mathrm{~s}, 2 \mathrm{H}, \mathrm{Ar}-H), 7.26-7.20(\mathrm{~m}, 4 \mathrm{H} \mathrm{Ar}-H), 7.11\left(\mathrm{t}, 4 \mathrm{H},{ }^{3} J_{\mathrm{HH}}=\right.$ $7.7 \mathrm{~Hz}, \operatorname{Ar}-H), 6.99\left(\mathrm{t}, 2 \mathrm{H},{ }^{3} J_{\mathrm{HH}}=7.3 \mathrm{~Hz}, \operatorname{Ar}-H\right), 6.76\left(\mathrm{~d}, 2 \mathrm{H},{ }^{3} J_{\mathrm{HH}}=8.5 \mathrm{~Hz}, \operatorname{Ar}-H\right), 6.71(\mathrm{dd}$, $\left.2 \mathrm{H},{ }^{3} J_{\mathrm{HH}}=8.5,{ }^{4} J_{\mathrm{HH}}=1.9 \mathrm{~Hz}, \mathrm{Ar}-H\right), 1.94\left(\mathrm{~s}, 6 \mathrm{H}, \mathrm{Ar}-\mathrm{CH}_{3}\right), 1.91\left(\mathrm{~d},{ }^{2} J_{\mathrm{HH}}=12.7 \mathrm{~Hz}, 2 \mathrm{H}\right.$,

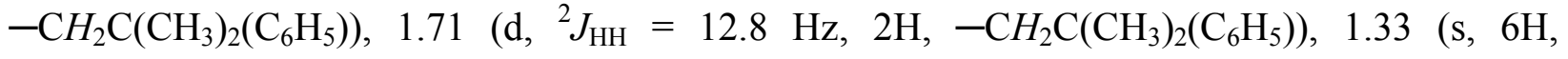
$\left.-\mathrm{CH}_{2} \mathrm{C}\left(\mathrm{CH}_{3}\right)_{2}\left(\mathrm{C}_{6} \mathrm{H}_{5}\right)\right), 1.24\left(\mathrm{~s}, 6 \mathrm{H},-\mathrm{CH}_{2} \mathrm{C}\left(\mathrm{CH}_{3}\right)_{2}\left(\mathrm{C}_{6} \mathrm{H}_{5}\right)\right)$ ppm

${ }^{13} \mathrm{C}\left\{{ }^{1} \mathrm{H}\right\}$ NMR $\left(126 \mathrm{MHz}, \mathrm{C}_{6} \mathrm{D}_{6}\right) \delta 151.1(\mathrm{Ar}-\mathrm{C}), 147.1(\mathrm{Ar}-\mathrm{C}), 132.4(\mathrm{Ar}-\mathrm{C}), 131.9(\mathrm{Ar}-\mathrm{C})$, $128.3(\mathrm{Ar}-C), 126.8(\mathrm{Ar}-C), 126.6(\mathrm{Ar}-C), 125.9(\mathrm{Ar}-C), 125.4(\mathrm{Ar}-C), 124.5(\mathrm{Ar}-C), 105.1$ $\begin{array}{lllll}\left(-\mathrm{CH}_{2} \mathrm{C}\left(\mathrm{CH}_{3}\right)_{2}\left(\mathrm{C}_{6} \mathrm{H}_{5}\right)\right), & 84.2 & \left(-\mathrm{OC}\left(\mathrm{CF}_{3}\right)_{2}\right), & 42.1 & \left(-\mathrm{CH}_{2} \mathrm{C}\left(\mathrm{CH}_{3}\right)_{2}\left(\mathrm{C}_{6} \mathrm{H}_{5}\right)\right),\end{array} 3.1$ $\left(-\mathrm{CH}_{2} \mathrm{C}\left(\mathrm{CH}_{3}\right)_{2}\left(\mathrm{C}_{6} \mathrm{H}_{5}\right)\right), 32.9\left(-\mathrm{CH}_{2} \mathrm{C}\left(\mathrm{CH}_{3}\right)_{2}\left(\mathrm{C}_{6} \mathrm{H}_{5}\right)\right), 20.1\left(\mathrm{Ar}-\mathrm{CH}_{3}\right) \mathrm{ppm}$.

${ }^{19} \mathrm{~F}$ NMR $\left(282 \mathrm{MHz}, \mathrm{C}_{6} \mathrm{D}_{6}\right) \delta-70.0\left(\mathrm{q}, 6 \mathrm{~F},{ }^{4} J_{\mathrm{FF}}=9.9 \mathrm{~Hz}\right),-74.7\left(\mathrm{q}, 6 \mathrm{~F},{ }^{4} J_{\mathrm{FF}}=9.8 \mathrm{~Hz}\right) \mathrm{ppm}$. 
Anal. Calcd. for $\mathrm{C}_{40} \mathrm{H}_{38} \mathrm{~F}_{12} \mathrm{NO}_{2} \mathrm{Ta}$ (973.68 g/mol): C, 49.34\%; H, 3.93; N, 1.44; Found: $\mathrm{C}$, $46.67 \% ; \mathrm{H}, 3.82 \% ; \mathrm{N}, 1.44 \%$. 
NMR Characterization of $\left[\mathrm{CF}_{3}-\mathrm{ONO}\right] \mathrm{Ta}\left(\mathrm{CH}_{2} \mathrm{C}\left(\mathrm{CH}_{3}\right)_{2}\left(\mathrm{C}_{6} \mathrm{H}_{5}\right)\right)(9)$

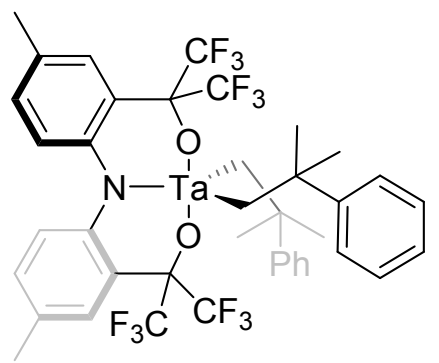

9

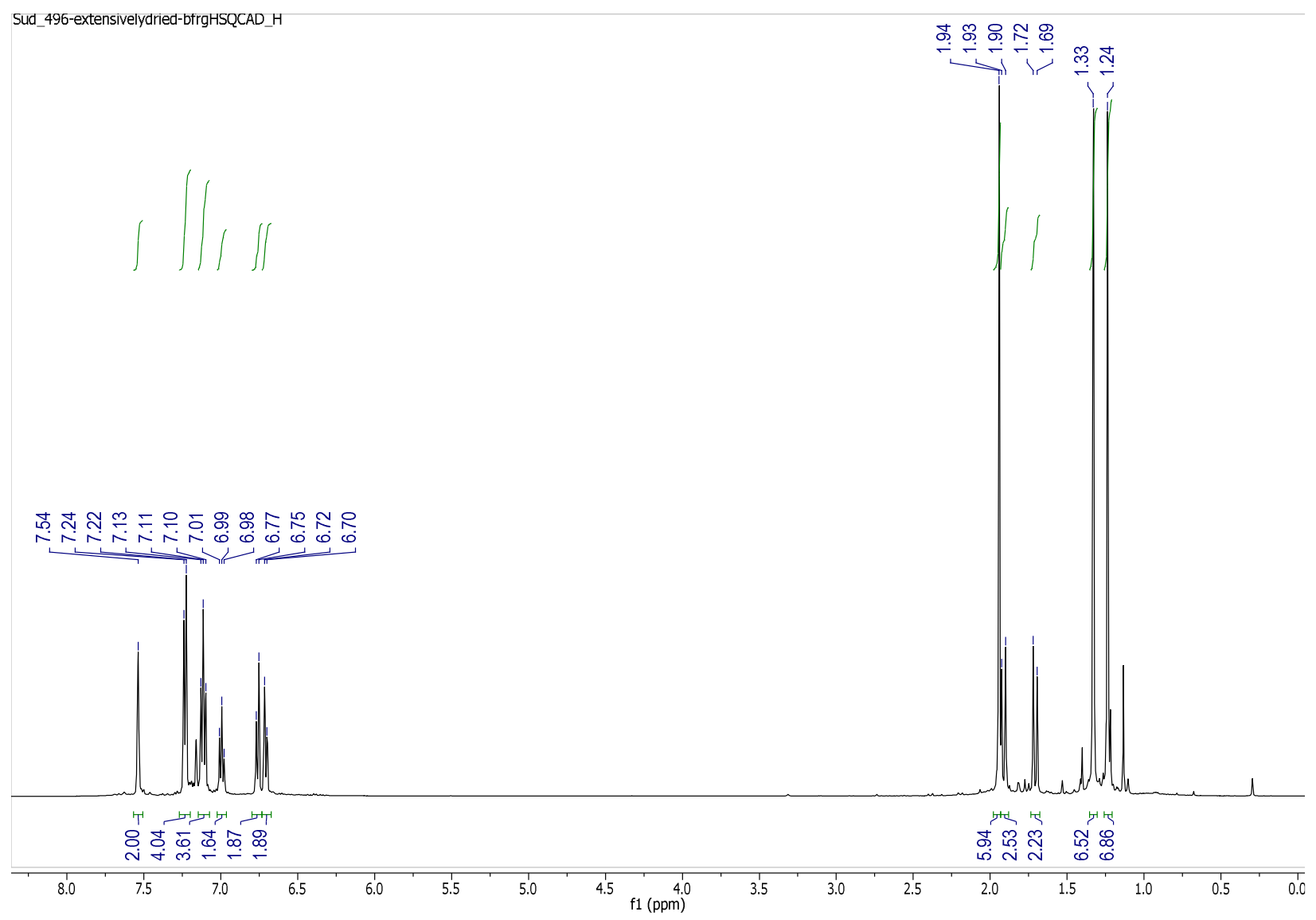

Figure S79. ${ }^{1} \mathrm{H}$ NMR spectrum of $9\left(\mathrm{C}_{6} \mathrm{D}_{6}, 500 \mathrm{MHz}\right)$ 

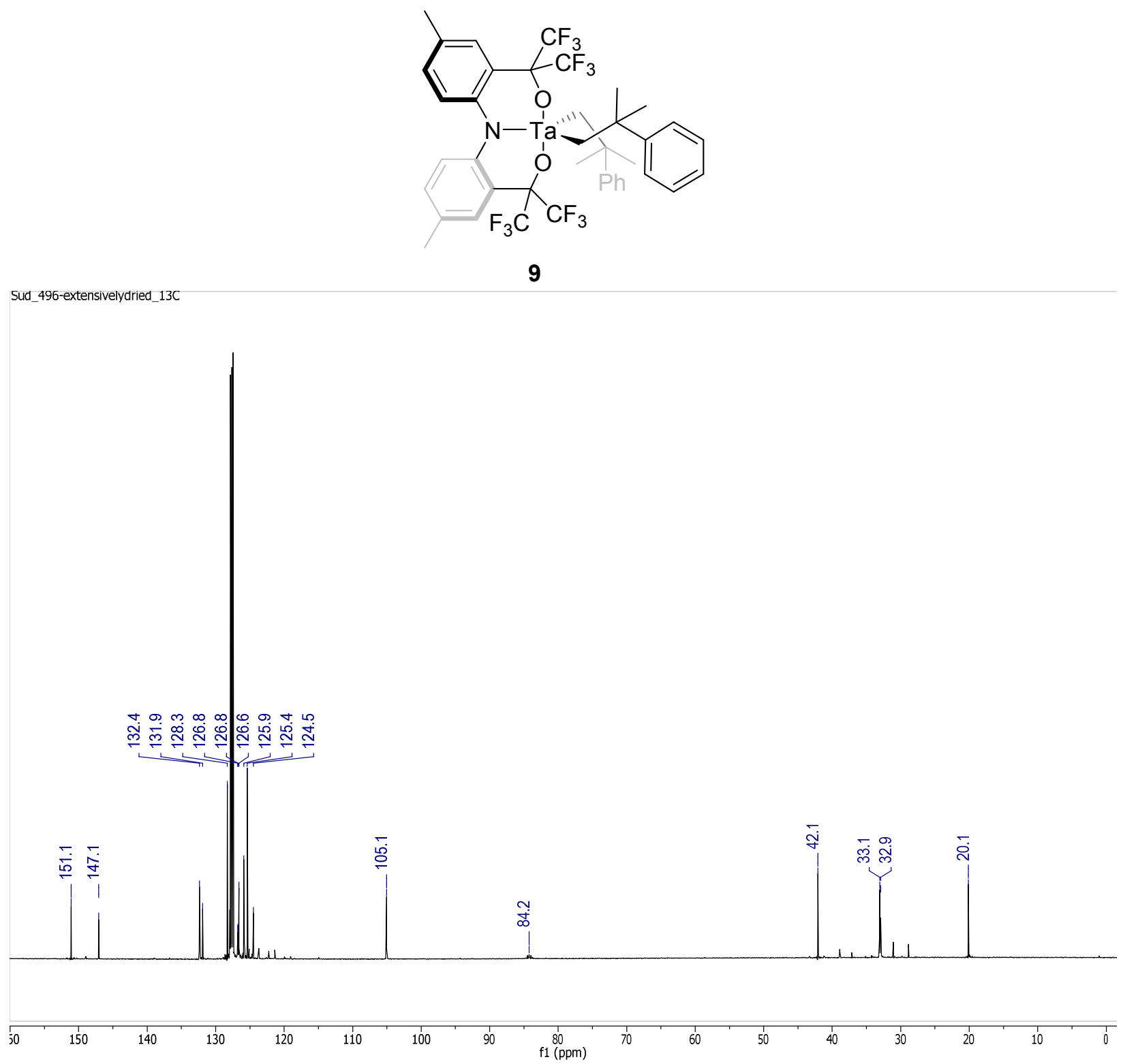

Figure S80. ${ }^{13} \mathrm{C}\left\{{ }^{1} \mathrm{H}\right\}$ NMR spectrum of $9\left(\mathrm{C}_{6} \mathrm{D}_{6}, 126 \mathrm{MHz}\right)$ 


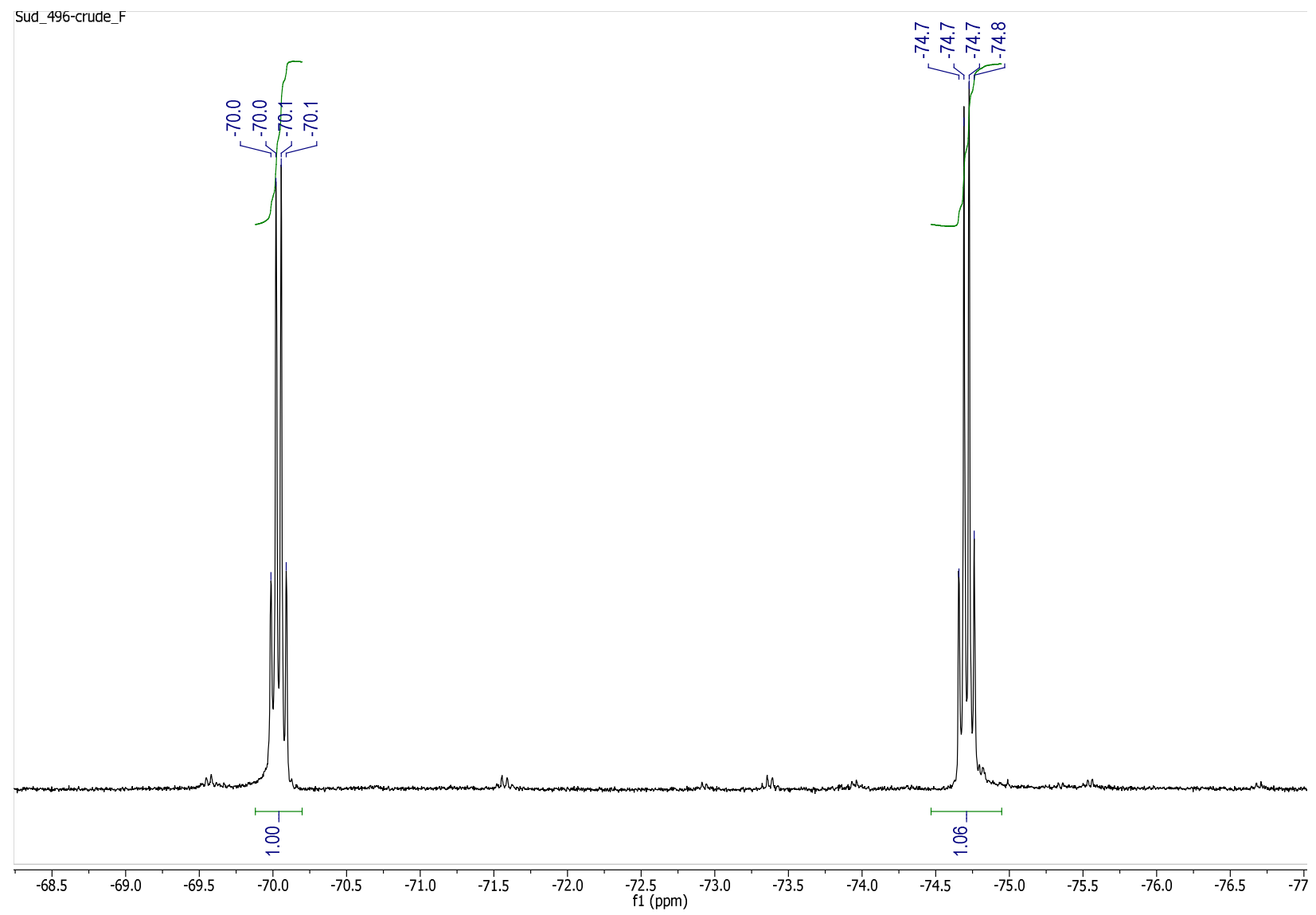

Figure S81. ${ }^{19} \mathrm{~F}$ NMR spectrum of $9\left(\mathrm{C}_{6} \mathrm{D}_{6}, 282 \mathrm{MHz}\right)$ 


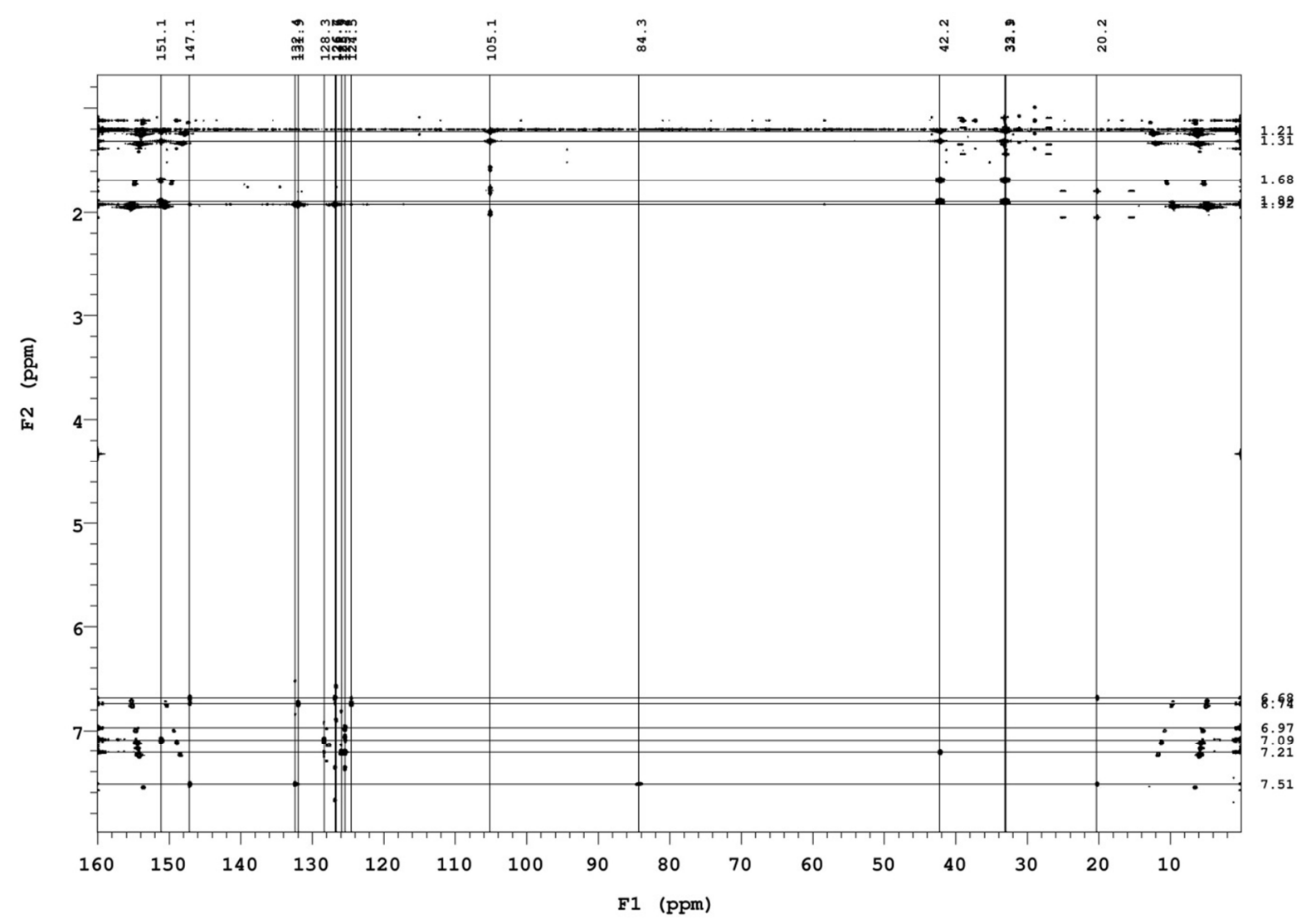

Figure S82. ${ }^{1} \mathrm{H}^{13}{ }^{13} \mathrm{gHMBC}$ spectrum of $9\left(\mathrm{C}_{6} \mathrm{D}_{6}, 500 \mathrm{MHz}\right)$ 


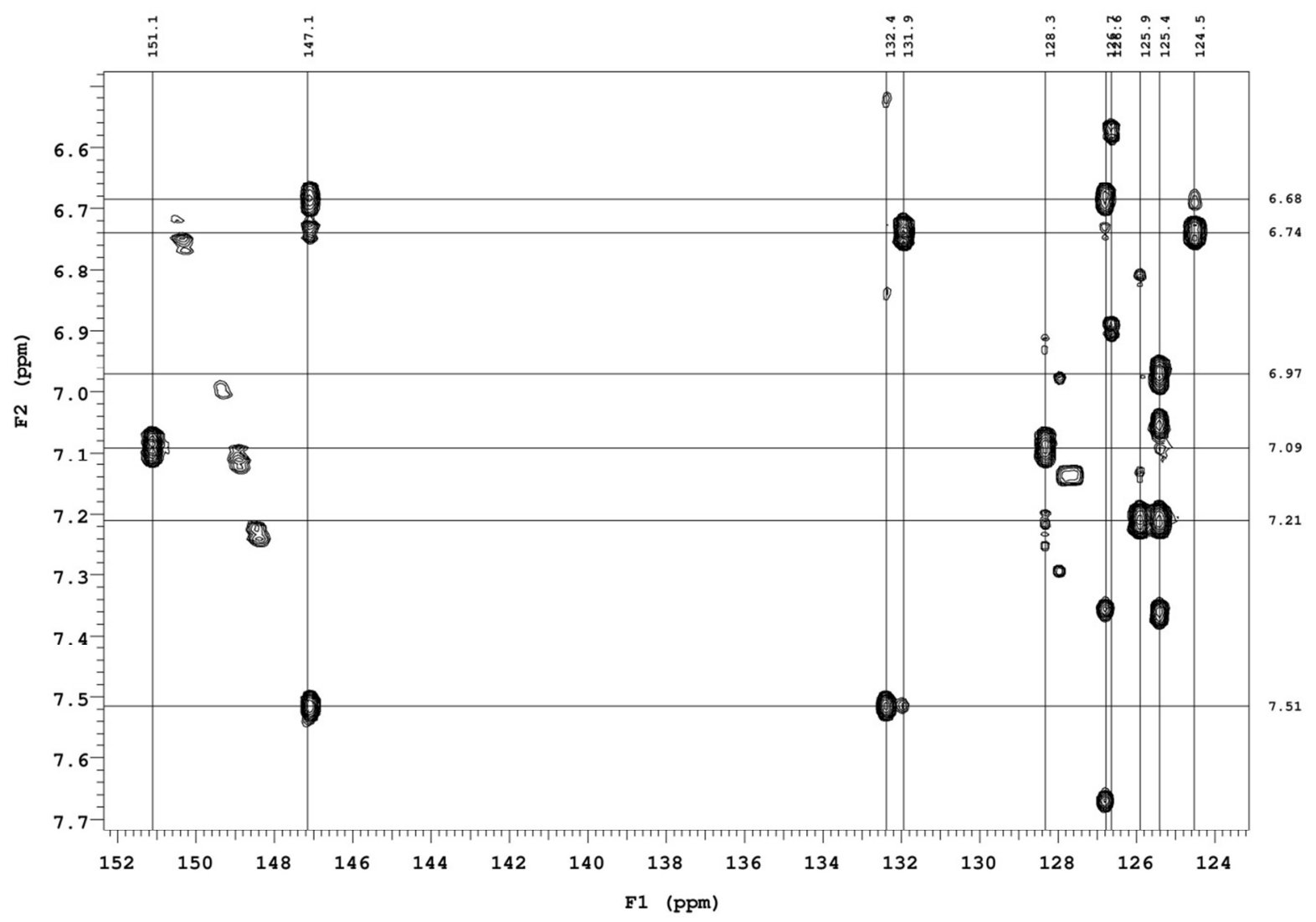

Figure S83. ${ }^{1} \mathrm{H}-{ }^{13} \mathrm{C} g \mathrm{HMBC}$ spectrum of $9-$ Expansion $\left(\mathrm{C}_{6} \mathrm{D}_{6}, 500 \mathrm{MHz}\right)$ 


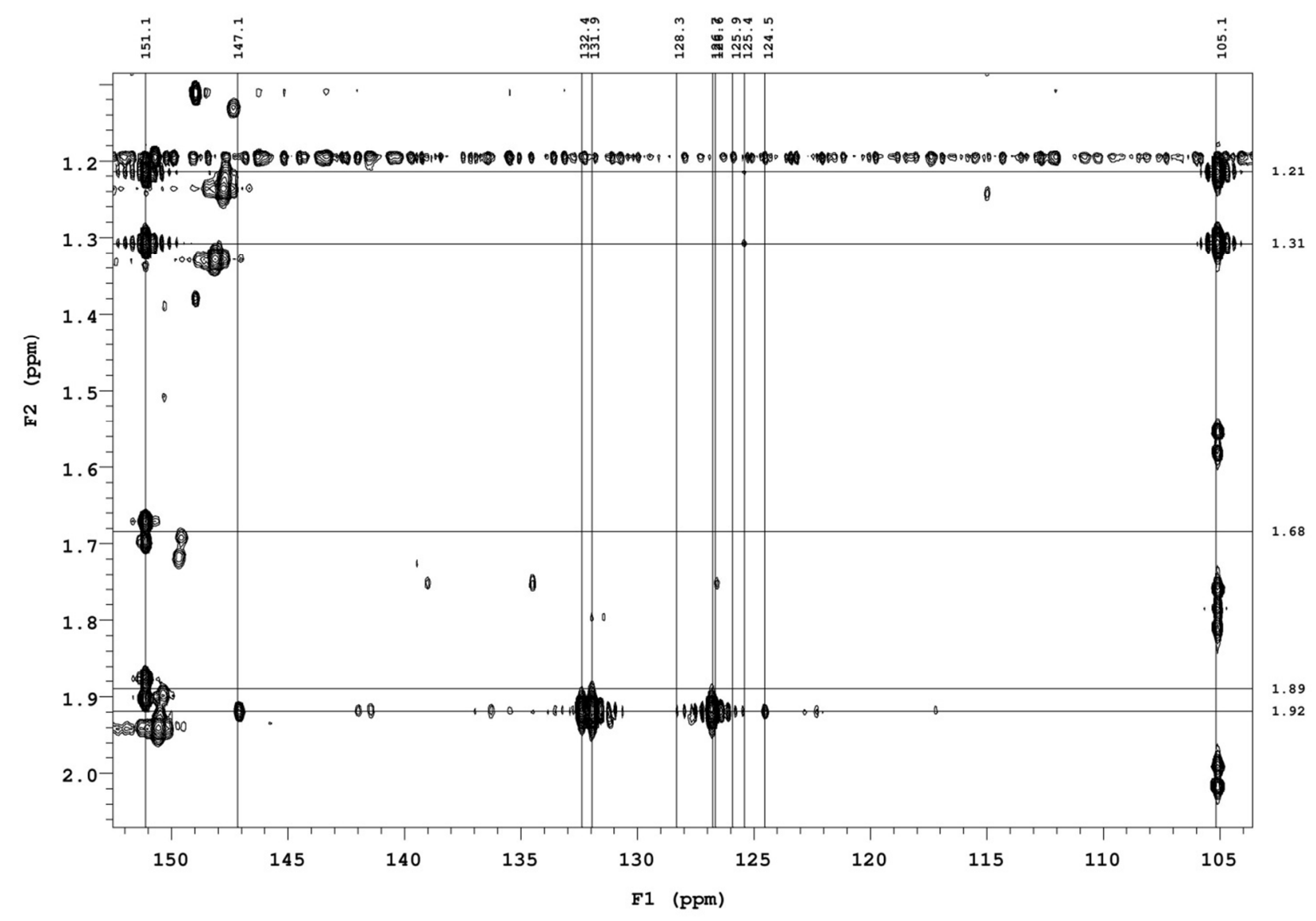

Figure S84. ${ }^{1} \mathrm{H}-{ }^{13} \mathrm{C} g \mathrm{HMBC}$ spectrum of 9 - Expansion $\left(\mathrm{C}_{6} \mathrm{D}_{6}, 500 \mathrm{MHz}\right)$ 


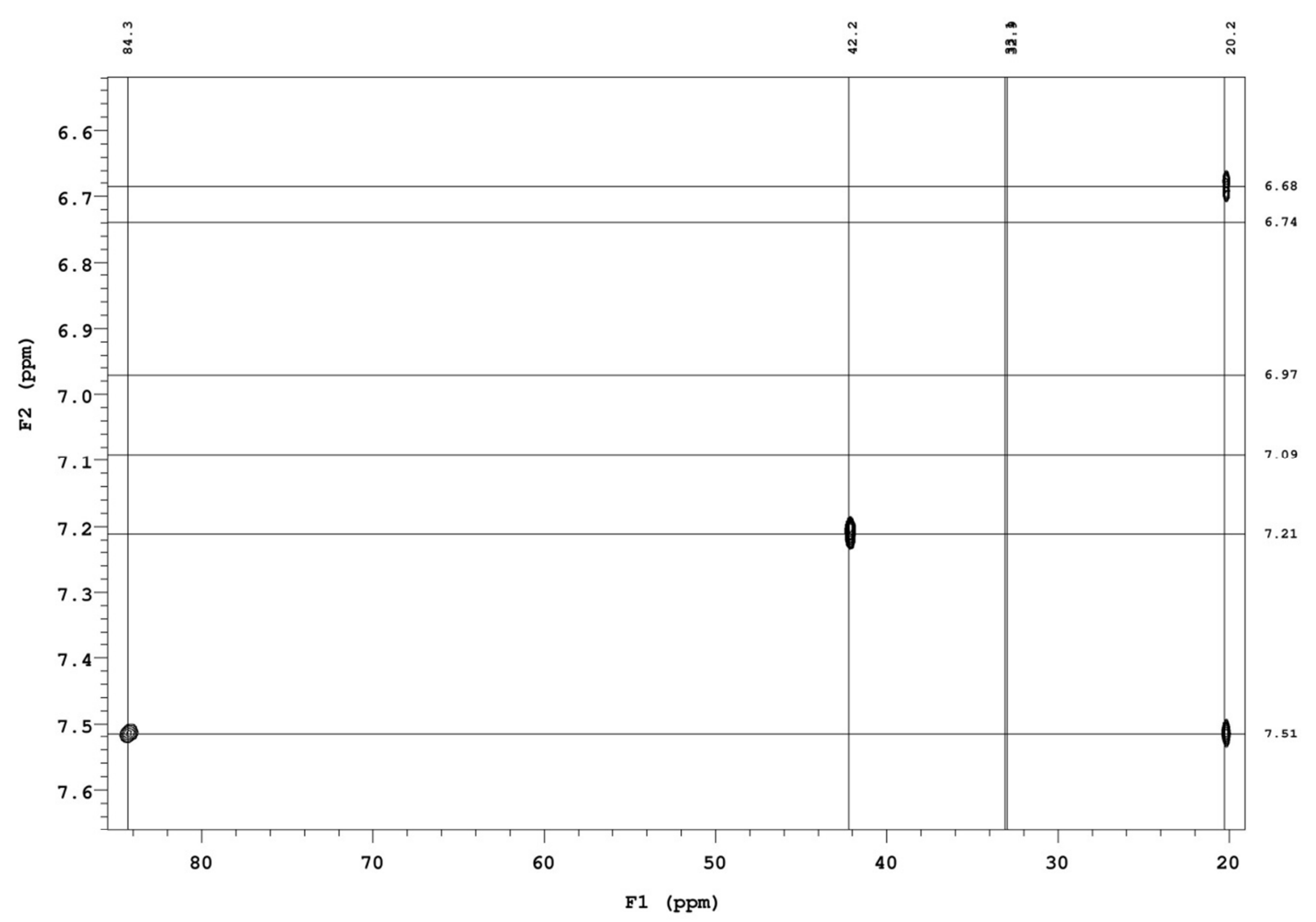

Figure S85 ${ }^{1} \mathrm{H}-{ }^{13} \mathrm{C} g \mathrm{HMBC}$ spectrum of 9 - Expansion $\left(\mathrm{C}_{6} \mathrm{D}_{6}, 500 \mathrm{MHz}\right)$ 


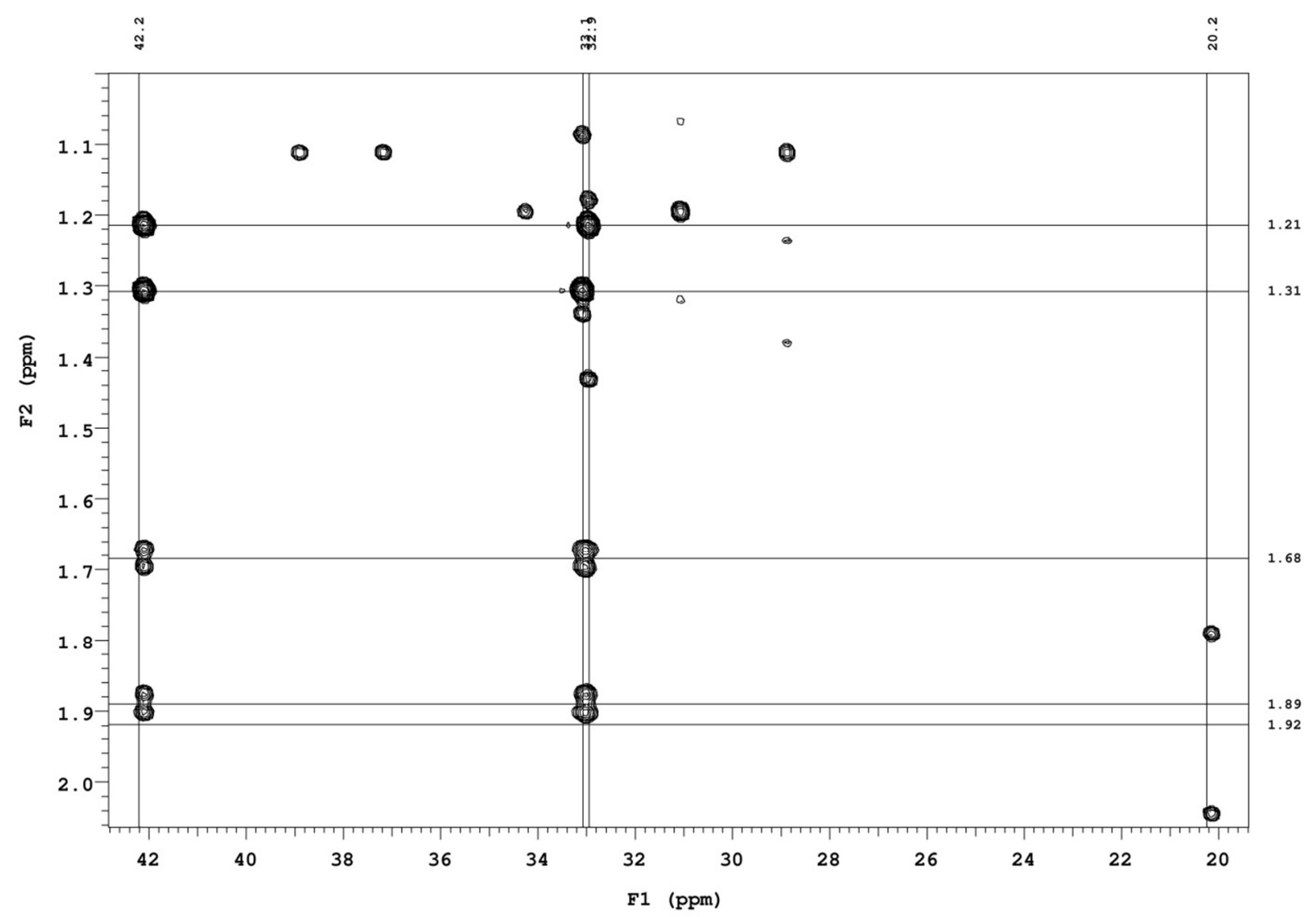

Figure S86. ${ }^{1} \mathrm{H}_{-}{ }^{13} \mathrm{C} g \mathrm{HMBC}$ spectrum of 9 - Expansion $\left(\mathrm{C}_{6} \mathrm{D}_{6}, 500 \mathrm{MHz}\right)$

Polymerization of norbornene by $\left[\mathrm{CF}_{3}-\mathrm{ONO}\right] \mathrm{Nb}\left(\mathrm{CH}_{2} \mathrm{R}\right)_{2}\left(\right.$ where $\mathrm{R}=\mathrm{C}_{6} \mathrm{H}_{5}(5), \mathrm{Si}\left(\mathrm{CH}_{3}\right)_{3}$ (6), and $\left.\left.-\mathrm{C}\left(\mathrm{CH}_{3}\right)_{2}\left(\mathrm{C}_{6} \mathrm{H}_{5}\right)\right)(7)\right)$

A detailed procedure for the polymerization trials is provided for 7 . The same procedure also applies for complexes $\mathbf{5}$ and $\mathbf{6}$. The results are summarized in Table S2.

\section{Polymerization of norbornene by $\left[\mathrm{CF}_{3}-\mathrm{ONO}\right] \mathrm{Nb}\left(\mathrm{CH}_{2} \mathrm{C}\left(\mathrm{CH}_{3}\right)_{2}\left(\mathrm{C}_{6} \mathrm{H}_{5}\right)\right)(7)$}

In a glove box, norbornene $\left(0.032 \mathrm{~g}, 3.4 \times 10^{-4} \mathrm{~mol}, 100\right.$ equiv $)$ was dissolved in $1 \mathrm{~mL}$ toluene. 3 $\mathrm{mL}$ of a $1 \mathrm{mg} / \mathrm{mL}$ solution of $7\left(0.003 \mathrm{~g}, 3.4 \times 10^{-6}\right.$ mol, 1 equiv $)$ dissolved in toluene was added to the norbornene solution and mixed well with a pipette. The mixture was then transferred to a 
round bottom flask, sealed, and taken outside the glove box. The reaction was stirred for $15 \mathrm{~h}$ at $60{ }^{\circ} \mathrm{C}$. After this period, the reaction was added dropwise into stirring methanol to precipitate polynorbornene, which was isolated by gravity filtration and vacuum dried. (Yield: $0.017 \mathrm{~g}$, $53 \%) .{ }^{1} \mathrm{H}$ and ${ }^{13} \mathrm{C}$ NMR spectral assignments were consistent with literature reports. ${ }^{4}$

Comparable polymerization trials were carried out in sealable NMR tubes. In a typical procedure, complex $7\left(0.003 \mathrm{~g}, 3.4 \times 10^{-6} \mathrm{~mol}\right)$ dissolved in approximately $1.5 \mathrm{~mL} \mathrm{C}_{6} \mathrm{D}_{6}$, was added to solid norbornene $\left(0.032 \mathrm{~g}, 3.4 \times 10^{-4} \mathrm{~mol}\right)$ and mixed well. The reaction mixture was then transferred to a sealable NMR tube and a spectrum was acquired to denote onset of reaction. The NMR tube was placed in a pre-heated oil bath $\left(60^{\circ} \mathrm{C}\right)$ for approximately $15 \mathrm{~h}$, after which another spectrum was acquired. The contents of the NMR tube were carefully added to stirring methanol to precipitate polynorbornene. The polymer was isolated by filtration and vacuum dried. (Yield: $0.020 \mathrm{~g}, 62 \%$ )

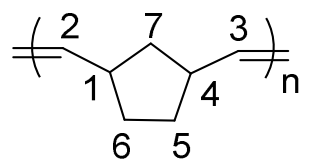

Table S2. Polymerization of norbornene by precatalysts 5-7 with different monomer/catalyst ratios

\begin{tabular}{|c|c|c|c|c|}
\hline Entry & Precatalyst & {$[\text { Monomer/Catalyst }]_{0}$} & Yield (\%) & $\% \operatorname{cis}^{b}$ \\
\hline 1 & 7 & 25:1 (NMR tube) & 60 & 89 \\
\hline 2 & 7 & $50: 1(\text { Sealed RBF })^{\mathrm{a}}$ & 56 & 76 \\
\hline 3 & 7 & 50:1 (NMR tube) & 76 & 85 \\
\hline 4 & 7 & 100:1 (Sealed RBF $)^{\mathrm{a}}$ & 53 & 83 \\
\hline 5 & 7 & 100:1 (NMR tube) & 62 & 85 \\
\hline 6 & 5 & 100:1 (Sealed RBF) ${ }^{\mathrm{a}}$ & 64 & 77 \\
\hline 7 & 6 & 100:1 (Sealed RBF $)^{a}$ & 46 & 86 \\
\hline
\end{tabular}

$a-\mathrm{a}$ toluene solution of the precatalyst was added to norbornene in toluene; [monomer $]_{0}=0.1$ $\mathrm{M}$;

$b$ - determined by ${ }^{1} \mathrm{H}$ NMR spectroscopy $\left(\mathrm{CDCl}_{3}\right)$. 


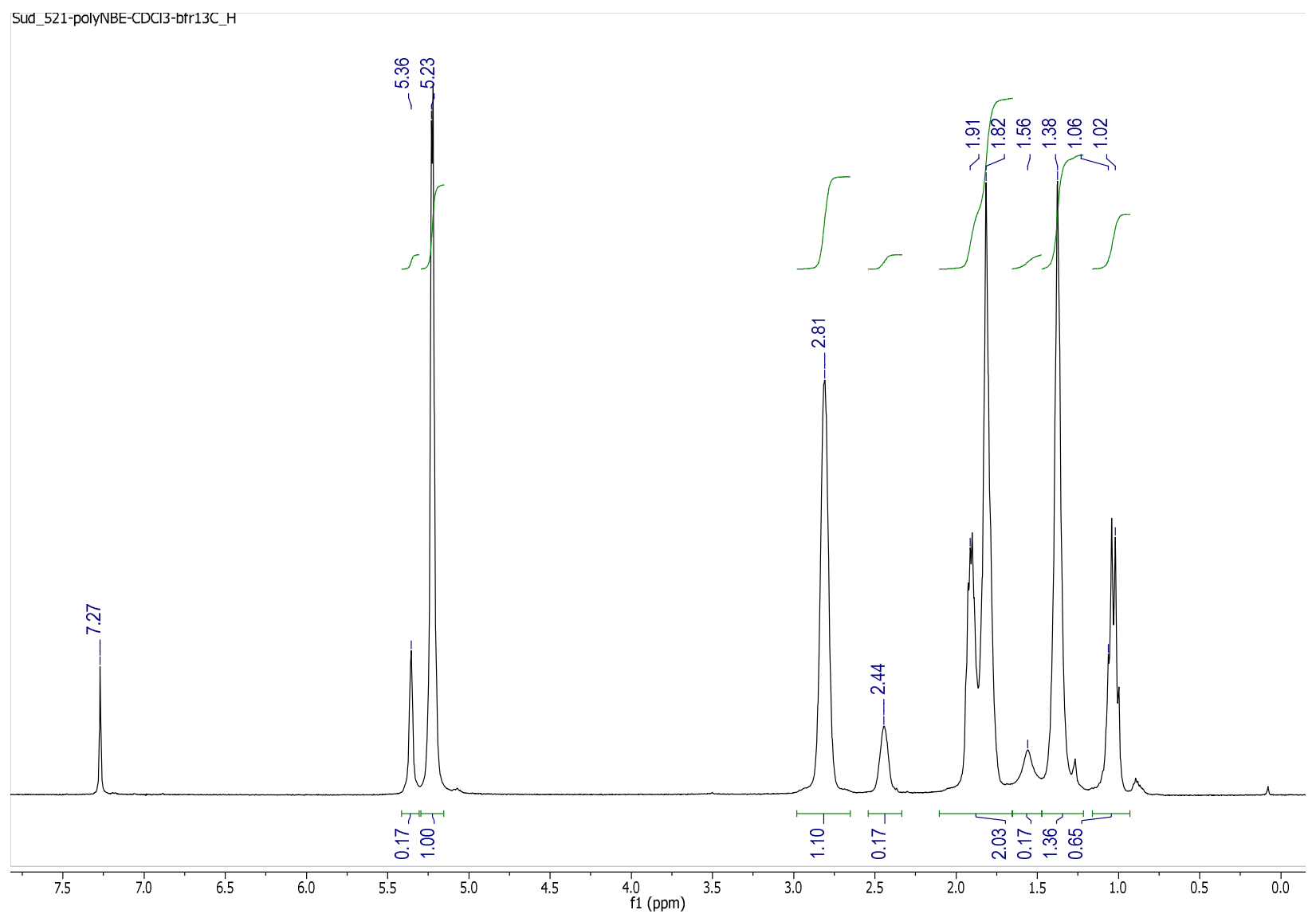

Figure S87. ${ }^{1} \mathrm{H}$ NMR spectrum of $\sim 85 \%$ cis, syndiotactic enriched polynorbornene ${ }^{4}$ generated by $7\left(\mathrm{CDCl}_{3}, 500 \mathrm{MHz}\right)$ 

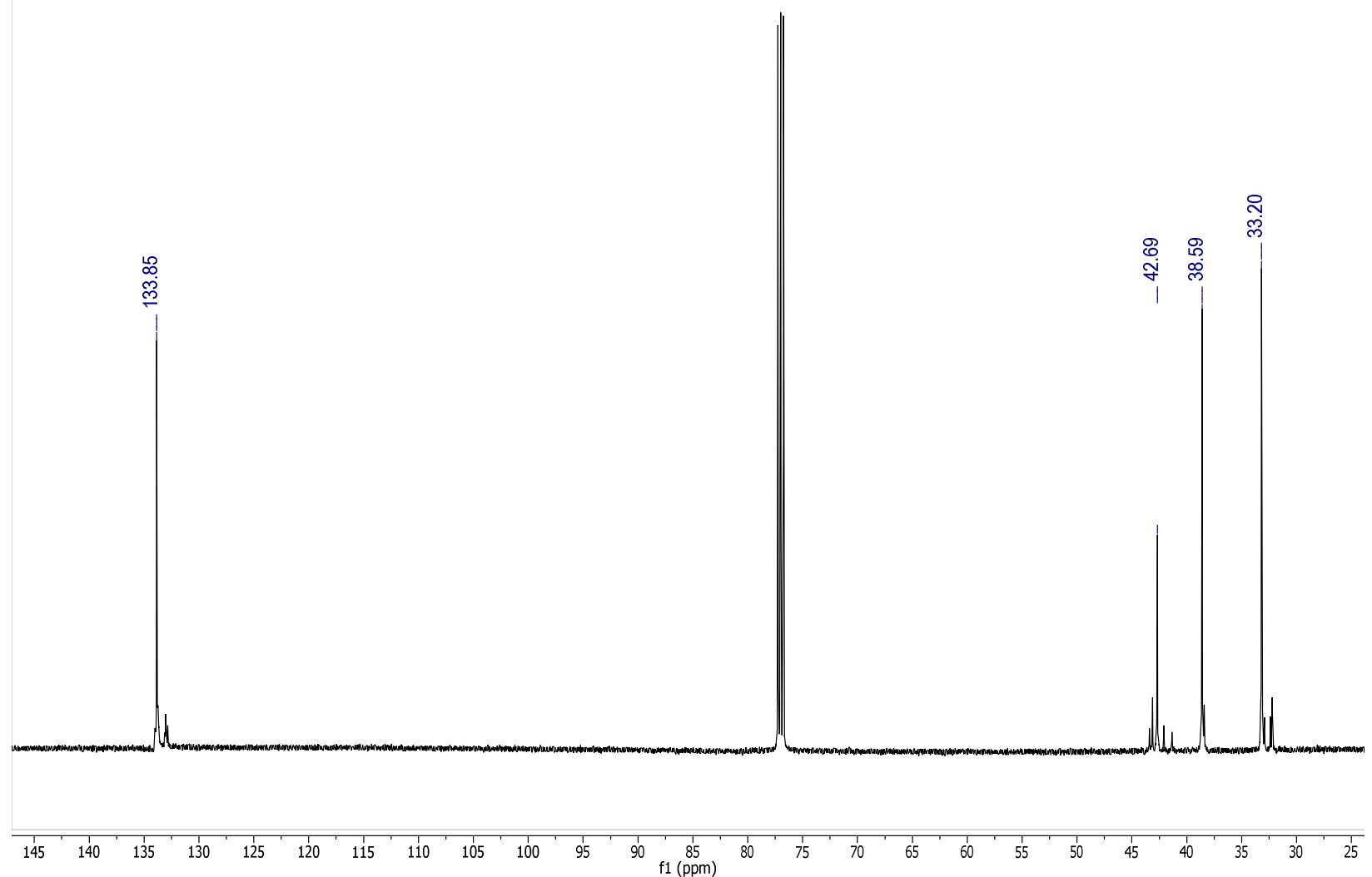

Figure S88. ${ }^{13} \mathrm{C}\left\{{ }^{1} \mathrm{H}\right\}$ NMR spectrum of $\sim 85 \%$ cis, syndiotactic enriched polynorbornene ${ }^{5}$ generated by $7\left(\mathrm{CDCl}_{3}, 125 \mathrm{MHz}\right)$ 


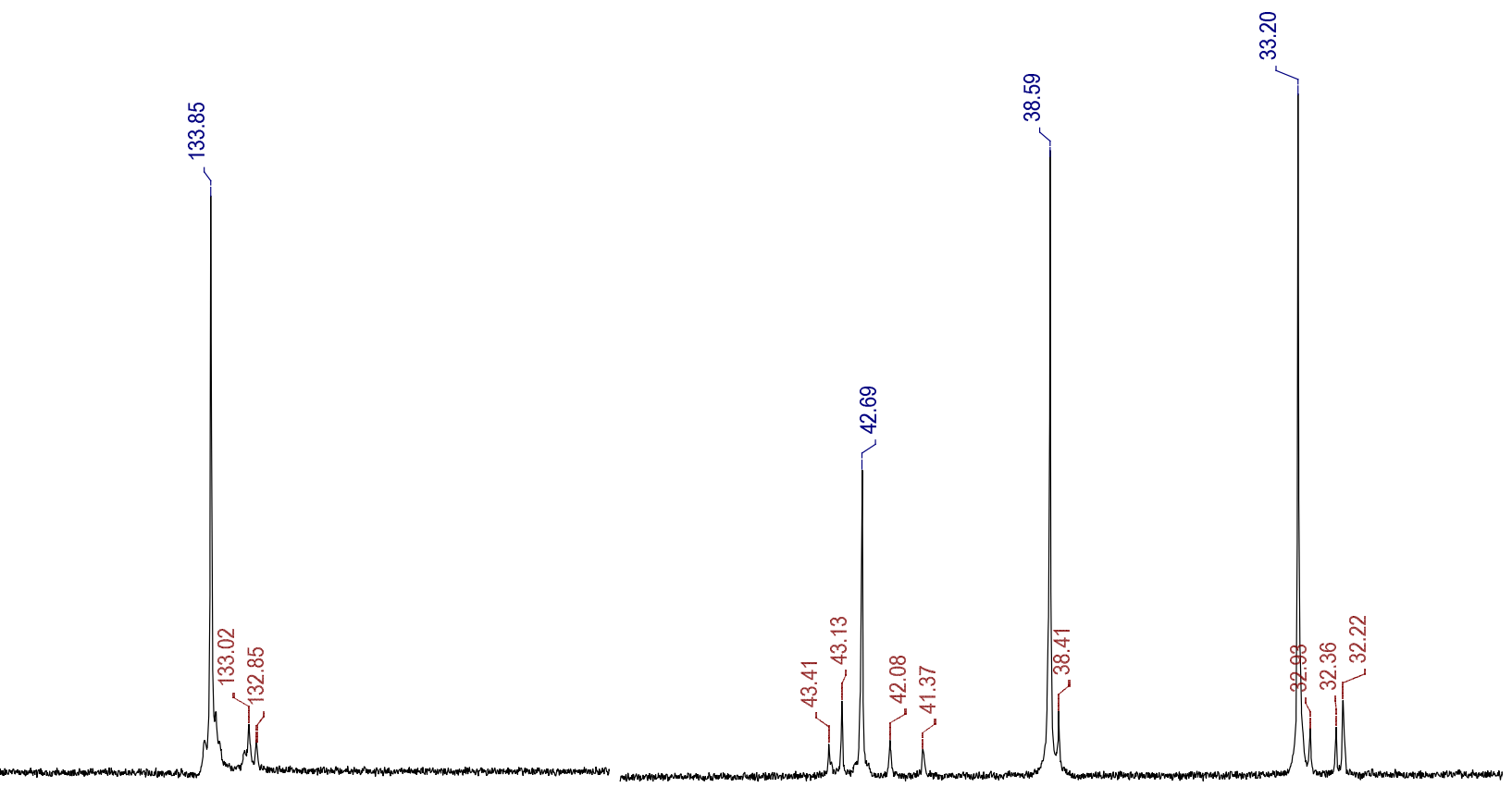

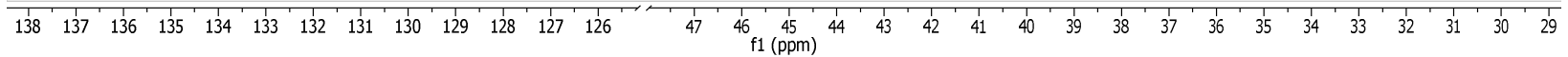

Figure S89. Expansion of the ${ }^{13} \mathrm{C}\left\{{ }^{1} \mathrm{H}\right\}$ NMR spectrum of $\sim 85 \%$ cis, syndiotactic enriched polynorbornene generated by $7\left(\mathrm{CDCl}_{3}, 125 \mathrm{MHz}\right)$ - atactic polynorbornene highlighted in RED 
Sud_532-t=30mins-1000scans_H

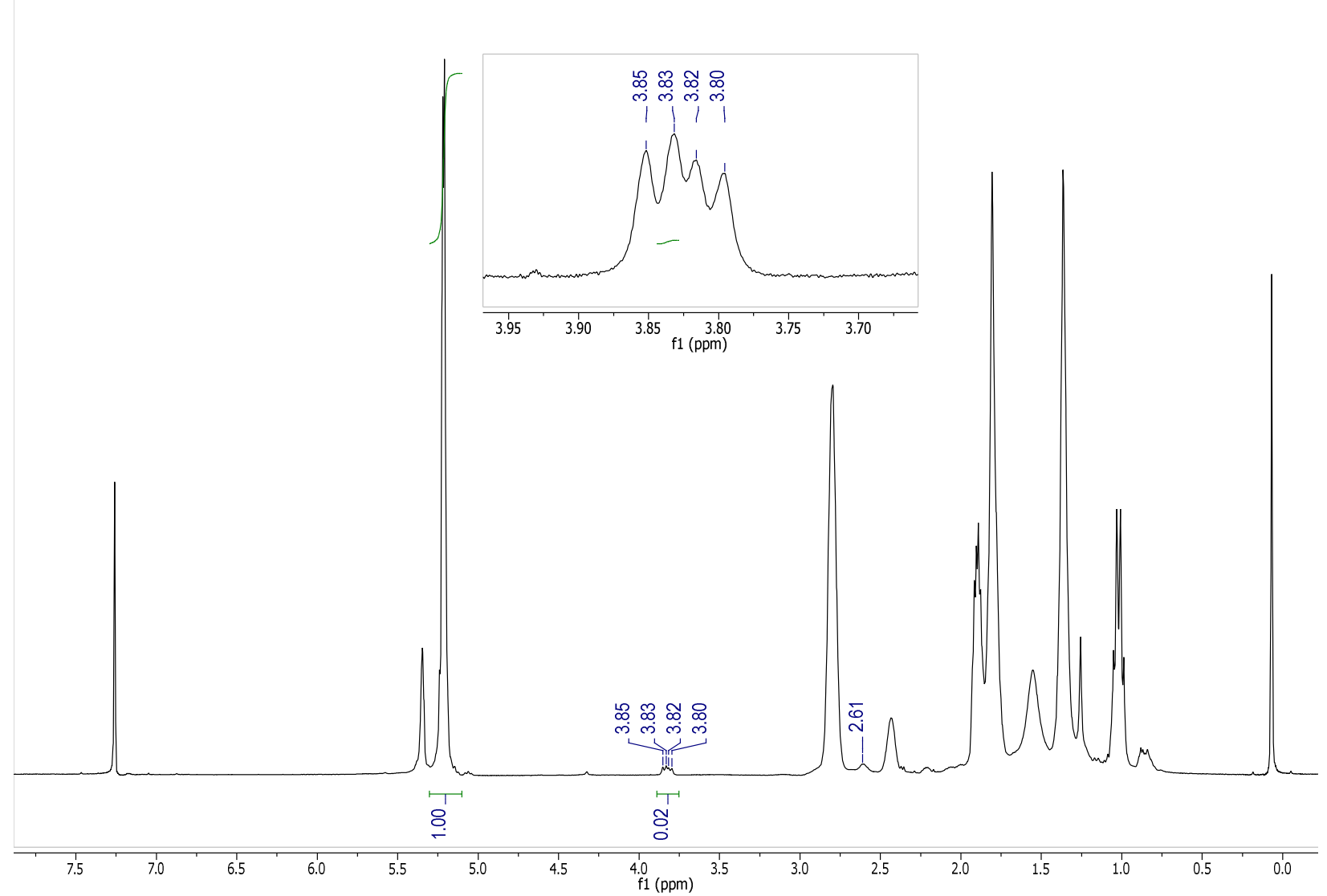

Figure S90. ${ }^{1} \mathrm{H}$ NMR spectrum of partially $(\sim 2 \%)$ brominated $^{6} \sim 85 \%$ cis, syndiotactic enriched polynorbornene generated by $7\left(\mathrm{CDCl}_{3}, 500 \mathrm{MHz}\right)$ 


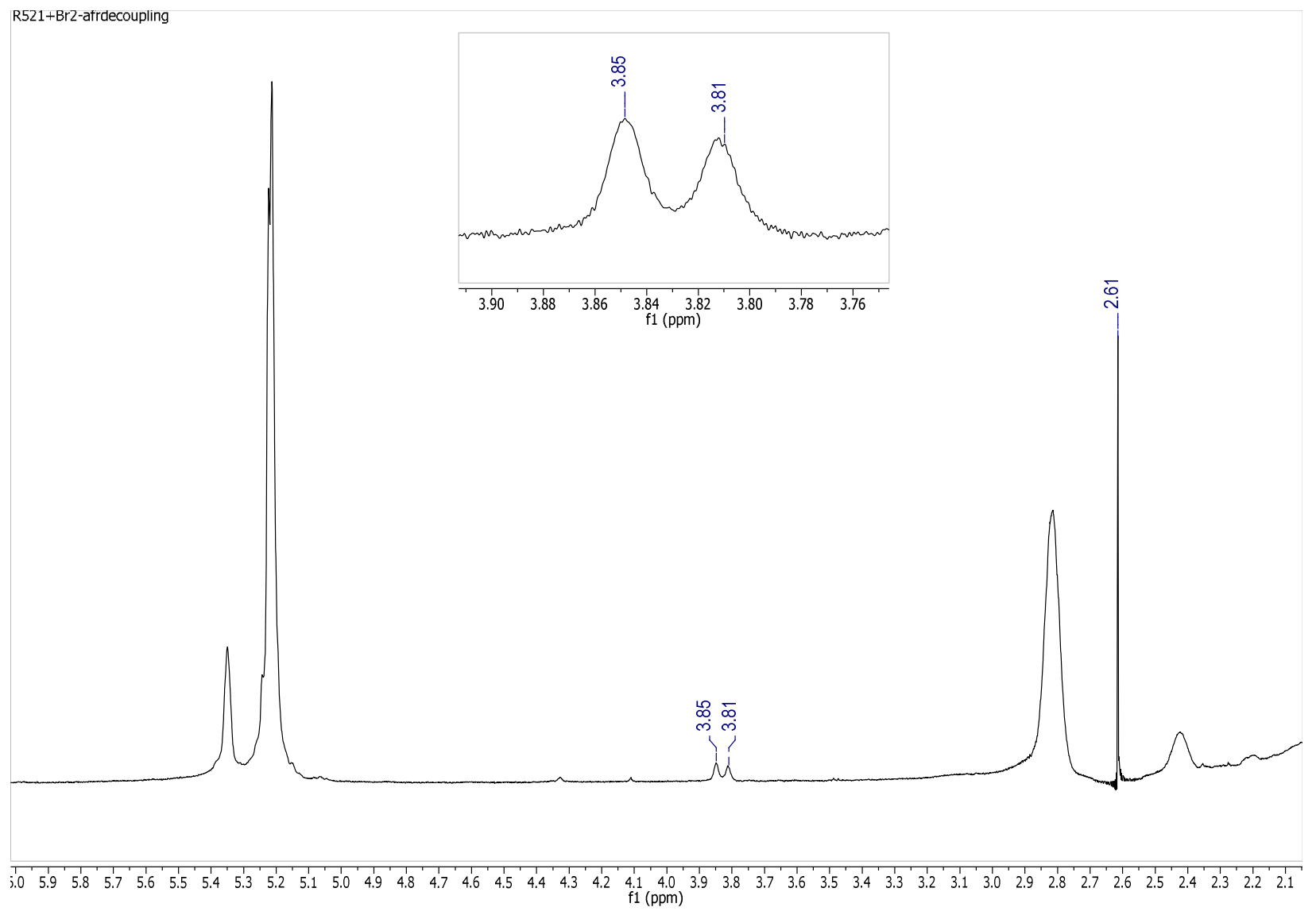

Figure S91. Expansion of the ${ }^{1} \mathrm{H}$ NMR spectrum of partially $(\sim 2 \%)$ brominated $^{6} \sim 85 \%$ cis, syndiotactic enriched polynorbornene generated by 7 , with decoupling of the methine protons at $2.61 \mathrm{ppm}\left(\mathrm{CDCl}_{3}, 500 \mathrm{MHz}\right)$ 


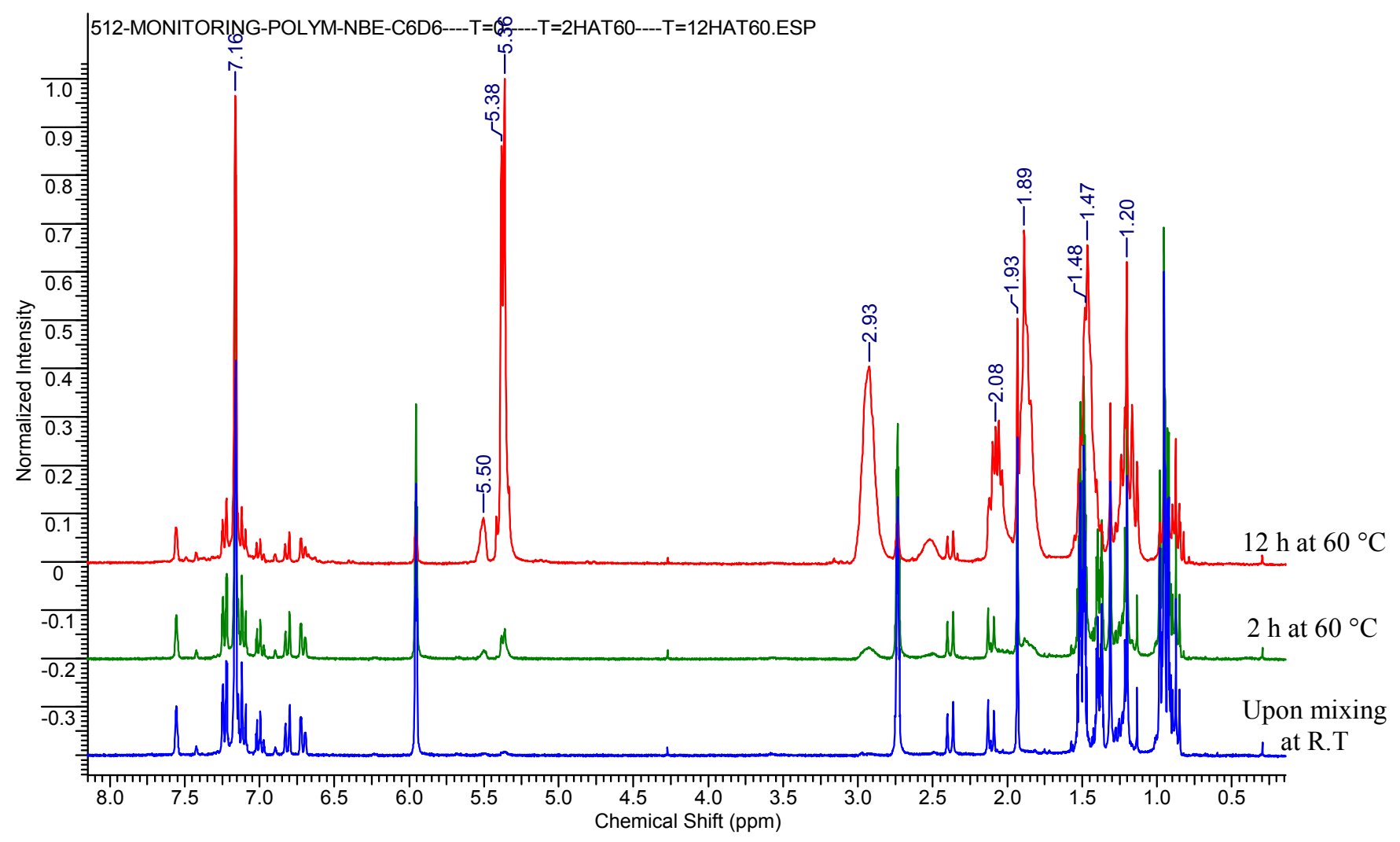

Figure S92. ${ }^{1} \mathrm{H}$ NMR spectra of polynorbornene generated by 7 , in a sealable NMR tube $\left(\mathrm{C}_{6} \mathrm{D}_{6}\right.$, $500 \mathrm{MHz})-$ Presence of resonances pertaining to unreacted 7 indicate poor initiation $\left(\mathrm{k}_{\mathrm{p}}>\mathrm{k}_{\mathrm{i}}\right)$ 


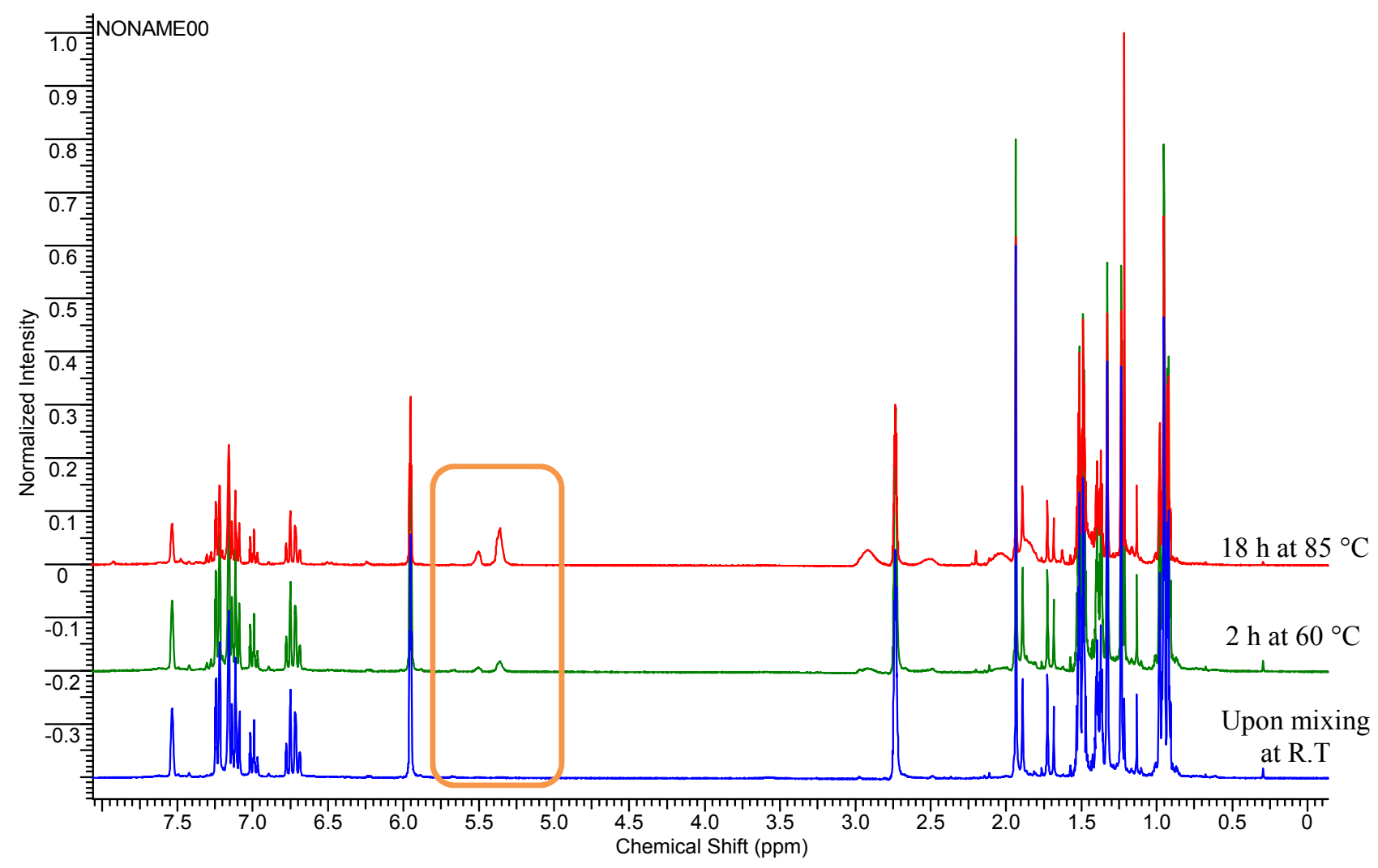

Figure S93. ${ }^{1} \mathrm{H}$ NMR spectra of polynorbornene generated by 9 , in a sealable NMR tube $\left(\mathrm{C}_{6} \mathrm{D}_{6}\right.$, $300 \mathrm{MHz})$

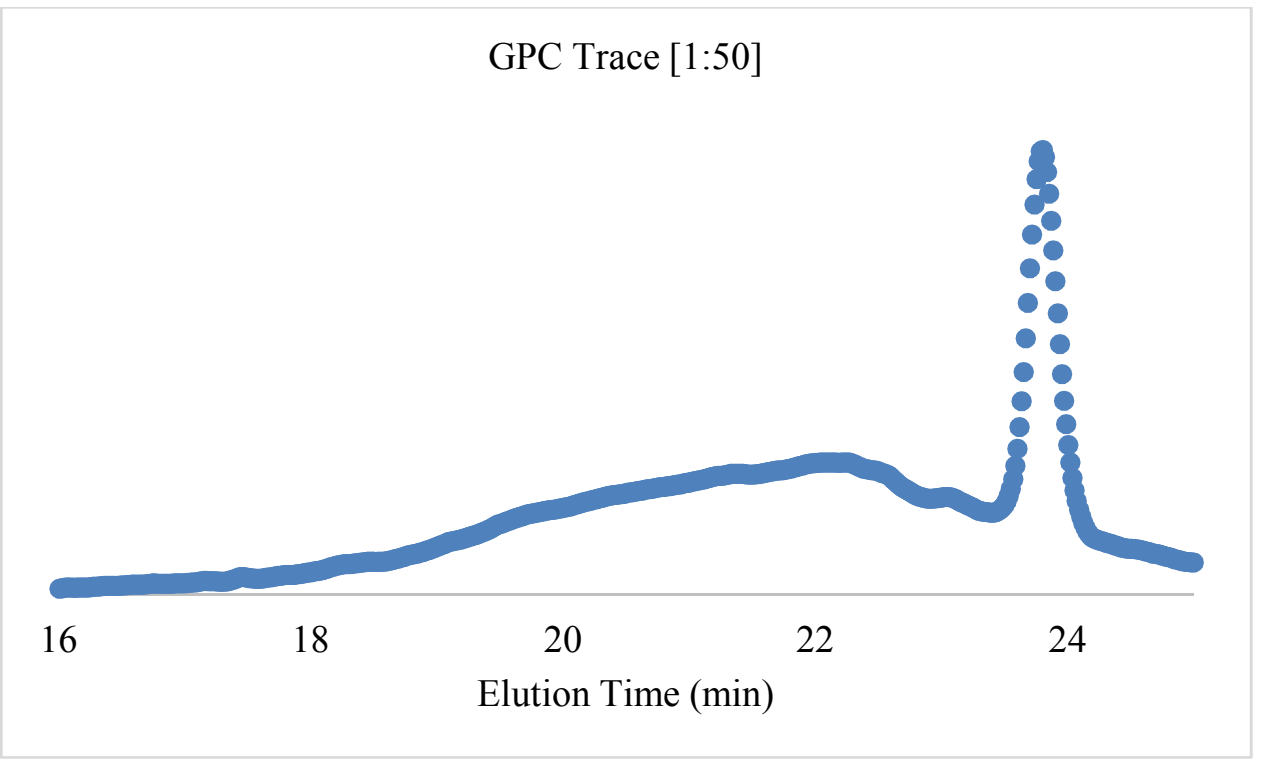

Figure S94. GPC refractive index trace of Entry 2 (Table S2) with a catalyst:monomer ratio of 1:50 in THF. 


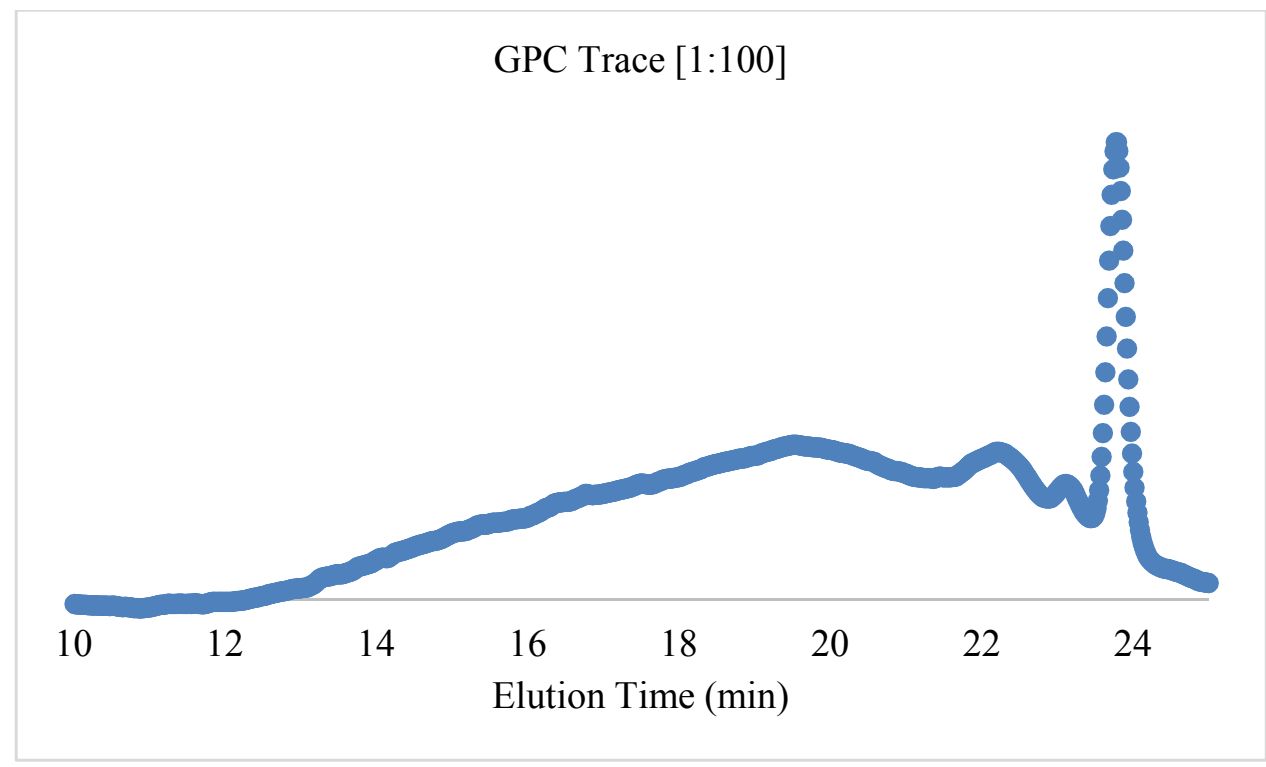

Figure S95. GPC refractive index trace of Entry 4 (Table S2) with a catalyst:monomer ratio of $1: 100$ in THF. 


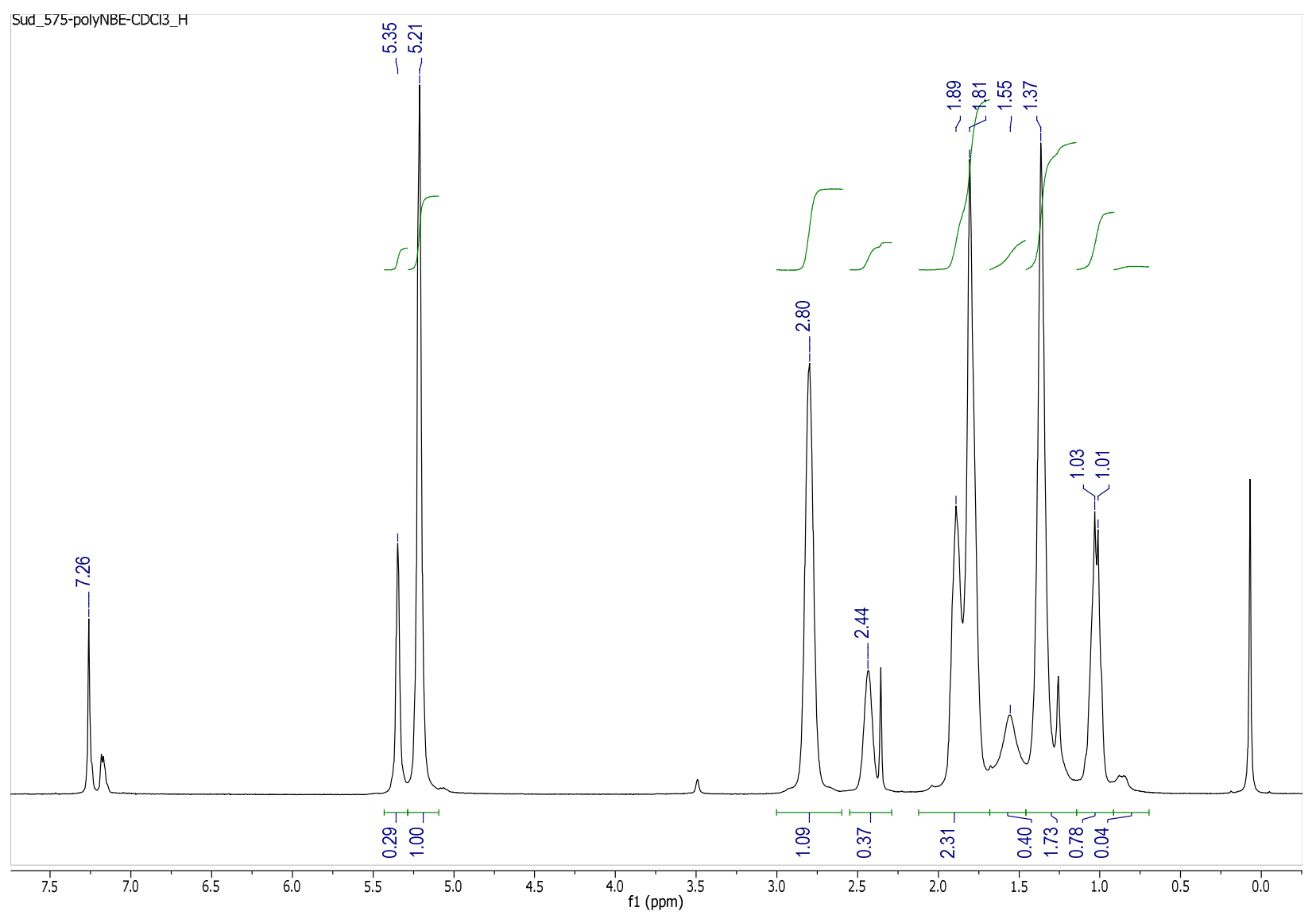

Figure S96. ${ }^{1} \mathrm{H}$ NMR spectrum of $\sim 77 \%$ cis, syndiotactic enriched polynorbornene generated by $5\left(\mathrm{CDCl}_{3}, 500 \mathrm{MHz}\right)$ 


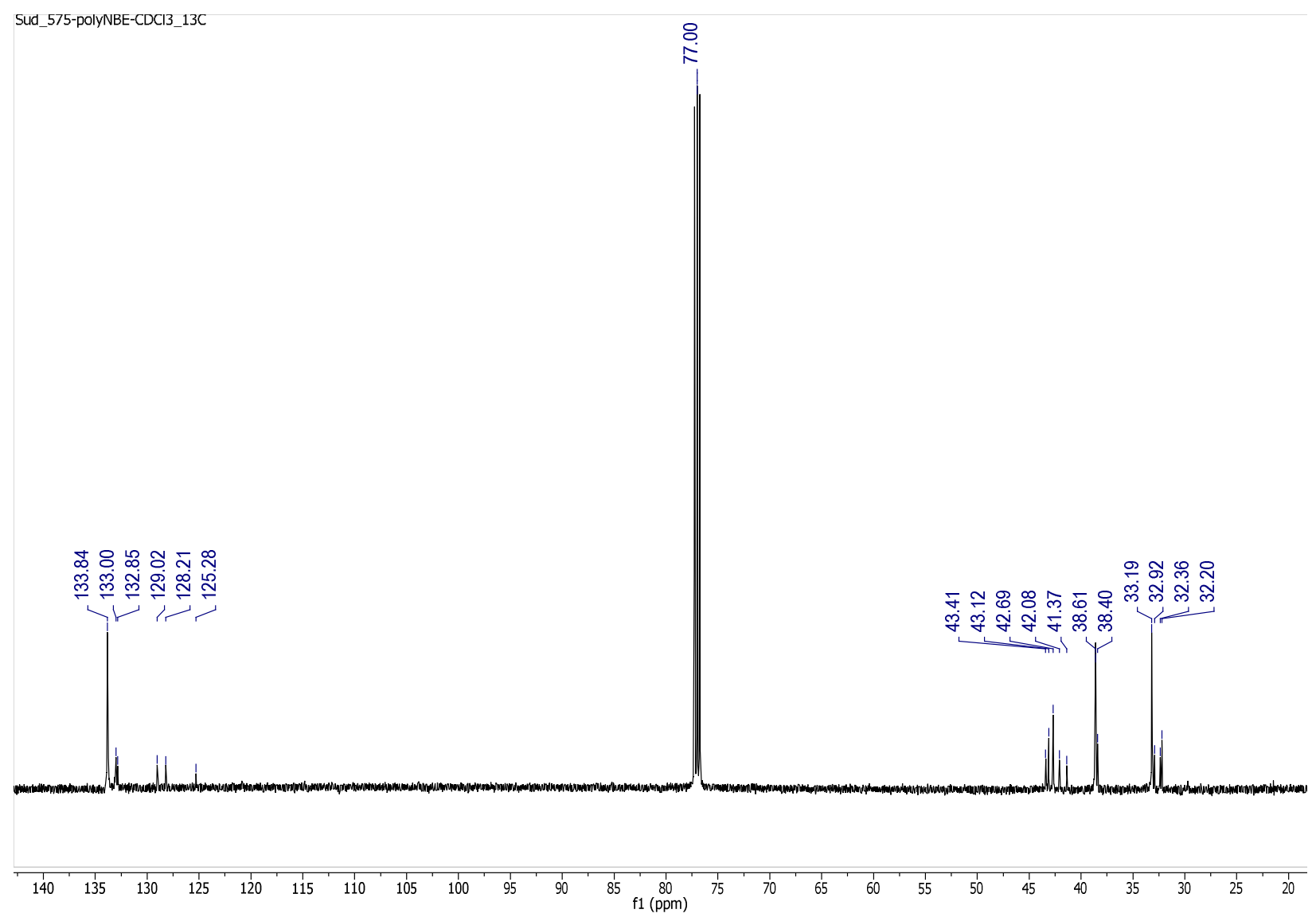

Figure S97. ${ }^{13} \mathrm{C}\left\{{ }^{1} \mathrm{H}\right\}$ NMR spectrum of $\sim 77 \%$ cis, syndiotactic enriched polynorbornene generated by $5\left(\mathrm{CDCl}_{3}, 125 \mathrm{MHz}\right)$ 


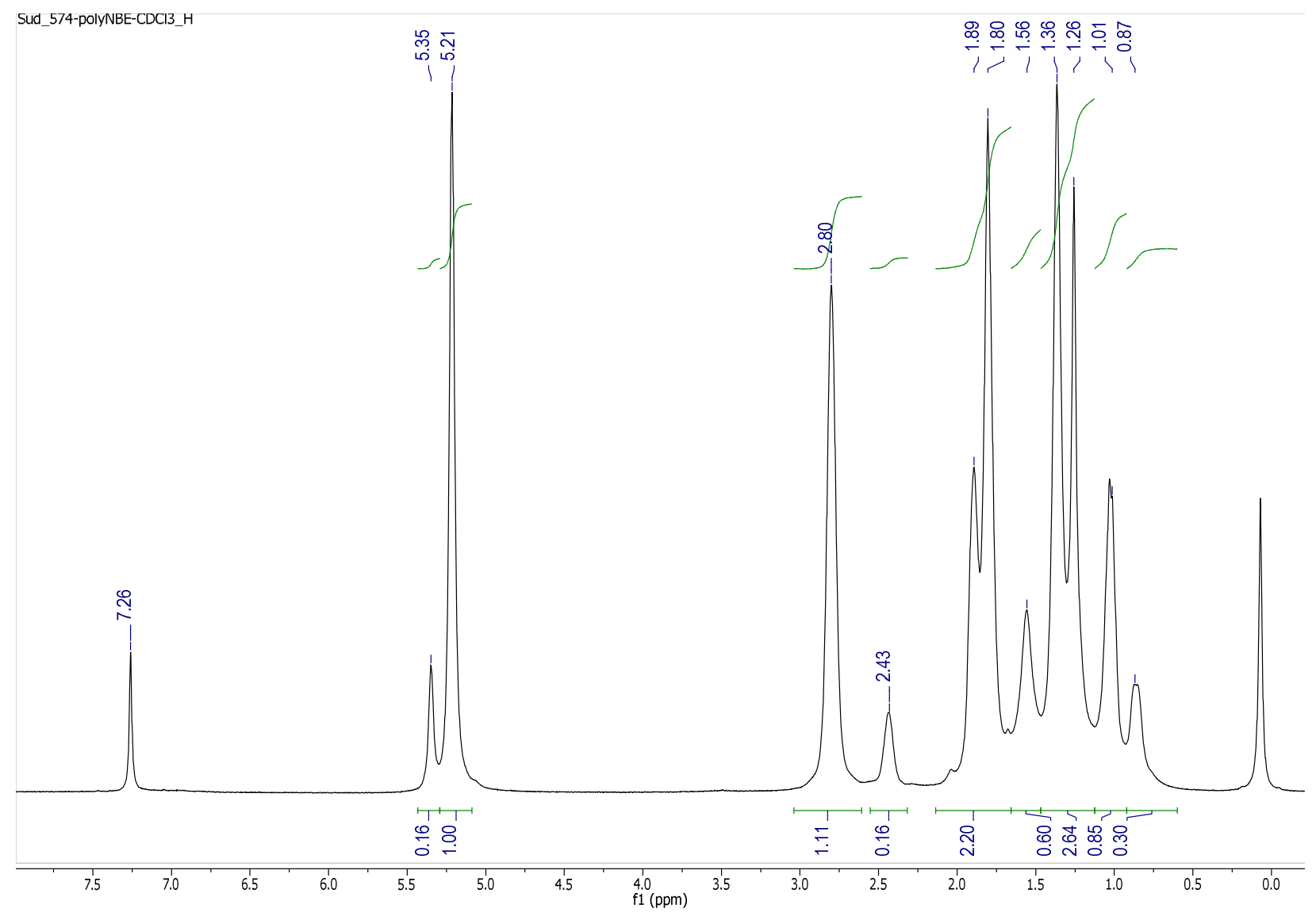

Figure S98. ${ }^{1} \mathrm{H}$ NMR spectrum of $\sim 86 \%$ cis, syndiotactic enriched polynorbornene generated by $6\left(\mathrm{CDCl}_{3}, 500 \mathrm{MHz}\right)$ 


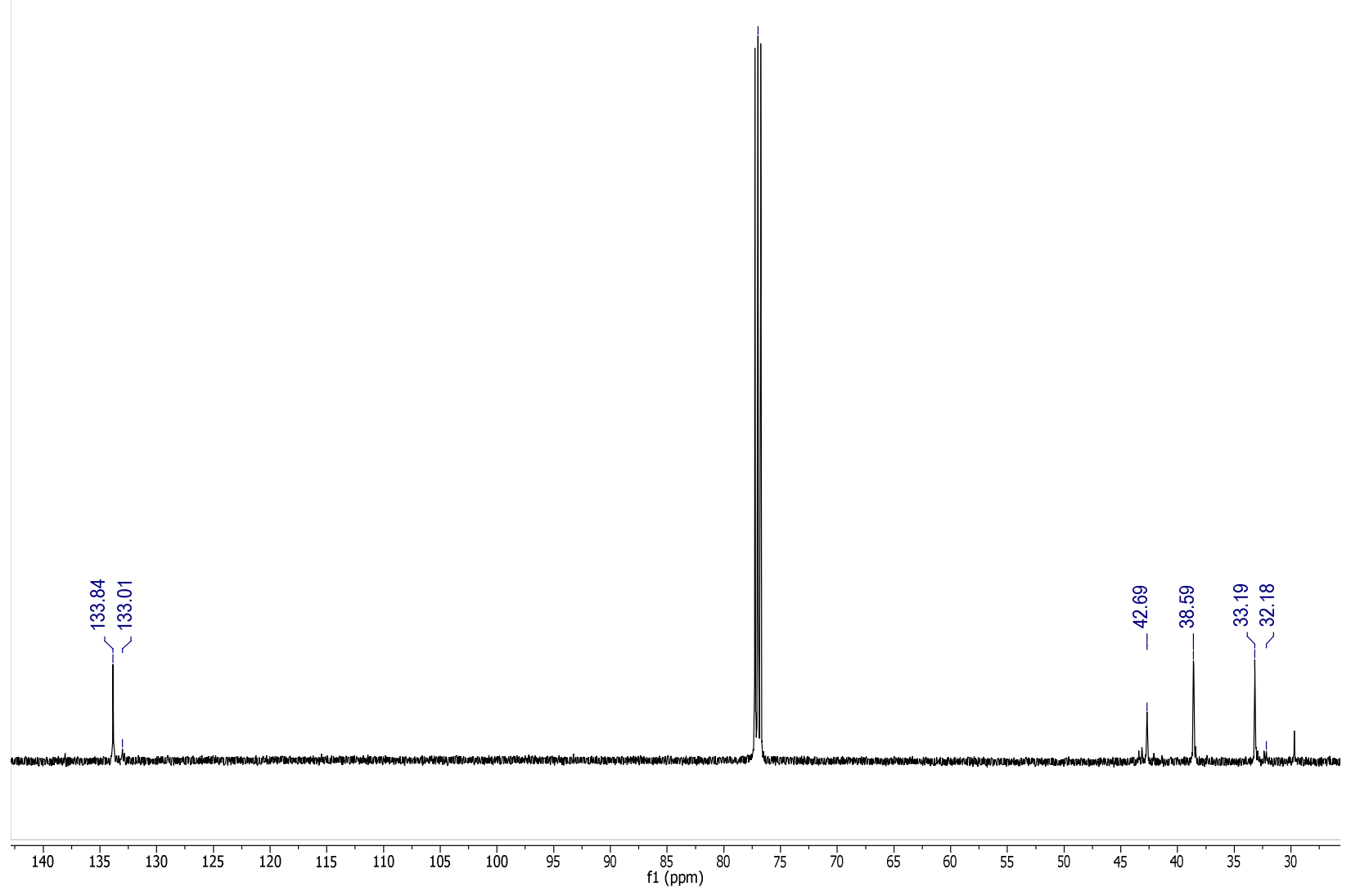

Figure S99. ${ }^{13} \mathrm{C}\left\{{ }^{1} \mathrm{H}\right\}$ NMR spectrum of $\sim 86 \%$ cis, syndiotactic enriched polynorbornene generated by $6\left(\mathrm{CDCl}_{3}, 125 \mathrm{MHz}\right)$ 


\section{X-ray crystallography of 7}

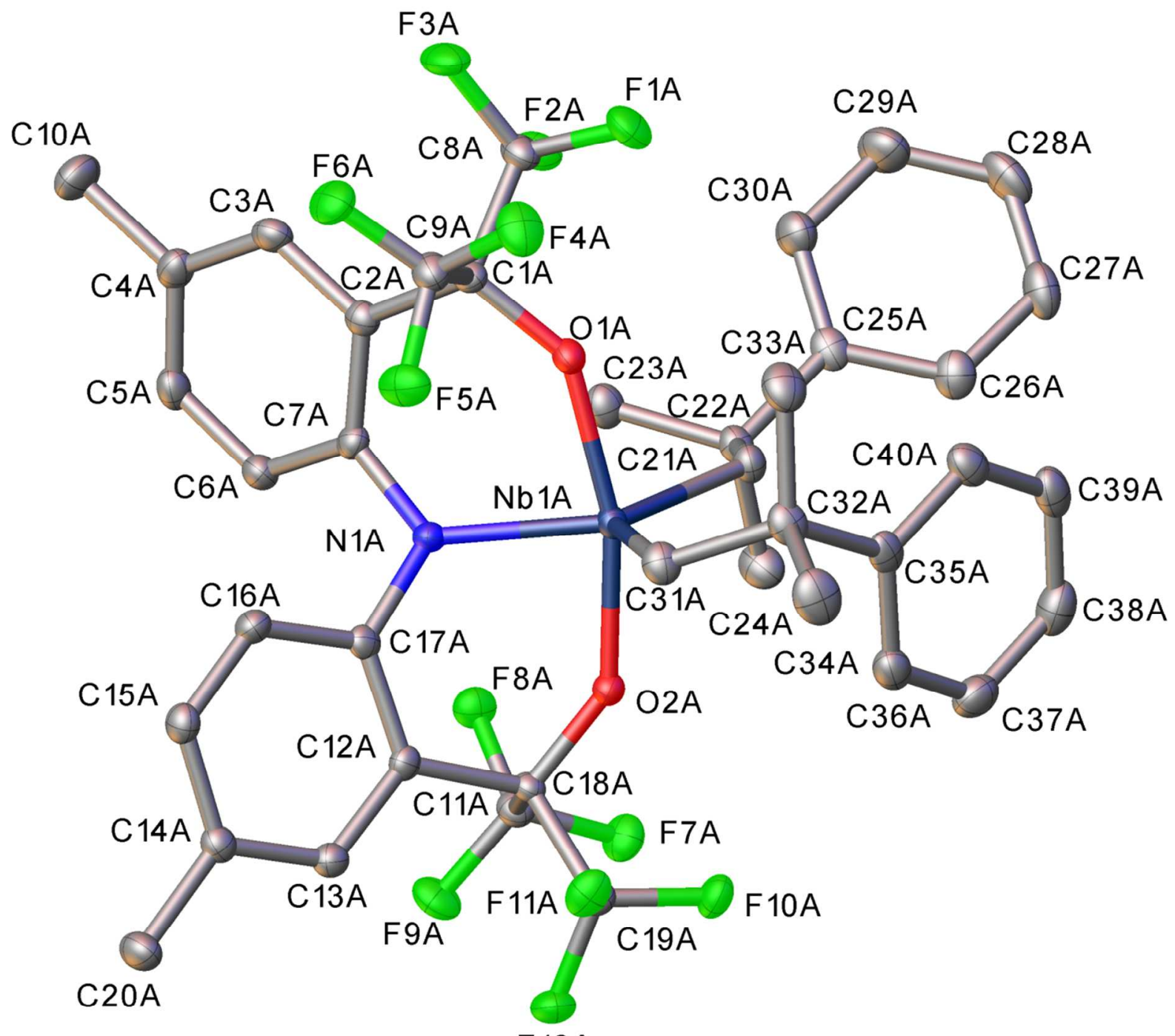

F12A

Figure S100. Molecular structure of 7 - only complex A shown (Complex 7B and all hydrogen atoms removed for clarity) 


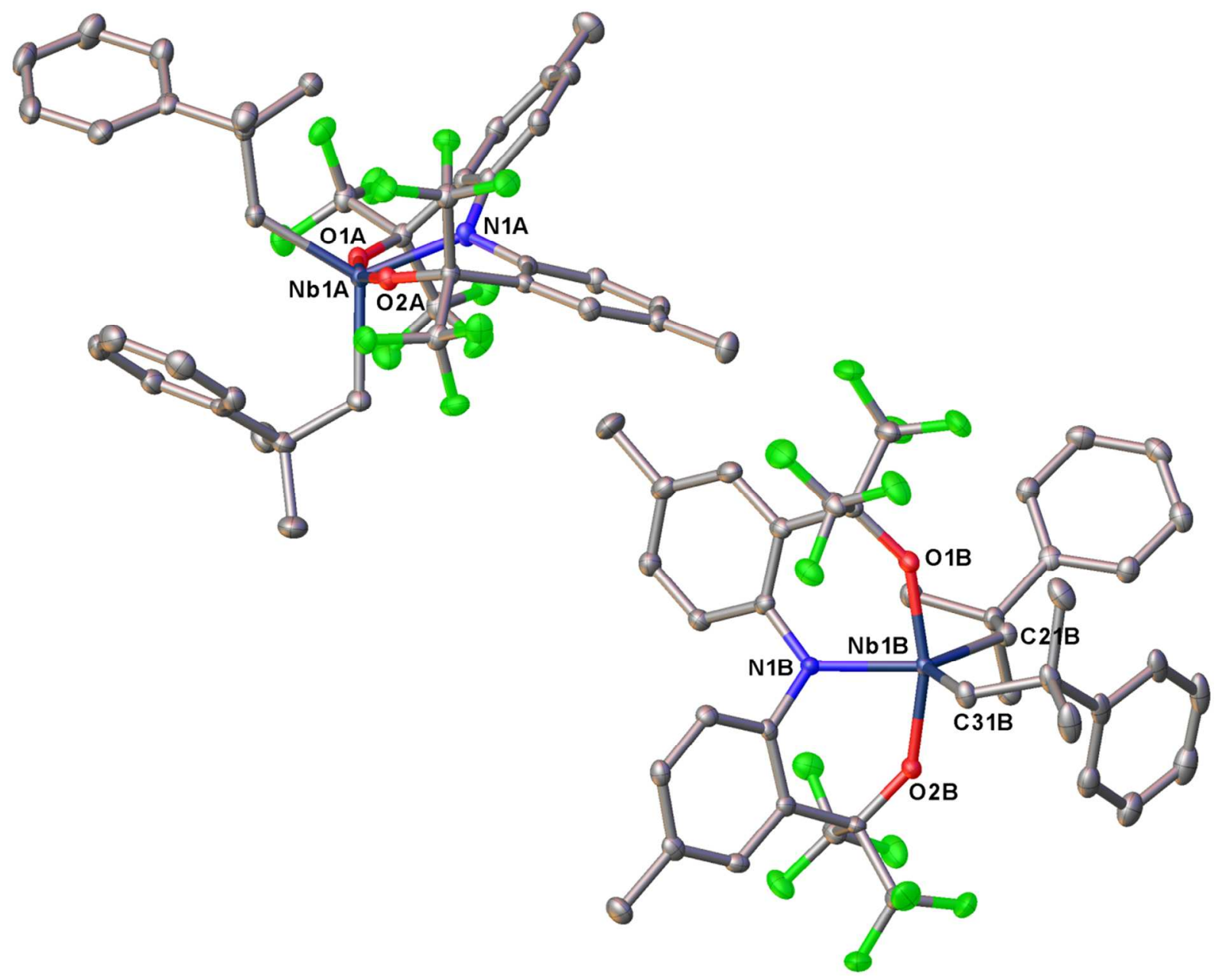

Figure S101. Asymmetric unit of 7 displaying the two chemically equivalent but crystallographically independent complexes (all hydrogen atoms removed for clarity).

$\underline{\text { X-Ray experimental: }}$ X-Ray Intensity data were collected at $100 \mathrm{~K}$ on a Bruker DUO diffractometer using Mo $\mathrm{K}_{\alpha}$ radiation $(\lambda=0.71073 \AA)$ and an APEXII CCD area detector. Raw data frames were read by program SAINT and integrated using 3D profiling algorithms. The resulting data were reduced to produce hkl reflections, their intensities, and estimated standard deviations. The data were corrected for Lorentz and polarization effects; numerical absorption corrections were applied based on indexed and measured faces. 
The structure was solved and refined in SHELXTL2014, ${ }^{7,8}$ using full-matrix least-squares refinement. The non- $\mathrm{H}$ atoms were refined with anisotropic thermal parameters and all of the $\mathrm{H}$ atoms were calculated in idealized positions and refined riding on their parent atoms. The asymmetric unit consists of two chemically equivalent but crystallographically independent complexes. In the final cycle of refinement, 17576 reflections (of which 12671 are observed with $\mathrm{I}>2 \sigma(\mathrm{I})$ ) were used to refine 1021 parameters and the resulting $\mathrm{R}_{1}, \mathrm{wR}_{2}$ and $\mathrm{S}$ (goodness of fit) were $3.48 \%, 7.03 \%$ and 0.947 , respectively. The refinement was carried out by minimizing the $\mathrm{wR}_{2}$ function using $\mathrm{F}^{2}$ rather than $\mathrm{F}$ values. $\mathrm{R}_{1}$ is calculated to provide a reference to the conventional $\mathrm{R}$ value but its function is not minimized. 
Table S3. Crystal data and structure refinement for 7

\begin{tabular}{|c|c|c|}
\hline Identification code & sud14 & \\
\hline Empirical formula & $\mathrm{C}_{40} \mathrm{H}_{38} \mathrm{NO}_{2} \mathrm{~F}_{12} \mathrm{Nb}$ & \\
\hline Formula weight & 885.62 & \\
\hline Temperature & $100.02 \mathrm{~K}$ & \\
\hline Wavelength & $\operatorname{Mo~K}_{\alpha}(\lambda=0.71073)$ & \\
\hline Crystal system & monoclinic & \\
\hline Space group & $P 2_{1} / \mathrm{c}$ & \\
\hline \multirow[t]{3}{*}{ Unit cell dimensions } & $\mathrm{a}=21.8594(9) \AA$ & $\alpha=90^{\circ}$ \\
\hline & $\mathrm{b}=19.7815(9) \AA$ & $\beta=108.6413(10)^{\circ}$ \\
\hline & $\mathrm{c}=18.6796(8) \AA$ & $\gamma=90^{\circ}$ \\
\hline Volume & $7653.5(6) \AA^{3}$ & \\
\hline Z & 8 & \\
\hline Density (calculated) & $1.537 \mathrm{~g} / \mathrm{cm}^{3}$ & \\
\hline Absorption coefficient $(\mu)$ & $0.409 \mathrm{~mm}^{-1}$ & \\
\hline $\mathrm{F}(000)$ & 3600 & \\
\hline Crystal size & $0.325 \times 0.224 \times 0.124 \mathrm{~mm}^{3}$ & \\
\hline Theta range for data collection & 1.423 to $27.498^{\circ}$ & \\
\hline Index ranges & $-27 \leq \mathrm{h} \leq 28,-25 \leq \mathrm{k} \leq 25,-24 \leq 1 \leq 22$ & \\
\hline Reflections collected & 125631 & \\
\hline Independent reflections & $17576\left[\mathrm{R}_{\text {int }}=0.0678, \mathrm{R}_{\text {sigma }}=0.0590\right]$ & \\
\hline Completeness to theta $=25.242^{\circ}$ & $100 \%$ & \\
\hline Absorption correction & Analytical & \\
\hline Max. and Min. transmission & 0.9667 and 0.9150 & \\
\hline Refinement method & Full-matrix least squares on $\mathrm{F}^{2}$ & \\
\hline Data / restraints / parameters & 17576 / 996 / 1021 & \\
\hline Goodness-of-fit on $\mathrm{F}^{2}$ & 0.947 & \\
\hline Final $\mathrm{R}$ indexes $[\mathrm{I}>2 \sigma(\mathrm{I})]$ & $\mathrm{R}_{1}=0.0348, \mathrm{wR}_{2}=0.0703[12671]$ & \\
\hline Final $\mathrm{R}$ indexes [all data] & $\mathrm{R}_{1}=0.0588, \mathrm{wR}_{2}=0.0777$ & \\
\hline Extinction coefficient & $\mathrm{n} / \mathrm{a}$ & \\
\hline Largest diff. peak and hole & 0.759 and -0.564 e $\AA^{-3}$ & \\
\hline
\end{tabular}

\footnotetext{
$\mathrm{R} 1=\Sigma\left(|| \mathrm{F}_{\mathrm{o}}|-| \mathrm{F}_{\mathrm{c}} \mid\right) / \Sigma\left|\mathrm{F}_{\mathrm{o}}\right| ; \mathrm{wR}_{1 / 2}=\left[\Sigma\left[\mathrm{w}\left(\mathrm{F}_{\mathrm{o}}^{2}-\mathrm{F}_{2}^{2}\right)^{2}\right] / \Sigma\left[\mathrm{w}\left(\mathrm{F}_{\mathrm{o}}^{2}\right)^{2}\right]\right]^{1 / 2}$

$\mathrm{S}=\left[\Sigma\left[\mathrm{w}\left(\mathrm{F}_{\mathrm{o}}{ }^{2}-\mathrm{F}_{\mathrm{c}}{ }^{2}\right)^{2}\right] /(\mathrm{n}-\mathrm{p})\right]^{1 / 2} ; \mathrm{w}=1 /\left[\sigma^{2}\left(\mathrm{~F}_{\mathrm{o}}{ }^{2}\right)+(\mathrm{m} * \mathrm{p})^{2}+\mathrm{n} * \mathrm{p}\right] ; \mathrm{p}=\left[\max \left(\mathrm{F}_{\mathrm{o}}{ }^{2}, 0\right)+2 * \mathrm{~F}_{\mathrm{c}}{ }^{2}\right] / 3 ; \mathrm{m}$ and $\mathrm{n}$ are constants.
} 


\section{References}

(1) O'Reilly, M. E.; Ghiviriga, I.; Abboud, K. A.; Veige, A. S. J. Am. Chem. Soc. 2012, 134, 11185 .

(2) VenkatRamani, S.; Pascualini, M. E.; Ghiviriga, I.; Abboud, K. A.; Veige, A. S. Polyhedron 2013, 64, 377.

(3) Fulmer, G. R.; Miller, A. J. M.; Sherden, N. H.; Gottlieb, H. E.; Nudelman, A.; Stoltz, B. M.; Bercaw, J. E.; Goldberg, K. I. Organometallics 2010, 29, 2176.

(4) Rosebrugh, L. E.; Marx, V. M.; Keitz, B. K.; Grubbs, R. H. J. Am. Chem. Soc. 2013, 135, 10032 .

(5) Autenrieth, B.; Schrock, R. R. Macromolecules 2015, 48, 2493.

(6) Hyvl, J.; Autenrieth, B.; Schrock, R. R. Macromolecules 2015, 48, 3148.

(7) Sheldrick, G. M. Acta Cryst. A. 2008, 64, 112.

(8) Sheldrick, G. M. Acta Cryst. C. 2015, 71, 3. 